Prepared for the U.S. Department of Energy

under Contract DE-AC05-76RL01830

\title{
Sampling Point Compliance Tests for 325 Building at Set-Back Flow Conditions
}

\author{
MY Ballinger \\ KP Recknagle \\ JA Glissmeyer \\ ST Yokuda \\ JM Barnett
}

May 2011

\section{Pacific Northwest}

NATIONAL LABORATORY

Proudly Operated by Battelle Since 1965 


\title{
DISCLAIMER
}

This report was prepared as an account of work sponsored by an agency of the United States Government. Neither the United States Government nor any agency thereof, nor Battelle Memorial Institute, nor any of their employees, makes any warranty, express or implied, or assumes any legal liability or responsibility for the accuracy, completeness, or usefulness of any information, apparatus, product, or process diselosed, or represents that its use would not infringe privately owned rights. Reference herein to any specific commercial product process, or service by trade name, trademark, manufacturer, or otherwise does not necessarily constitute or imply its endorsement, recommendation, or favoring by the United States Government or any agency thereof, or Battelle Memorial Institute. The views and opinions of authors expressed herein do not necessarily state or reflect those of the United States Government or any agency thereof.

\author{
PACIFIC NORTHWEST NATIONAL LABORATORY \\ operated by \\ BATTEI.I \\ for the \\ UNIILD STAILS DEPARIMLNT OI ENERGY \\ under Contract DE-AC05-76RLO1830 \\ Printed in the United States of America \\ Available to DOE and DOE contractors from the \\ Office of Scientific and Teclunical Information, \\ P.O. Box 62, Oak Ridge, TN 37831-0062; \\ ph: (865) $576-8401$ \\ fax: $(865) 576-5728$ \\ cmail: reports(a)adonis.osti.gov
}

\author{
Available to the public from the National Technical Information Service, \\ I I.S. Department of Commerce, 5285 Port Royal Rd., Springfield, VA 22161 \\ ph: (800) 553-6847 \\ fax: $(703) 605-6900$ \\ email: ordersàntis.fedworld.gov \\ online ordering: http://www.ntis.gov/ordering.htm
}

This document was printed on recyeled paper.

(9/2003) 


\title{
Sampling Point Compliance Tests for 325 Building at Set-Back Flow Conditions
}

\author{
MY Ballinger KP Recknagle \\ JA Glissmeyer ST Yokuda \\ JM Barnett
}

May 2011

Prepared for

the U.S. Department of Energy

under Contract DE-AC05-76RL01830

Pacific Northwest National Laboratory

Richland, Washington 99352 



\section{Summary}

The stack sampling system at the 325 Building (Radiochemical Processing Laboratory [RPL]) was constructed to comply with the American National Standards Institute's (ANSI's) Guide to Sampling Airborne Radioactive Materials in Nuclear Facilities (ANSI N13.1-1969). This standard provided prescriptive criteria for the location of radionuclide air-sampling systems. In 1999, the standard was revised (Sampling and Monitoring Releases of Airborne Radioactive Substances From the Stacks and Ducts of Nuclear Facilities [ANSI/Health Physics Society [HPS] 13.1-1999]) to provide performancebased criteria for the location of sampling systems.

Testing was conducted for the 325 Building stack to determine whether the sampling system would meet the updated criteria for uniform air velocity and contaminant concentration in the revised ANSI/HPS 13.1-1999 standard under normal operating conditions (Smith et al. 2010). Measurement results were within criteria for all tests. Additional testing and modeling was performed to determine whether the sampling system would meet criteria under set-back flow conditions. This included measurements taken from a scale model with one-third of the exhaust flow and computer modeling of the system with twothirds of the exhaust flow.

This report documents the results of the set-back flow condition measurements and modeling. Tests performed included flow angularity, uniformity of velocity, gas concentration, and particle concentration across the duct at the sampling location. Results are within ANSI/HPS 13.1-1999 criteria for all tests. These tests are applicable for the 325 Building stack under set-back exhaust flow operating conditions (980-45,400 cubic feet per minute [cfm]) with one fan running. The modeling results show that criteria are met for all tests using a two-fan configuration exhaust (flow modeled at 104,000 cfm). Combined with the results from the earlier normal operating conditions, the ANSI/HPS 13.1-1999 criteria for all tests are met for all configurations: one, two, or three fans (normal). 


\section{Acronyms and Abbreviations}

$\begin{array}{ll}\text { AD } & \text { aerodynamic diameter } \\ \text { ANSI } & \text { American National Standards Institute } \\ \text { CFD } & \text { computational fluid dynamics } \\ \text { CFR } & \text { Code of Federal Regulations } \\ \text { cfm } & \text { cubic feet per minute } \\ \text { COV } & \text { coefficient of variation } \\ \text { EPA } & \text { Environmental Protection Agency } \\ \text { ft } & \text { foot/feet } \\ \text { HPS } & \text { Health Physics Society } \\ \text { Hz } & \text { hertz } \\ \text { in } & \text { inch(es) } \\ \text { kg } & \text { kilogram(s) } \\ \text { L } & \text { liter(s) } \\ \text { mm } & \text { millimeter(s) } \\ \text { OPC } & \text { optical particle counter } \\ \text { PNNL } & \text { Pacific Northwest National Laboratory } \\ \text { RPL } & \text { Radiochemical Processing Laboratory (325 Building) }\end{array}$




\section{Contents}

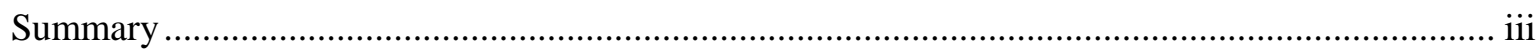

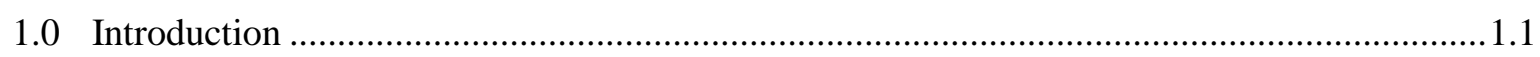

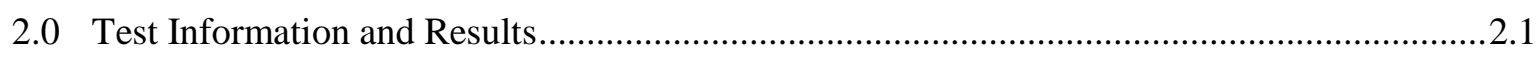

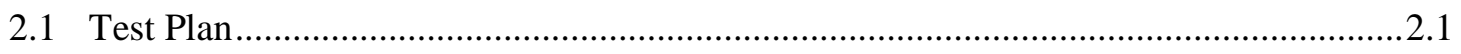

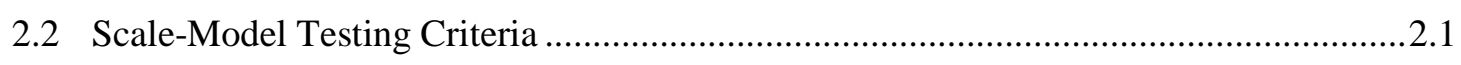

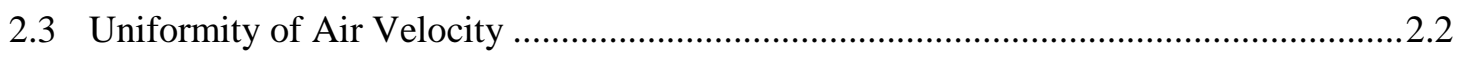

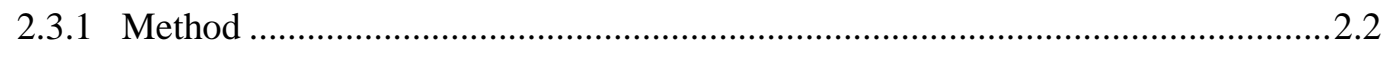

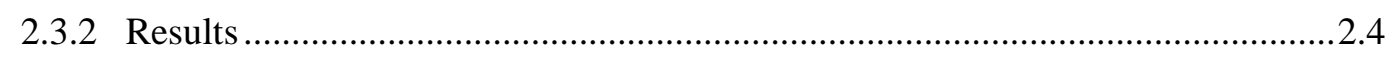

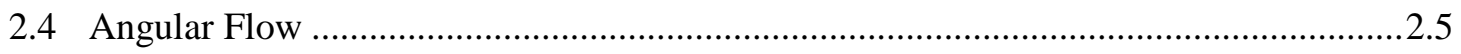

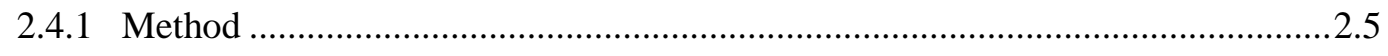

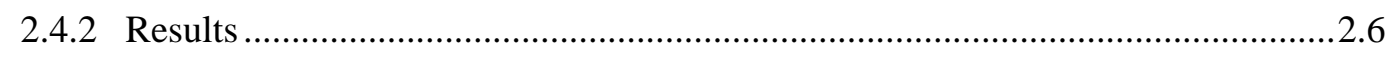

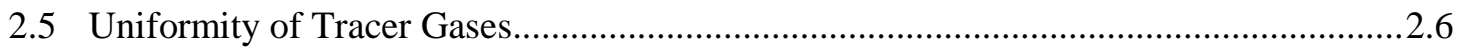

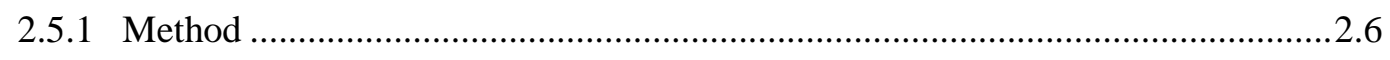

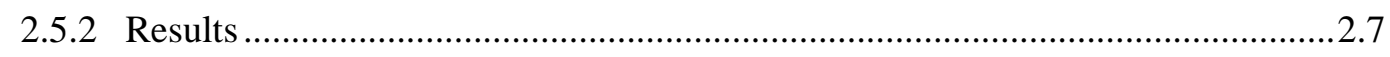

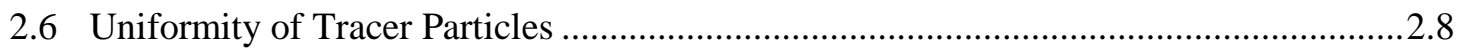

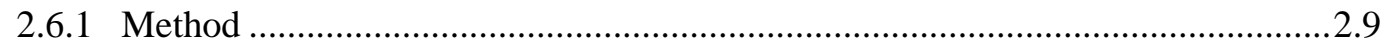

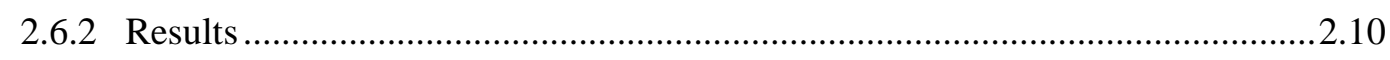

3.0 Computational Fluid Dynamics Model …........................................................................

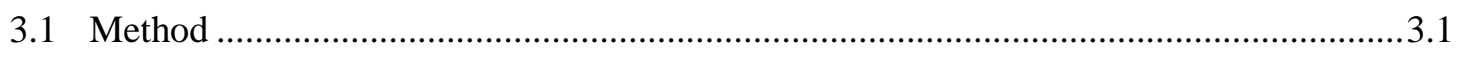

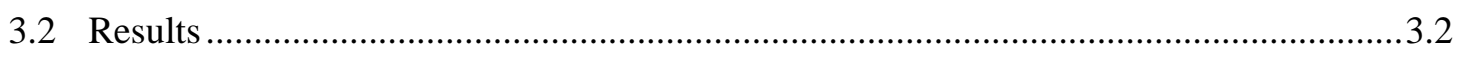

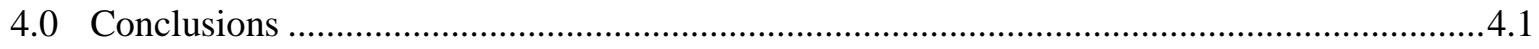

5.0 References .............................................................................................................. 5.1

Appendix A Test Plan for Qualifying the EP-325-01-S Stack Air Sampling Position for Set-Back

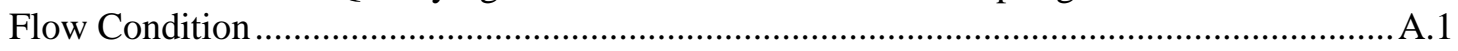

Appendix B Flow Calibration Procedure and Data.................................................................. B.1

Appendix C Velocity Uniformity Procedure and Data ................................................................. C.1

Appendix D Flow Angle Procedure and Data........................................................................... D. 1

Appendix E Tracer Gas Uniformity Procedure and Data .......................................................... E.1

Appendix F Particle Tracer Gas Uniformity Procedure and Data ..................................................1

Appendix G Calculations for Scale Model Criteria .......................................................................... 1

Appendix H Computational Fluid Dynamics Model Details .........................................................1 


\section{Figures}

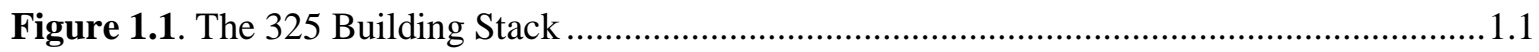

Figure 2.1. Side View of Scale Model of RPL Final Exhaust System.........................................2.2

Figure 2.2. Air Velocity as a Function of Fan Speed.................................................................... 2.3

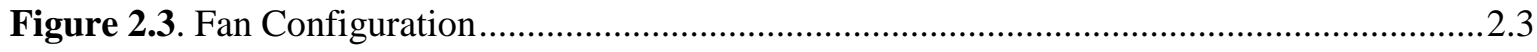

Figure 2.4. Velocity Uniformity Measuring Equipment ............................................................2.4

Figure 2.5. Velocity Measurements over the Measurement Grid for Run VT-1 …......................2.5

Figure 2.6. Type-S Pitot Tube and Protractor Level used to Measure Angular Flow.....................2.6

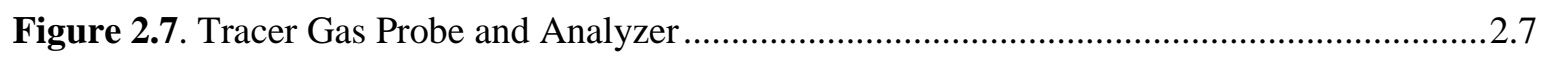

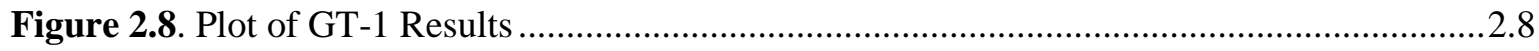

Figure 2.9. Typical Particle Generator Setup............................................................................2.9

Figure 2.10. Optical Particle Counter and Probe Arrangement for a Particle Uniformity Test....2.10

Figure 2.11. Bar Chart of PT-1 Results................................................................................. 2.11

Figure 3.1. Three-Dimensional Model Geometry of the RPL Final Effluent Stack ......................3.2

Figure 3.2. Flow Velocity Vectors in a Horizontal Plane ............................................................

Figure 3.3. Detail of Flow Vectors in a Horizontal Plane.............................................................. 3.3

\section{Tables}

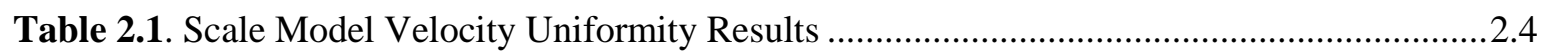

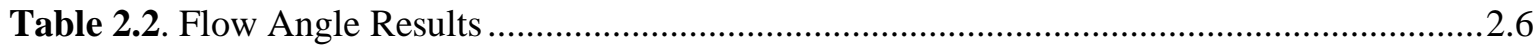

Table 2.3. Summary of Gas Tracer Uniformity Results During Simulated Setback Condition......2.8

Table 2.4. Particle Tracer Uniformity Results for the Center Two-Thirds of the Stack ..............2.10

Table 3.1. Results from CFD Modeling of RPL Final Exhaust System........................................3.3

Table 4.1. Summary of Test Results for Low Flow RPL Exhaust ................................................. 


\subsection{Introduction}

The stack sampling system at the 325 Building (Radiochemical Processing Laboratory [RPL]) was constructed to comply with the American National Standards Institute's (ANSI's) Guide to Sampling Airborne Radioactive Materials in Nuclear Facilities (ANSI N13.1-1969). This standard provided prescriptive criteria for the location of radionuclide air-sampling systems. In 1999, the standard was revised (Sampling and Monitoring Releases of Airborne Radioactive Substances From the Stacks and Ducts of Nuclear Facilities [ANSI/Health Physics Society \{HPS \} 13.1-1999]) to provide performancebased criteria for the location of sampling systems. Testing was conducted for the 325 Building stack to determine whether the sampling system would meet the updated criteria for uniform air velocity and contaminant concentration in the revised ANSI/HPS 13.1-1999 standard.

The 325 Facility emission point exhausts air from all areas of the building where radioactive materials are handled. The exhaust stream passes through high-efficiency particulate air filters located just upstream of the exhaust fans. The stack (Figure 1.1) is 88 feet (ft) tall and $8 \mathrm{ft}$ in diameter, with flows at approximately $140,000 \mathrm{cfm}$. The sampling system is located approximately $80 \mathrm{ft}$ above the ground (see the platform in Figure 1.1).

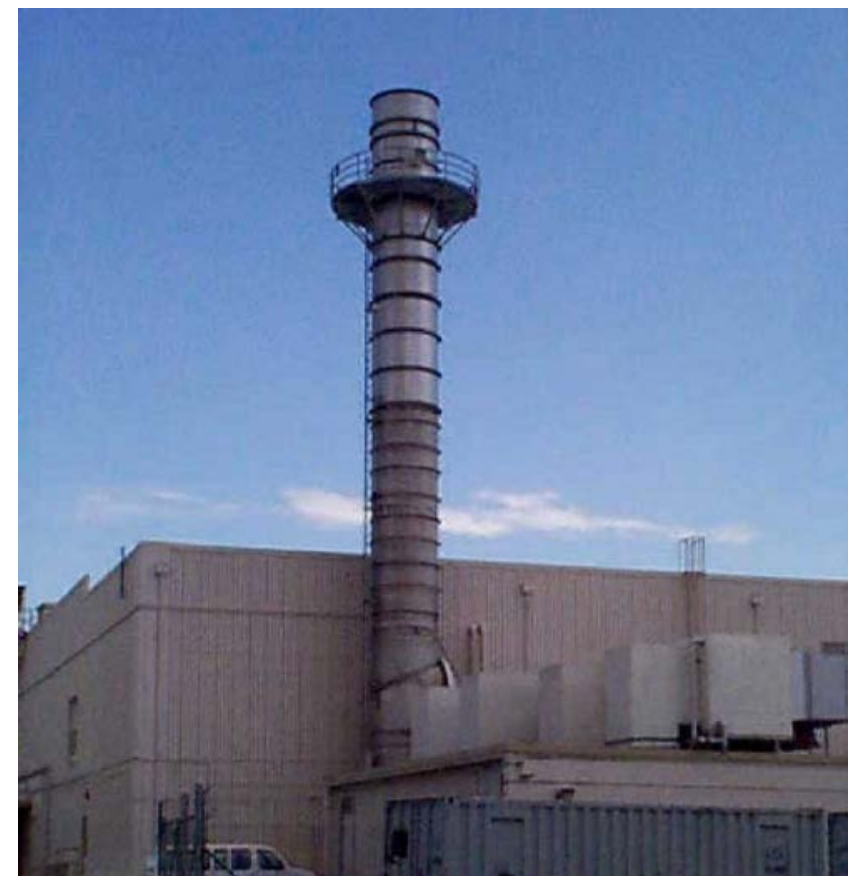

Figure 1.1. The 325 Building Stack

Because of the difficulty in taking measurements at the elevated sampling location and to avoid possible disruptions to facility operations, a scale model was used for the tests. The scale model was fabricated on an outside concrete pad. Scaffolding was used as support for the stack section and to gain access to the sample ports. Four variable speed fans were connected to the scale model in a configuration geometrically similar to the actual stack. The 325 facility normally operates only three of the four fans and alternates the standby fan. Therefore, a similar arrangement was used in the first set of testing (Smith et al. 2010). Testing results from measurements taken in 2002 demonstrated that the sampling 
location on the 325 Building met the criteria in the 1999 standard for a well mixed location under normal operating conditions.

Subsequent testing was conducted in 2004 to determine whether the stack sampling system at the 325 Building would meet the 1999 standard criteria for uniform air velocity and contaminant concentration if the exhaust air flow was reduced to one-third to reduce energy use. The same scale model was used for these reduced flow tests. Tests performed included flow angularity, uniformity of velocity, gas concentration, and particle concentration across the duct at the sampling location. Tests were conducted with the fan nearest to the stack operating and the fan farthest from the stack operating in order to test the two extremes of fan configuration. Results are within ANSI/HPS 13.1-1999 criteria for all tests. These tests are applicable for the 325 Building stack under reduced flow operating conditions (below 45,400 cfm) with any single fan running.

In addition to the scale model testing, a computational fluid dynamics (CFD) model was used to predict results for a two-fan configuration. Results from the fluid dynamics model were also within ANSI/HPS 13.1-1999 criteria for all tests and bridged the data between the one- and three-fan configurations tested using the scale model. 


\subsection{Test Information and Results}

This section discusses testing of a scale model of the RPL final exhaust system to determine whether the sampling system location met the criteria in ANSI/HPS N13.1-1999 for a well mixed location. The approach, test methods, and results are provided.

\subsection{Test Plan}

The objective of these tests was to demonstrate whether the EP-325-01-S exhaust stack meets the applicable regulatory criteria regarding the placement of the air-sampling probe under reduced exhaust flow. This has already been demonstrated for the normal flow-rate with three operating fans (Ballinger et al. 2004, Smith et al. 2010). This retest demonstrates whether the criteria are still met if the system flow-rate is reduced from the normal value by a factor of three when only one fan is used. The tests were conducted by Pacific Northwest National Laboratory (PNNL) staff. The standard governing the performance of the tests, test methods, and acceptance criteria is ANSI/HPS N13.1-1999, Sampling and Monitoring Releases of Airborne Radioactive Substances From the Stack and Ducts of Nuclear Facilities (ANSI 1999). The test plan for this series is included in Appendix A.

\subsection{Scale-Model Testing Criteria}

The ANSI/HPS N13.1-1999 standard contains acceptance criteria for the use of a similarly designed stack, including a scale-model, as a substitute for the actual stack (Section 5.2.2.2 of ANSI/HPS N13.11999). The acceptance criteria are summarized as follows:

1. The scale model and its sampling location must be geometrically similar to the actual stack, with components influencing contaminant mixing and velocity profile proportional in the scale model proportional to those in the actual stack.

2. The scale model's mean velocity times hydraulic diameter must be within a factor of six of the actual stack. The stack diameter of the scale model must be at least 250 millimeters ( $\mathrm{mm}$ ) at the sampling location. The Reynolds number for the prototype and scale model stacks must be greater than 10,000.

The scale model results are considered valid if:

1. The velocity profile in the actual stack meets the uniformity criteria, and

2. The difference between velocity coefficients of variation (COVs) of the two systems is not more than $5 \%$ COV units.

3. The sampling location is placed at a geometrically similar location in the actual and scale model stacks.

A scale model was used for these tests because of the difficulty in taking measurements at the elevated sampling location and to avoid possible disruptions to operations in this nuclear facility. The scale model of the RPL final exhaust system was designed with consideration for the above criteria. The portion of the RPL exhaust system containing the final exhaust fans, downstream ducting, and stack was considered a sufficient segment to model velocity and contaminant mixing adequately. Several scales were considered that fit the criteria above, with a 1:5.33 scale selected based on convenient stack and duct size (the scale model was $18 \mathrm{ft}$ high and 18 inches [in; $46 \mathrm{~cm}$ ] in diameter), and similarity of stack 
velocity. See the test plan in Appendix A for more detail on considerations for the scale model. Figure 2.1 shows a side view of the scale model after it was completed.

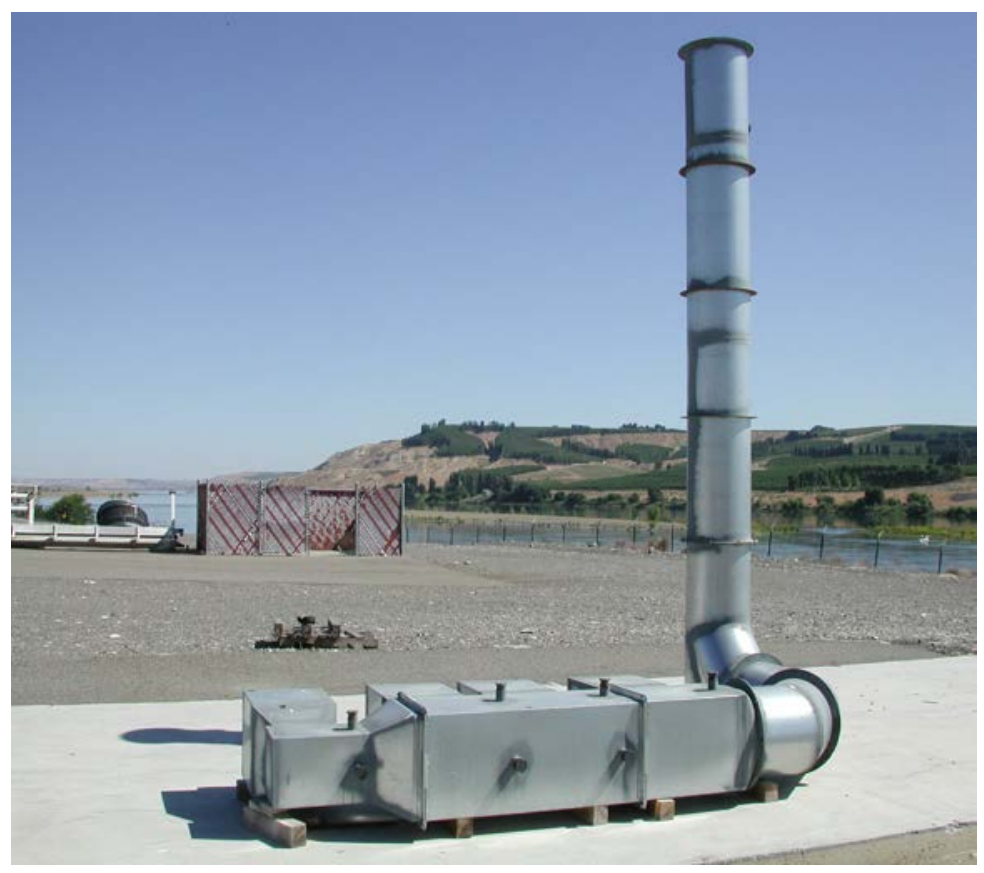

Figure 2.1. Side View of Scale Model of RPL Final Exhaust System

\subsection{Uniformity of Air Velocity}

The uniformity of air velocity in the stack cross section where the air sample is being extracted ensures that the air momentum in the stack is well mixed. The method used to demonstrate air velocity uniformity and the results obtained are detailed in the following sections.

\subsubsection{Method}

To facilitate the performance of this and subsequent tests, it was first necessary to correlate the fan speed controller (a variable frequency drive) with the stack flowrate. Following the procedure in Appendix B, a velocity uniformity measurement (Run VT-LOW1) was made at the midrange fan speed setting (30 hertz [Hz]) to identify a single measurement point that best represented the average velocity. The air velocity was then measured at that point as a function of fan speed setting. The results are plotted in Figure 2.2. The set point for the balance of the tests reported here $(37.1 \mathrm{~Hz})$ was estimated from the plot. The Run VT-LOW1 also provided a data point for velocity uniformity. 


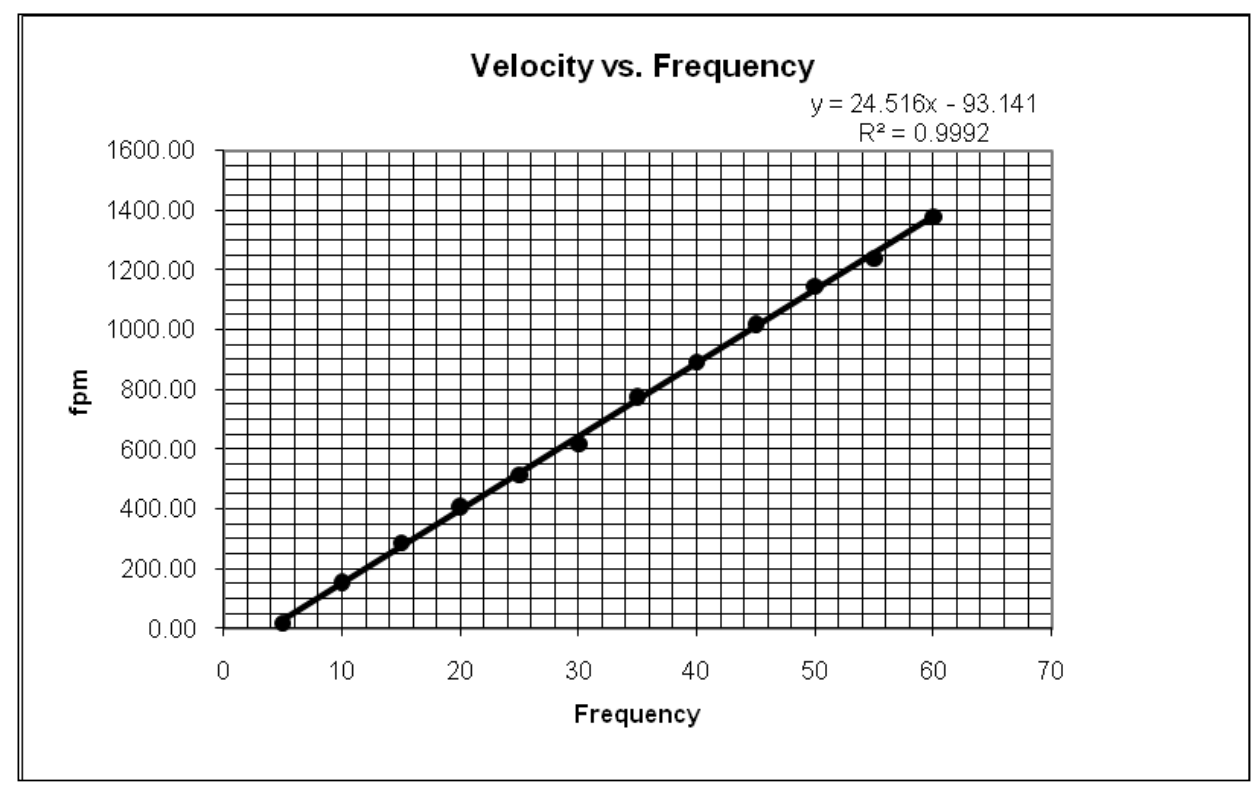

Figure 2.2. Air Velocity as a Function of Fan Speed

For this and most other tests, either the fan nearest to or farthest from the stack (Figure 2.3) was running. These configurations were used because they are expected to bracket the cases for stack mixing. Disturbances closer to the sampling port are expected to be more disruptive to uniform mixing than those further away; thus, the near fan configuration should provide the worst case for velocity uniformity.

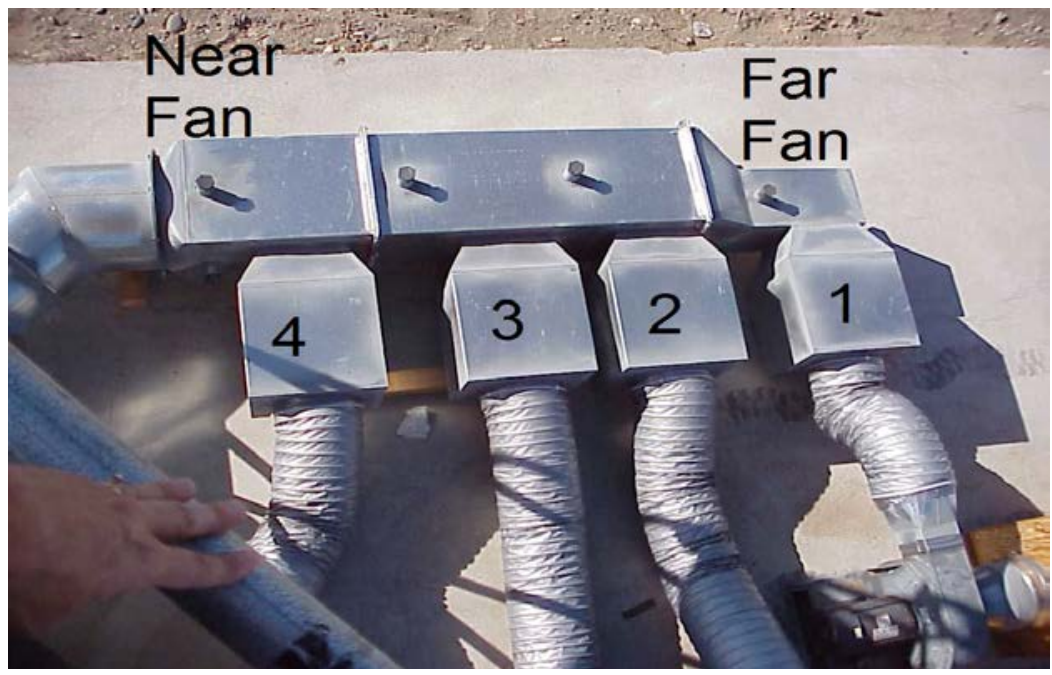

Figure 2.3. Fan Configuration

The method to determine velocity uniformity is an adaptation of 40 Code of Federal Regulations (CFR) 60, Appendix A, Method 1. The equipment included a standard Prandtl-type pitot tube and a calibrated electronic manometer as shown in Figure 2.4. The procedure is detailed in Appendix C. The grid of measurement points was laid out in accordance with the U.S. Environmental Protection Agency (EPA) procedure for eight points on each of two linear traverses, arranged perpendicular to each other. 
The center point was added for additional information over what is otherwise a long distance between points 4 and 5 . Thus, there were 9 points along the northeast/southwest direction and also along the southeast/northwest direction.

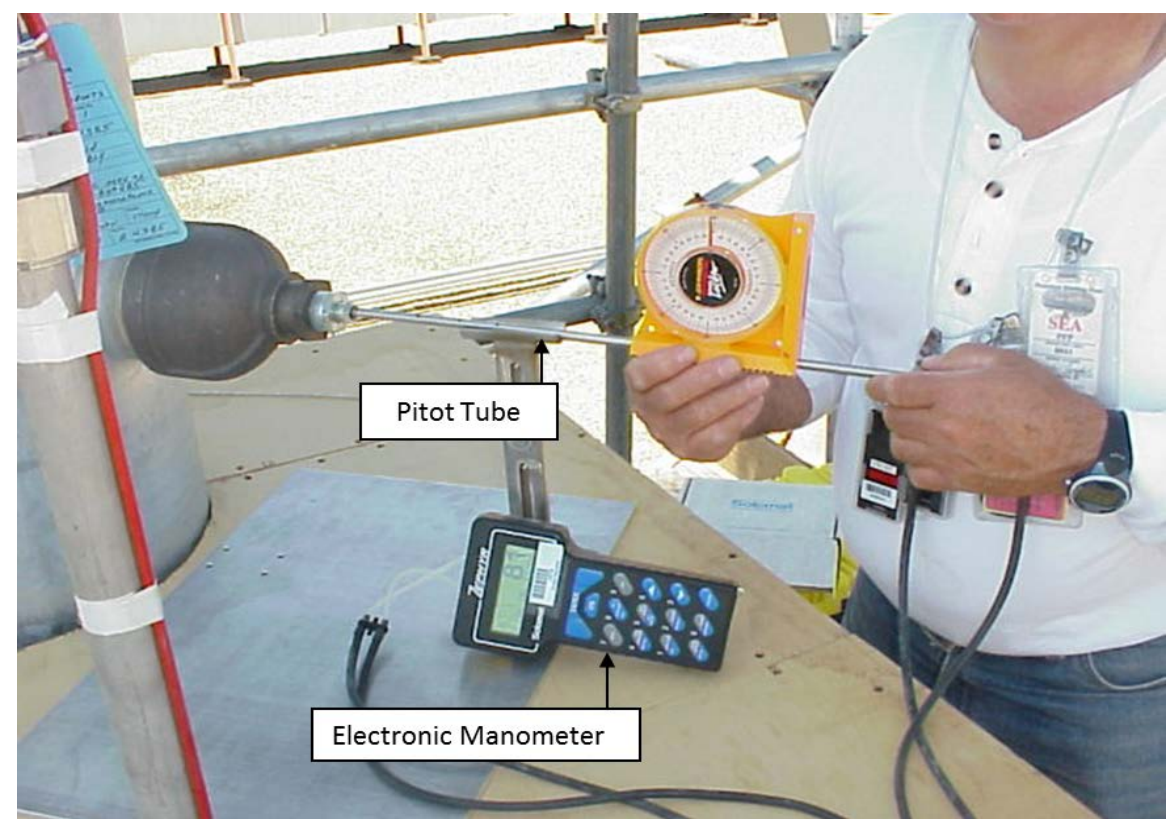

Figure 2.4. Velocity Uniformity Measuring Equipment

\subsubsection{Results}

The acceptance criterion for uniformity of air velocity is that the COV of the air velocity must be $\leq 20 \%$ across the center two-thirds of the area of the stack. The measured COVs for air velocity in the center two-thirds of the area of the scale model stack are listed in Table 2.1 and range from 1.6 to 9.4. The data sheets are included in Appendix C. All of the scale model test results for velocity uniformity meet the criterion that the air velocity COVs be $\leq 20 \%$. On the actual stack with one fan operating, the full-scale velocity uniformity COVs range from 3.9 to 10.7 (Recknagle et al. 2008). The scale model and the full scale results show good agreement. This agreement meets the acceptance criterion ( $\pm 5 \% \mathrm{COV}$ units) for validating the scale model results. Figure 2.5 shows a bar graph of the mean velocity measured at each point for Run VT-1, one of the scale model results.

Table 2.1. Scale Model Velocity Uniformity Results

\begin{tabular}{lccc}
\hline \multicolumn{1}{c}{ Runs } & $\begin{array}{c}\text { Fan Frequency } \\
\text { Setting (Hz) }\end{array}$ & $\begin{array}{c}\text { Stack Flow Rate } \\
\text { (cfm) }\end{array}$ & \% COV \\
\hline \multicolumn{4}{c}{ Near Fan } \\
VT-LOW1 & 30 & 1100 & 9.4 \\
VT-1 & 37.1 & 1389 & 4.0 \\
VT-3 & 37.1 & 1421 & 3.4 \\
\multicolumn{2}{c}{ Far Fan } \\
VT-2 & 37.1 & 1334 & 1.6 \\
VT-4 & 37.1 & 1335 & 1.9 \\
\hline
\end{tabular}




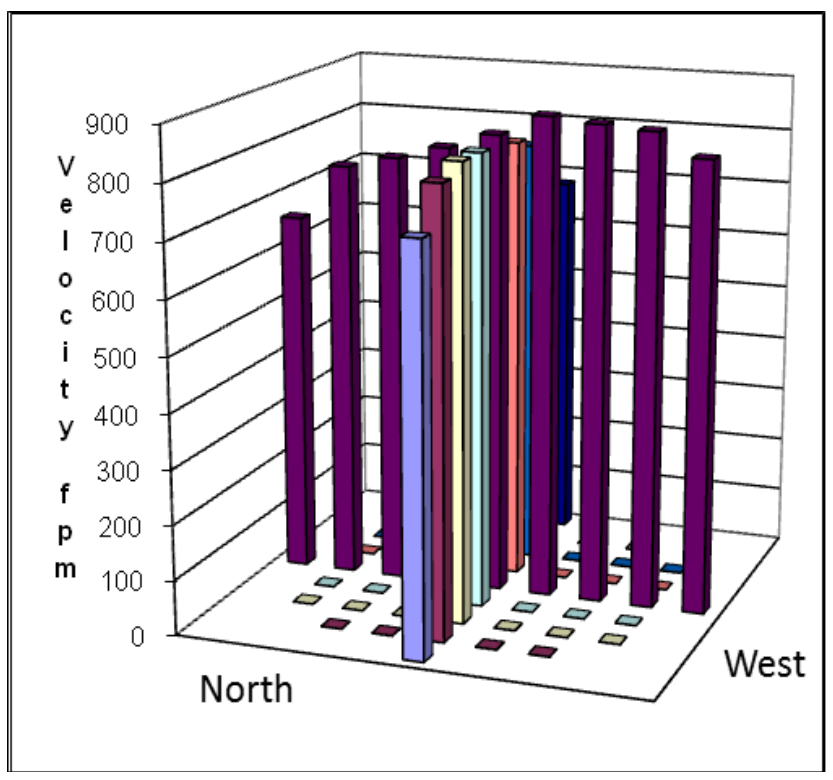

Figure 2.5. Velocity Measurements over the Measurement Grid for Run VT-1

\subsection{Angular Flow}

The angular flow measurement in the stack cross section where the air sample is being extracted ensures that the flow angle is not more than $20^{\circ}$ across the sampling plane. The method used to demonstrate the angular flow and the results obtained are presented below.

\subsubsection{Method}

The test method used was based on 40 CFR 60, Appendix A, Method 1, Section 2.4, "Verification of the Absence of Cyclonic Flow." This test was conducted at the scaled set-back flowrate in the model stack. Measurements were made using a type-S pitot tube, a slant tube or electronic manometer, and a protractor level attached to the pitot tube (Figure 2.6). The flow angle was measured at the elevation of the sampling nozzle and at the same points as those used for the velocity uniformity test. The pitot tube was rotated until a null differential pressure reading was obtained, and the angle of rotation was then recorded. Appendix D provides the detailed procedure. 


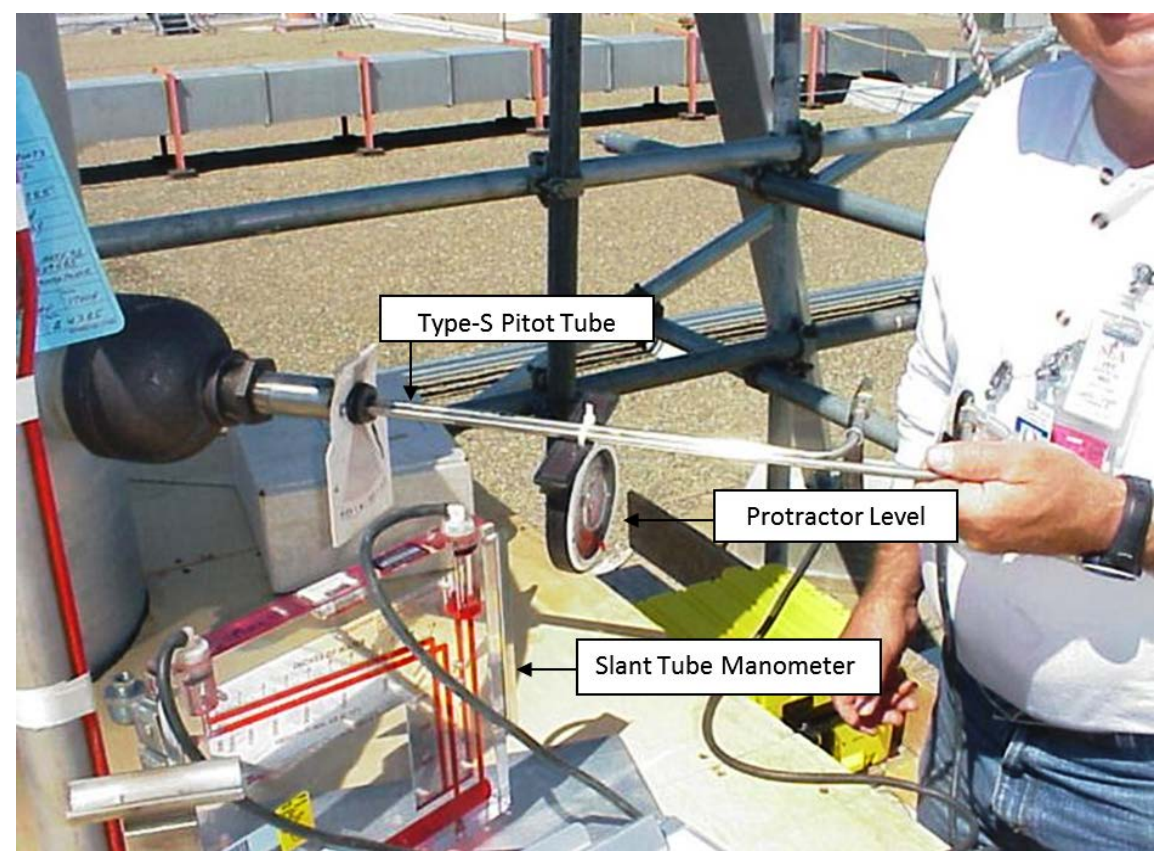

Figure 2.6. Type-S Pitot Tube and Protractor Level used to Measure Angular Flow

\subsubsection{Results}

The acceptance criterion for angular flow is an average flow-angle of $<20^{\circ}$ across the sampling plane. Measurements were made at the same grid points as for the velocity uniformity. The acceptance criterion $\left(\leq 20^{\circ}\right)$ was met in all cases. The results range from 8.0 to $14.3^{\circ}$. Table 2.2 shows a summary of the angular flow testing results. The data sheets for the angular flow test are presented in Appendix D.

Table 2.2. Flow Angle Results

\begin{tabular}{lcc}
\hline Runs & $\begin{array}{c}\text { Fan Frequency } \\
\text { Setting, Hz }\end{array}$ & $\begin{array}{c}\text { Mean Flow } \\
\text { Angle }\end{array}$ \\
\hline FA-1 & Far Fan & \\
& 37.1 & 9.5 \\
FA-2 & Near Fan & \\
FA-3 & 37.1 & 14.3 \\
\hline
\end{tabular}

\subsection{Uniformity of Tracer Gases}

A uniform gas contaminant concentration at the sampling plane enables the extraction of samples that represent the true gas concentration within the stack. Testing for uniformity of tracer gases at the sampling plane was conducted on the scale model stack at the scaled set-back flowrate.

\subsubsection{Method}

The concentration uniformity is demonstrated with a tracer gas (sulfur hexafluoride) injected into the exhaust duct, in the same area as the discharge from the model heat recovery boxes for the near- and far- 
fans. The concentration of the tracer gas is then measured at the sampling location using the same grid of points as used in the other tests. From the measurements, the COV and maximum deviation from the mean are calculated as measures of uniformity.

The gas samples are withdrawn from the stack through a simple probe and a gas analyzer (Figure 2.7). A Bruel and Kjaer (Naerum, Denmark) Model 1302 calibrated for the tracer gas, is used for the measurements. The procedure and data sheets are detailed in Appendix E.

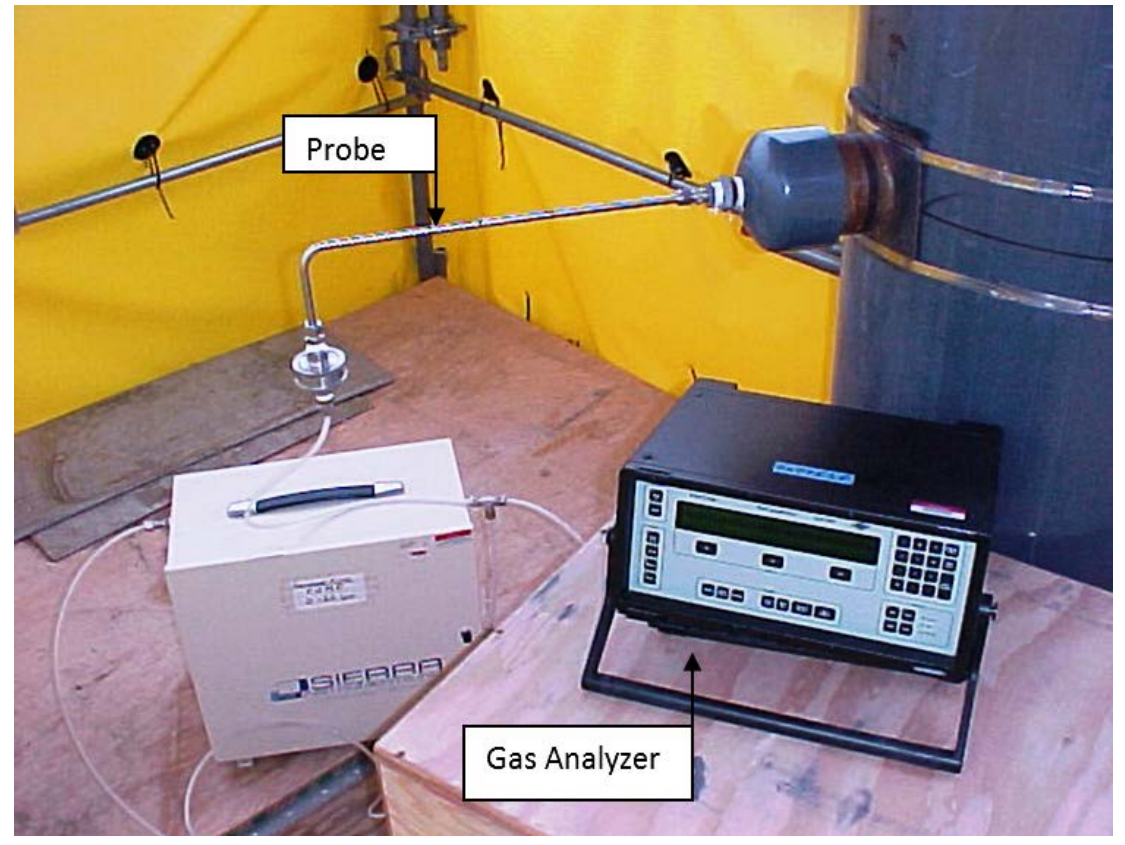

Figure 2.7. Tracer Gas Probe and Analyzer

\subsubsection{Results}

The acceptance criteria for uniformity of tracer gases are:

1) the COV of the tracer gas concentration be $\leq 20 \%$ across the center two-thirds of the sampling plane

2) the average concentration, for each measurement point, differ from the mean concentration by $<30 \%$.

Table 2.3 lists the tests performed and their results. Five injection points were used at each injection location. Corner injections were made within 1 in (25\% of a hydraulic diameter) of the walls at the corners of the duct (Figure 2.3). The worst case result was repeated as Run GT-11, where the uniformity results ranged from 1.6 to $5.5 \% \mathrm{COV}$. The absolute value of the maximum deviations from the means ranged from 2.6 to $12.6 \%$. In all cases, the acceptance criteria were met. Figure 2.8 is a bar graph of the results of Run GT-1. 
Table 2.3. Summary of Gas Tracer Uniformity Results During Simulated Setback Condition

\begin{tabular}{llrr}
\hline \multicolumn{1}{c}{ Run } & Injection Point & \% COV & $\begin{array}{c}\text { Max \% } \\
\text { Dev }\end{array}$ \\
\hline GT-1 & Near Fan & \\
GT-11 & Top-south & 4.75 & 12.6 \\
GT-2 & Top-south & 5.48 & -10.2 \\
GT-3 & Top-north & 2.49 & -4.2 \\
GT-4 & Center & 3.54 & 6.3 \\
GT-5 & Bottom south & 3.75 & -6.9 \\
\hline \multicolumn{4}{r}{$\quad$ Far Fan } \\
GT-6 & Bottom-north & 3.88 & 8.1 \\
GT-7 & Top-south & 1.63 & -2.6 \\
GT-8 & Top-north & 1.82 & 3.4 \\
GT-10 & Center & 2.28 & 4.8 \\
GT-9 & Bottom south & 2.22 & 4.1 \\
\hline
\end{tabular}

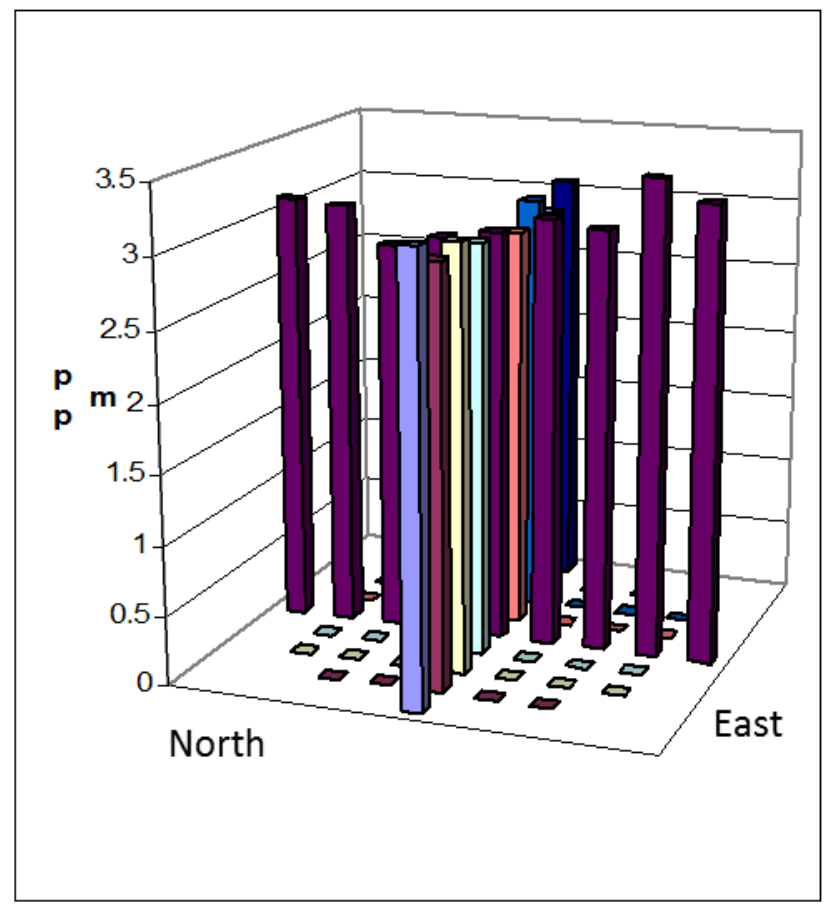

Figure 2.8. Plot of GT-1 Results

\subsection{Uniformity of Tracer Particles}

A uniform particulate contaminant concentration at the sampling plane enables the extraction of samples that represent the true particulate concentration within the stack. Testing for uniformity of tracer particles at the sampling plane was conducted on the scale model stack. The method for determining uniformity of tracer particles and the results of the tests are detailed in the following sections. 


\subsubsection{Method}

The test method for uniformity of tracer particles is similar to that of tracer gases, with the tracer gas replaced by tracer particles. However, only the centerline injection position is required. The concentration of the tracer particles, in the size range of interest, was measured at the same test points used in the other tests. Spraying vacuum-pump oil through a nozzle mounted inside a chamber produced the particles measured by the testing. These particles were then injected into the duct entrained in a stream of compressed air as shown in Figure 2.9.

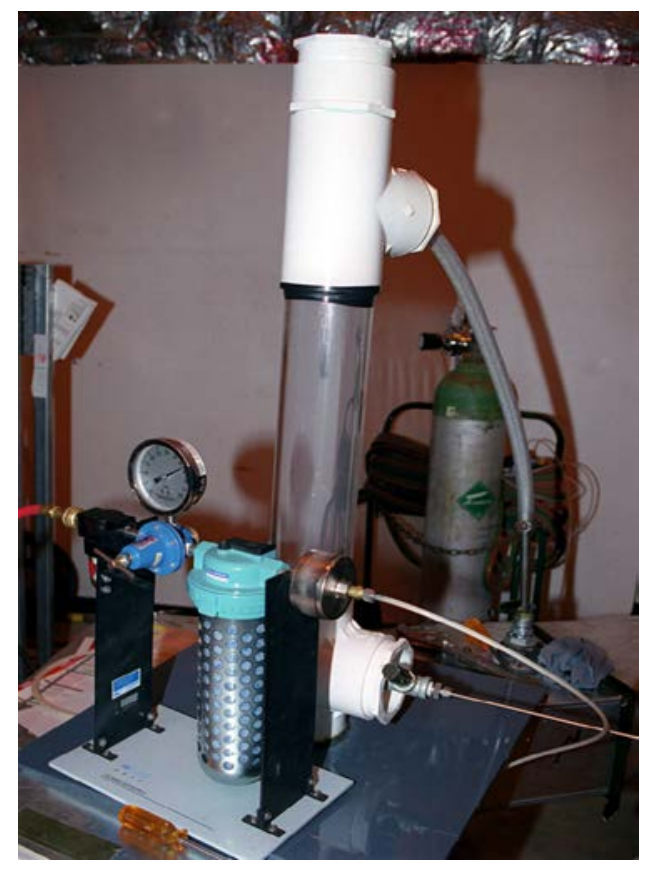

Figure 2.9. Typical Particle Generator Setup

A simple probe was used to extract the sample from the stack and transport it to the optical particle counter ${ }^{1}$ (OPC) arranged as shown in Figure 2.10. The OPC sorts the number of particles into six size channels. Only the readings from the size channel that measures particles in the 9 to $11 \mu \mathrm{m}$ size range are used for statistical calculations. Each data point consists of the number of particles counted during a oneminute sampling period. Three readings were taken at each point and averaged. The COV of the average concentration readings at each point is calculated and the result compared to the acceptance criterion for uniformity. The detailed procedure and data sheets are included in Appendix F.

\footnotetext{
${ }^{1}$ Met-One Model A2408, Hach Analytics, Grants Pass, OR.
} 


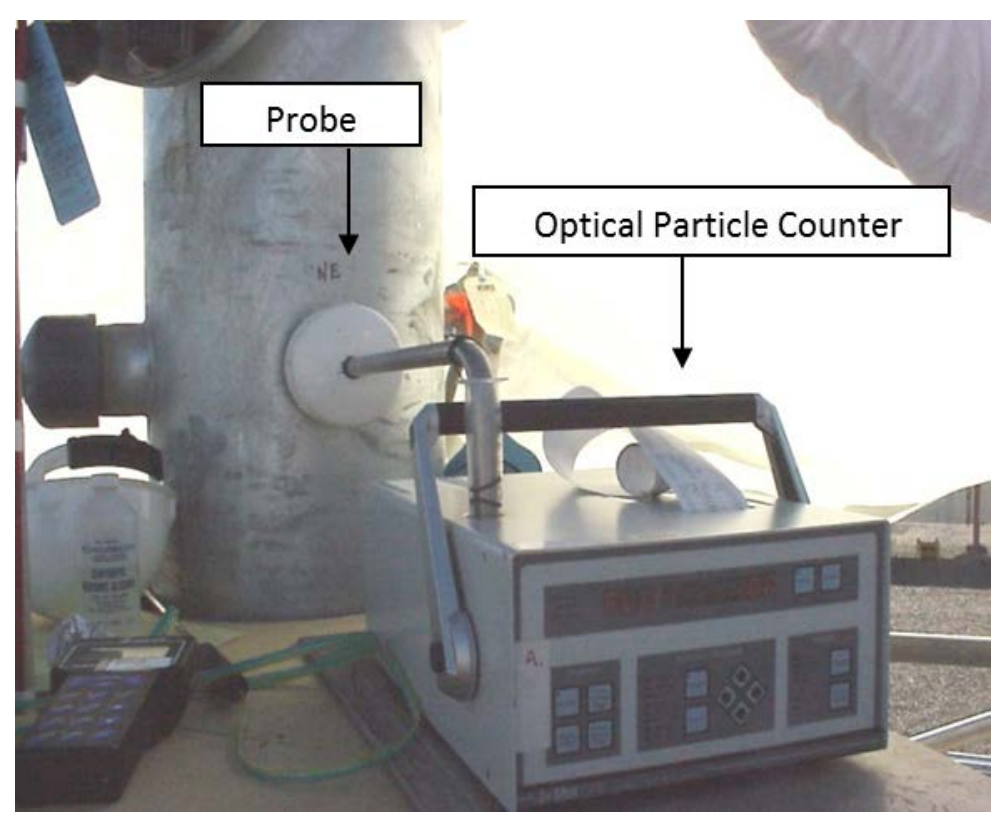

Figure 2.10. Optical Particle Counter and Probe Arrangement for a Particle Uniformity Test

\subsubsection{Results}

The acceptance criterion for uniformity of tracer particle is a COV less than $20 \%$ for tracer particles of the $10-\mu \mathrm{m}$ range across the center two-thirds of the sampling plane.

The particle concentration uniformity is demonstrated with tracer particles injected into the exhaust duct, in the same area as the discharge from the model heat recovery boxes for the near- and far-fans. Tests were conducted at the simulated set-back flowrate. The results are summarized in Table 2.4 and the data sheets are included in Appendix F. The results show slightly more uniformity for the near fan configuration than for the far fan configuration. However, in all cases, the performance criterion was met. Figure 2.11 is a bar chart showing the normalized concentration data for the worst case test, PT-1.

Table 2.4. Particle Tracer Uniformity Results for the Center Two-Thirds of the Stack

\begin{tabular}{llcc}
\hline & Injection Point & $\begin{array}{c}\text { Un-normalized } \\
\text { \% COV }\end{array}$ & $\begin{array}{c}\text { Normalized } \\
\text { \% COV }\end{array}$ \\
\hline & & Far Fan & \\
PT-1 & Center & 10.5 & 11.6 \\
PT-3 & Center & 10.5 & 7.4 \\
PT-2 & Center & Near Fan & 8.3 \\
\hline
\end{tabular}




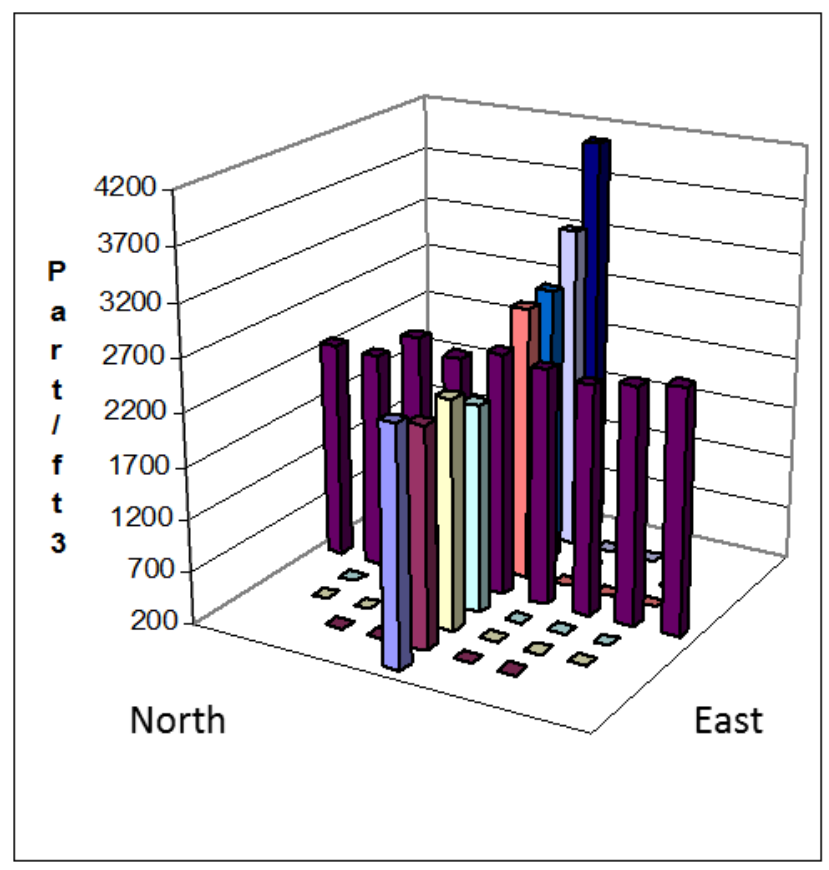

Figure 2.11. Bar Chart of PT-1 Results

The COV results are shown in Table 2.4 with and without any normalization with time. The results after normalization also are shown. The normalization method adjusts all of the concentration readings by the same amount so that the center point readings taken from the two traverse directions were equalized. The effect of normalization would be more pronounced in cases where there was a shift in concentration with time. All of the normalized data met compliance criteria. 



\subsection{Computational Fluid Dynamics Model}

In previous modeling work, a three-dimensional CFD model was created and validated for flow simulations of the RPL effluent stack (Recknagle et al. 2008). The CFD model from this previous work included the ability to simulate the operation of both the scale-model and the full-scale final exhaust system with any number or combination of the four fans. For the present work, this model was exercised to simulate operation in each of the six permutations of two-fan operation, each fan operating at 52,000 $\mathrm{cfm}$ for a total of $104,000 \mathrm{cfm}$ air flow. Results of these simulations were then analyzed to determine the effectiveness of each fan combination to meet the ANSI/HPS N13.1-1999 criteria for well-mixed flow at the stack sampling location.

\subsection{Method}

The STAR-CD ${ }^{2}$ code was used to simulate the stack flow and analyze the simulation data to determine if the conditions at the sampling point would meet the criteria in ANSI/HPS N13.1-1999 for uniformity of air velocity, flow angularity, and uniformity of tracer gas and particles. In the calculations for the tests, STAR-CD solved the finite-volume Navier-Stokes (conservation of mass and momentum) and transport equations to obtain the steady-state flow field and species concentrations at each location within the system. For the sulfur hexafluoride tracer gas simulations, a Eulerian two-phase flow model was used in the calculations. A Lagrangian dispersed two-phase flow model was used for the aerosol (oil droplet) release simulations. The Lagrangian methodology includes models for droplet collision, breakup, drag, and turbulent dispersion of the dispersed phase (oil droplets). In all simulation cases, the generation and dissipation of turbulence was modeled using the $\kappa-\varepsilon$ turbulence model for large Reynolds number flow (as implemented in the STAR-CD code). The $\kappa-\varepsilon$ model is a widely tested and validated two-equation (partial differential equation) closure model for the turbulent transport terms in the time-averaged Navier-Stokes system of equations for fluid momentum transport. In the model equations, $\kappa$ is the turbulence kinetic energy, and $\varepsilon$ is the rate of dissipation of that energy. This turbulence model is considered suitable for the flow conditions present in such a duct. Additional details on the modeling approach can be obtained from Recknagle et al. (2008).

A three-dimensional model of the RPL final ventilation system was created to replicate the actual system geometry from the final exhaust fans to the stack exit (Figure 3.1). The view angle in the figure is slightly off vertical showing the horizontal orientation of the fan ducts and their perpendicular entry into the main duct (also horizontal), the $90^{\circ}$ horizontal turn of the main duct, and the turn from horizontal to the vertically oriented exhaust stack. Fan 1 is located furthest from the stack and fan number increases with proximity of the stack.

A simulation was run for each of the 2-fan configurations: fans $1 \& 2,1 \& 3,1 \& 4,2 \& 3,2 \& 4$, and $3 \& 4$. Data from each of simulations was extracted to obtain flow velocity, flow angle, concentration of tracer gas, and concentration of particles at each traverse point at the elevation of the sampling system. The data was entered into spreadsheets to determine whether the ANSI/HPS N13.1-1999 criteria would be met. Data sheets and plots are included in Appendix $\mathrm{H}$.

\footnotetext{
${ }^{2}$ The CFD program STAR-CD, Version 3.15, Methodology Volume, is copyrighted by the CD-adapco Group (CDadapco, Seattle Office, 3150 Richards, Suite 204, Bellevue, WA 98005).
} 


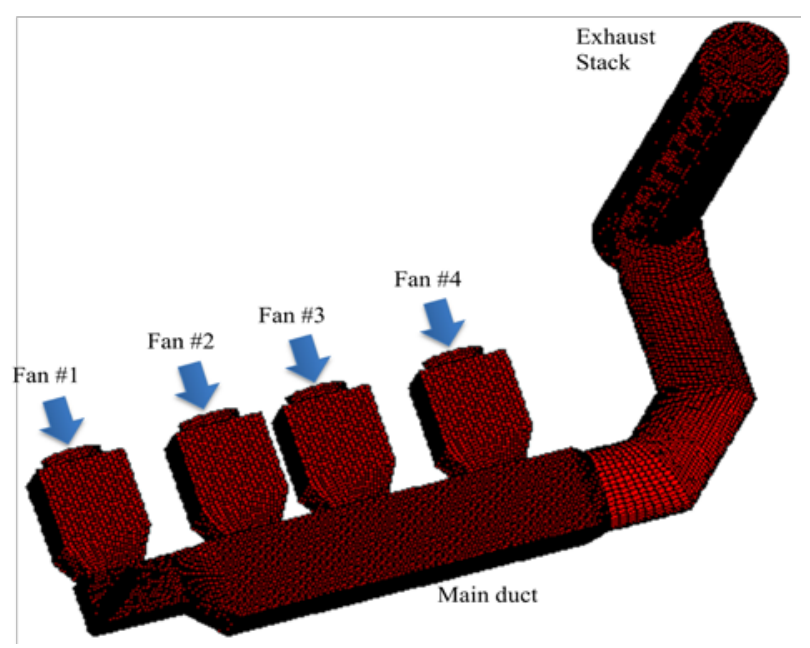

Figure 3.1. Three-Dimensional Model Geometry of the RPL Final Effluent Stack

\subsection{Results}

Detailed flow field results from the two-fan configuration case simulating operation with fans 1 and 2 are shown in Figure 3.2 and in Appendix H. The figure shows flow velocity vectors in which high velocity is red and low velocity is blue. Figure 3.2 shows the flow in a horizontal plane entering the duct through fans 1 and 2, passing the non-operating fans 3 and 4, turning the $90^{\circ}$ bend in the horizontal duct, and entering the vertical stack. Air from fan 1 was directed toward the main duct via turning vanes and joined by air from fan 2 in the main duct.

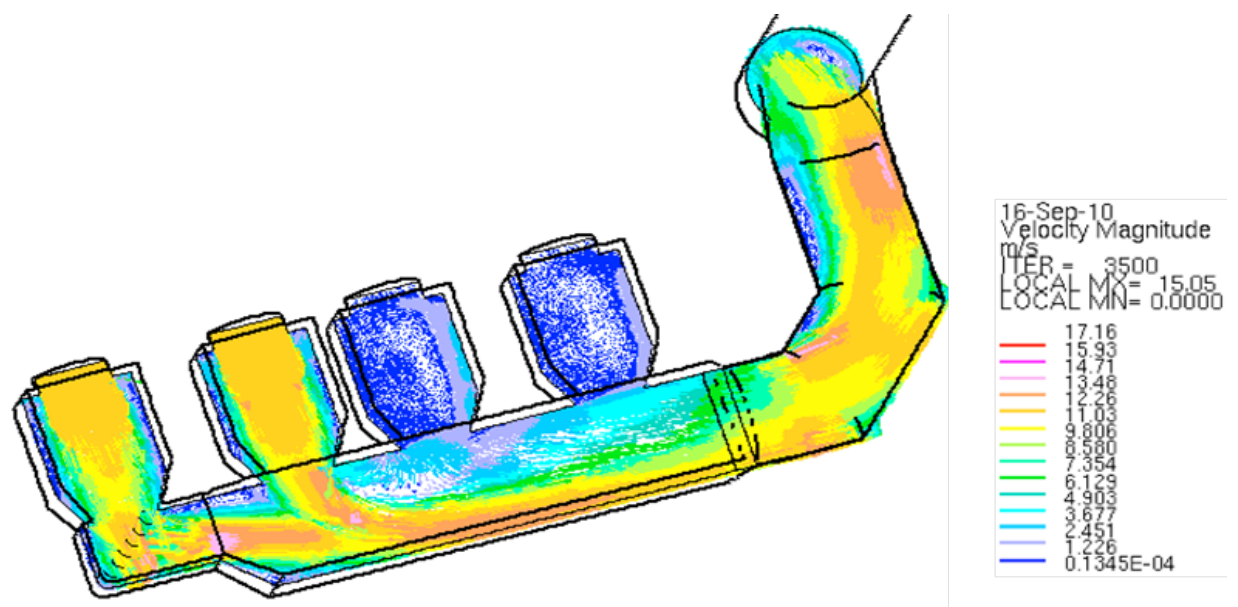

Figure 3.2. Flow Velocity Vectors in a Horizontal Plane (2-Fan Configuration, 104,000 cfm)

The transition from the horizontal duct to the vertical stack set up a swirl in the stack flow that can be seen in Figure 3.2 by the low velocity (blue) zone within the stack. A closer view of the horizontal section through the stack in Figure 3.3 shows the circulation zone at upper right with relatively higher speed flow surrounding the zone. This swirl was present in each flow case and contributed to the mixing within the stack. 


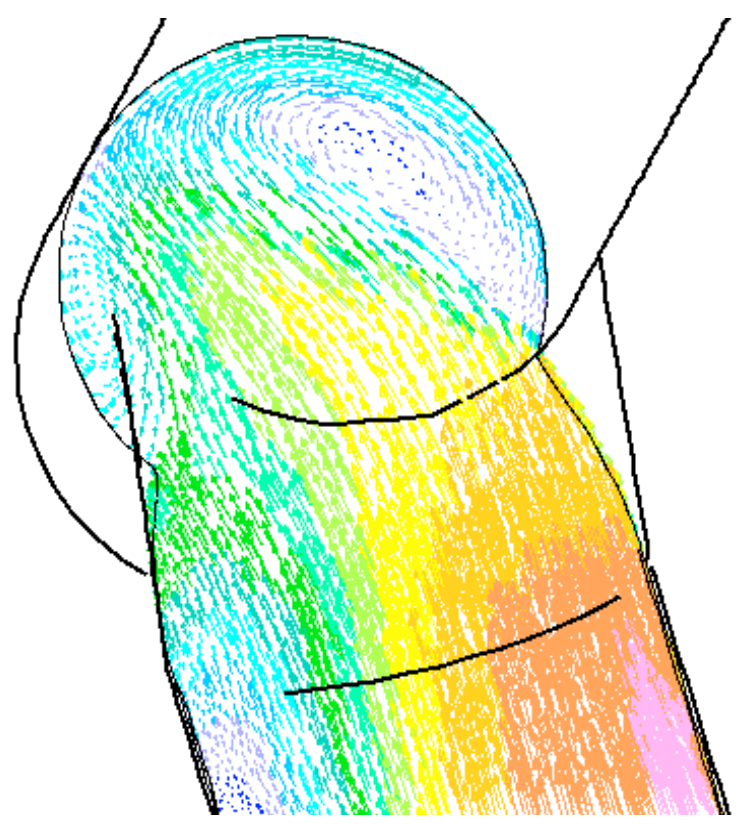

Figure 3.3. Detail of Flow Vectors in a Horizontal Plane

Results of each of the two-fan configuration simulations are shown in Table 3.1, along with criteria from ANSI/HPS N13.1-1999. As shown in the table, all criteria are met in each of the simulations.

Table 3.1. Results from CFD Modeling of RPL Final Exhaust System

\begin{tabular}{lccccc}
\hline & $\begin{array}{c}\text { Velocity } \\
\text { Uniformity } \\
(\text { COV \%) }\end{array}$ & $\begin{array}{c}\text { Cyclonic } \\
\text { Flow } \\
(\text { Angle }\end{array}$ & $\begin{array}{c}\text { Gas Tracer } \\
\text { Uniformity } \\
(\text { COV \%) }\end{array}$ & $\begin{array}{c}\text { Gas Tracer } \\
\text { Uniformity } \\
\left(\begin{array}{c}\text { max deviation from } \\
\text { and \% of mean) }\end{array}\right.\end{array}$ & $\begin{array}{c}\text { Aerosol } \\
\text { Uniformity } \\
\text { (COV \% } \\
\text { normalized })\end{array}$ \\
\hline 1,2 & 5.75 & 11.4 & 6.83 & 13.8 & 6.72 \\
1,3 & 5.83 & 11.2 & 6.18 & 13.3 & 7.60 \\
1,4 & 5.84 & 12.4 & 9.29 & 23.2 & 10.4 \\
2,3 & 5.61 & 10.8 & 6.22 & 14.3 & 7.12 \\
2,4 & 6.00 & 11.5 & 8.80 & 20.3 & 10.8 \\
3,4 & 6.02 & 11.4 & 8.74 & 20.4 & 10.9 \\
ANSI/HPS N13.1-1999 criteria & $\leq 20$ & $\leq 20$ & $\leq 20$ & $\leq 30$ & $\leq 20$ \\
\hline
\end{tabular}





\subsection{Conclusions}

A scale model was designed following criteria in ANSI/HPS N13.1-1999 of the 325 Building RPL final exhaust system. The scale model was used to determine whether the stack sampling system would meet criteria in ANSI/HPS 13.1-1999 for sampling system location under reduced exhaust flow conditions. A summary of the tests, measurement results, and criteria are provided in Table 4.1 and demonstrate that the sampling location on the 325 Building main stack meets the criteria in ANSI/HPS N13.1-1999 for a well mixed location during the set-back flow condition when only one fan is in operation.

Table 4.1. Summary of Test Results for Low Flow RPL Exhaust

\begin{tabular}{lcccc}
\hline \multicolumn{1}{c}{ Test } & $\begin{array}{c}\text { Scale-Model } \\
\text { Measurement } \\
(1-f a n)\end{array}$ & $\begin{array}{c}\text { Full-Scale } \\
\text { Measurement } \\
(1-f a n)\end{array}$ & $\begin{array}{c}\text { CFD } \\
\text { Simulation } \\
\text { Results } \\
(2-f a n)\end{array}$ & $\begin{array}{c}\text { ANSI/HPS } \\
\text { N13.1-1999 } \\
\text { Criteria }\end{array}$ \\
\hline Velocity uniformity (COV \%) $^{\text {Cyclonic flow (angle }}{ }^{\circ}$ ) & $1.6-9.4$ & $3.9-10.7$ & $5.6-6.0$ & $\leq 20$ \\
Gas tracer uniformity (COV \%) $_{\text {Gas tracer uniformity (maximum deviation }}^{8.0-14.3}$ & & $10.8-12.4$ & $\leq 20$ \\
from and \% of the mean) & $1.6-5.5$ & & $6.2-9.3$ & $\leq 20$ \\
Aerosol uniformity (COV \%, normalized) & $2.6-12.6$ & & $13.3-23.2$ & $\leq 30$ \\
\hline
\end{tabular}

With regard to acceptance of the scale model as a substitute for the actual stack, the model used was designed to be geometrically similar to the actual stack. Components influencing contaminant mixing and velocity profile in the scale model were proportional to those in the actual stack, and the sampling location in the scale model placed in a geometrically similar location as in the actual stack. The velocity profile in the actual stack meets the uniformity criteria, and the difference between velocity COVs of the two systems is not more than 5\% COV units (Table 4.1). The scale model stack diameter of 18 inches is greater than the minimum of $250 \mathrm{~mm}$ (10 in).

The lowest flow used in the scale model testing was 1,100 cfm (Table 1.1) resulting in a Reynolds number of 91,000 which is well above the required minimum of 10,000. Reynolds number calculations are shown in Appendix $\mathrm{G}$ for the ranges of flows in the actual and scale model stacks under normal and reduced flow conditions. All Reynolds numbers are substantially above 10,000.

Finally, the scale model's mean velocity times hydraulic diameter must be within a factor of six of the actual stack. For the one fan configuration, the scale model's mean velocity times hydraulic diameter at the $37.1 \mathrm{~Hz}$ setting ranged from $930-1200 \mathrm{ft}^{2} / \mathrm{min}$. Using a factor of six, this corresponds to stack flows of 980-45,400 cfm (calculations in Appendix G).

All combinations of two-fan configurations were evaluated using a three-dimensional CFD model. The CFD simulation results are shown in Table 4.1 and indicate that the stack sampling system also meets ANSI/HPS N13.1-1999 criteria for a well mixed location with any two fans operating.

The scale-model measurement tests cover the reduced operating range of flows less than 45,400 cfm using a one fan configuration. Earlier tests were conducted on normal flow conditions covering three fan configurations ranging from 129,000 to 186,300 cfm (Smith et al. 2010). The three-dimensional CFD simulations were performed to evaluate all two-fan configurations. All measurements and simulations results showed that the sampling system location in the RPL stack meets the ANSI/HPS N13.1-1999 criteria for flow angularity and uniformity of flow, tracer gas, and aerosol under one-, two-, or three-fan configurations and flow conditions. 



\subsection{References}

ANSI-American National Standards Institute. 1969. Guide to Sampling Airborne Radioactive Materials in Nuclear Facilities. ANSI N13.1-1969, American National Standards Institute, NY. February 19.

ANSI/HPS-American National Standards Institute/Health Physics Society. 1999. Sampling and Monitoring Releases of Airborne Radioactive Substances From the Stacks and Ducts of Nuclear Facilities. ANSI/HPS N13.1-1999, American National Standards Institute, NY.

Ballinger MY, JM Barnett, JA Glissmeyer, and DL Edwards. 2004. "Evaluation of Sampling Locations for Two Radionuclide Air-Sampling Systems Based on the Requirements of ANSI/HPS N13.1-1999." Health Physics 86(4):406-415.

EPA-U.S. Environmental Protection Agency. 40 CFR 60, Appendix A, Method 1, as amended. "Method 1 - Sample and Velocity Traverses for Stationary Sources.” Code of Federal Regulations.

Recknagle KP, ST Yokuda, MY Ballinger, and JM Barnett. 2008. "Scaled Tests and Modeling of Effluent Stack Sampling Location Mixing.” Health Physics 96(2):164-174.

Smith BM, MY Ballinger, JA Glissmeyer, and JM Barnett. 2010. Sampling Point Compliance Tests for the 325 Building Stack at Full Flow Condition. PNNL-19998, Pacific Northwest National Laboratory, Richland, WA. 

Appendix A

\section{Test Plan for Qualifying the EP-325-01-S Stack Air Sampling Position for Set-Back Flow Condition}





\section{Test Plan for Qualifying the EP-325-01-S Stack Air Sampling Position for Set-Back Flow Condition August 10, 2004}

This series of tests will demonstrate whether the EP-325-01-S exhaust stack meets the applicable regulatory criteria regarding the placement of the air-sampling probe. This has already been demonstrated for the normal flowrate with three operating fans. This re-test will demonstrate whether the criteria are still met if the system flowrate is reduced from the normal value by a factor of three when only one fan is used. This stack exhausts the filtered ventilation air from all areas of the 325 Building Radiochemical Processing Laboratory where radionuclides are handled, including hot cell, waste treatment and radiochemistry. The tests will be conducted by Pacific Northwest National Laboratory staff. The standard governing the performance of the tests, test methods, and acceptance criteria is ANSI/HPS N13.1-1999, Sampling and Monitoring Releases of Airborne Radioactive Substances From the Stack and Ducts of Nuclear Facilities.

\section{Performance Criteria}

The qualification criteria for the location of the air sampling probe are as follows (Table 4, ANSI/HPS N13.1-1999):

1. Flow Angle - Sampling nozzles are usually aligned with the axis of the stack. If the air travels up the stack in cyclonic fashion, the air velocity vector approaching the nozzle could be misaligned with the sampling nozzles enough to impair the extraction of particles. Consequently, the flow angle is measured in the stack at the elevation of the sampling nozzle. The average air-velocity angle must not deviate from the axis of the sampling nozzle by more than $20^{\circ}$.

2. Air Velocity Uniformity - It is important that the gas momentum across the stack cross section where the sample is extracted be well mixed or uniform. Consequently, the velocity is measured at several points in the stack at the elevation of the sampling nozzle. The uniformity is expressed as the variability of the measurements about the mean. This is expressed using the relative coefficient of variance (COV), which is the standard deviation divided by the mean and expressed as a percentage. The lower the COV value, the more uniform the velocity. The acceptance criteria is that the COV of the air velocity must be $\leq 20 \%$ across the center two-thirds of the area of the stack.

3. Gas Tracer Uniformity - A uniform contaminant concentration in the sampling plane enables the extraction of samples that represent the true concentration. This is first tested using a tracer gas to represent gaseous effluents. The fan is a good mixer, so injecting the tracer downstream of the fan provides worst-case results. The acceptance criteria are that 1 ) the COV of the measured tracer gas concentration is $\leq 20 \%$ across the center two-thirds of the sampling plane and 2 ) at no point in the sampling plane does the concentration vary from the mean by $>30 \%$.

4. Particle Tracer Uniformity - Uniformity in contaminant concentration at the sampling elevation is further demonstrated using tracer particles large enough to exhibit inertial effects. Particles of $10-\mu \mathrm{m}$ aerodynamic diameter $(\mathrm{AD})$ are used by default unless it is known that larger particles are present in the airstream. The acceptance criteria is that the COV of particle concentration is $\leq 20 \%$ across the center two-thirds of the sampling plane. 


\section{Scale Model Testing Criteria}

Testing to satisfy Criteria $1-4$ will be conducted on a scale model of the exhaust ductwork and stack, from the heat recovery coils to the elevation of the sampling probe. The ANSI/HPS N13.1-1999 standard sets acceptance criteria for the use of a scale model as a substitute for the actual stack.

- The scale model and its sampling location must be geometrically similar to the actual stack.

- The model's mean velocity $x$ hydraulic diameter product will be within a factor of six of the actual stack.

- $\quad$ The Reynold's number for the prototype and model stacks must $>10,000$.

The scale model results are considered valid if:

- The velocity profile in the actual stack meets the uniformity criteria, and

- The uniformity COV for the actual and model stacks agree within 5\% COV.

\section{Conduct of Tests}

Compliance with each performance criteria is demonstrated through performing a specific test procedure. The four procedures to be used are briefly described below and in the order in which they are usually conducted. Specific Test Instructions will be issued prior to the conduct of a test.

The tracer tests result in the emission of tracer gas (sulfur hexafluoride) and tracer particles (vacuum pump oil mist) from the scale model. The estimate of emissions from these tests is given below.

The Job Hazard Analysis (if any) and MSDS's for the tracer compounds are to be reviewed by testing staff prior to the conduct of any test. These documents are also to be kept available at the test site.

\subsection{Flow Angle Test}

The air-velocity vector approaching the sample nozzle should be aligned with the axis of the nozzle, within an acceptable angle, so sample extraction performance is not degraded. The test method is based on 40 CFR 60, Appendix A, Method 1, Section 2.4, "Verification of the Absence of Cyclonic Flow."

This test is conducted on the scale model stack. The flow angle is measured at a grid of points in a cross section of the stack at the scaled elevation of the actual sampling probe. The grid is an array of points in an x-pattern. One line of points is aligned in the same direction as the existing sampling probe on the actual stack. The other line will be perpendicular to that. The number and distance between measurement points is based on the EPA procedure 40 CFR 60, Appendix A, Method 1.

Measurements are made using a type-S pitot tube, a slant tube or electronic manometer, and a protractor level or angle indicating device attached to the pitot tube. The procedure EMS-JAG-05 Test to Determine Flow Angle at the Elevation of a Sampler Probe provides the general procedure for the determination of mean flow angle. Instructions specific for this stack will be given in a test instruction. 


\subsection{Air Velocity Uniformity Test}

The uniformity of air velocity where the air sample is being extracted ensures that the air momentum in the stack is well mixed. To determine uniformity, air velocity is measured at the same grid of points used for the angular flow test. The method used is based on 40 CFR 60, Appendix A, Method 1. The equipment includes a standard Prandtl-type or S-type pitot tube and a calibrated electronic manometer. Procedure EMS-JAG-04, Test to Determine Uniformity of Gas Velocity at the Elevation of a Sampler Probe, is used for this test. This test takes about 90 minutes. A test instruction will be issued specifically for this test.

\subsection{Gas Tracer Uniformity Test}

A uniform contaminant concentration in the sampling plane enables the extraction of samples that represent the true concentration. Procedure EMS-JAG-01, Test to Determine Uniformity of a Tracer Gas at a Sampler Probe, is used for this test. The gaseous contaminant concentration uniformity is demonstrated using a tracer gas (sulfur hexafluoride) injected into the ductwork at various positions. For each injection position, the tracer concentration is measured at the sampling plane at the same grid of measurement points as used for the above tests. The uniformity is expressed as the COV of the measured tracer concentrations.

Tracer gas concentration is measured with a Bruel and Kjaer (Naerum, Denmark) Model 1302 gas analyzer calibrated for the tracer gas. The absolute calibration of the analyzer is unimportant to the test results; however, the analyzer response is checked using calibration standards prior to the conduct of the test. If the indicated concentration is within $20 \%$ of the standard, the response is acceptable.

For the proposed reduced flow condition, any one of the available four fans would be used to maintain the reduced airflow. There is a heat recovery box between each fan and the final exhaust duct. The planned tracer gas injection locations are just downstream of the heat recovery boxes nearest and farthest from the stack. These should represent the best and worst case conditions. An optional location would be in the duct just upstream of the breach to the stack. The tracer is injected along the centerline of the duct and at four locations near the corners of the duct.

For the single operating fan configuration, the five tracer injection points will be used for each injection location. The test will be repeated for the injection point found to have the least favorable result. Other repeat runs may be made at the discretion of the test director after a review of the preliminary results. Therefore, there will be a minimum of 11 test runs. Each run takes about 90 minutes. A test instruction will be issued specifically for this test.

The usage and emission of the tracer gas, sulfur hexafluoride, is based on a stack flow of $1442 \mathrm{cfm}$, a desired concentration of $1 \mathrm{ppm}$, and 13 tests. The total emission would be about $0.30 \mathrm{~kg}$ for 13 tests, or about $0.022 \mathrm{~kg} / \mathrm{test}$.

\subsection{Particle Tracer Uniformity Test}

The test for uniformity of tracer particles is similar to the test for uniformity of tracer gases. The general approach is to inject particles of a range of sizes, including the size of interest, into the center of the airstream. The concentration of the particles of the size range of interest is then measured at several points in the cross section of the sampling plane using an optical particle counter (OPC, Met-One Model A2408, Grants Pass, Oregon). A simple probe is used to extract the sample and deliver it to the OPC. The measurement points are the same as used for the above tests. The OPC should be within calibration. 
The particles are made by spraying vacuum-pump oil through a nozzle housed in a chamber. The chamber provides a controlled means for injecting the particles into the airflow through a probe. Compressed air and an injection probe are required for the operation of the chamber.

The tracer injection port is the same as for the gaseous tracer; however, only the centerline injection point is used. The layout of measurement points is the same as for all of the other tests, except where the size of the probe does not permit sampling as close to the inside of the stack wall.

The OPC's sort the number of particles into six size channels. Each concentration reading is the count of particles collected in the 9- to $11-\mu \mathrm{m}$ channel. The readings are recorded on a data sheet. Three readings are taken at each point and averaged. The coefficient of variance of the average concentration readings at all points is calculated and the result compared to the acceptance criteria for uniformity. The particle mixing is acceptable if the COV of the tracer particles of $10-\mu \mathrm{m}$ aerodynamic diameter (AD) is less than or equal to $20 \%$ across the center two-thirds of the sampling plane.

There will be a minimum of 5 test runs, one for each injection position (used for the gas tracer uniformity tests) and flowrate, and one repeat test. Each test run can require up to four hours. Procedure EMS-JAG02, Test to Determine Uniformity of a Tracer Aerosol at a Sampler Probe, is used for this test. A test instruction will be issued specifically for this test.

The usage and emission of the tracer aerosol oil (Fisherbrand 19 vacuum pump oil) may be as about high as $50 \mathrm{~g}$ per test run. (The use rate has never actually been measured. In any demonstrations that we have done, it has always been less than $0.5 \mathrm{~L}$ total.) Three tests can be completed in an 8-hour period.

\section{Fan Configuration}

In the actual fan house, there are four electric fans, three of which are normally used at a time. The capacity of the electric fans are about $46,000 \mathrm{cfm}$ each. With three fans operating, the exhaust airflow is about $138,000 \mathrm{cfm}$. With one fan running in the reduced flow condition, the exhaust airflow would be about 46,000 cfm. The fans are housed in a small building. Each fan discharge's upward and into a heat recovery box, where coils and heat transfer fluid recover heat from the exhaust airflow. Each heat recovery box discharges into the main plenum which connects to the stack. Figure 1 is a diagram of the scale model for this part of the ventilation system. The parameters for the scale model are given in Table 1. The 5.33:1 scale results in a convenient stack and duct size of 18 inches to represent the actual 8 feet. It also results in a velocity close to that of the actual system. (With three fans operating, the actual and model velocities would be 2755 and 2449 fpm respectively.) To remain within the scaling parameters (velocity times hydraulic diameter within a factor of six) the model flowrate of $1442 \mathrm{cfm}$ corresponds to $46,157 \mathrm{cfm}$, or one-third the stack flowrate of 138,473 (the average of eleven separate stack flow measurements).

There are plans to upgrade the fan capacities to about 50,000 or $60,000 \mathrm{cfm}$ each. That would be a flowrate around 50,000 - 240,000 cfm depending on the fan usage. The overall flowrate range is then 45,666 (based on the lowest 3-fan stack flowrate measured) - 240,000 cfm. The corresponding minimum scale model flowrates would then be $1427-7500 \mathrm{cfm}$. 
Table 1. Possible Scale Model Parameters

Flow parameters in scale models

(colored values are inputs)

John Glissmeyer 4/13/01

Fluid characteristics:

Mol. Wt.

Temperature

Pressure

Gas Density

Viscosity

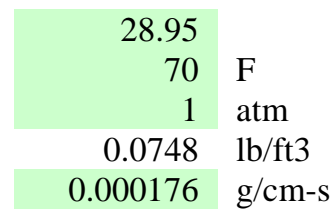

Range of Reynolds number for scale model:

$\begin{array}{lll}\text { Min } & 3.88 \mathrm{E}+05 & 1 / 6 \text { prototype } \\ \text { Max } & 1.40 \mathrm{E}+07 & 6 \mathrm{X} \text { prototype }\end{array}$

Range of DV for scale model:

$\begin{array}{rrr}\text { Min } & 3.67 \mathrm{E}+03 & 1 / 6 \text { prototype } \\ \text { Max } & 1.32 \mathrm{E}+05 & 6 \text { X prototype } \\ \text { Prototype } & 2.20 \mathrm{E}+04 & \end{array}$

Sheet protected for data entry, no password

Want to keep velocity < Mach 0.3, or $<19700 \mathrm{fpm}$

Prototype Stack characteristics:

$\begin{array}{lrll}\text { Diam } & 8.00 & \mathrm{ft} \\ \text { Flow } & 138473 & \mathrm{cfm} \\ \text { X-area } & 50.265 & \mathrm{sq} . \mathrm{ft} \\ \text { Mean U } & \mathbf{2 7 5 5} & \mathrm{ft} / \mathrm{min} \\ \text { Reynolds } & \mathbf{2 . 3 3 E + 0 6} & \end{array}$

Range of $\mathbf{Q} / \mathbf{D}$ for scale model:

Min $\quad 2.88 \mathrm{E}+03 \quad 1 / 6$ prototype

Max $1.04 \mathrm{E}+056 \mathrm{X}$ prototype

Prototype $1.73 \mathrm{E}+04$

\begin{tabular}{|c|c|c|c|c|c|c|c|}
\hline \multirow[b]{2}{*}{ Section } & \multirow[b]{2}{*}{$\begin{array}{l}\text { Prototype } \\
\text { ft }\end{array}$} & \multicolumn{6}{|c|}{ Scaled ft at $1: X$} \\
\hline & & 3.20 & 4.00 & 5.33 & 7.00 & 1.00 & 1.00 \\
\hline Round Duct Section & Stack & & & & & & \\
\hline Diam, ft & 8.00 & 2.500 & 2.000 & 1.500 & 1.143 & 8.000 & 8.000 \\
\hline Area, ft2 & 50.3 & 4.91 & 3.1 & 1.8 & 1.0 & 50.3 & 50.3 \\
\hline Flow at $\min Q / D, c f m$ & 138473 & 7212 & 5770 & 4327 & 3297 & 23079 & 23079 \\
\hline Vel fpm & 2755 & 1469 & 1837 & 2449 & 3214 & 459 & 459 \\
\hline $\mathrm{Re}$ & $2.3 \mathrm{E}+06$ & $3.9 \mathrm{E}+05$ & $3.9 E+05$ & $3.9 \mathrm{E}+05$ & $3.9 \mathrm{E}+05$ & $3.9 \mathrm{E}+05$ & $3.9 \mathrm{E}+05$ \\
\hline Ratio Re & & 6.00 & 6.00 & 6.00 & 6.00 & 6.00 & 6.00 \\
\hline Round Duct Section & Stack & & & & & & \\
\hline Diam, ft & 8.00 & 2.50 & 2.00 & 1.50 & 1.14 & 8.00 & 8.00 \\
\hline Area, ft2 & 50.3 & 4.91 & 3.1 & 1.8 & 1.0 & 50.3 & 50.3 \\
\hline Flow at $\min Q / D, c f m$ & 45666 & 2378 & 1903 & 1427 & 1087 & 7611 & 7611 \\
\hline Vel fpm & 908 & 485 & 606 & 808 & 1060 & 151 & 151 \\
\hline $\mathrm{Re}$ & $2.3 \mathrm{E}+06$ & $1.3 \mathrm{E}+05$ & $1.3 \mathrm{E}+05$ & $1.3 \mathrm{E}+05$ & $1.3 \mathrm{E}+05$ & $1.3 \mathrm{E}+05$ & $1.3 \mathrm{E}+05$ \\
\hline Ratio Re & & 18.19 & 18.19 & 18.19 & 18.19 & 18.19 & 18.19 \\
\hline Round Duct Section & Stack & & & & & & \\
\hline Diam, ft & 8.00 & 2.500 & 2.000 & 1.500 & 1.143 & 8.000 & 8.000 \\
\hline Area, ft2 & 50.3 & 4.91 & 3.1 & 1.8 & 1.0 & 50.3 & 50.3 \\
\hline Flow at $\min \mathrm{Q} / \mathrm{D}, \mathrm{cfm}$ & 240000 & 12500 & 10000 & 7500 & 5714 & 40000 & 40000 \\
\hline Vel fpm & 4775 & 2546 & 3183 & 4244 & 5570 & 796 & 796 \\
\hline $\operatorname{Re}$ & $2.3 E+06$ & $6.7 \mathrm{E}+05$ & $6.7 \mathrm{E}+05$ & $6.7 \mathrm{E}+05$ & $6.7 \mathrm{E}+05$ & $6.7 \mathrm{E}+05$ & $6.7 \mathrm{E}+05$ \\
\hline Ratio Re & & 3.46 & 3.46 & 3.46 & 3.46 & 3.46 & 3.46 \\
\hline Round Duct Section & Stack & & & & & & \\
\hline Diam, ft & 8.00 & 2.500 & 2.000 & 1.500 & 1.143 & 8.000 & 8.000 \\
\hline Area, ft2 & 50.3 & 4.91 & 3.1 & 1.8 & 1.0 & 50.3 & 50.3 \\
\hline Flow at $\min \mathrm{Q} / \mathrm{D}, \mathrm{cfm}$ & 60000 & 3125 & 2500 & 1875 & 1429 & 10000 & 10000 \\
\hline Vel fpm & 1194 & 637 & 796 & 1061 & 1393 & 199 & 199 \\
\hline $\operatorname{Re}$ & $2.3 \mathrm{E}+06$ & $1.7 \mathrm{E}+05$ & $1.7 \mathrm{E}+05$ & $1.7 \mathrm{E}+05$ & $1.7 \mathrm{E}+05$ & $1.7 \mathrm{E}+05$ & $1.7 \mathrm{E}+05$ \\
\hline Ratio Re & & 13.85 & 13.85 & 13.85 & 13.85 & 13.85 & 13.85 \\
\hline
\end{tabular}




\section{Test Runs}

Table 2 lists the estimated number of runs of the individual tests that will be performed for the reduced flow configuration.

Table 2. Minimum Test Runs to be Performed for the Reduced Flow Configuration

\begin{tabular}{|l|l|l|l|l|l|}
\hline \multicolumn{2}{|c|}{} & \multicolumn{4}{|c|}{ Estimated number of test runs } \\
\hline Configuration & $\begin{array}{l}\text { Scaled Stack } \\
\text { Flowrate }\end{array}$ & Flow Angle & Air Velocity & Gas Tracer & $\begin{array}{l}\text { Particle } \\
\text { Tracer }\end{array}$ \\
\hline Current Fans & 1427 & 1 & 2 & 11 & 3 \\
\hline
\end{tabular}

All measurements are planned to be conducted at the simulated sampling probe elevation of the stack. The probe centerline is about 55.94- $\mathrm{ft}$ (?) above the top stack breach. That should be $10.5 \mathrm{ft}$ on the scale model.

The test strategy underlying Table 2 is outlined in Table 3.

Table 3. Proposed Test Sequence

\begin{tabular}{|c|c|c|c|}
\hline Test & Run & Injection Points & Comments \\
\hline \multicolumn{4}{|c|}{ One Fan Configuration } \\
\hline Flow Control Cal. & VT-1, VF-1 & N.A. & $\begin{array}{l}\text { VT-1 at } 30 \mathrm{~Hz} \text { on } \\
\text { controller, VF-1 at 5- } \\
\text { Hz increments }\end{array}$ \\
\hline Velocity Uniformity & $\begin{array}{l}\text { VT-2, VT-3, VT-4 } \\
\text { and VT-5 }\end{array}$ & N.A. & $\begin{array}{l}\text { First two where fan } \\
\text { closest to the stack is } \\
\text { used. Second two } \\
\text { where the farthest fan } \\
\text { is used }\end{array}$ \\
\hline Flow Angle & FA-1, FA-2, FA-3 & N.A. & $\begin{array}{l}\text { One run per condition } \\
\text { plus one repeat }\end{array}$ \\
\hline \multirow[t]{3}{*}{ Gas Tracer } & GT-1 to GT-5 & $\begin{array}{l}\text { With injection } \\
\text { downstream of Fan } 4\end{array}$ & $1427 \mathrm{cfm}$ \\
\hline & GT-6 to GT-10 & $\begin{array}{l}\text { With injection } \\
\text { downstream of Fan } 1\end{array}$ & $1427 \mathrm{cfm}$ \\
\hline & GT-11 & $\begin{array}{l}\text { Repeat of worst case } \\
\text { from above }\end{array}$ & $1427 \mathrm{cfm}$ \\
\hline Particle Tracer & PT-1 to PT-3 & $\begin{array}{l}\text { Centerline after Fan } 1 \\
\text { and } 4 \text { and repeat of } \\
\text { worst case }\end{array}$ & $1427 \mathrm{cfm}$ \\
\hline
\end{tabular}


Figure 1. Scale Model Drawing for 325 Stack
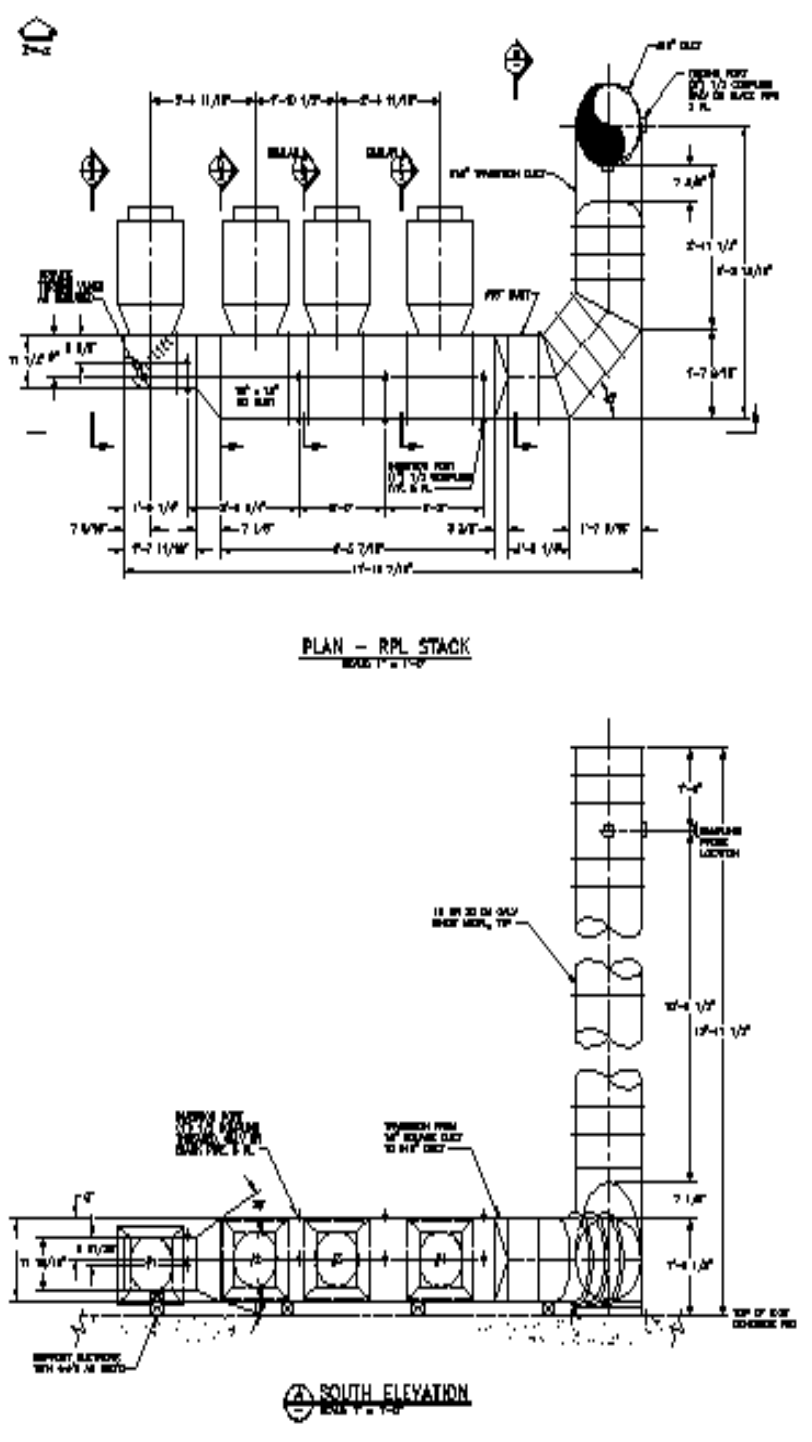

Appendix B

Flow Calibration Procedure and Data 



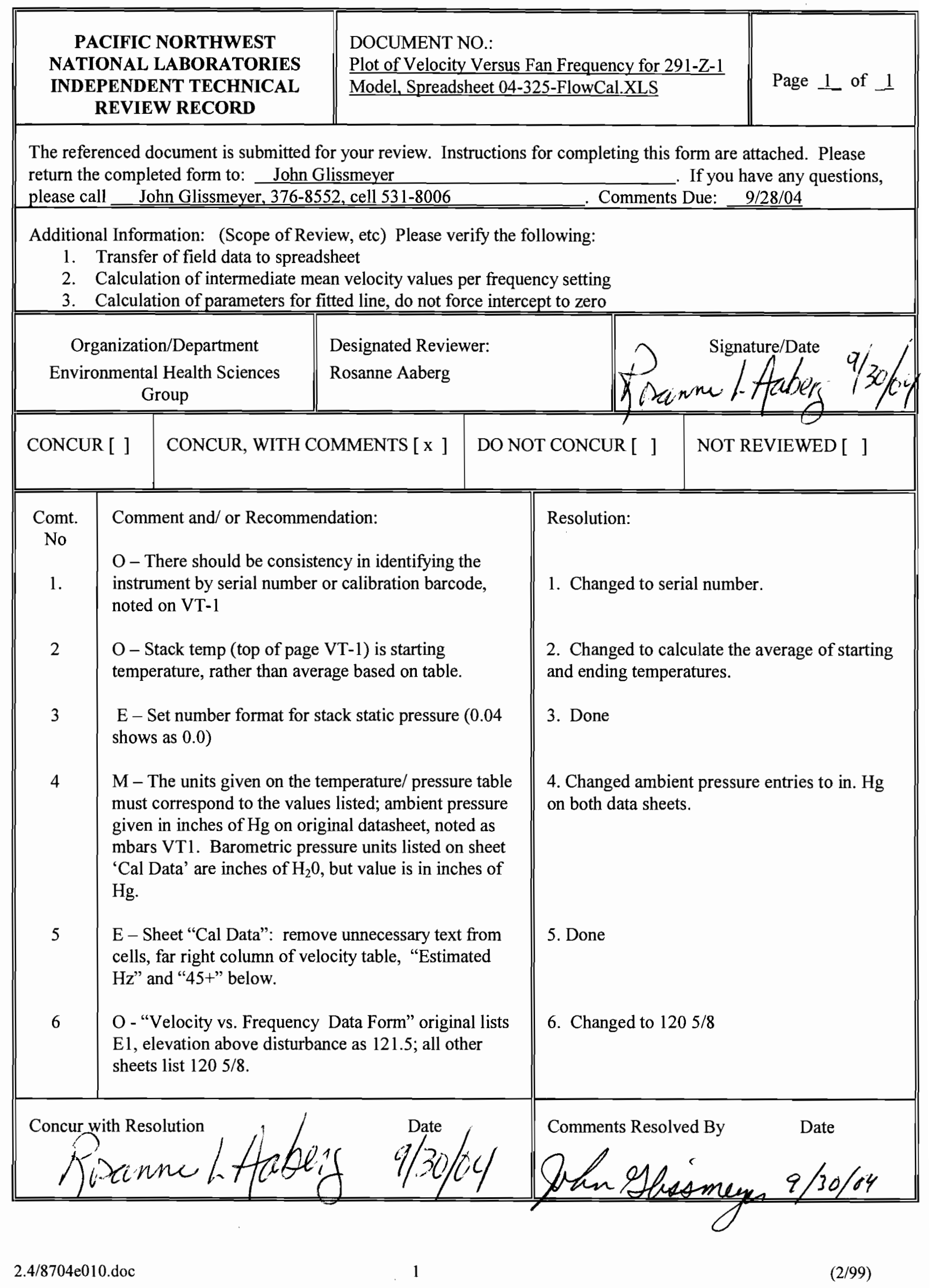


VELOCITY TRAVERSE DATA FORM

Site 325 Model

Date 8/19/2004

Testers JMB/GSH/MYB/DDD

Stack Dia.

Stack X-Area

Elevation

$\frac{18 \text { in. }}{-254.5 \text { in. } 2}$

Jistance to disturbance $1205 / 8$ inches

Velocity units fumin
Run No. VT-LOW1

Fan Configuration FAN 4 (near stack)

Fan Setting $30 \mathrm{HZ}$

Stack Temp

$95.6 \operatorname{deg} \mathrm{F}$

Start/End Time 12:19PM - 2:20PM

Center $2 / 3$ from 1.65 to: 16.35

Points in Center $2 / 3$

Data Files: NA

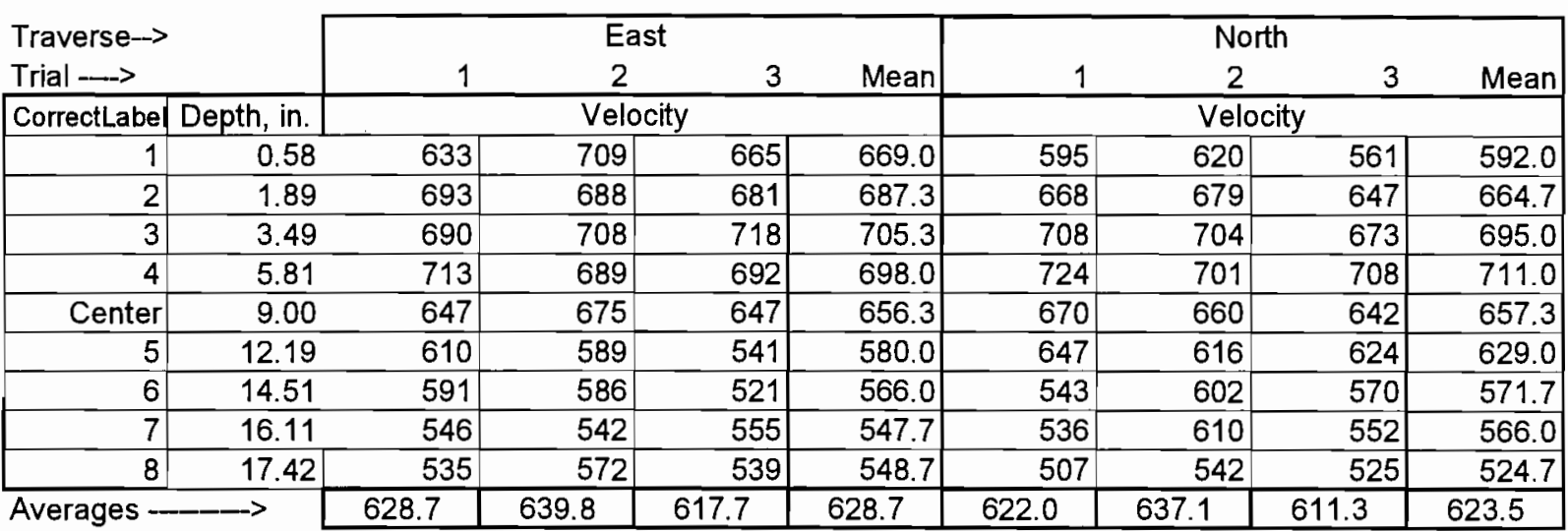

\begin{tabular}{|c|c|c|c|c|c|c|}
\hline$A \mid I$ & $\mathrm{ft} / \mathrm{min}$ & Dev. from mean & Center 2/3 & West & North & All \\
\hline Mean & $\overline{626.1}$ & & Mean & 634.4 & 642.1 & 638.2 \\
\hline Min Point & 524.7 & $-16.2 \%$ & Std. Dev. & 67.7 & 56.6 & 60.1 \\
\hline Max Point & 711.0 & $13.6 \%$ & COV as $\%$ & 10.7 & 8.8 & 9.4 \\
\hline
\end{tabular}

Flow w/o C-Pt 1100 acfm

Vel Avg w/o C-Pt $622 \mathrm{fpm}$

Stack temp

Equipment temp

Ambient temp

Stack static

Ambient pressure

Total Stack pressure

Ambient humidity

\begin{tabular}{|c|c|c|}
\hline Start & Finish & \\
\hline 98 & 93.2 & $F$ \\
\hline 87 & 88.7 & $F$ \\
\hline 87 & 92 & $F$ \\
\hline 0.04 & 0.03 & mbars \\
\hline 29.4 & 29.4 & in $\mathrm{Hg}$ \\
\hline 1013.1 & 1012.2 & mbars \\
\hline $37 \%$ & $31 \%$ & $\mathrm{RH}$ \\
\hline
\end{tabular}

Instuments Used:

Solomat Zephyr SN 1295-1472

Cal $8 / 12 / 02$

Notes: $\quad$ Wind ENE $6 \mathrm{mph}$
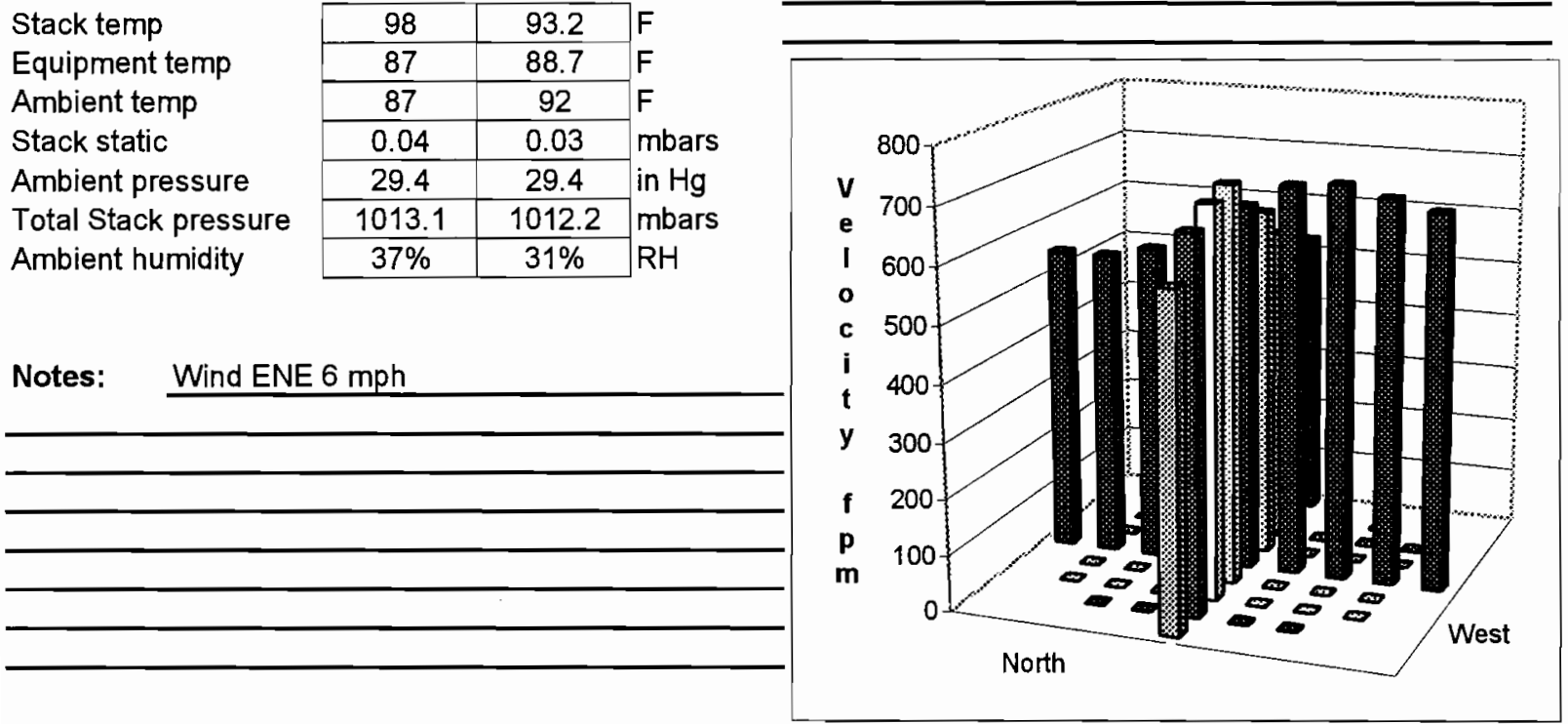

Signature signifies compliance with Signature verifying data and calgulations: Procedure EMS Signature/date

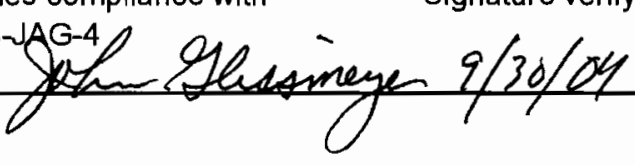

\section{III .}




\section{VELOCITY vS. FREQUENCY DATA FORM}

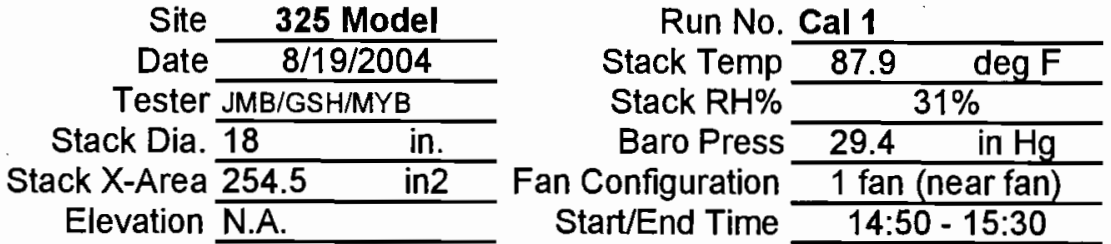

El. above disturbance $\overline{1205 / 8 \text { inches }}$ Reference point from velocity test VT North 5 Velocity Readings, units $=$ fpm

\begin{tabular}{|c|c|c|c|c|c|c|c|}
\hline & \multirow{4}{*}{$\begin{array}{r}\begin{array}{r}\text { Target } \\
\text { cfm }\end{array} \\
1350 \\
1450\end{array}$} & \multirow{4}{*}{\begin{tabular}{|r|}
$\begin{array}{r}\text { Target } \\
\text { fpm }\end{array}$ \\
764 \\
821 \\
\end{tabular}} & \\
\hline & & & & & & & \\
\hline & & & & & & & \\
\hline & \multicolumn{4}{|c|}{ fpm } & & & \\
\hline $\mathrm{Hz}$ & 1 & 2 & 3 & Mean & StDev & 2 StDev & $\mathrm{cfm}$ \\
\hline 5 & 32 & 0 & 22 & 18.00 & 16.37 & \begin{tabular}{|l|}
32.74 \\
\end{tabular} & 31.81 \\
\hline 10 & 150 & 138 & 177 & 155.00 & 19.97 & 39.95 & 273.91 \\
\hline 15 & 320 & 266 & 272 & 286.00 & 29.60 & 59.19 & 505.40 \\
\hline 20 & 420 & 406 & 400 & 408.67 & 10.26 & 20.53 & 722.17 \\
\hline 25 & 536 & 490 & 513 & 513.00 & 23.00 & 46.00 & 906.55 \\
\hline 30 & 669 & 576 & 604 & 616.33 & 47.71 & 95.42 & 1089.15 \\
\hline 35 & 797 & 736 & 795 & 776.00 & 34.66 & 69.31 & 1371.31 \\
\hline 40 & 923 & 852 & 903 & 892.67 & 36.61 & 73.22 & 1577.47 \\
\hline 45 & 1004 & 1042 & 1009 & 1018.33 & 20.65 & 41.30 & 1799.54 \\
\hline 50 & 1117 & 1150 & 1165 & 1144.00 & 24.56 & 49.11 & 2021.61 \\
\hline 55 & 1207 & 1233 & 1271 & 1237.00 & 32.19 & 64.37 & 2185.96 \\
\hline 60 & 1414 & 1361 & 1361 & 1378.67 & 30.60 & 61.20 & 2436.31 \\
\hline
\end{tabular}

Instuments Used:

Zephyr-Selomat 12951472, S/N 14714

Cal Exp. Date:

$8 / 12 / 2005$
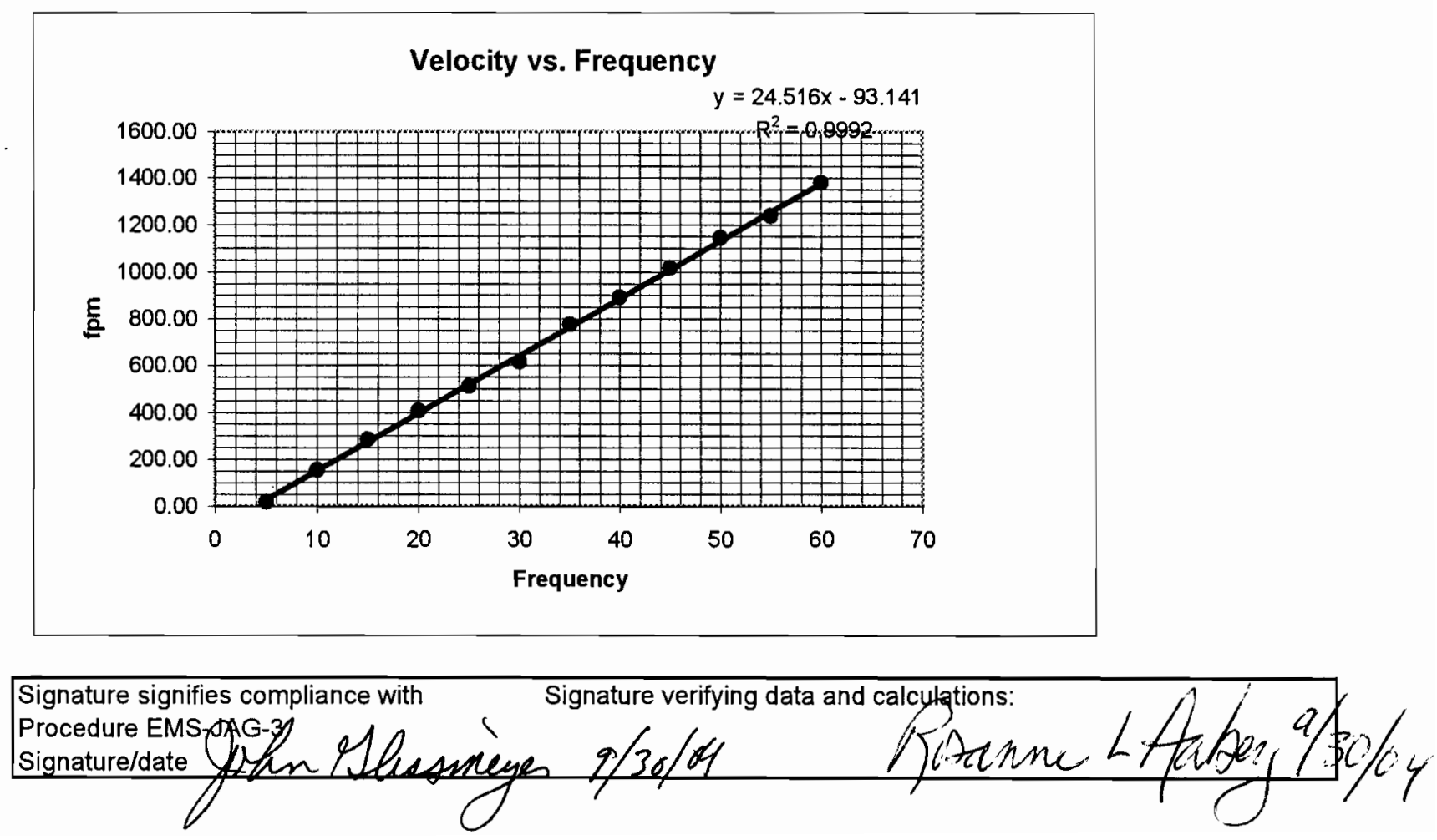


\begin{tabular}{|c|c|c|}
\hline \multicolumn{3}{|c|}{ Test Instruction } \\
\hline $\begin{array}{l}\text { Project: } 325 \text { Stack Sampler } \\
\text { Qualification }\end{array}$ & Date: August 16, 2004 & Work Package: F59676 \\
\hline \multicolumn{3}{|c|}{$\begin{array}{l}\text { Tests: Calibration of Ventilation Flow Controller for } 325 \text { Model Stack, } 1 \text { Electric Fan } \\
\text { Configuration }\end{array}$} \\
\hline \multicolumn{3}{|c|}{ Staff: John Glissmeyer, Dave Douglas, Marcel Ballinger, Matthew Barnett } \\
\hline \multicolumn{3}{|c|}{$\begin{array}{l}\text { Reference Procedures: } \\
\text { 1. Operating Manual for Solomat Zephyr, or other micromanometer used } \\
\text { 2. Procedure EMS-JAG-03 Test to Calibrate Ventilation Flow Controller, Rev. 0, Nov. } \\
\quad 18,1998\end{array}$} \\
\hline \multicolumn{3}{|c|}{$\begin{array}{l}\text { Equipment: } \\
\text { 1. Model Stack, Fans and Fan Speed Controller. Fans will be in positions EF1, EF4. } \\
\text { 2. }\end{array}$} \\
\hline \multicolumn{3}{|c|}{$\begin{array}{l}\text { Safety Considerations: } \\
\text { Review and observe the applicable Job Hazard Analysis for the project }\end{array}$} \\
\hline \multicolumn{3}{|c|}{$\begin{array}{l}\text { Ass: } \\
\text { elevation above the ground. } \\
\text { Measure the stack inside diameter and layout the measurement points for an } 8 \text { point } \\
\text { traverse } \\
\text { With one fan operating, set the flow controller set at midrange }(30 \mathrm{~Hz}) \text { and measure the } \\
\text { velocity at each point. Repeat each measurement thrice. } \\
\text { Record data on velocity data sheets } \\
\text { Identify point of average velocity } \\
\text { Mount pitot tube at that point and measure velocity at } 5 \mathrm{~Hz} \text { increments from } 5 \text { to } 60 \\
\text { Hz. } \\
\text { Record and plot the data } \\
\text { Determine approximate setpoints for subsequent target test flowrate }(1350-1450 \mathrm{cfm} \text {, } \\
764-821 \mathrm{fpm} \text { ) for one operating fan. }(35-37 \mathrm{~Hz}) \\
\text { Diagram mounting fixtures and retain assembly for subsequent tests (duct taje poct })\end{array}$} \\
\hline \multicolumn{3}{|l|}{ Desired Completion Date: $8 / 27 / 04$} \\
\hline \multicolumn{2}{|l|}{ Approvals: Afur \& } & Date $89 / 19 / 04$ \\
\hline \multicolumn{2}{|c|}{ Test completed by: MmT Feant } & Date: $8 / 2 \% / 0 \%$ \\
\hline
\end{tabular}


Appendix C

Velocity Uniformity Procedure and Data 



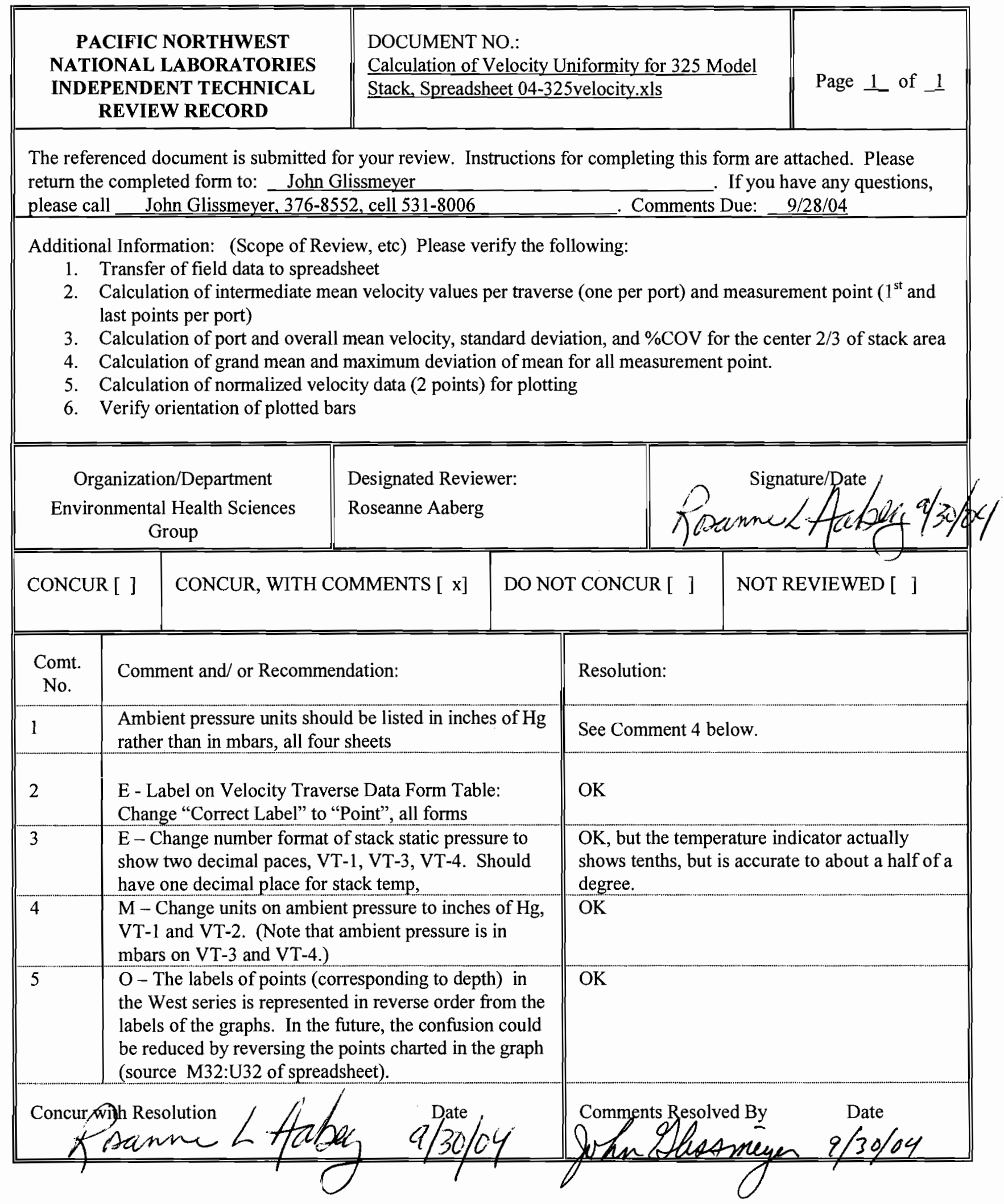


VELOCITY TRAVERSE DATA FORM

Site 325 Model

Date 8/20/2004

Testers MYB/DDD/GSH/JMB

Stack Dia.

Stack X-Area

Elevation

$\frac{18 \text { in. }}{\frac{254.5 \mathrm{in.} 2}{1393 / 4}}$

Distance to disturbance

Velocity units $\underline{\mathrm{tt} / \mathrm{min}}$
Run No. VT-1

Fan Configuration Fan 4 (near stack)

Fan Setting $37.1 \mathrm{~Hz}$

Stack Temp $82.5 \operatorname{deg} F$

Start/End Time 9:07AM - 10:15AM

Center $2 / 3$ from

Points in Center $2 / 3$

Data Files: NA

\begin{tabular}{|c|c|c|c|c|c|c|c|c|c|}
\hline \multirow{2}{*}{\multicolumn{2}{|c|}{$\begin{array}{l}\text { Traverse--> } \\
\text { Trial ---> }\end{array}$}} & \multicolumn{4}{|c|}{ West } & \multicolumn{4}{|c|}{ North } \\
\hline & & 1 & 2 & 3 & Mean & 1 & 2 & 3 & Mean \\
\hline Point & Depth, in. & \multicolumn{4}{|c|}{ Velocity } & \multicolumn{4}{|c|}{ Velocity } \\
\hline 1 & 0.58 & 797 & 810 & 803 & 803.3 & 713 & 781 & 709 & 734.3 \\
\hline 2 & 1.89 & 842 & 874 & 817 & 844.3 & 822 & 803 & 798 & 807.7 \\
\hline 3 & 3.49 & 861 & 855 & 836 & 850.7 & 836 & 835 & 810 & 827.0 \\
\hline 4 & 5.81 & 860 & 857 & 861 & 859.3 & 847 & 810 & 818 & 825.0 \\
\hline Center & 9.00 & 814 & 812 & 838 & 821.3 & 827 & 854 & 837 & 839.3 \\
\hline 5 & 12.19 & 830 & 780 & 769 & 793.0 & 816 & 809 & 803 & 809.3 \\
\hline 6 & 14.51 & 750 & 764 & 793 & 769.0 & 797 & 754 & 808 & 786.3 \\
\hline 7 & 16.11 & 770 & 732 & 741 & 747.7 & 808 & 772 & 781 & 787.0 \\
\hline 8 & 17.42 & 688 & 630 & 628 & 648.7 & 733 & 696 & 610 & 679.7 \\
\hline Averages - & $\longrightarrow$ & 801.3 & 790.4 & 787.3 & 793.0 & 799.9 & 790.4 & 774.9 & 788.4 \\
\hline
\end{tabular}

\begin{tabular}{|lrr|lrrr|}
\hline AII & ft/min & Dev. from mean & Center 2/3 & West & North & All \\
Mean & 790.7 & & Mean & 812.2 & 811.7 & 811.9 \\
Min Point & 648.7 & $-18.0 \%$ & Std. Dev. & 43.2 & 20.2 & 32.4 \\
Max Point & 859.3 & $8.7 \%$ & CoV as \% & 5.3 & 2.5 & 4.0 \\
\hline
\end{tabular}

Flow w/o C-Pt

Vel Avg w/o C-Pt

Stack temp

Equipment temp

Ambient temp

Stack static

Ambient pressure

Total Stack pressure

Ambient humidity
$1389 \mathrm{acfm}$

$786 \mathrm{fpm}$

\begin{tabular}{|c|c|c}
\multicolumn{1}{r|}{ Start } & Finish & \\
\hline 83 & 86 & $F$ \\
\hline 81 & 83 & $F$ \\
\hline 77 & 85 & $F$ \\
\hline 0.02 & 0.02 & mbars \\
\hline 29.5 & 29.5 & in. Hg \\
\hline 1014.0 & 1014.0 & mbars \\
\hline $51 \%$ & $41 \%$ & RH \\
\hline
\end{tabular}

Instuments Used:

$\frac{1.65}{2}$ to: $\frac{16.35}{7}$
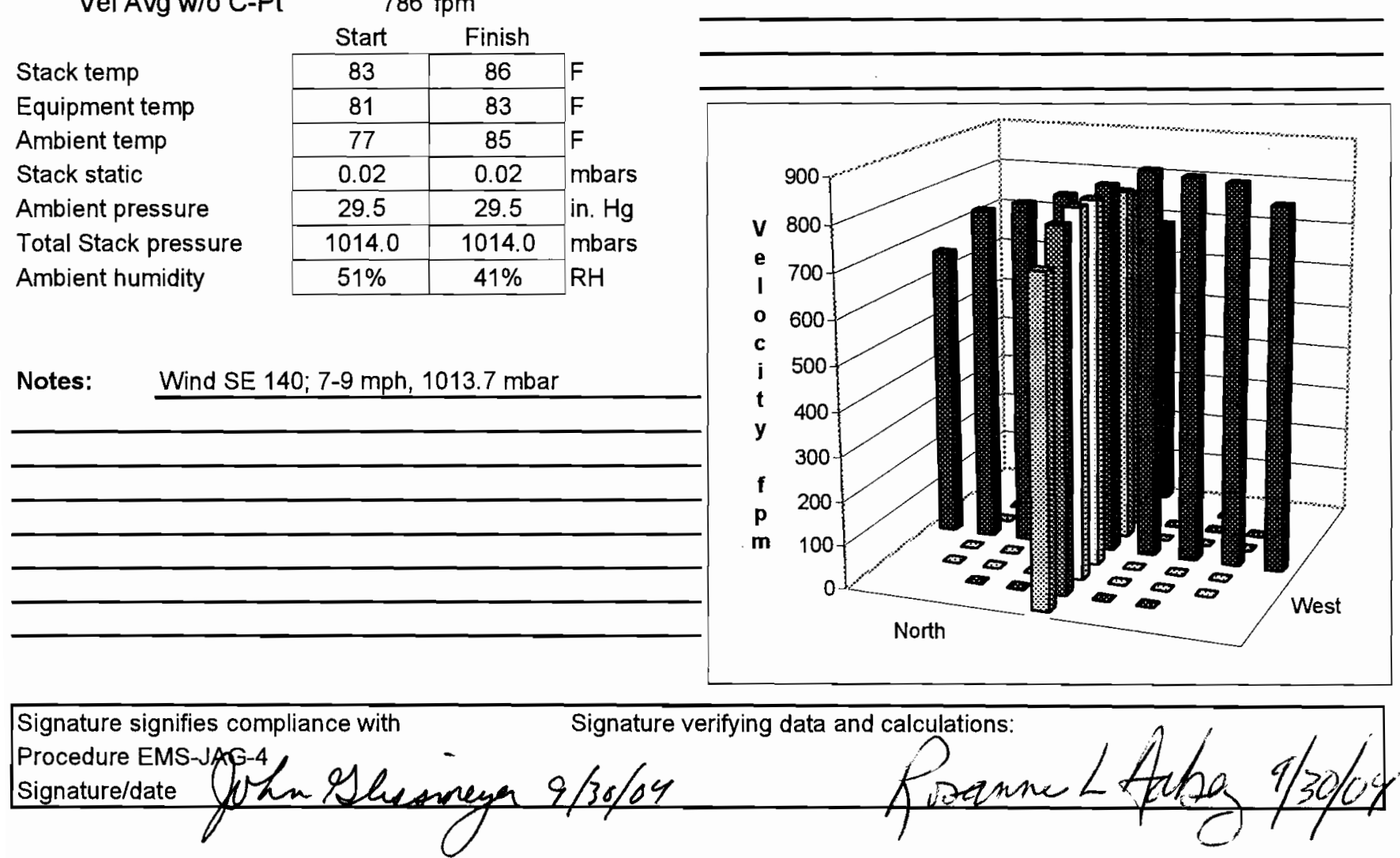


\section{VELOCITY TRAVERSE DATA FORM}

Site 325 Model

Date 8/20/2004

Testers MYB/DDD/GSH/JMB

Stack Dia.

Stack X-Area

Elevation

Distance to disturbance

Velocity units $\mathrm{ft} / \mathrm{min}$
Run No. VT-2

Fan Configuration Fan 1 (far from stack)

Fan Setting $37.1 \mathrm{~Hz}$

Stack Temp $85.6 \operatorname{deg} F$

Start/End Time 10:25-11:32

Center $2 / 3$ from 1.65 to: 16.35

Points in Center $2 / 3 \overline{2}$ to: $\overline{7}$

Data Files: NA

\begin{tabular}{|c|c|c|c|c|c|c|c|c|c|}
\hline \multirow{2}{*}{\multicolumn{2}{|c|}{$\begin{array}{l}\text { Traverse--> } \\
\text { Trial -_-> }\end{array}$}} & \multicolumn{4}{|c|}{ West } & \multicolumn{4}{|c|}{ North } \\
\hline & & 1 & 2 & 3 & Mean & 1 & 2 & 3 & Mean \\
\hline Point & Depth, in. & \multicolumn{4}{|c|}{ Velocity } & \multicolumn{4}{|c|}{ Velocity } \\
\hline 1 & 0.58 & 742 & 745 & 694 & 727.0 & 750 & 660 & 696 & 702.0 \\
\hline 2 & 1.89 & 791 & 786 & 762 & 779.7 & 753 & 760 & 726 & 746.3 \\
\hline 3 & 3.49 & 767 & 775 & 780 & 774.0 & 769 & 769 & 736 & 758.0 \\
\hline 4 & 5.81 & 772 & 788 & 760 & 773.3 & 734 & 786 & 797 & 772.3 \\
\hline Center & 9.00 & 760 & 778 & 760 & 766.0 & 734 & 771 & 757 & 754.0 \\
\hline 5 & 12.19 & 781 & 792 & 801 & 791.3 & 757 & 761 & 768 & 762.0 \\
\hline 6 & 14.51 & 778 & 787 & 785 & 783.3 & 801 & 769 & 757 & 775.7 \\
\hline 7 & 16.11 & 766 & 767 & 801 & 778.0 & 747 & 782 & 742 & 757.0 \\
\hline 8 & 17.42 & 611 & 699 & 679 & 663.0 & 769 & 716 & 712 & 732.3 \\
\hline Averages & $\longrightarrow$ & 752.0 & 768.6 & 758.0 & 759.5 & 757.1 & 752.7 & 743.4 & 751.1 \\
\hline
\end{tabular}

\begin{tabular}{|c|c|c|c|c|c|c|}
\hline$A I I$ & $\underline{\mathrm{ft} / \mathrm{min}}$ & Dev. from mean & Center $2 / 3$ & West & North & All \\
\hline Mean & 755.3 & & Mean & 778.0 & 760.8 & 769.4 \\
\hline Min Point & 663.0 & $-12.2 \%$ & Std. Dev. & 8.1 & 10.3 & 12.6 \\
\hline Max Point & 791.3 & $4.8 \%$ & COV as $\%$ & 1.0 & 1.4 & 1.6 \\
\hline
\end{tabular}

Flow w/o C-P Vel Avg w/o C-Pt

Stack temp

Equipment temp Ambient temp

Stack static

Ambient pressure

Total Stack pressure

Ambient humidity

Notes: $\quad$ wind from SW 6-9 mph
Instuments Used:

Solomat Zephyr \#14714

\begin{tabular}{|c|c|}
\hline Start & Finish \\
\hline 86 & 91 \\
\hline 83 & 84 \\
\hline 85 & 90 \\
\hline 0.02 & 0.02 \\
\hline 29.5 & 29.5 \\
\hline 1014.0 & 1013.0 \\
\hline $41 \%$ & $31 \%$ \\
\hline
\end{tabular}

(2)
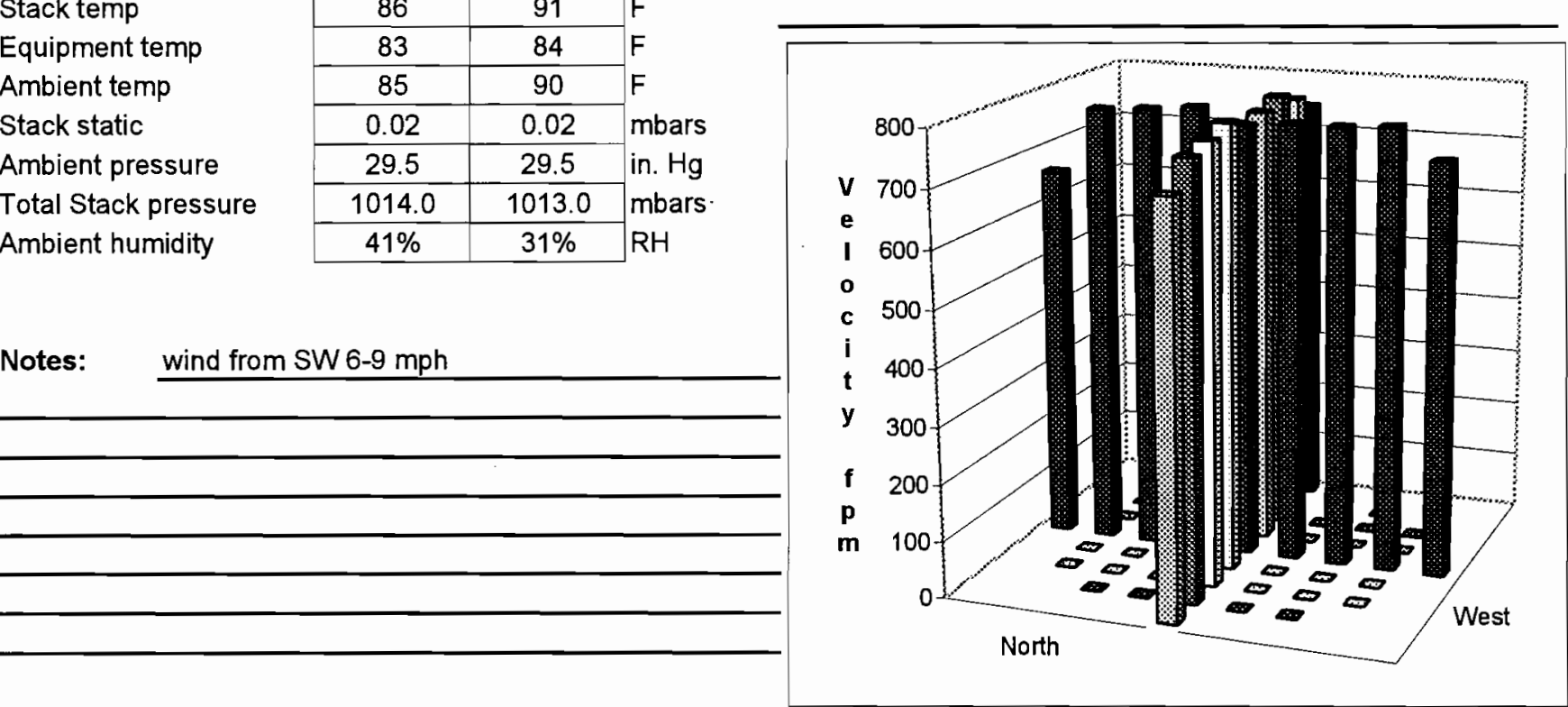

Signature signifies compliance with

Procedure EMS-JAG-4 Yhature/date OHherenen $9 / 30 / 04$ Signature verifying data and calcylations:

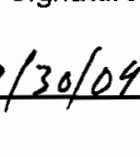

$+x=2 n n$

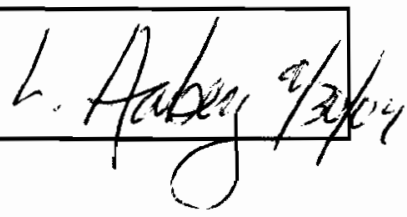


VELOCIIY TRAVERSE DATA FORM

Site 325 Model

Date 8/26/2004

Testers JAG/GSH/JMB

Stack Dia.

Stack X-Area

Elevation

Distance to disturbance

Velocity units $\underline{\mathrm{ft} / \mathrm{min}}$
Run No. VT-3

Fan Configuration Near Fan (\#4)

Fan Setting $37.1 \mathrm{~Hz}$

Stack Temp $82.4 \operatorname{deg} \mathrm{F}$

Start/End Time 11:22

Center $2 / 3$ from

Points in Center $2 / 3$

Data Files: NA

\begin{tabular}{|c|c|c|c|c|c|c|c|c|c|}
\hline \multirow{2}{*}{$\begin{array}{l}\text { Traverse--> } \\
\text { Trial } \longrightarrow>\end{array}$} & & \multicolumn{4}{|c|}{ West } & \multicolumn{4}{|c|}{ North } \\
\hline & & 1 & 2 & 3 & Mean & 1 & 2 & 3 & Mean \\
\hline Point & Depth, in. & \multicolumn{4}{|c|}{ Velocity } & \multicolumn{4}{|c|}{ Velocity } \\
\hline 1 & 0.58 & 827 & 831 & 771 & 809.7 & 772 & 700 & 742 & 738.0 \\
\hline 2 & 1.89 & 830 & 888 & 819 & 845.7 & 829 & 784 & 784 & 799.0 \\
\hline 3 & 3.49 & 858 & 896 & 844 & 866.0 & 836 & 817 & 843 & 832.0 \\
\hline 4 & 5.81 & 918 & 894 & 835 & 882.3 & 855 & 832 & 856 & 847.7 \\
\hline Center & 9.00 & 851 & 821 & 827 & 833.0 & 841 & 856 & 828 & 841.7 \\
\hline 5 & 12.19 & 800 & 837 & 781 & 806.0 & 821 & 839 & 801 & 820.3 \\
\hline 6 & 14.51 & 859 & 798 & 740 & 799.0 & 821 & 851 & 804 & 825.3 \\
\hline 7 & 16.11 & 803 & 793 & 766 & 787.3 & 785 & 811 & 803 & 799.7 \\
\hline 8 & 17.42 & 751 & 633 & 671 & 685.0 & 694 & 784 & 697 & 725.0 \\
\hline Averages - & $\rightarrow>$ & 833.0 & 821.2 & 783.8 & 812.7 & 806.0 & 808.2 & 795.3 & 803.2 \\
\hline
\end{tabular}

\begin{tabular}{|lrr|lrrr|}
\hline All & ft/min & Dev. from mean & Center 2/3 & $\underline{\text { West }}$ & $\underline{\text { North }}$ & All \\
\cline { 2 - 6 } & 807.9 & & Mean & 831.3 & 823.7 & 827.5 \\
Min Point & 685.0 & $-15.2 \%$ & Std. Dev. & 35.7 & 19.0 & 27.7 \\
Max Point & 882.3 & $9.2 \%$ & Cov as $\%$ & 4.3 & 2.3 & 3.4 \\
\hline
\end{tabular}

Flow w/o C-Pt

Vel Avg w/o C-Pt

Stack temp

Equipment temp

Ambient temp

Stack static

Ambient pressure

Total Stack pressure

Ambient humidity
$1421 \mathrm{acfm}$

$804 \mathrm{fpm}$

\begin{tabular}{|c|c|l|}
\multicolumn{1}{c|}{ Start } & Finish & \\
\hline 81 & 84 & $\mathrm{~F}$ \\
\hline 74 & 79 & $\mathrm{~F}$ \\
\hline 74 & 76 & $\mathrm{~F}$ \\
\hline 0.02 & 0.00 & mbars \\
\hline 1016.5 & 1016.5 & mbars \\
\hline 1017.0 & 1017.0 & mbars \\
\hline $43 \%$ & $38 \%$ & $\mathrm{RH}$ \\
\hline
\end{tabular}

Notes:

Instuments Used:

Solomat Zephyr SN 1295-1472
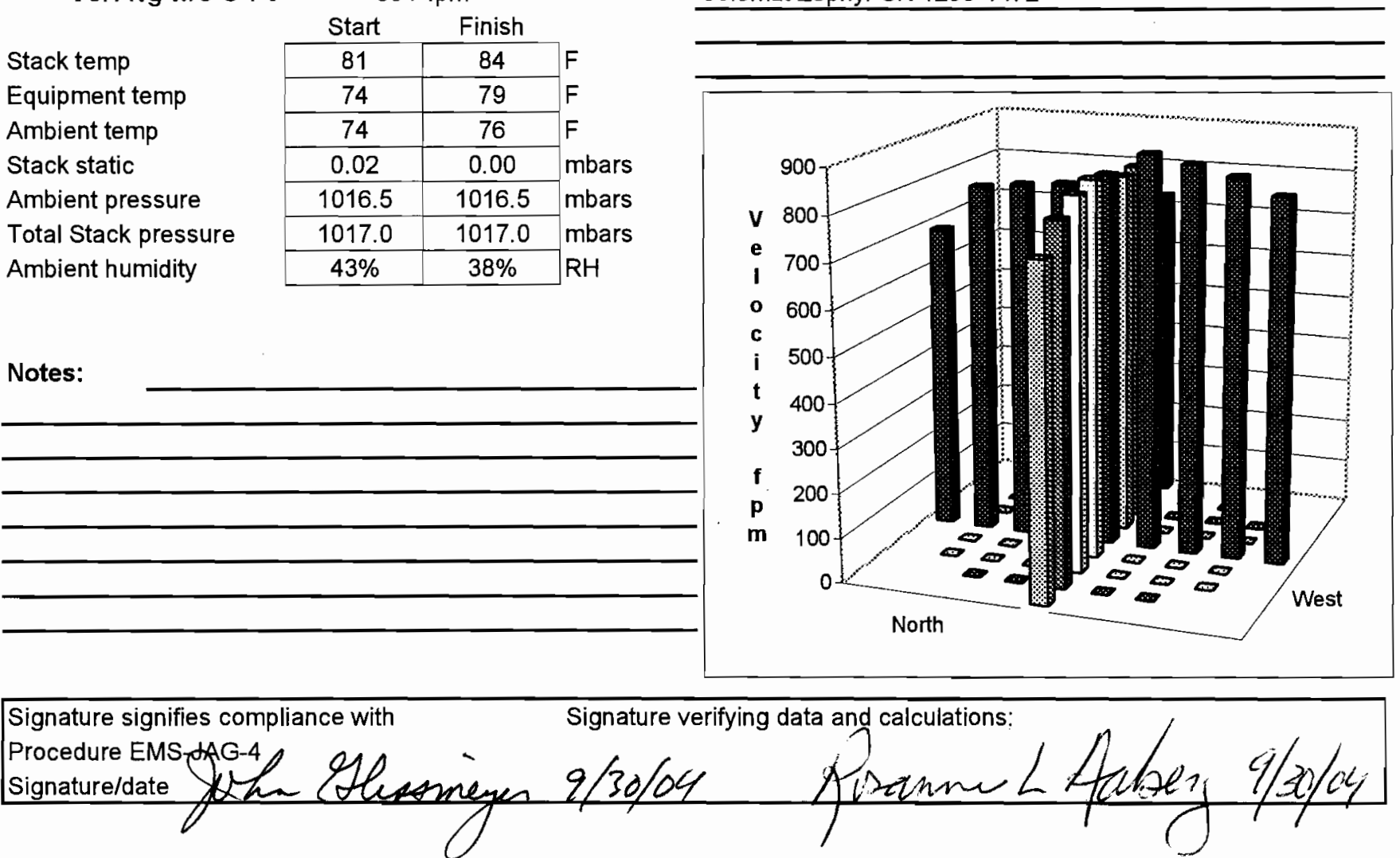
VELOCITY TRAVERSE DATA FORM

Site 325 Model

Date 8/26/2004

Testers JAG/GSH

Stack Dia

Stack X-Area

Elevation

Distance to disturbance

Velocity units $\mathrm{ft} / \mathrm{min}$
Run No. VT-4

Fan Configuration Far Fan (\#1)

Fan Setting $37.1 \mathrm{~Hz}$

Stack Temp 86.9 deg $F$

Start/End Time 13:21

Center 2/3 from

Points in Center $2 / 3$

Data Files: $\mathrm{NA}$

\begin{tabular}{|c|c|c|c|c|c|c|c|c|c|}
\hline \multirow{2}{*}{$\begin{array}{l}\text { Traverse--> } \\
\text { Trial ---> }\end{array}$} & & \multicolumn{4}{|c|}{ West } & \multicolumn{4}{|c|}{ North } \\
\hline & & 1 & 2 & 3 & Mean & 1 & 2 & 3 & Mean \\
\hline Point & Depth, in. & \multicolumn{4}{|c|}{ Velocity } & \multicolumn{4}{|c|}{ Velocity } \\
\hline 1 & 0.58 & 739 & 719 & 743 & 733.7 & 682 & 604 & 717 & 667.7 \\
\hline 2 & 1.89 & 751 & 782 & 782 & 771.7 & 767 & 763 & 789 & 773.0 \\
\hline 3 & 3.49 & 768 & 779 & 813 & 786.7 & 810 & 788 & 772 & 790.0 \\
\hline 4 & 5.81 & 716 & 789 & 782 & 762.3 & 807 & 764 & 791 & 787.3 \\
\hline Center & 9.00 & 755 & 741 & 774 & 756.7 & 771 & 760 & 767 & 766.0 \\
\hline 5 & 12.19 & 762 & 790 & 779 & 777.0 & 760 & 782 & 797 & 779.7 \\
\hline 6 & 14.51 & 786 & 797 & 780 & 787.7 & 796 & 796 & 742 & 778.0 \\
\hline 7 & 16.11 & 769 & 745 & 693 & 735.7 & 789 & 760 & 784 & 777.7 \\
\hline 8 & 17.42 & 637 & 677 & 623 & 645.7 & 738 & 726 & 750 & 738.0 \\
\hline Averages - & 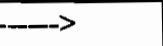 & 742.6 & 757.7 & 752.1 & 750.8 & 768.9 & 749.2 & 767.7 & 761.9 \\
\hline
\end{tabular}

\begin{tabular}{|c|c|c|c|c|c|c|}
\hline$A \|$ & $\underline{\mathrm{ft} / \mathrm{min}}$ & Dev. from mean & Center 2/3 & West & North & All \\
\hline Mean & 756.4 & & Mean & 768.2 & 778.8 & 773.5 \\
\hline Min Point & 645.7 & $-14.6 \%$ & Std. Dev. & 18.4 & 8.1 & 14.7 \\
\hline Max Point & 790.0 & $4.4 \%$ & COV as $\%$ & 2.4 & 1.0 & 1.9 \\
\hline
\end{tabular}

Flow w/o C-Pt 1335 acfm

Vel Avg w/o C-Pt $\quad 756 \mathrm{fpm}$

Stack temp

Equipment temp

Ambient temp

Stack static

Ambient pressure

Total Stack pressure

Ambient humidity

\begin{tabular}{|c|c|}
\hline Start & Finish \\
\hline 85 & 89 \\
\hline 79 & 80 \\
\hline 80 & 80 \\
\hline 0.01 & -0.04 \\
\hline 1016.4 & 1016.8 \\
\hline 1016.4 & 1016.8 \\
\hline $28 \%$ & $28 \%$ \\
\hline
\end{tabular}

Instuments Used:

Solomat Zephyr SN 1295-1472

Notes:
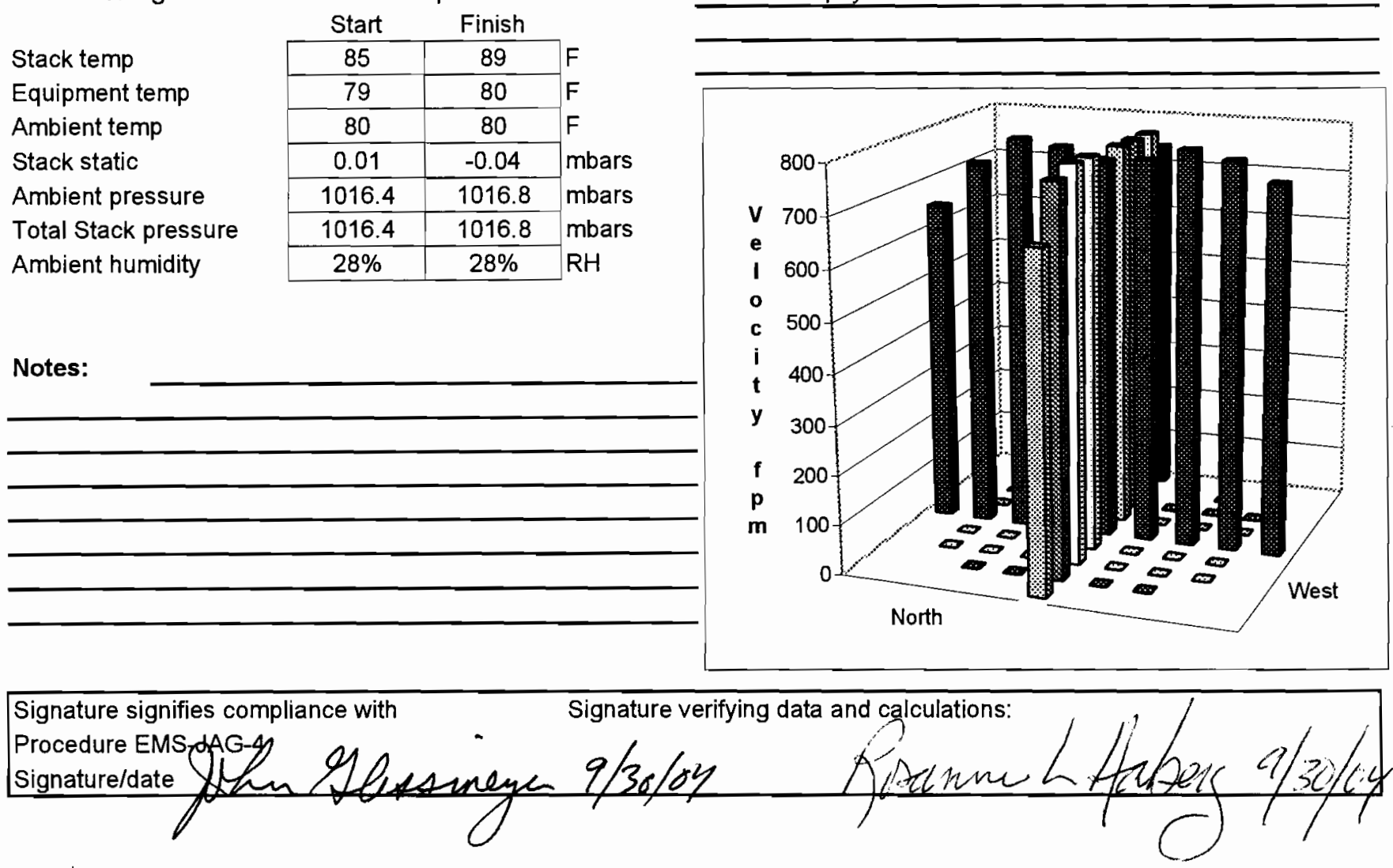


\section{Test Instruction}

\begin{tabular}{l|l|l}
\hline $\begin{array}{l}\text { Project: } 325 \text { Stack Sampler } \\
\text { Qualification }\end{array}$ & Date: August 16, 2004 & Work Package: F59676 \\
\hline
\end{tabular}

Tests: Velocity Uniformity in 325 Model Stack, 1 Electric Fan Configuration

Staff: John Glissmeyer, Dave Douglas, Marcel Ballinger, Matthew Barnett

Reference Procedures:

1. Operating Manual for air velocity instrument used

2. Procedure EMS-JAG-04, Test to Determine Uniformity of Gas Velocity at the Elevation of a Sampler Probe

3. Model Stack, Fans and Fan Speed Controller. Fans will be in positions EF1, EF4.

Equipment:

1. TSI VelociCalc, Solomat Zephyr and 36" standard pitot tube, or other calibrated velocity measurement instrumentation

Safety Considerations:

Observe the applicable Job Hazard Analysis for the project

Instructions:

1. Verify training on the procedure and that instrumentation is within calibration.

2. Assemble the equipment at the ports at the 12.5 foot elevation above the ground. Use the same measurement points as for flow calibration test.

3. Mark the completion of each step on the field copy of the procedure. Mark-out those steps not applicable to this stack.

4. With one operating fan, set the fan controller to achieve the desired approximate flowrate $(1350$ - $1450 \mathrm{cfm})$

5. Record data on velocity data sheet

6. If target flowrate is not achieved, adjust fan controller and rerun test.

7. Repeat this test with the other fan operating.

8. Repeat this test again with either fan operating or with the configuration yielding the highest \% COV from the first two tests.

9. Diagram mounting fixtures and retain assembly for subsequent tests

Desired Completion Date: 08/27/04
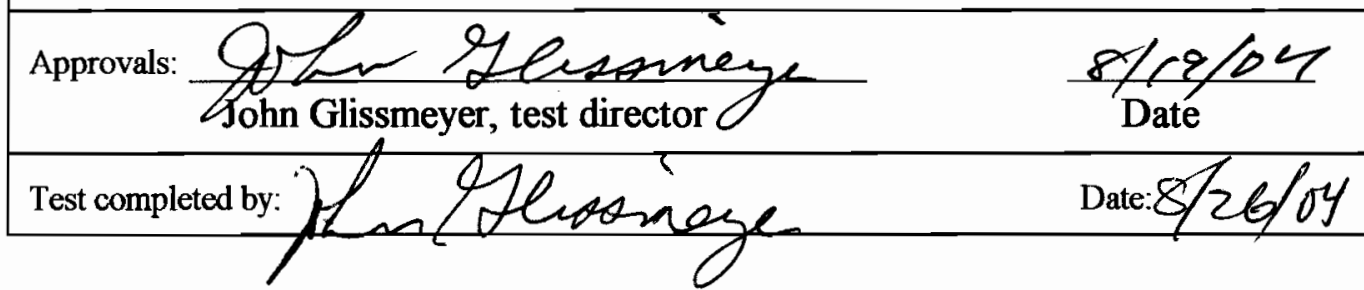


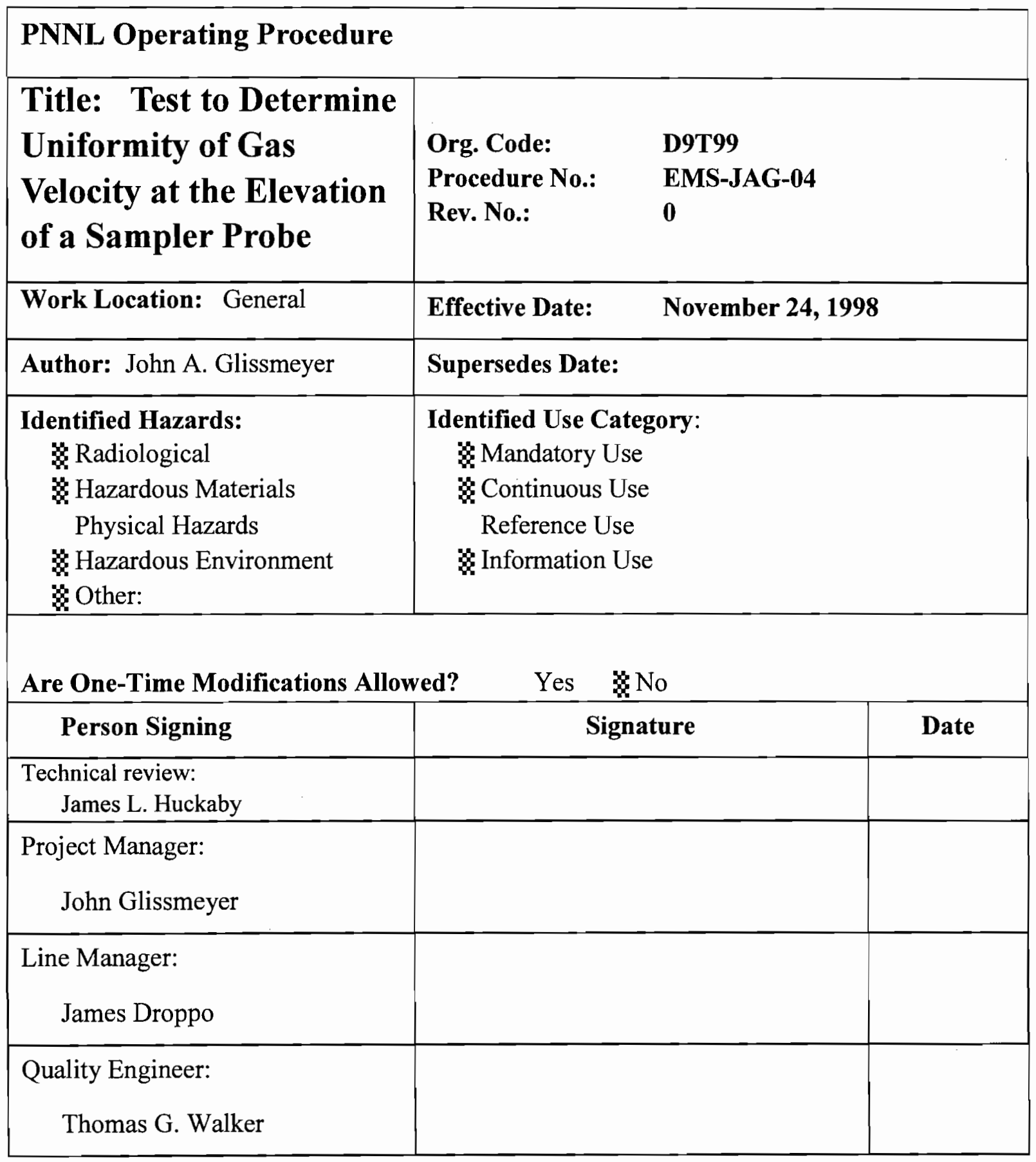


Title: Test to Determine Uniformity of Gas Velocity at the Elevation of a Sampler Probe

\subsection{Purpose}

The performance of new stack sampling systems must be shown to satisfy the requirements of 40 CFR 61, Subpart H, "National Emission standards for Emissions of Radionuclides Other than Radon from Department of Energy Facilities." This regulation governs portions of the design and implementation of effluent air sampling. The stack sampler performance is adequately characterized when potential contaminants in the effluent are of a uniform concentration at the sampling plane and line losses are within acceptable limits. (The sampling plane is the cross section of the stack or duct where the sampling nozzle inlet is located.) Uniformity of contaminant concentration is unlikely where the gas velocity throughout the sampling plane is significantly non-uniform. This procedure provides the means to determine the uniformity of gas velocity, and is performed prior to measurements of contaminant uniformity. This procedure is performed after the range of gas flow conditions are established. Other procedures that usually follow address flow angle, and uniformity of gas and aerosol contaminants.

\subsection{Applicability}

This procedure can be used in the field or on modeled stacks and ducts to determine the uniformity of air velocity throughout the sampling plane. The results also provide a detailed determination of the flowrate at the ventilation control settings used for the procedure. The tests are applicable within the following constraints:

- The operating limits of the air velocity measurement device used are observed.

- The air velocity sensor element does not occupy more than a few percent of the cross sectional area in the sampling plane.

This procedure may need to be repeated if there are changes made in the configuration of the ventilation system. If the system under test operates within a limited range of airflow that does not change more than $\pm 25 \%$, then this procedure is usually conducted once at the middle of the range. If the flow may vary more, then the procedure is performed at least at the extremes of flow.

\subsection{Prerequisites and Conditions}

Conditions and concerns that must be satisfied prior to performing this procedure are listed below: 


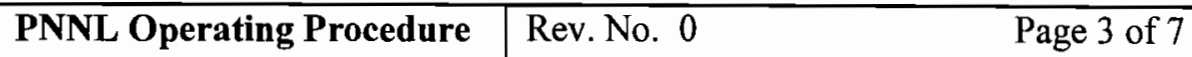

Org. Code: D9T99 Procedure No.: EMS-JAG-04

Title: Test to Determine Uniformity of Gas Velocity at the Elevation of a Sampler Probe

- The job-hazards analysis for the work area must be prepared and followed.

- Safety glasses, hard toed or substantial shoes may be required in the work areas.

- Scaffold user training may be required to access the sampling ports of the stack.

- The flow ventilation control device must be installed and means available for its adjustment.

- Air velocity measurement equipment must be within calibration.

- The test instruction must be read and understood.

\subsection{Precautions and Limitations}

Access to the test ports may require the use of ladders, scaffolding or manlifts, which may necessitate special training for sampling personnel and any observers. The training requirements will be indicated in the job hazard analysis.

\subsection{Equipment Used for Measurements}

The following are essential items of equipment:

- Air velocity measurement apparatus, which may consist of a calibrated slant tube or electronic manometer, pitot tube, or some other type of sensor;

- Platform, ladders, or manlifts as needed to access the test ports;

- Fittings to limit leakage around the velocity sensor and to stabilize the sensor so it can be repositioned repeatably.

Further details on specific equipment for the job are provided in the Test Instruction. The air velocity instrumentation may be either the types used in 40 CFR 60, Appendix A, Method 2, or other measurement device for discrete points, such as a rotating vane or thermal anemometer. The user must be aware that different devices may give readings in terms of different gas conditions.

\subsection{Work Instructions for Setup, Measurements, and Data Reduction}

Job specific instructions given in the Test Instruction, illustrated in Exhibit B, will provide details and operating parameters necessary to perform this procedure. 
\begin{tabular}{l|ll} 
PNNL Operating Procedure & Rev. No. 0 & Page 4 of 7
\end{tabular}

Org. Code: D9T99 Procedure No.: EMS-JAG-04

Title: Test to Determine Uniformity of Gas Velocity at the Elevation of a Sampler Probe

\subsection{Preliminary Steps:}

6.1.1 Verify that the interior dimensions of the stack or duct at the sampling plane agree with those used in calculating the grid of measurement points given in the test instruction or data sheet.

6.1.2 Provide essential supplies at the sampling location (velocity measuring instrumentation, fittings to adapt the sensor to the test ports, marking pens, data sheets, writing and sensor supporting platforms).

6.1.3 Verify that the ventilation flow control device is capable of the flow control settings given in the Test Instruction.

$\checkmark$ 6.1.4 Prepare a data sheet for the detailed velocity traverse. See illustration in Exhibit A. Label the columns of traverse data by the direction of the traverse.

Note. For example, if the first reading is closest to the east port, and the last reading is closest to the west port, then label the traverse east-west. Also the first point is the one closest to the port.

Note. The grid of velocity measurement points is calculated in accordance with 40 CFR 60, Appendix A, Method 1. A centerpoint is included as a common reference and for graphical purposes. The layout design divides the area of the sampling plane so that each point represents approximately an equal-sized area

6.1.5 Mark the velocity sensor body to indicate the insertion depth for each point in the measurement grid.

6.1.6 Obtain barometric pressure, relative humidity, and stack or duct temperature and static pressure if needed to convert the velocity sensor readings to velocity units.

6.1.7 Insert the velocity sensor in the stack or duct and seal the opening around it. 
Title: Test to Determine Uniformity of Gas Velocity at the Elevation of a Sampler Probe

\subsection{Velocity Uniformity Measurement}

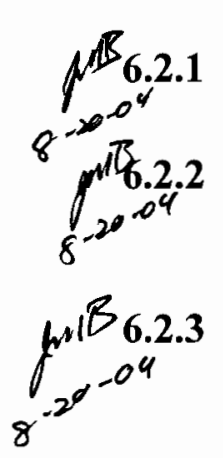

Set the flow controller per the test instruction.

Verify that the directional orientations and the numbered measurement positions are consistent with the data sheet.

Measure and record, on the data sheet, the velocity or pressure reading at each measurement point in succession. If the readout device has an averaging feature, record the average of a series of several readings. -

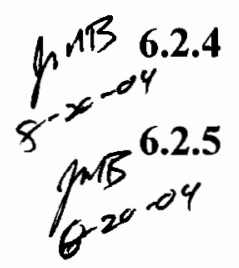

Repeat Step 6.2.3.

Compare the results in Step 6.2.3 with those of 6.2.4. If the measurements are not highly reproducible, repeat Step 6.2.3 again.

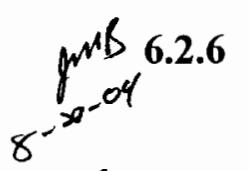

Calculate the average air velocity for each measurement point.

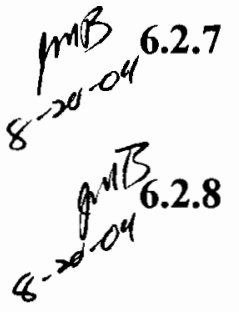

Calculate the overall average velocity and flowrate for the stack or duct, omitting the center point.

6.2.8 Calculate the coefficient of variance (COV, 100 times the standard deviation divided by the mean) using the average velocity for all points in the inner two-thirds of the cross section area (including the centerpoint).

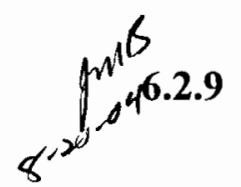

Compare the observed COV for each run to the acceptance criterion. The acceptance criterion for the COV is $\# 20 \%$ for the inner two-thirds of the stack diameter.

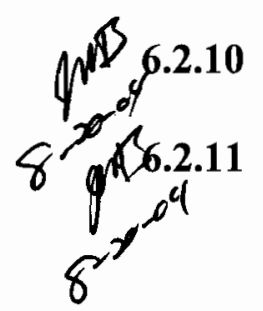




\begin{tabular}{|l|ll|}
\hline PNNL Operating Procedure & $\begin{array}{l}\text { Rev. No. } 0 \\
\text { Org. Code: D9T99 }\end{array}$ & $\begin{array}{l}\text { Page } 6 \text { of } 7 \\
\text { Procedure No.: EMS-JAG-04 }\end{array}$ \\
\hline \multicolumn{2}{|c|}{ Title: Test to Determine Uniformity of Gas Velocity at the Elevation of a Sampler Probe } \\
\hline
\end{tabular}

\subsection{Exhibits/Attachments}

\section{Exhibit A - Illustration of Detailed Velocity Traverse Data Sheet}

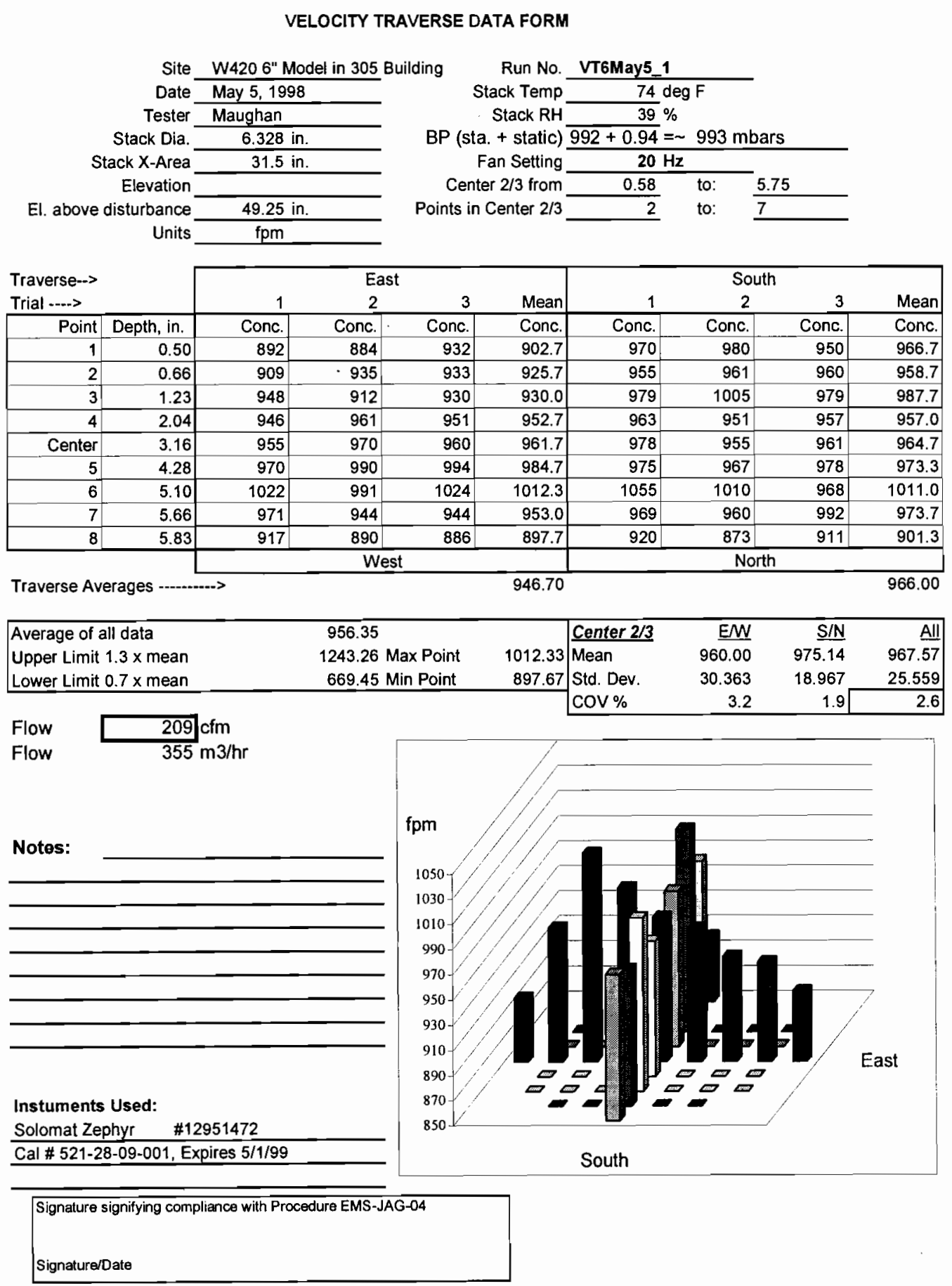




\begin{tabular}{|l|ll|}
\hline PNNL Operating Procedure & $\begin{array}{l}\text { Rev. No. 0 } \\
\text { Org. Code: D9T99 }\end{array}$ & $\begin{array}{l}\text { Page } 7 \text { of } 7 \\
\text { Procedure No.: EMS-JAG-04 }\end{array}$ \\
\hline \multicolumn{2}{|c|}{ Title: Test to Determine Uniformity of Gas Velocity at the Elevation of a Sampler Probe } \\
\hline
\end{tabular}

\section{Exhibit B - Illustrative Test Instruction}

\begin{tabular}{|c|c|c|}
\hline \multicolumn{3}{|c|}{ Test Instruction } \\
\hline $\begin{array}{l}\text { Project: W420 6" Stack } \\
\text { Calibration } 28361\end{array}$ & Date: August 19, 1998 & Work Package: K83017 \\
\hline \multicolumn{3}{|c|}{ Tests: Velocity Uniformity High Flow in W420 6" Full-Scale Model Stack } \\
\hline \multicolumn{3}{|l|}{ Staff: David Maughan } \\
\hline \multicolumn{3}{|c|}{$\begin{array}{l}\text { Reference Procedures: } \\
\text { 1. Operating Manual for Solomat Zephyr } \\
\text { 2. Test to Determine Uniformity of Gas Velocity at the Elevation of a Sampler Probe, } \\
\text { Procedure EMS-JAG-04 }\end{array}$} \\
\hline \multicolumn{3}{|c|}{$\begin{array}{l}\text { Equipment: } \\
\text { 1. W420 6" Full-Scale Model Stack, Fan and Fan Speed Controller located in } 305 \text { Bldg. } \\
\text { 2. Solomat Zephyr and pitot tube }\end{array}$} \\
\hline \multicolumn{3}{|c|}{$\begin{array}{l}\text { Safety Considerations: } \\
\text { Review and observe the applicable Numatec Job Hazard Analysis for the project }\end{array}$} \\
\hline \multicolumn{3}{|c|}{$\begin{array}{l}\text { Instructions: } \\
\text { 1. Assemble the equipment for the velocity uniformity test at the ports at the elevation of the } \\
\text { sampling probe } \\
\text { 2. Layout the measurement points with the following distances from the inside of the stack } \\
\text { wall: } 0.5,0.66,1.23,2.04,3.16,4.28,5.10,5.66,5.83 \text { inches. } 3 \text {. Measure the velocity at } \\
\text { each point at the high }(400 \mathrm{cfm}) \text { extreme of stack flow. Repeat each measurement twice. } \\
\text { 4. Record data on velocity data sheets } \\
\text { 5. Diagram mounting fixtures and retain assembly for subsequent tests }\end{array}$} \\
\hline \multicolumn{3}{|c|}{ Desired Completion Date: $12 / 5 / 98$} \\
\hline Approvals: & & \\
\hline & project manager & Date \\
\hline
\end{tabular}



Appendix D

Flow Angle Procedure and Data 

INDEPENDENT TECHNICAL REVIEW RECORD

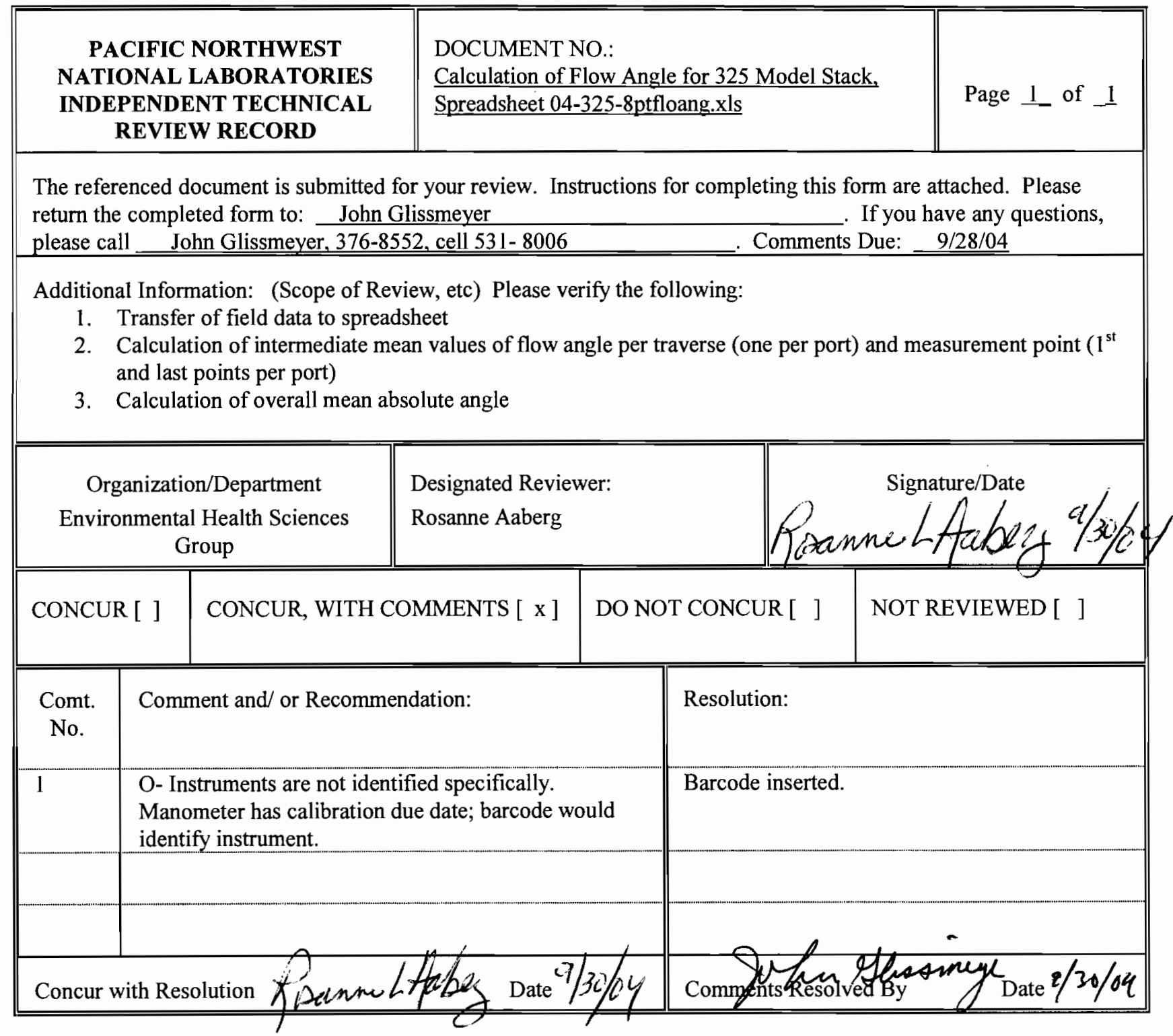




\begin{tabular}{|c|c|c|}
\hline Site & 5 Mode & \\
\hline Date & $20 / 2004$ & \\
\hline Tester & YB/GSH & $\mathrm{MB}$ \\
\hline Stack Dia. & 18 & in \\
\hline Stack X-Area & 254.5 & in2 \\
\hline Elevation & 121.5 & in \\
\hline Distance to disturbance & 75 & in \\
\hline
\end{tabular}

Site 325 Model

Date 8/20/2004

Tester MYB/GSH/JMB

Traverse-->
Trial --->
\begin{tabular}{|c|r|}
\hline Point & Depth, in. \\
\hline 1 & 0.58 \\
\hline 2 & 1.89 \\
\hline 3 & 3.49 \\
\hline 4 & 5.81 \\
\hline Center & 9.00 \\
\hline 5 & 12.19 \\
\hline 6 & 14.51 \\
\hline 7 & 16.11 \\
\hline 8 & 17.42 \\
\hline
\end{tabular}

Mean of absolute values w/o points by wall:

Instuments Used:

S-type pitot

Stanley protractor level

Manometer

Pitot 2
Traverse-->

\begin{tabular}{|c|c|}
\hline 1 & 2 \\
\hline deg. cw & deg. cw \\
\hline-15 & -8 \\
\hline-14 & -7 \\
\hline-4 & -8 \\
\hline-7 & -7 \\
\hline 3 & -1 \\
\hline 6 & 5 \\
\hline 7 & 9 \\
\hline 9 & 12 \\
\hline 10 & 12 \\
\hline 8.3 & 7.7 \\
\hline 7.1 & 7.0 \\
\hline
\end{tabular}

East

3

deg. Cw

-15
-20

$-20$

$-15$

$\frac{-9}{-1}$

$-1 \quad 0.3$

$1 \quad 4.0$

12

10

9

10.2

9.7

\begin{tabular}{|c|c|}
\hline Avg \\
\hline-12.7 \\
\hline-13.7 \\
\hline-9.0 \\
\hline-7.7 \\
\hline 0.3 \\
\hline 4.0 \\
\hline 9.3 \\
\hline 10.3 \\
\hline 10.3 \\
\hline
\end{tabular}

\begin{tabular}{|c|c} 
& 1 \\
\hline Avg. & deg. cw \\
\hline 12.7 & -13 \\
\hline 13.7 & -12 \\
\hline 9.0 & -10 \\
\hline 7.7 & -4 \\
\hline 0.3 & 3 \\
\hline 4.0 & 7 \\
\hline 9.3 & 11 \\
\hline 0.3 & 12 \\
\hline 0.3 & 14 \\
& 9.6 \\
& 8.4
\end{tabular}

\begin{tabular}{|c|c|}
\hline 2 & 2 \\
\hline deg. \\
\hline-14 \\
-13 \\
\hline-10 \\
\hline-8 \\
\hline-5 \\
\hline 5 \\
\hline 10 \\
\hline 12 \\
\hline 16 \\
\hline 10.3 \\
9.0 \\
\hline
\end{tabular}

Notes:

To assure similar hose connections

between the manometer and pitot tube, rotating the pitot tube assembly clockwise drives the meniscus to the right (to higher pos. numbers).
Fan Setting $\frac{37.1 \mathrm{~Hz}}{37}$

Fan configuration Fan 1 (Far from stack)

Approx. stack flow $1442 \quad \mathrm{cfm}$

Units degrees (clockwise > pos. nos.)

\section{Manometer Cal. Due}

\section{North}

$2 \quad 3$

\begin{tabular}{l|c|c}
\hline . CW & deg. CW & Avg \\
\hline-14 & -17 & -14.7 \\
\hline
\end{tabular}

$\begin{array}{lll}-13 & -16 & -13.7\end{array}$

\begin{tabular}{l|l|l}
10 & -12 & -10.7
\end{tabular}

\begin{tabular}{l|l|l}
-8 & -6 & -6.0
\end{tabular}

\begin{tabular}{l|l|l}
\hline-5 & 4 & -6.0 \\
\hline 5 & 0.7
\end{tabular}

\begin{tabular}{l|l|l}
5 & 7 & 6.3 \\
\hline
\end{tabular}

\begin{tabular}{l|l|l}
\hline 10 & 11 & 10.7 \\
\hline
\end{tabular}

\begin{tabular}{l|l|l}
12 & 12 & 12.0 \\
\hline
\end{tabular}

\begin{tabular}{l|l|l}
16 & 15 & 15.0 \\
\hline
\end{tabular}

$3 \quad 11.1$

$\begin{array}{ll}9.3 & 9.7\end{array}$

\begin{tabular}{rr} 
all & 9.5 \\
w/o wall pts & 8.5 \\
\hline
\end{tabular}

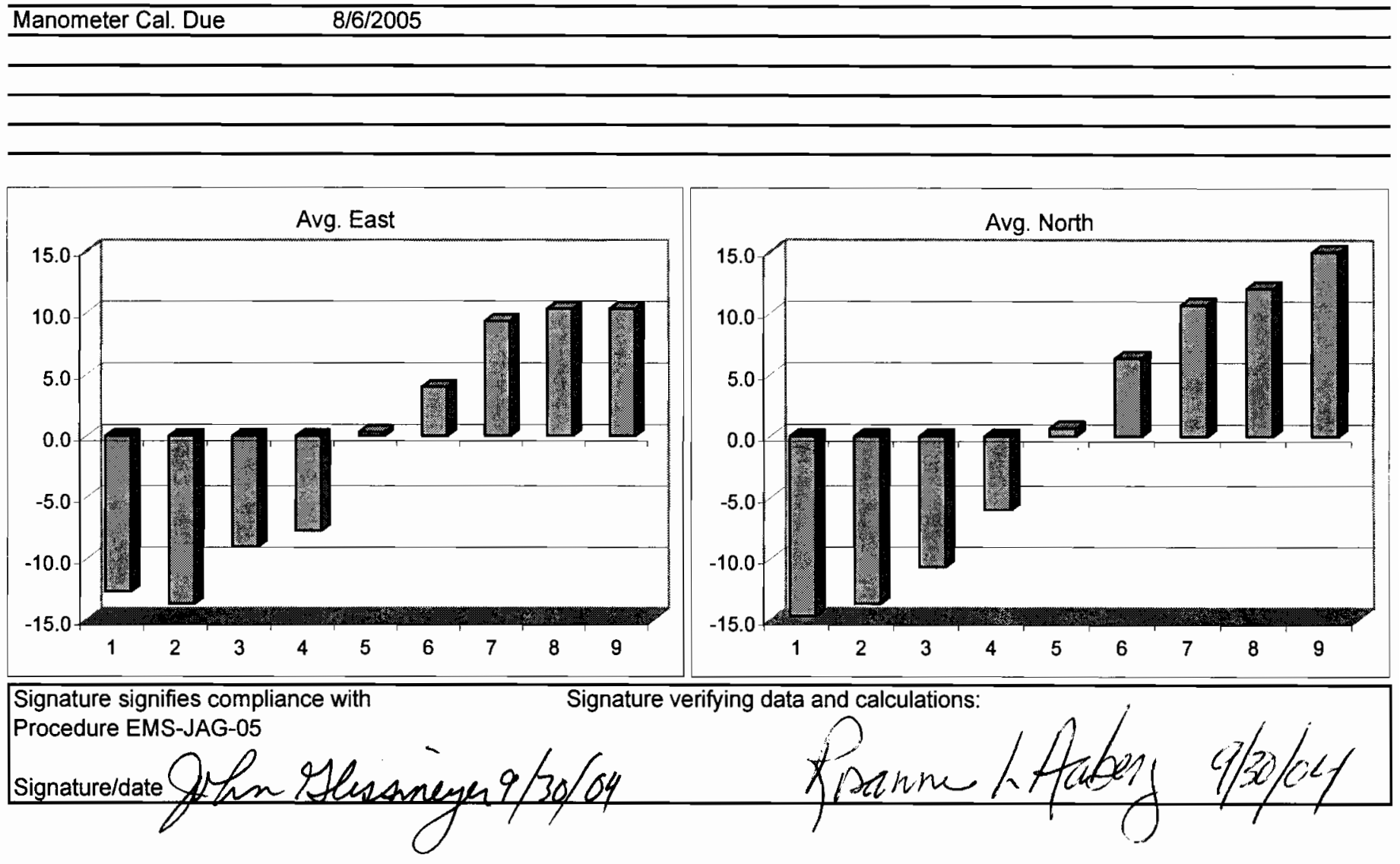


Site 325 Model

Date 8/20/2004

Tester MYB/GSH/JMB

Stack Dia.

Stack X-Area

Elevation

Distance to disturbance
Run No. FA-2

Fan Setting $37.1 \mathrm{~Hz}$

Fan configuration Fan 4 (near stack)

Approx. stack flow $1442 \quad \mathrm{cfm}$

Units degrees (clockwise > pos. nos.)

Traverse-->

Trial $\rightarrow$

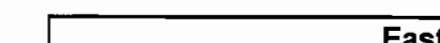

\begin{tabular}{|c|r|}
\hline Point & Depth, in. \\
\hline 1 & 0.58 \\
\hline 2 & 1.89 \\
\hline 3 & 3.49 \\
\hline 4 & 5.81 \\
\hline Center & 9.00 \\
\hline 5 & 12.19 \\
\hline 6 & 14.51 \\
\hline 7 & 16.11 \\
\hline 8 & 17.42 \\
\hline
\end{tabular}

Mean of absolute values

who points by wall:

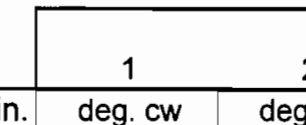
2 East 3 deg. cw

\begin{tabular}{l|r}
\hline-10 & -20 \\
-10 & -22 \\
\hline
\end{tabular}

$-22$

$-19$

-25
-18

$-19$

.00

$-5$

\begin{tabular}{|c|c|}
7 \\
\hline
\end{tabular}

.

20

21

14.6

14.0

0

5
13

17

$\frac{17}{22}$

13.1

12.3

Instuments Used:

S-type pitot

Stanley protractor level

Manometer

Pitot 2

Prot 2

Man-3 Barcode 18120

Notes:

To assure similar hose connections between the manometer and pitot tube, rotating the pitot tube assembly clockwise drives the meniscus to the right (to higher pos. numbers).

\begin{tabular}{|c|c|c|c|}
\hline \multicolumn{4}{|c|}{ North } \\
\hline deg. cw & deg. Cw & deg. Cw & Avg. \\
\hline-19 & -21 & -20 & -20.0 \\
\hline-15 & -15 & -15 & -15.0 \\
\hline-20 & -16 & -13 & -16.3 \\
\hline-9 & -15 & -8 & -10.7 \\
\hline-8 & -6 & -4 & -6.0 \\
\hline 7 & 8 & 12 & 9.0 \\
\hline 13 & 15 & 16 & 14.7 \\
\hline 19 & 15 & 18 & 17.3 \\
\hline 21 & 19 & 19 & 19.7 \\
\hline 14.6 & 14.4 & 13.9 \\
13.0 & 12.9 & 12.3 \\
\hline \multicolumn{4}{|c|}{ all } \\
\hline \multicolumn{4}{|c|}{14.3} \\
\end{tabular}

Manometer Cal. Due $8 / 6 / 2005$
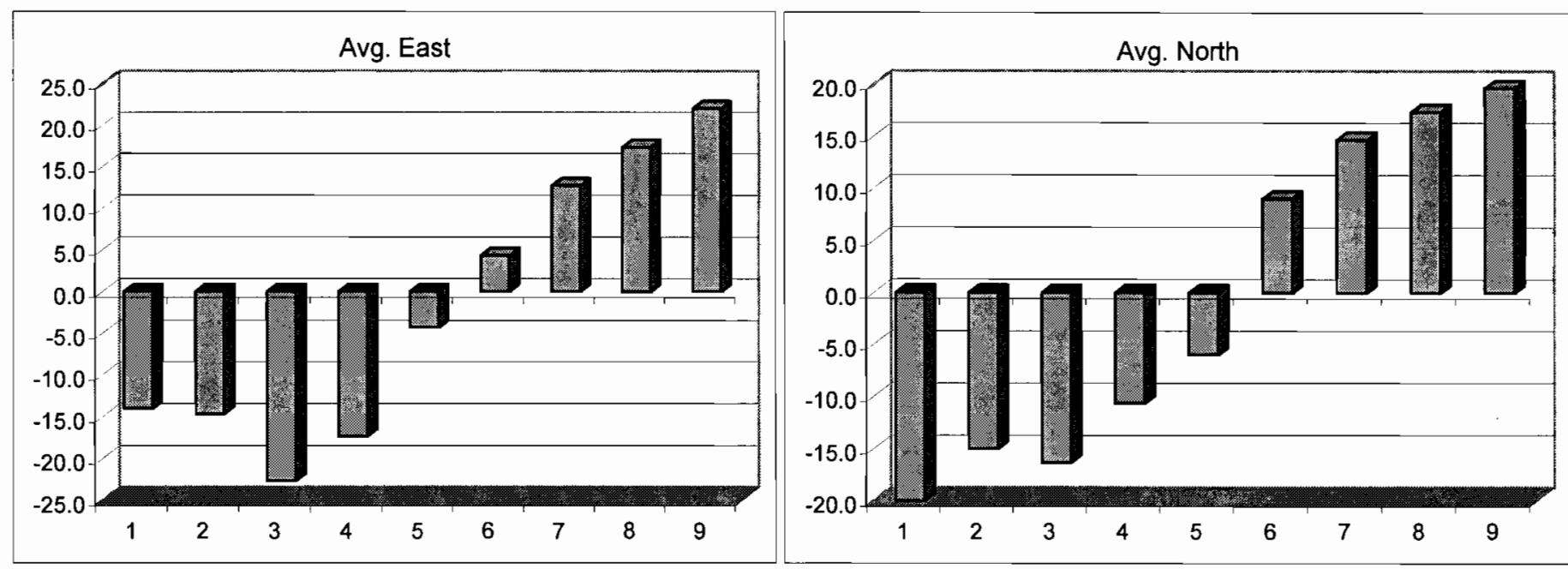

Signature signifies compliance with Procedure EMS-JAG-05

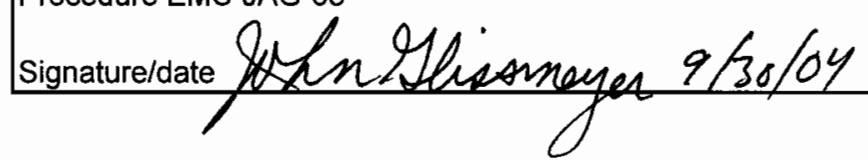

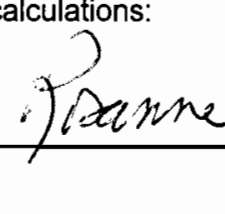

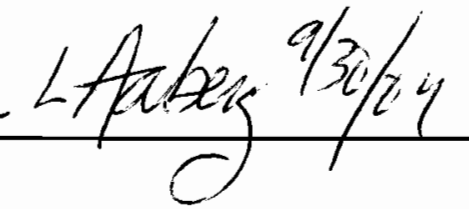


Site 325 Model

Date 8/26/2004

Tester GSH/JAG

Stack Dia.

Stack X-Area

Elevation

Distance to disturbance

\section{FLOW ANGLE DATA FORM}
Run No. FA-3
Fan Setting $37.1 \mathrm{~Hz}$
Fan configuration Near
Approx. stack flow
Units degrees (clockwise = NEG. nos.)

\begin{tabular}{|c|c|c|c|c|c|c|c|}
\hline \multirow{2}{*}{$\begin{array}{l}\text { Traverse--> } \\
\text { Trial ----> } \\
\end{array}$} & & \multicolumn{4}{|c|}{ East } & \multirow[b]{2}{*}{1} & \multirow[b]{2}{*}{2} \\
\hline & & 1 & 2 & 3 & & & \\
\hline Point & Depth, in. & deg. cw & deg. cw & deg. CW & Avg. & deg. CW & deg. \\
\hline 1 & 0.58 & -2 & -4 & -4 & -3.3 & -1 & -2 \\
\hline 2 & 1.89 & -3 & -4 & -7 & -4.7 & -3 & -4 \\
\hline 3 & 3.49 & -4 & -7 & -14 & -8.3 & -4 & -5 \\
\hline 4 & 5.81 & -3 & -6 & -11 & -6.7 & -3 & -4 \\
\hline Center & 9.00 & 0 & -3 & -3 & -2.0 & 0 & 1 \\
\hline 5 & 12.19 & 4 & 4 & 6 & 4.7 & 7 & 8 \\
\hline 6 & 14.51 & 13 & 13 & 12 & 12.7 & 13 & 15 \\
\hline 7 & 16.11 & 18 & 14 & 18 & 16.7 & 12 & 16 \\
\hline 8 & 17.42 & 22 & 20 & 18 & 20.0 & 15 & 16 \\
\hline \multicolumn{2}{|c|}{$\begin{array}{l}\text { Mean of absolute values } \\
\text { w/o points by wall: }\end{array}$} & $\begin{array}{l}7.7 \\
6.4\end{array}$ & $\begin{array}{l}8.3 \\
7.3 \\
\end{array}$ & \multicolumn{2}{|l|}{$\begin{array}{l}10.3 \\
10.1\end{array}$} & $\begin{array}{l}6.4 \\
6.0\end{array}$ & $\begin{array}{l}7.9 \\
7.6\end{array}$ \\
\hline $\begin{array}{l}\text { Instuments } \\
\text { S-type pitot } \\
\text { Stanley pro } \\
\text { Manometer }\end{array}$ & tor level & $\begin{array}{l}\text { Pitot 2 } \\
\text { Prot 2 } \\
\text { Man-3 Barc }\end{array}$ & 18120 & \multicolumn{4}{|c|}{$\begin{array}{l}\text { Notes: } \\
\text { To assure similar hose connections } \\
\text { between the manometer and pitot tube, rotating } \\
\text { the pitot tube assembly clockwise drives the } \\
\text { meniscus to the right (to higher pos. numbers). }\end{array}$} \\
\hline
\end{tabular}

Manometer Cal. Due $8 / 6 / 2005$
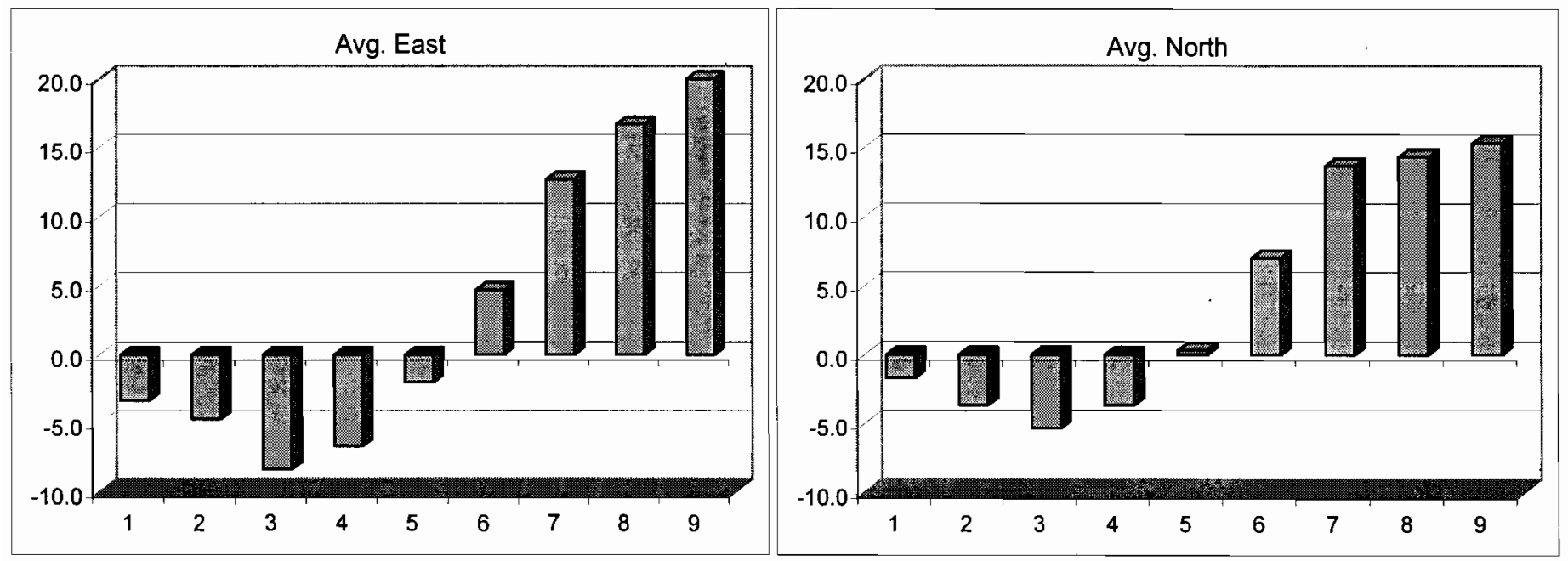

Signature signifies compliance with Procedure EMS-JAG-05

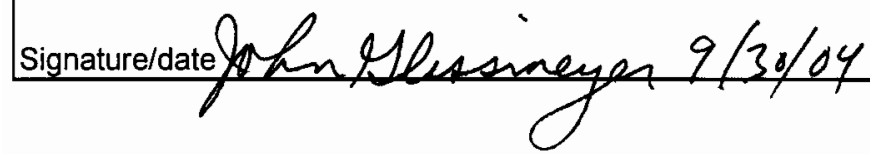

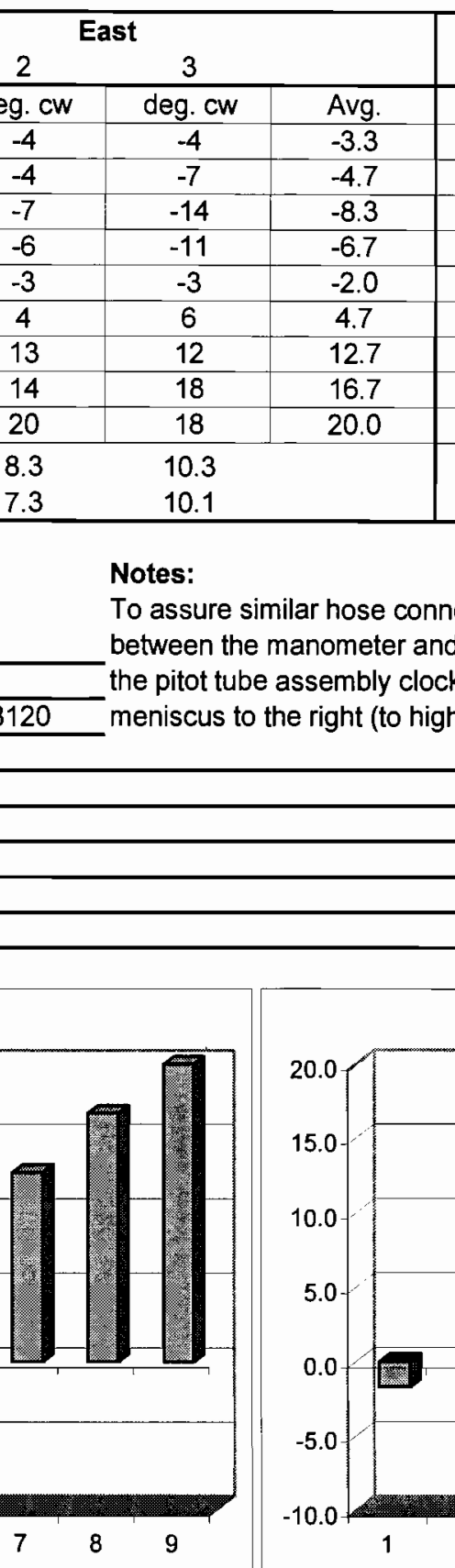

North

\begin{tabular}{|c|c|c|}
\hline 2 & 3 \\
\hline eg. $\mathrm{Cw}$ & deg. $\mathrm{Cw}$ & Avg. \\
\hline-2 & -2 & -1.7 \\
\hline-4 & -4 & -3.7 \\
\hline-5 & -7 & -5.3 \\
\hline-4 & -4 & -3.7 \\
\hline 1 & 0 & 0.3 \\
\hline 8 & 6 & 7.0 \\
\hline 15 & 13 & 13.7 \\
\hline 16 & 15 & 14.3 \\
\hline 16 & 15 & 15.3 \\
\hline 7.9 & 7.3 \\
7.6 & 7.0 \\
\hline \multicolumn{3}{|c|}{ all } \\
& \multicolumn{3}{|c|}{8.0} \\
\cline { 2 - 3 } & w/o wall pts & 7.4 \\
\hline
\end{tabular}

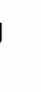




\begin{tabular}{|l|l|}
\hline \multicolumn{2}{|c|}{ Test Instruction } \\
\hline Project: 325 Stack Sampler \\
Qualification
\end{tabular}




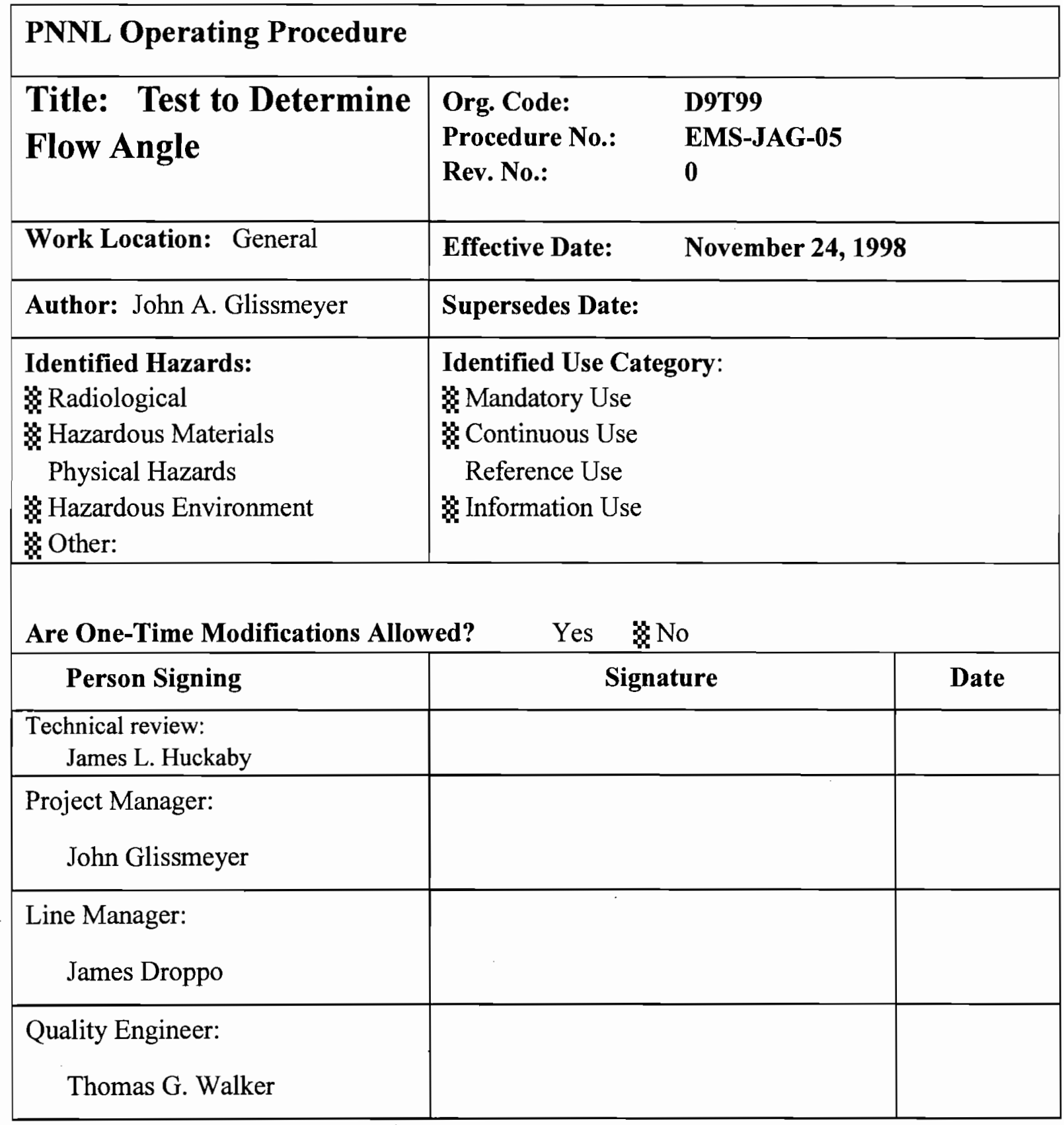




\begin{tabular}{|l|ll|}
\hline PNNL Operating Procedure & $\begin{array}{l}\text { Rev. No. } 0 \\
\text { Org. Code: D9T99 }\end{array}$ & $\begin{array}{l}\text { Page } 2 \text { of } 9 \\
\text { Procedure No.: EMS-JAG-05 }\end{array}$ \\
\hline \multicolumn{3}{|r|}{ Title: Test to Determine Flow Angle } \\
\hline
\end{tabular}

\subsection{Purpose}

The performance of new stack sampling systems must be shown to satisfy the requirements of 40 CFR 61, Subpart H, "National Emission standards for Emissions of Radionuclides Other than Radon from Department of Energy Facilities." This regulation governs portions of the design and implementation of effluent air sampling. The stack sampler performance is adequately characterized when potential contaminants in the effluent are of a uniform concentration at the sampling plane and line losses are within acceptable limits. (The sampling plane is the cross section of the stack or duct where the sampling nozzle inlet is located.) Uniformity of contaminant concentration is highly unlikely where the mean angle of the gas velocity throughout the cross section of the stack or duct is significantly non-zero. This condition would also mean that the air velocity approaches the sampling nozzle at an unacceptable angle, degrading the performance of the nozzle. This procedure provides the means to determine the mean flow angle, and is performed prior to measurements of contaminant uniformity. This procedure is performed after the range of gas flow conditions is established. Other associated procedures generally follow and address uniformity of flow and of gas and aerosol contaminants.

\subsection{Applicability}

This procedure can be used in the field or on modeled stacks and ducts to determine the angle of the air velocity relative to the axis of the duct or stack. The angle measured is the roll angle. This should be determined at the sampling plane. The tests are applicable within the following constraints:

- The operating limits of the air velocity measurement device used are observed.

- The air velocity sensor element does not occupy more than a few percent of the crosssectional area in the plane of the element.

This procedure may need to be repeated if there are changes made in the configuration of the ventilation system. If the system under test operates within a limited range of airflow that does not change more than $\pm 25 \%$, this procedure is usually conducted once at the middle of the range. If the flow varies more, the procedure is performed at least at the extremes of flow. 
Rev. No. 0

Org. Code: D9T99

Title: Test to Determine Flow Angle

\subsection{Prerequisites and Conditions}

Conditions and concerns that must be satisfied prior to performing this procedure are listed below:

- The job-hazards analysis for the work area must be prepared and followed.

- Safety glasses, hard toed or substantial shoes may be required in the work areas.

- Scaffold user training may be required to access the sampling ports of the stack.

- A ventilation flow control device must be installed and means available for its adjustment.

- Air velocity measurement equipment must be within calibration.

- The test instruction must be read and understood.

\subsection{Precautions and Limitations}

Access to the test ports may require the use of ladders, scaffolding or manlifts, which may necessitate special training for sampling personnel and any observers. The training requirements will be indicated in the job hazard analysis.

\subsection{Equipment Used for Measurements}

The following are essential items of equipment:

- A Type-S pitot tube with sufficient length to reach across the diameter of the test stack,

- Slant tube or calibrated electronic manometer to indicate when the differential pressure reading of the pitot tube is about zero,

- Device for measuring the pitot tube angle at traverse points (e.g., a protractor level with good angle resolution). (Note: A three dimensional velocity probe capable of measuring both pitch and yaw angles of gas flow is also acceptable provided that modifications in the method outlined below are made),

- Tape or template to mark insertion depths on the pitot tube,

- Velocity sensor to check the stack airflow,

- Means to obtain temperature and barometric pressure for any corrections needed for the current test conditions,

- Platform, ladders, or manlifts as needed to support equipment and to access the test ports,

- Fittings to limit leakage around the pitot tube and to stabilize the tube so that it can be positioned repeatedly in the test stack at the same location. 


\begin{tabular}{|l|ll}
\hline PNNL Operating Procedure & $\begin{array}{l}\text { Rev. No. } 0 \\
\text { Org. Code: D9T99 }\end{array}$ & Page 4 of 9 \\
& Procedure No.: EMS-JAG-05
\end{tabular}

Title: Test to Determine Flow Angle

Further details on specific equipment for the job are provided in the Test Instruction. The test method is based on 40 CFR 60, Appendix A, Method 1, Section 2.4, "Verification of the Absence of Cyclonic Flow." The measurement instrumentation may be either the type used in Method 1, or another measurement device designed for measuring the angle of the velocity vector at discrete points. The user should be aware that different devices may give different readings.

\subsection{Work Instructions for Setup, Measurements, and Data Reduction}

Job specific instructions given in the Test Instruction, illustrated in Exhibit A, will provide details and operating parameters necessary to perform this procedure. Prior to determination of flow angles, measurements should be made to assess whether the stack velocity flow is within normal limits.

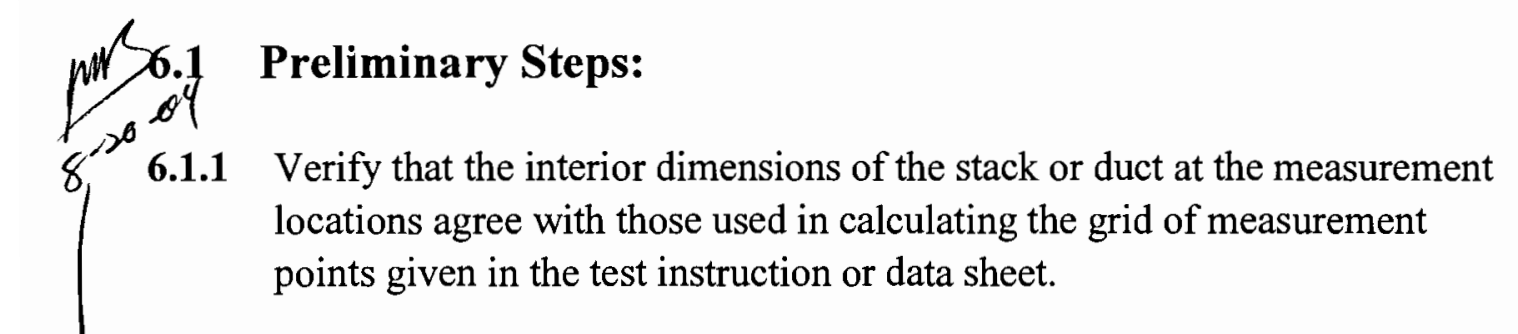

Note. The grid of measurement points is calculated in accordance with 40 CFR 60, Appendix A, Method 1. A centerpoint is included as a common reference and for graphical purposes. The layout design divides the area of the sampling plane so that each point represents approximately an equal-sized area

6.1.2 Provide essential supplies at the sampling location. (S-Type pitot tube, manometer, tubing, fittings to adapt the sensor to the test ports, marking pens, data sheets, writing and sensor supporting platforms).

6.1.3 Verify that the ventilation flow control device is capable of the flow control settings given in the Test Instruction. 
Title: Test to Determine Flow Angle

6.1.4 Prepare a data sheet for the measurement traverse. See illustration in Exhibit B. Label the columns of traverse data by the direction of the traverse. For example, if the first reading is closest to the east port, and the last reading is closest to the west port, then label the traverse "eastwest".

6.1.5 Mark the Type-S pitot tube to indicate the insertion depth for each point in the measurement grid.

6.1.6 Set the stack flow control per the test instruction. (Use a velocity or flow sensor to verify that correct flow has been achieved.)

Note. Flow verification can be based on a single point velocity reading. The single point can be the same one determined in the stack flow controller calibration in Procedure EMS-JAG-03. The barometric pressure, relative humidity, stack temperature and static pressure values may be needed to convert the velocity sensor readings to velocity units.

6.1.7 Insert the Type-S pitot tube in the stack or duct, seal the opening around it, and check for smooth operation of the pitot tube.

Note. Good measurements are dependent upon making small repeatable rotations of the pitot tube in the available fittings.

6.1.8 Establish a convention for representing the angular direction of flow.

Note. If an inclined manometer is used, connect the flexible tubes between the connectors on the pitot tube and the manometer so that rotating the pitot tube assembly clockwise drives the meniscus to the right, i.e., to higher positive numbers.

Attach a circular protractor to the pitot tube near the tubing connectors. Generally the protractor hangs below the pitot tubes. When the parallel tubes are in horizontal position, the protractor should indicate zero degrees. If the tubing assembly is rotated clockwise, the resulting counter-clockwise movement of the angle indicator produces an angle that is read as a positive number. This is consistent with the convention for reading circular angles. 
Title: Test to Determine Flow Angle

6.1.9 Position the inclined manometer on a stable platform and level the device using the spirit level.

Note: Movement on the test platform may affect the manometer level. It should be checked frequently. Adjustments can be made at any time when the pitot tube is moved to the next position, but not during readings at any single point.

6.1.10 Connect the flexible tubes to the inclined manometer but disconnect them from the pitot tube.

6.1.11 Increase or decrease the red oil level in the inclined portion of the manometer to zero the meniscus. (This is done using a finger-adjustable screw at the base of the manometer.)

6.1.12 Reconnect the flexible tubes to the pitot tube.

\subsection{Angular Flow Measurements}

6.2.1 Verify that the directional orientations and the numbered measurement positions are consistent with the data sheet.

6.2.2 Measure and record, on the data sheet, the angular reading at each measurement point in succession. If the readout device has an averaging feature, record the average of a series of several readings.

Note: Each test relies on one repetition for each measurement point in each traverse direction, repeated three times. The repeats are made as three separate runs and not as three consecutive measurements at each point.

The readings may be erratic for some flow conditions and at some traverse positions. Care should be taken to approach these variable readings from both higher and lower angles to obtain the most accurate equilibrium reading. 
Title: Test to Determine Flow Angle

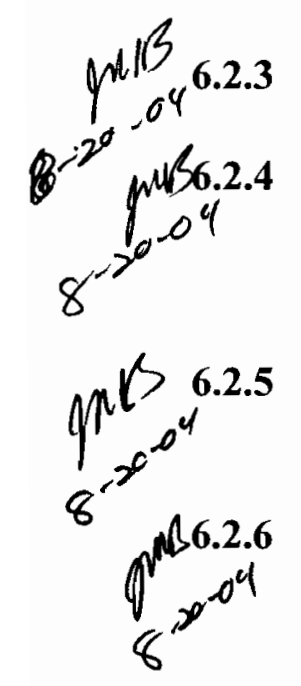

Repeat Step 6.3.3.

Compare the results in Step 6.3.4 with those of 6.3.3. If the measurements are not highly reproducible, repeat Step 6.3.3 again.

Note: The acceptance criterion is that the average flow angle not exceed 20 degrees.

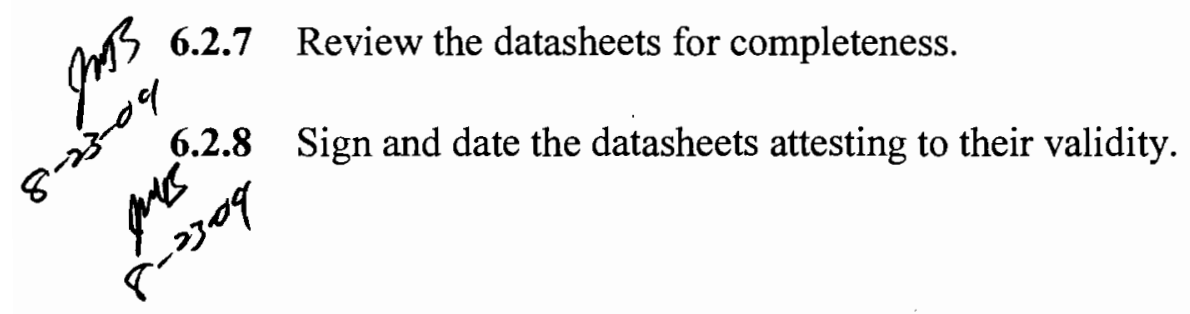




\begin{tabular}{|l|ll|}
\hline PNNL Operating Procedure & $\begin{array}{l}\text { Rev. No. } 0 \\
\text { Org. Code: D9T99 }\end{array}$ & $\begin{array}{l}\text { Page 8 of 9 } \\
\text { Procedure No.: EMS-JAG-05 }\end{array}$ \\
\hline
\end{tabular}

\subsection{Exhibits/Attachments}

Exhibit A - Illustrative Test Instruction

\begin{tabular}{|c|c|c|}
\hline \multicolumn{3}{|c|}{ Test Instruction } \\
\hline $\begin{array}{l}\text { Project: W420 6" Stack } \\
\text { Calibration } 28361\end{array}$ & Date: August 19, 1998 & Work Package: K83017 \\
\hline \multicolumn{3}{|c|}{ Tests: Flow Angle at High Flow in W420 6" Full-Scale Model Stack } \\
\hline \multicolumn{3}{|l|}{ Staff: David Maughan } \\
\hline \multicolumn{3}{|c|}{$\begin{array}{l}\text { Reference Procedures: } \\
\text { 1. Operating Manual for Solomat Zephyr } \\
\text { 2. Test to Determine Flow Angle at the Elevation of a Sampler Probe, Procedure EMS-JAG- } \\
05\end{array}$} \\
\hline \multicolumn{3}{|c|}{$\begin{array}{l}\text { Equipment: } \\
\text { 1. W420 6" Full-Scale Model Stack, Fan and Fan Speed Controller located in } 305 \text { Bldg. } \\
\text { 2. S-type Pitot Tube, slant tube or electronic manometer, and Protractor Level }\end{array}$} \\
\hline \multicolumn{3}{|c|}{$\begin{array}{l}\text { Safety Considerations: } \\
\text { Review and observe the applicable Numatec Job Hazard Analysis for the project }\end{array}$} \\
\hline \multicolumn{3}{|c|}{$\begin{array}{l}\text { Instructions: } \\
\text { 5. Assemble the equipment for the flow angle test at the ports at the elevation of the sampling } \\
\text { probe. } \\
\text { 2. Layout the measurement points with the following distances from the inside of the stack } \\
\text { wall: } 0.5,0.66,1.23,2.04,3.16,4.28,5.10,5.66,5.83 \text { inches. } 3 \text {. Measure the flow angle at } \\
\text { each point at the high }(400 \mathrm{cfm}) \text { extreme of stack flow. Repeat each measurement twice. } \\
\text { 4. Record the data on flow angle data sheets. } \\
\text { 5. Diagram mounting fixtures and retain assembly for subsequent tests }\end{array}$} \\
\hline \multicolumn{3}{|c|}{ Desired Completion Date: $12 / 5 / 98$} \\
\hline Approvals: & & \\
\hline John Glissm & project manager & Date \\
\hline Test completed by: & & Date: \\
\hline
\end{tabular}


Rev. No. 0

Org. Code: D9T99
Page 9 of 9

Procedure No.: EMS-JAG-05

\section{Title: Test to Determine Flow Angle}

\section{Exhibit B - Illus tration of Flow Angle Data Sheet}

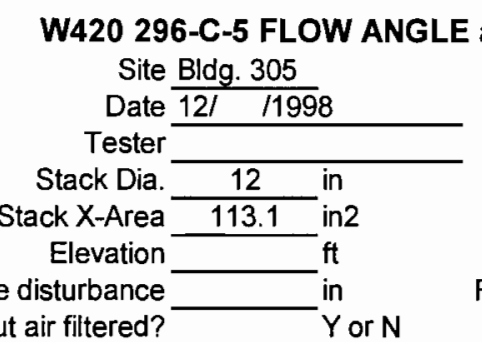

$\begin{aligned} & \text { at High and Low Average Flow Rates } \\ & \text { Run No. } \\ & \text { Stack Temp } \\ & \text { Stack RH } \\ & \text { Baro Press } \\ & \text { Fan Setting } \text { percent } \\ & \text { Fan input port } \text { mbar } \\ & \text { Flowrate (pre- \& post-) } \text { and } \\ & \text { Approx. avg. Flowrate } \text { cfm at centerline }\end{aligned}$

El. above disturbance Approx. avg. Flowrate

Units degrees (clockwise > pos. nos.)

\begin{tabular}{|c|c|c|c|c|c|c|c|}
\hline \multirow{3}{*}{\multicolumn{2}{|c|}{$\begin{array}{l}\text { Traverse--> } \\
\text { Trial --> } \\
\quad \text { Point Depth, in. }\end{array}$}} & \multicolumn{3}{|c|}{ East } & \multicolumn{3}{|c|}{ South } \\
\hline & & \multicolumn{2}{|c|}{1} & 3 & 1 & 2 & 3 \\
\hline & & deg. Cw & deg. cw & deg. cw & deg. cw & deg. cw & deg. Cw \\
\hline 1 & 0.50 & & & & & & \\
\hline 2 & 0.80 & & & & & & \\
\hline 3 & 1.42 & & & & & & \\
\hline 4 & 2.12 & & & & & & \\
\hline 5 & 3.00 & & & & & & \\
\hline 6 & 4.27 & & & & & & \\
\hline CenterPt. & 6.00 & & & & & & \\
\hline 7 & 7.77 & & & & & & \\
\hline 8 & 9.00 & & & & & & \\
\hline 9 & 9.88 & & & & & & \\
\hline 10 & 10.58 & & & & & & \\
\hline 11 & 11.20 & & & & & & \\
\hline 12 & 11.50 & & & & & & \\
\hline & & & West & & & North & \\
\hline all data: & or & 0.0 & 0.0 & 0.0 & 0.0 & 0.0 & 0.0 \\
\hline
\end{tabular}

Instuments Used:

Parallel-tube pitot with 90-deg. bends at sample ends, 24-inches in length.

Dwyer Instruments 0-5 inch inclined manometer with red guage oil

zero'd and leveled (with connecting tubes open to room atmosphere).

Angles made using Empire \#36 circular protractor.

Notes:

To assure similar hose connections between the manometer and pitot tube, rotating the pitot tube assemble clockwise drives the meniscus to the right (to higher pos. numbers).
Cal Exp. Date: NA

Signature signifies compliance with Procedure EMS-JAG-05
Signature/date


Appendix E

Tracer Gas Uniformity Procedure and Data 



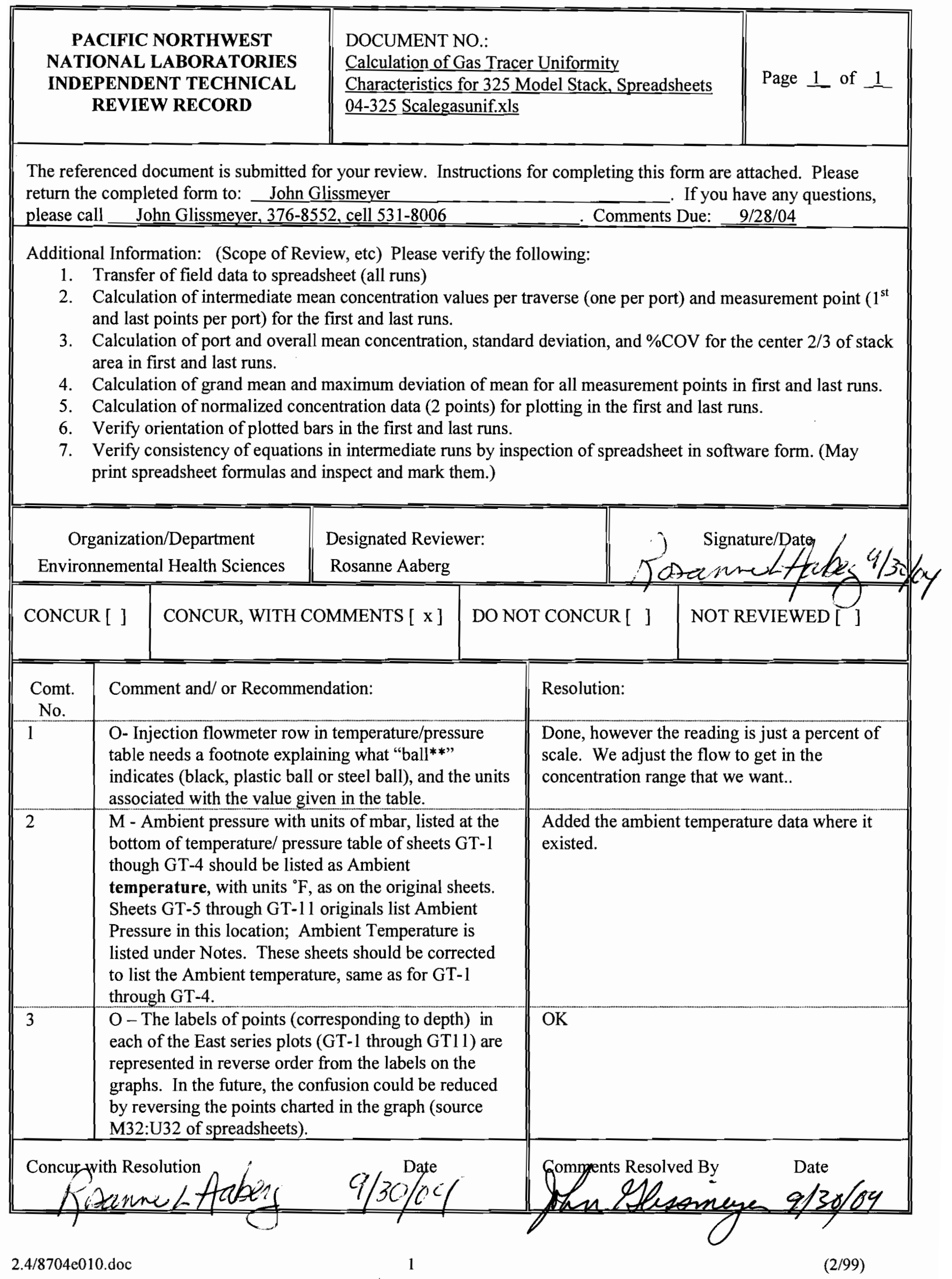


TRACER GAS TRAVERSE DATA FORM

\begin{tabular}{|c|c|}
\hline \multicolumn{2}{|c|}{ Site 325 Model } \\
\hline Date & $9 / 1 / 2004$ \\
\hline \multicolumn{2}{|c|}{ Tester JAG/GSH/JMB } \\
\hline Stack Dia. & 18 in. \\
\hline Stack X-Area & 254.5 in. 2 \\
\hline \multicolumn{2}{|c|}{ Elevation N.A. } \\
\hline Distance to disturbance & 120 inches \\
\hline
\end{tabular}

Run No. GT-1

Fan Configuration Near fan

Fan Setting $37.1 \mathrm{~Hz}$

Stack Temp

$85 \operatorname{deg} F$

Start/End Time $1500 / 1630$

Center $2 / 3$ from 1.65 to: 16.35

Points in Center $2 / \overline{2}$ to: $\overline{7}$

Injection Point Top South

\begin{tabular}{|c|c|c|c|c|c|c|c|c|c|}
\hline \multirow{2}{*}{$\begin{array}{l}\text { Traverse--> } \\
\text { Trial } \longrightarrow\end{array}$} & & \multicolumn{4}{|c|}{ East } & \multicolumn{4}{|c|}{ North } \\
\hline & & 1 & 2 & 3 & Mean & 1 & 2 & 3 & Mean \\
\hline $\begin{array}{r}\text { Point } \\
\end{array}$ & Depth, in. & \multicolumn{4}{|c|}{$\mathrm{ppm}$} & \multicolumn{4}{|c|}{$\mathrm{ppm}$} \\
\hline 1 & 0.58 & 3.55 & 3.03 & 3.24 & 3.27 & 3.25 & 3.27 & 2.94 & 3.15 \\
\hline 2 & 1.89 & $3 . \overline{91}$ & 3.26 & 3.09 & 3.42 & 3.10 & 2.80 & 3.05 & 2.98 \\
\hline 3 & 3.49 & $3 . \overline{12}$ & 2.89 & $3 . \overline{12}$ & 3.04 & 3.00 & $2.8 \overline{6}$ & 3.24 & 3.03 \\
\hline 4 & 5.81 & 3.28 & 3.01 & 2.97 & 3.09 & 3.02 & 3.01 & 2.84 & 2.96 \\
\hline Center & 9.00 & 2.84 & 2.93 & 3.14 & 2.97 & 3.00 & 3.01 & 2.85 & 2.95 \\
\hline-5 & 12.19 & 2.94 & 2.88 & 2.90 & 2.91 & 2.79 & 2.90 & 2.96 & $2 . \overline{88}$ \\
\hline$\overline{6}$ & 14.51 & 2.76 & 2.87 & 2.86 & 2.83 & $2 . \overline{94}$ & 3.24 & 2.95 & 3.04 \\
\hline 7 & 16.11 & 3.08 & 3.38 & 2.80 & 3.09 & 2.81 & 2.86 & 2.99 & 2.89 \\
\hline 8 & 17.42 & 3.33 & $2 . \overline{98}$ & 3.02 & 3.11 & 2.97 & 3.09 & 3.10 & 3.05 \\
\hline Averages - & $\longrightarrow$ & 3.20 & 3.03 & 3.02 & 3.08 & 2.99 & 3.00 & 2.99 & 2.99 \\
\hline
\end{tabular}

\begin{tabular}{|llrl|llr|}
\hline All & $\frac{\mathrm{ppm}}{3.04}$ & Dev. from mean & Center 2/3 & East & $\frac{\text { North }}{2.05}$ & All \\
Mean & 3.04 & & Mean & 3.05 & 2.96 & 3.01 \\
Min Point & 2.83 & $-6.8 \%$ & Std. Dev. & 0.19 & 0.06 & 0.14 \\
Max Point & 3.42 & $12.6 \%$ & CoV as $\%$ & 6.21 & 2.14 & $\mathbf{4 . 7 5}$ \\
\hline
\end{tabular}

Avg. Conc. $\quad 3.047 \mathrm{ppm}$

Tracer tank pressure

Sample Port Temp

Centerline vel.

Injection flowmeter

Stack flow

Sampling flowmeter

Ambient pressure

Ambient humidity

$B \& K$ vapor correction

Back-Gd gas level

No. Bk-Gd samples

Ambient temp.

Instuments Used:

B \& K Model $1302 \# 1765299$

Sierra Inc. Constant Flow Air Sampler

Solomat Zephyr SN 1295-1472

${ }^{\star \star}$ Reading on black plastic ball float in flowmeter

Notes: At this point, the duct is $18 \times 18$ inches

The injection probe reaches 8 -in off center, so with the

elevation adjusted accordingly, the injection is one-inch

from both sides of a corner. Also, the center injection

point is 8-in closer to the stack than the injection port.
Gas analyzer checked:

9/1/2004

Wind WSW $18 \mathrm{mph}$, gusts to $25 \mathrm{mph}$

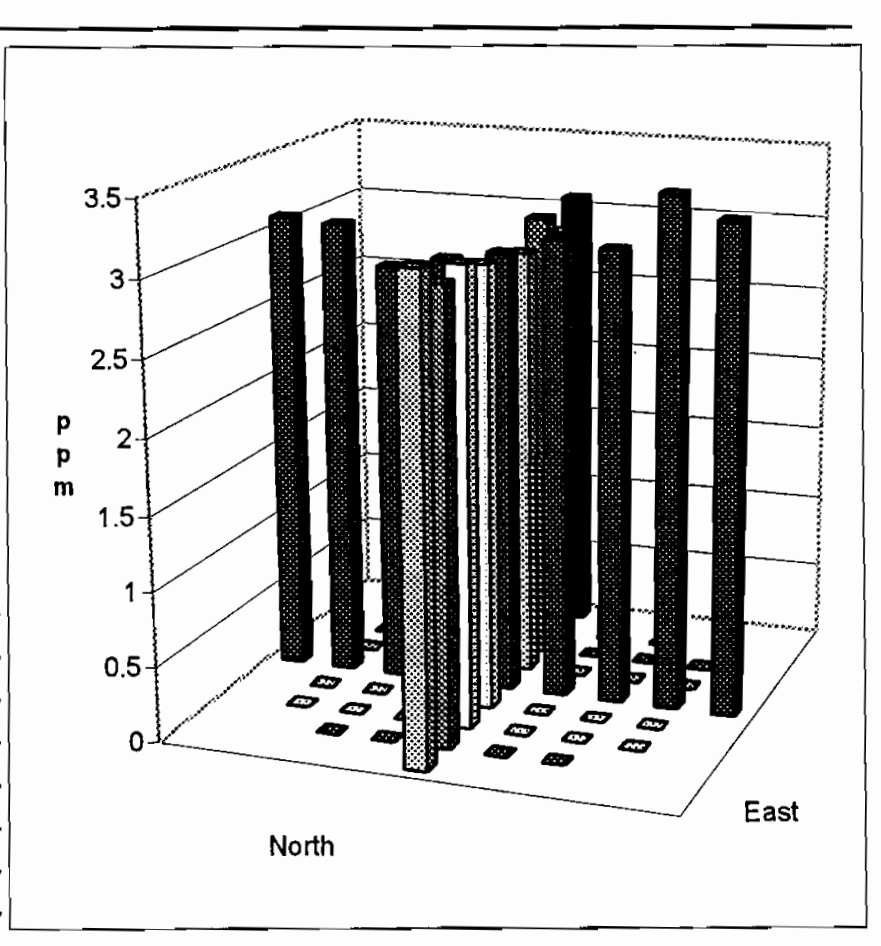

Signature signifies compliance with Signature verifying data and calculations:

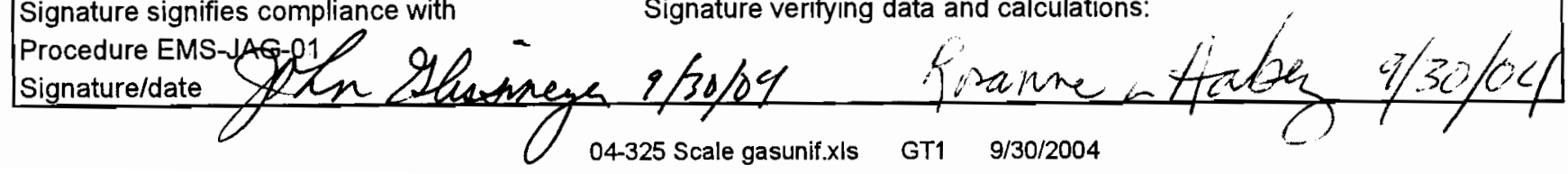


TRACER GAS TRAVERSE DATA FORM

Site 325 Model

Date 9/2/2004

Tester JAG/GSH/JMB

Stack Dia.

Stack X-Area 18 in.

Elevation N.A.

Distance to disturbance

Measurement units ppm SF6
Run No. GT-2

Fan Configuration Near fan

Fan Setting $37.1 \mathrm{~Hz}$

Stack Temp

Start/End Time $\overline{1347 / 1445}$

Center $2 / 3$ from

Points in Center $2 / 3$

Injection Point Top North - 1 -in from walls at corner

\begin{tabular}{|c|c|c|c|c|c|c|c|c|c|}
\hline & & & & & & \multirow{2}{*}{\multicolumn{4}{|c|}{$\overline{\text { North }}$}} \\
\hline \multirow{2}{*}{\multicolumn{2}{|c|}{$\begin{array}{l}\text { Traverse--> } \\
\text { Trial } \longrightarrow \\
\end{array}$}} & \multicolumn{4}{|c|}{ East } & & & & \\
\hline & & \multirow[t]{2}{*}{1} & \multirow{2}{*}{\multicolumn{2}{|c|}{2}} & \multirow[t]{2}{*}{ Mean } & 1 & & 3 & Mean \\
\hline Point & Depth, in. & & & & & \multicolumn{4}{|c|}{$\frac{2}{\mathrm{ppm}}$} \\
\hline 1 & 0.58 & 3.42 & 3.70 & 3.71 & 3.61 & 3.68 & 3.64 & 3.60 & 3.64 \\
\hline 2 & 1.89 & 3.55 & 3.86 & 3.56 & 3.66 & 3.68 & 3.57 & 3.43 & 3.56 \\
\hline 3 & 3.49 & 3.65 & 3.71 & 3.44 & 3.60 & 3.64 & 3.37 & $3 . \overline{43}$ & 3.48 \\
\hline 4 & 5.81 & 3.50 & 3.70 & 3.46 & 3.55 & 3.62 & 3.43 & 3.43 & $\overline{3.49}$ \\
\hline Center & 9.00 & 3.56 & 3.63 & $\overline{3.66}$ & 3.62 & 3.64 & 3.33 & 3.19 & 3.39 \\
\hline 5 & 12.19 & 3.65 & $3 . \overline{79}$ & 3.48 & 3.64 & 3.63 & 3.55 & 3.34 & 3.51 \\
\hline 6 & 14.51 & 3.61 & 3.49 & 3.38 & 3.49 & 3.54 & 3.32 & 3.33 & 3.40 \\
\hline 7 & $16 . \overline{11}$ & 3.66 & 3.63 & 3.42 & 3.57 & 3.54 & 3.35 & 3.36 & $3.4 \overline{2}$ \\
\hline 8 & 17.42 & 3.63 & 3.57 & 3.30 & 3.50 & 3.64 & 3.51 & 3.30 & 3.48 \\
\hline \multicolumn{2}{|c|}{ Averages $\longrightarrow$} & \begin{tabular}{l|l|}
3.58 \\
\end{tabular} & 3.68 & 3.49 & 3.58 & 3.62 & 3.45 & 3.38 & 3.48 \\
\hline
\end{tabular}

Avg. Conc.

\begin{tabular}{|lr}
\hline All & ppm \\
Mean & 3.53 \\
Min Point & 3.39 \\
Max Point & 3.66 \\
\hline
\end{tabular}

$3.538 \mathrm{ppm}$

Tracer tank pressure Sample Port Temp Centerline vel. Injection flowmeter Stack flow

Sampling flowmeter

Ambient pressure

Ambient humidity

B\&K vapor correction

Back-Gd gas level No. Bk-Gd samples Ambient temp.

Instuments Used:

B \& K Model $1302 \# 1765299$

Sierra Inc. Constant Flow Air Sampler

Solomat Zephyr SN 1295-1472

${ }^{\star *}$ Reading on black plastic ball float in flowmeter

Notes: At this point, the duct is $18 \times 18$ inches

The injection probe reaches 8-in off center, so with the elevation adjusted accordingly, the injection is one-inch

from both sides of a corner. Also, the center injection point is 8-in closer to the stack than the injection port.

\begin{tabular}{r|llll|}
\hline Dev. from mean & Center 2/3 & East & North & All \\
\cline { 2 - 3 } & Mean & 3.59 & 3.46 & 3.53 \\
$3.5 \%$ & Std. Dev. & 0.06 & 0.06 & 0.09 \\
\hline COV as \% & 1.56 & 1.86 & 2.49 \\
\hline
\end{tabular}

Gas analyzer checked:

$9 / 1 / 2004$

Wind WSW $18 \mathrm{mph}$, gusts to $25 \mathrm{mph}$

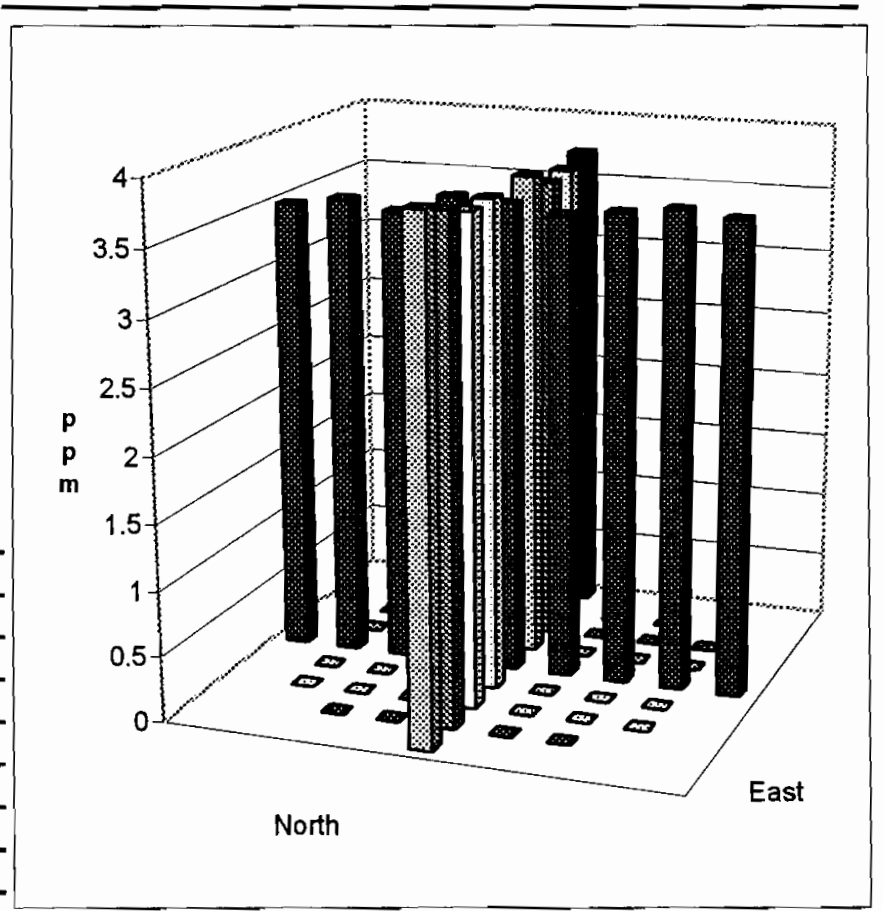

Signature signifies compliance with Signature verifying data and calculations:

Procedure EMS-DAG-01 Shature/date 
TRACER GAS TRAVERSE DATA FORM

Site 325 Model
Date $\frac{9 / 2 / 2004}{\text { Tester JAG/GSH/JMB }}$
Stack Dia. $\frac{18 \text { in. }}{254.5 \text { in.2 }}$
Stack X-Area
Elevation N.A.
Distance to disturbance
Measurement units ppm SF6

Run No. GT-3

Fan Configuration Near fan

Fan Setting $37.1 \mathrm{~Hz}$

Stack Temp

$87 \operatorname{deg} F$

Start/End Time 1445/1525

Center $2 / 3$ from 1.65 to: 16.35

Points in Center $2 / 3 \longdiv { 2 }$ to: $\underline{7}$

Injection Point Center

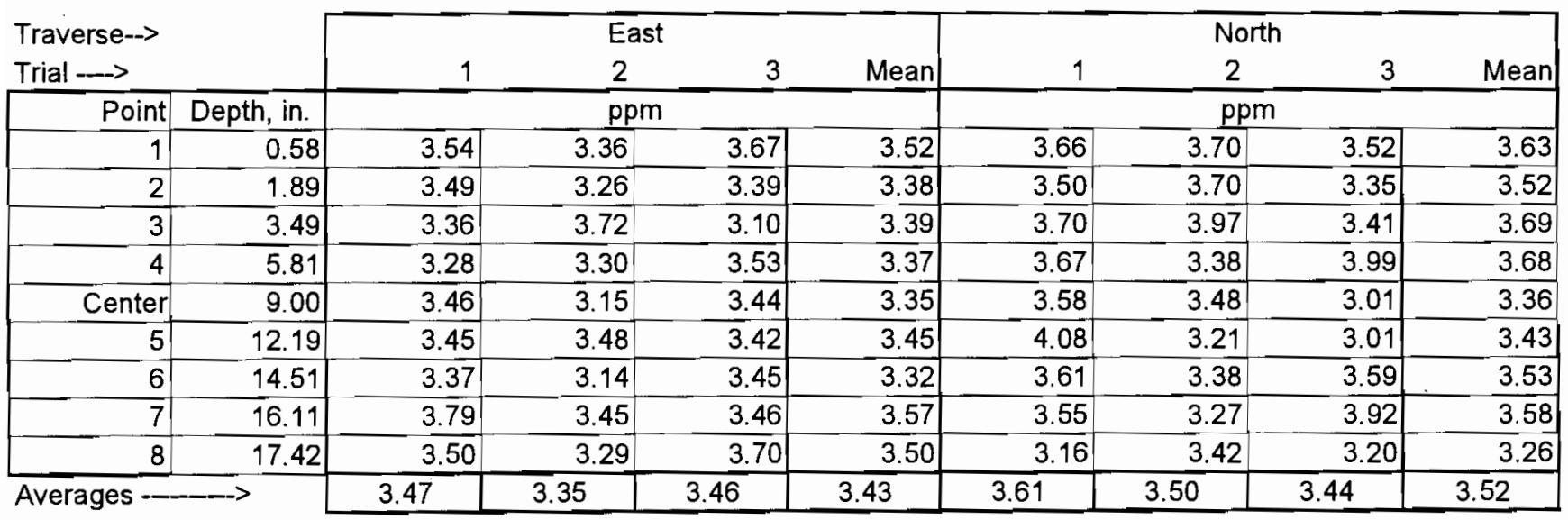

Avg. Conc. $\quad 3.489 \mathrm{ppm}$

\begin{tabular}{|lrr|llll|}
\hline All & $\mathrm{ppm}$ & Dev from mean & Center 2/3 & East & North & All \\
Mean & 3.47 & & Mean & 3.40 & 3.54 & 3.47 \\
Min Point & 3.26 & $-6.1 \%$ & Std. Dev. & 0.08 & 0.12 & 0.12 \\
Max Point & 3.69 & $6.3 \%$ & CoV as $\%$ & 2.41 & 3.47 & 3.54 \\
\hline
\end{tabular}

Gas analyzer checked:

$9 / 1 / 2004$

Tracer tank pressure Sample Port Temp

Centerline vel.

Injection flowmeter

Stack flow

Sampling flowmeter

Ambient pressure

Ambient humidity

B\&K vapor correction

Back-Gd gas level

No. Bk-Gd samples

Ambient temp.

\begin{tabular}{|c|c|c|}
\hline Start & Finish & \\
\hline 315 & 315 & $5 \mathrm{psig}$ \\
\hline 86 & & $\mathrm{~F}$ \\
\hline 830 & 779.0 & $\mathrm{fpm}$ \\
\hline 3 & & $\%$ bl. ball* \\
\hline & & $\mathrm{cfm}$ \\
\hline 10 & 10 & Ipm Sierra \\
\hline 29.54 & 29.5 & $\mathrm{~mm} \mathrm{Hg}$ \\
\hline 27 & 24 & $\% \mathrm{RH}$ \\
\hline$=\quad N$ & $\bar{N}$ & J Y/N \\
\hline $29 / 21 / 23 / 211$ & $25 / 20 / 24 / 22 l$ & \\
\hline 21 & 23 & $p p b$ \\
\hline 5 & 5 & $n$ \\
\hline 75.0 & 76.0 & deg. $F$ \\
\hline
\end{tabular}

Instuments Used:

B \& K Model 1302 \#1765299

Sierra Inc. Constant Flow Air Sampler

Solomat Zephyr SN 1295-1472

** Reading on black plastic ball float in flowmeter

Notes: At this point, the duct is $18 \times 18$ inches

The injection probe reaches 8 -in off center, so with the

elevation adjusted accordingly, the injection is one-inch

from both sides of a corner. Also, the center injection

point is 8-in closer to the stack than the injection port.
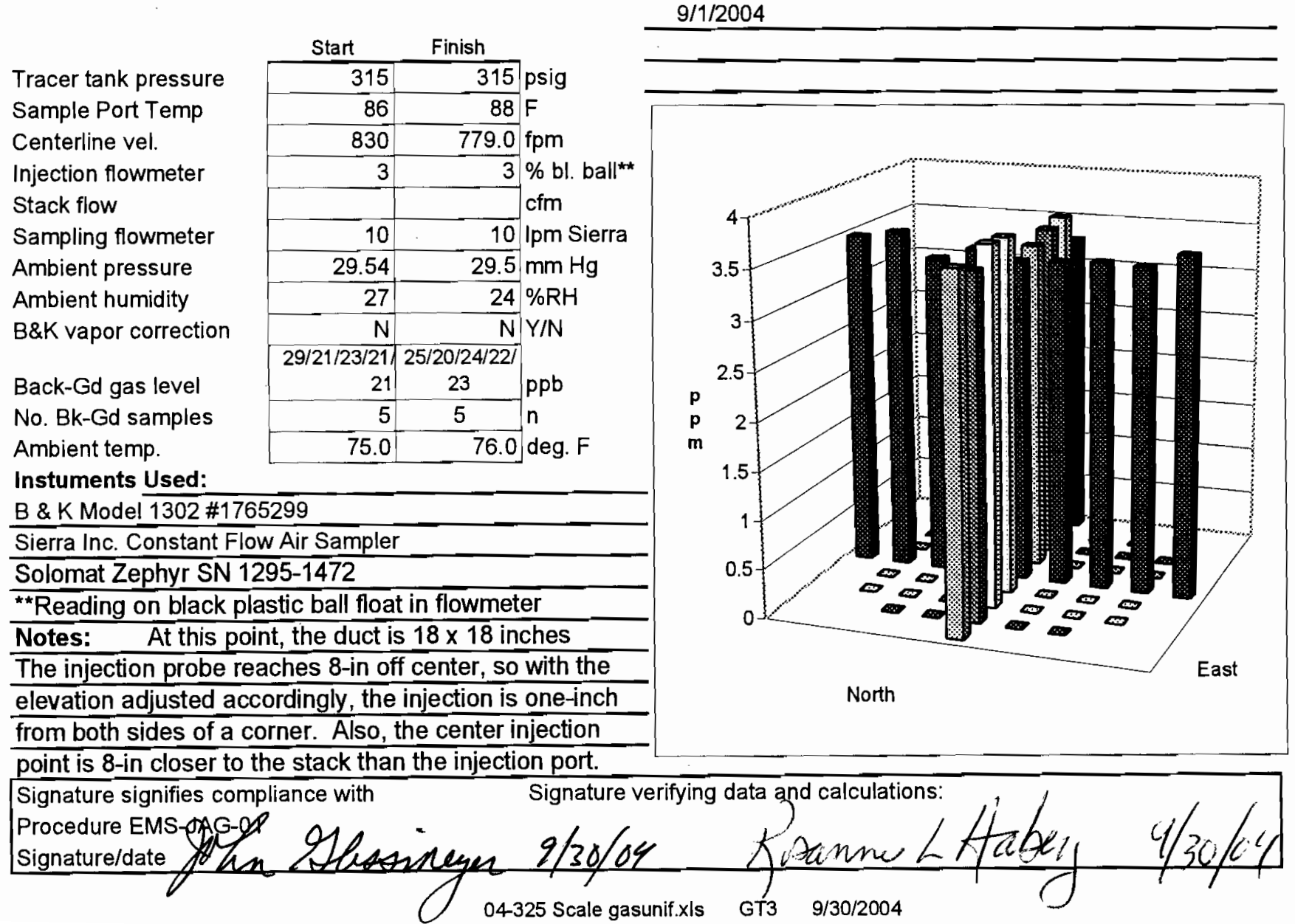
TRACER GAS TRAVERSE DATA FORM

Site 325 Model

Date 9/2/2004

Tester JAG/GSH/JMB

Stack Dia. 18 in.

Stack X-Area 254.5 in. 2

Elevation N.A.

Distance to disturbance 120 inches

Measurement units ppm SF6
Run No. GT-4

Fan Configuration Near fan

Fan Setting $37.1 \mathrm{~Hz}$

Stack Temp $84.5 \operatorname{deg} F$

Start/End Time $1525 / 1606$

Center $2 / 3$ from

Points in Center 2/3 $\begin{array}{lll}\frac{1.65}{2} & \text { to: } & \frac{16.35}{7} \\ \text { South } & \text { to: } 1 \text {-in from corner wails }\end{array}$

Injection Point Bottom South - 1-in from corner walls

\begin{tabular}{|c|c|c|c|c|c|c|c|c|c|}
\hline \multirow{2}{*}{\multicolumn{2}{|c|}{$\begin{array}{l}\text { Traverse--> } \\
\text { Trial } \longrightarrow\end{array}$}} & \multicolumn{4}{|c|}{ East } & \multicolumn{4}{|c|}{ North } \\
\hline & & 1 & 2 & 3 & Mean & 1 & 2 & 3 & Mean \\
\hline Point & Depth, in. & \multicolumn{4}{|c|}{$\mathrm{ppm}$} & \multicolumn{4}{|c|}{$\mathrm{ppm}$} \\
\hline 1 & 0.58 & 4.23 & 3.64 & 4.27 & 4.05 & 3.73 & 3.69 & 3.72 & 3.71 \\
\hline 2 & 1.89 & 4.00 & 4.41 & 3.61 & 4.01 & 3.54 & 3.73 & 3.70 & 3.66 \\
\hline 3 & 3.49 & 3.76 & 4.14 & 4.14 & 4.01 & 3.49 & 3.94 & 3.89 & 3.77 \\
\hline 4 & 5.81 & 3.92 & 3.51 & 3.73 & $3 . \overline{72}$ & 3.73 & 3.64 & 3.53 & 3.63 \\
\hline Center & 9.00 & 3.97 & 4.15 & 3.60 & 3.91 & 3.89 & 4.05 & 4.23 & 4.06 \\
\hline 5 & 12.19 & 3.63 & 4.10 & 4.41 & 4.05 & 3.81 & 3.95 & 3.96 & 3.91 \\
\hline 6 & 14.51 & 4.22 & 3.93 & 3.98 & 4.04 & 3.77 & 4.17 & 3.78 & 3.91 \\
\hline 7 & 16.11 & 3.91 & 3.97 & 4.01 & 3.96 & 3.56 & 3.96 & 4.00 & 3.84 \\
\hline 8 & 17.42 & 4.09 & 3.58 & 4.22 & 3.96 & 3.67 & 4.08 & 4.48 & 4.08 \\
\hline Averages & $7-1$ & 3.97 & 3.94 & 4.00 & 3.97 & 3.69 & 3.91 & 3.92 & 3.84 \\
\hline
\end{tabular}

Avg. Conc. $\quad 3.894 \mathrm{ppm}$

\begin{tabular}{|ll}
\hline All & ppm \\
Mean & 3.90 \\
Min Point & 3.63 \\
Max Point & 4.08 \\
\hline
\end{tabular}

Tracer tank pressure

Sample Port Temp

Centerline vel.

Injection flowmeter

Stack flow

Sampling flowmeter

Ambient pressure

Ambient humidity

B\&K vapor correction

Back-Gd gas level

No. Bk-Gd samples

Ambient temp.

Instuments Used:

B \& K Model 1302\#1765299

Sierra Inc. Constant Flow Air Sampler

Solomat Zephyr SN 1295-1472

** Reading on black plastic ball float in flowmeter

Notes: At this point, the duct is $18 \times 18$ inches

The injection probe reaches 8 -in off center, so with the

elevation adjusted accordingly, the injection is one-inch

from both sides of a corner. Also, the center injection

point is 8-in closer to the stack than the injection port.

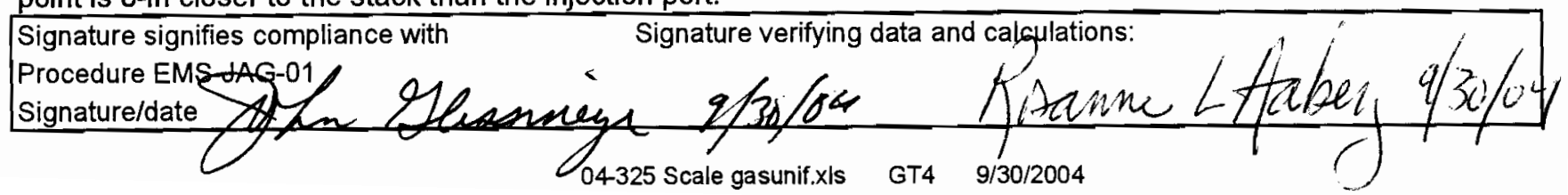


TRACER GAS TRAVERSE DATA FORM

Site 325 Model

Date 9/3/2004

Tester JAG/JMB

Stack Dia.

Stack X-Area 254.5 in. 2

Elevation N.A.

Distance to disturbance 120 inches

Measurement units ppm SF6
Run No. GT-5

Fan Configuration Near fan

Fan Setting $37.1 \mathrm{~Hz}$

Stack Temp $84.8 \operatorname{deg} \mathrm{F}$

Start/End Time $1404 / 1504$

Center $2 / 3$ from $\quad 1.65$ to: 16.35

Points in Center $2 / 3 \longdiv { 2 }$ to: $\overline{7}$

Injection Point Bottom North - 1-in from corner walls

\begin{tabular}{|c|c|c|c|c|c|c|c|c|c|}
\hline \multirow{2}{*}{$\begin{array}{l}\text { Traverse--> } \\
\text { Trial } \longrightarrow\end{array}$} & & \multicolumn{4}{|c|}{ East } & \multicolumn{4}{|c|}{ North } \\
\hline & & 1 & 2 & 3 & Mean & 1 & 2 & 3 & Mean \\
\hline Point & Depth, in. & \multicolumn{4}{|c|}{$\mathrm{ppm}$} & \multicolumn{4}{|c|}{$\mathrm{ppm}$} \\
\hline 1 & 0.58 & 3.10 & 3.67 & 3.19 & 3.32 & 3.68 & 3.73 & 3.94 & 3.78 \\
\hline 2 & 1.89 & 3.14 & 3.51 & 3.37 & 3.34 & 3.54 & 3.39 & 3.68 & 3.54 \\
\hline 3 & 3.49 & 3.31 & 3.30 & 4.08 & 3.56 & 3.44 & 3.85 & 3.50 & 3.60 \\
\hline 4 & 5.81 & 3.32 & 4.17 & 3.63 & 3.71 & 3.50 & 3.79 & 3.69 & 3.66 \\
\hline Center & 9.00 & 3.66 & 4.02 & 3.96 & 3.88 & 3.73 & 3.52 & 3.38 & 3.54 \\
\hline 5 & 12.19 & 4.17 & 3.69 & 3.62 & 3.83 & 3.58 & 3.23 & 3.54 & 3.45 \\
\hline 6 & 14.51 & 3.42 & 3.43 & 3.78 & 3.54 & 3.26 & 3.78 & 3.81 & 3.62 \\
\hline 7 & 16.11 & 3.65 & 3.47 & 3.48 & 3.53 & 3.43 & 3.83 & 3.43 & 3.56 \\
\hline 8 & 17.42 & $\overline{3.92}$ & 3.02 & 3.62 & 3.52 & 3.45 & 3.59 & 3.86 & 3.63 \\
\hline Averages - & $\Longrightarrow$ & 3.52 & 3.59 & 3.64 & 3.58 & 3.51 & 3.63 & 3.65 & 3.60 \\
\hline
\end{tabular}

\begin{tabular}{|llr|lllr|}
\hline All & ppm & Dev. from mean & Center 2/3 & East & North & All \\
Mean & 3.59 & & Mean & 3.63 & 3.57 & 3.60 \\
Min Point & 3.32 & $-7.5 \%$ & Std. Dev. & 0.19 & 0.07 & 0.14 \\
Max Point & 3.88 & $8.1 \%$ & COV as $\%$ & 5.19 & 1.89 & 3.88 \\
\hline
\end{tabular}

Avg. Conc. $\quad 3.575 \mathrm{ppm}$

Tracer tank pressure Sample Port Temp Centerline vel. Injection flowmeter Ambient temp. Sampling flowmeter Ambient pressure Ambient humidity B\&K vapor correction

Back-Gd gas level No. Bk-Gd samples Ambient pressure Instuments Used: B \& K Model 1302\#1765299 Sierra Inc. Constant Flow Air Sampler Solomat Zephyr SN 1295-1472

${ }^{* *}$ Reading on black plastic ball float in flowmeter Notes: At this point, the duct is $18 \times 18$ inches The injection probe reaches 8 -in off center, so with the elevation adjusted accordingly, the injection is one-inch from both sides of a corner. Also, the center injection point is 8-in closer to the stack than the injection port. Signature signifies compliance with Procedure EMS-JAG-01 Signature/date

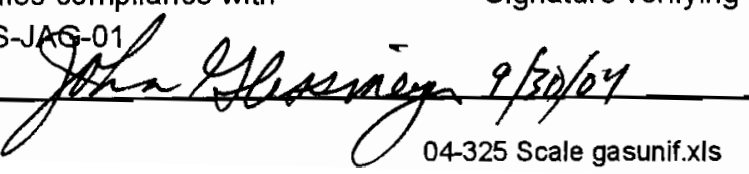
04-325 Scale gasunif.xis

\begin{tabular}{|c|c|c|}
\hline Start & Finish & \\
\hline 310 & 330 & psig \\
\hline 84.9 & 84.7 & $F$ \\
\hline 782 & 801.0 & fpm \\
\hline 4 & 4 & $\%$ bl. ball* \\
\hline 78 & 80 & $\operatorname{deg} . F$ \\
\hline 10 & 10 & lipm Sierra \\
\hline 751.20 & 750.5 & $\mathrm{~mm} \mathrm{Hg}$ \\
\hline 30 & 27 & $\% R H$ \\
\hline $\mathrm{N}$ & $N$ & $\mathrm{Y} / \mathrm{N}$ \\
\hline $29 / 23 / 31 / 29 /$ & $29 / 28 / 22 / 21 /$ & \\
\hline $26 / 31$ & 25 & $p p b$ \\
\hline 6 & 5 & $n$ \\
\hline 1016.5 & 1015.6 & mbar \\
\hline
\end{tabular}

Gas analyzer checked:

$9 / 1 / 2004$

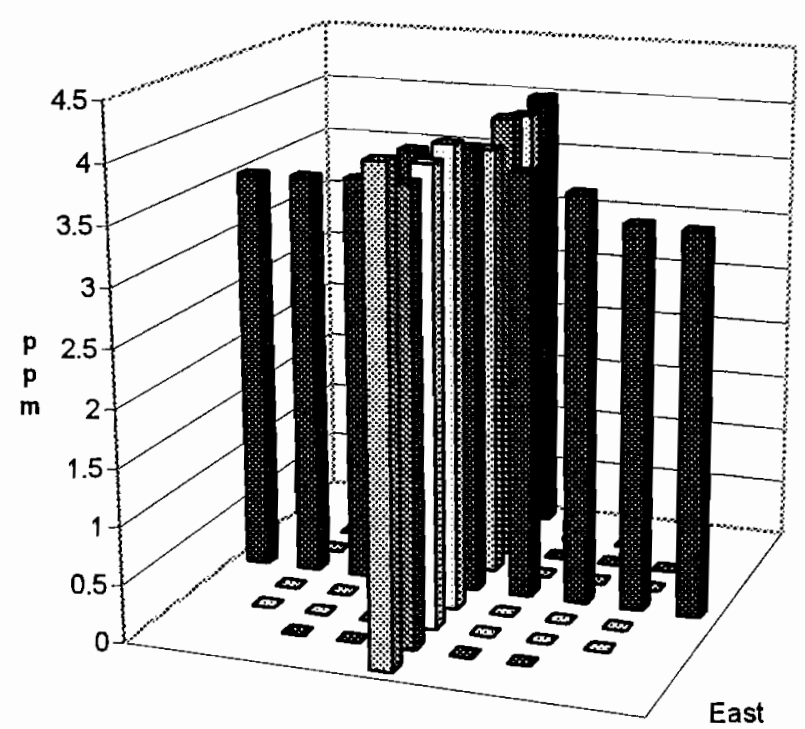

North

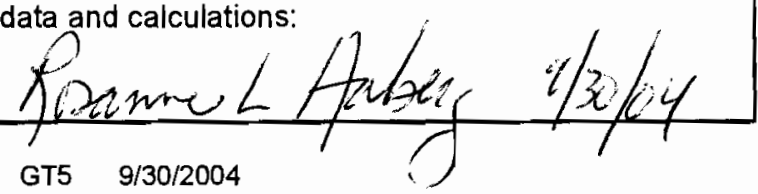


TRACER GAS TRAVERSE DATA FORM

Site 325 Model

Date 9/3/2004

Tester JAG/JMB

Stack Dia.

Stack X-Area

Elevation N.A.

Distance to disturbance

Measurement units ppm SFG
Run No. GT-6

Fan Configuration Far fan

Fan Setting $37.1 \mathrm{~Hz}$

Stack Temp $88.25 \mathrm{deg} F$

Start/End Time 1504/1630

Center $2 / 3$ from 1.65 to: 16.35

Points in Center 2/3 2 to: 7

Injection Point Top South, 1-inch from corner walls

\begin{tabular}{|c|c|c|c|c|c|c|c|c|c|}
\hline \multirow{2}{*}{$\begin{array}{l}\text { Traverse--> } \\
\text { Trial --> }\end{array}$} & & \multicolumn{4}{|c|}{ East } & \multicolumn{4}{|c|}{ North } \\
\hline & & 1 & 2 & 3 & Mean & 1 & 2 & 3 & Mean \\
\hline Point & Depth, in. & \multicolumn{4}{|c|}{$\mathrm{ppm}$} & \multicolumn{4}{|c|}{$\mathrm{ppm}$} \\
\hline 1 & 0.58 & 4.01 & 3.89 & 4.03 & 3.98 & 3.98 & 3.84 & 3.94 & 3.92 \\
\hline 2 & 1.89 & $3 . \overline{79}$ & 3.91 & 3.83 & 3.84 & 3.80 & 3.76 & 4.06 & 3.87 \\
\hline 3 & 3.49 & 3.97 & 3.96 & 4.02 & 3.98 & 3.83 & 4.04 & 3.82 & 3.90 \\
\hline 4 & 5.81 & 3.83 & 3.92 & 3.87 & 3.87 & 3.87 & 3.72 & 3.85 & 3.81 \\
\hline Center & 9.00 & 3.93 & 3.73 & 4.01 & 3.89 & 3.85 & 4.13 & 3.91 & 3.96 \\
\hline 5 & 12.19 & 3.74 & 4.06 & 4.16 & 3.99 & $3 . \overline{77}$ & 4.05 & 4.09 & 3.97 \\
\hline 6 & 14.51 & 3.99 & 3.88 & 3.89 & 3.92 & 3.82 & 3.79 & 3.90 & 3.84 \\
\hline 7 & 16.11 & 3.95 & 3.99 & 3.97 & 3.97 & 3.89 & 4.00 & 4.14 & 4.01 \\
\hline 8 & 17.42 & 3.75 & 3.80 & 3.99 & 3.85 & 3.92 & $3 . \overline{95}$ & 3.75 & 3.87 \\
\hline Averages -- & $\longrightarrow$ & 3.88 & 3.90 & 3.97 & 3.92 & 3.86 & 3.92 & 3.94 & 3.91 \\
\hline
\end{tabular}

Avg. Conc. $\quad 3.912 \mathrm{ppm}$

\begin{tabular}{|lc}
\hline AlI & $\mathrm{ppm}$ \\
Mean & 3.91 \\
Min Point & 3.8 \\
Max Point & 4.01 \\
\hline
\end{tabular}

Tracer tank pressure Sample Port Temp Centerline vel. Injection flowmeter Ambient temp. Sampling flowmeter Ambient pressure Ambient humidity B\&K vapor correction

Back-Gd gas level No. Bk-Gd samples Ambient pressure Instuments Used: B \& K Model $1302 \# 1765299$

Sierra inc. Constant Flow Air Sampler

Solomat Zephyr SN 1295-1472

${ }^{* \star}$ Reading on black plastic ball float in flowmeter Notes: At this point, the duct is $11 \times 12$ inches The injection probe reaches 5 -in off center, so with the elevation adjusted accordingly, the injection is one-inch from both sides of a corner. Also, the center injection point is 5-in closer to the stack than the injection port.

\begin{tabular}{|c|c|c|c|c|}
\hline Dev from mean & Center 2/3 & East & North & All \\
\hline & Mean & 3.92 & 3.91 & 3.92 \\
\hline$-2.6 \%$ & Std. Dev. & 0.06 & 0.07 & 0.06 \\
\hline $2.5 \%$ & $\operatorname{Cov}$ as $\%$ & 1.46 & 1.89 & 1.63 \\
\hline
\end{tabular}

Gas analyzer checked: $9 / 1 / 2004$

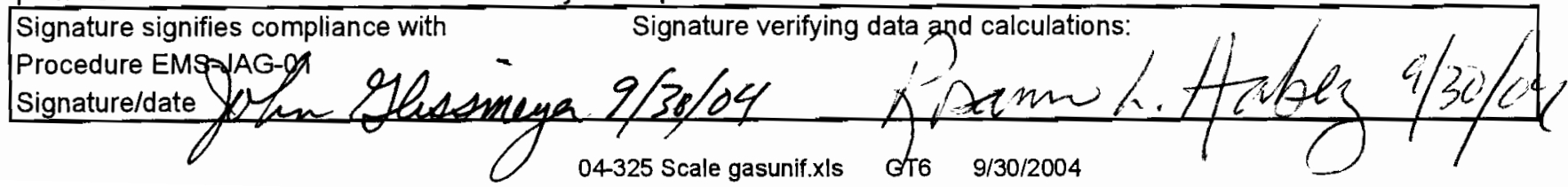


TRACER GAS TRAVERSE DATA FORM

Site 325 Model

Date 9/8/2004

Tester JAG/JMB

Stack Dia.

18 in.

Stack X-Area 254.5 in.2

Elevation N.A

Distance to disturbance 120 inches

Measurement units ppm SF6
Run No. GT-7

Fan Configuration Far fan

Fan Setting $37.1 \mathrm{~Hz}$

Stack Temp $84 \operatorname{deg} F$

Start/End Time $1130 / 1222$

Center $2 / 3$ from $\quad 1.65$ to: 16.35

Points in Center $2 / 3=2$ to: 7 Injection Point Top North, 1-inch from corner walls

\begin{tabular}{|c|c|c|c|c|c|c|c|c|c|}
\hline \multirow{2}{*}{$\begin{array}{l}\text { Traverse-> } \\
\text { Trial -_> }\end{array}$} & & \multicolumn{4}{|c|}{ East } & \multicolumn{4}{|c|}{ North } \\
\hline & & 1 & 2 & 3 & Mean & 1 & 2 & 3 & Mean \\
\hline Point & Depth, in. & \multicolumn{4}{|c|}{$\mathrm{ppm}$} & \multicolumn{4}{|c|}{$\mathrm{ppm}$} \\
\hline 1 & 0.58 & 3.33 & 3.32 & 3.58 & 3.41 & 3.36 & 3.35 & 3.40 & 3.37 \\
\hline 2 & 1.89 & 3.27 & 3.32 & 3.24 & 3.28 & 3.38 & 3.25 & 3.33 & 3.32 \\
\hline 3 & 3.49 & 3.23 & 3.43 & 3.41 & 3.36 & 3.48 & 3.39 & 3.31 & 3.39 \\
\hline 4 & 5.81 & 3.26 & 3.36 & 3.59 & 3.40 & 3.51 & 3.19 & 3.21 & 3.30 \\
\hline Center & $9 . \overline{00}$ & 3.42 & 3.42 & 3.46 & 3.43 & 3.19 & 3.24 & 3.48 & 3.30 \\
\hline 5 & 12.19 & 3.22 & 3.41 & 3.42 & 3.35 & 3.44 & 3.36 & 3.58 & 3.46 \\
\hline 6 & 14.51 & 3.49 & 3.35 & 3.23 & 3.36 & 3.44 & 3.53 & 3.45 & 3.47 \\
\hline 7 & 16.11 & 3.39 & 3.18 & 3.37 & 3.31 & 3.39 & 3.26 & 3.36 & 3.34 \\
\hline 8 & 17.42 & 3.30 & 3.15 & 3.35 & 3.27 & 3.55 & 3.28 & 3.26 & 3.36 \\
\hline Averages - & $\rightarrow$ & 3.32 & 3.33 & 3.41 & 3.35 & 3.42 & 3.32 & 3.38 & 3.37 \\
\hline
\end{tabular}

Avg. Conc. $\quad 3.360 \mathrm{ppm}$

\begin{tabular}{|lc}
\hline All & $\mathrm{ppm}$ \\
Mean & 3.36 \\
Min Point & 3.27 \\
Max Point & 3.47 \\
\hline
\end{tabular}

Tracer tank pressure Sample Port Temp Centerline vel. Injection flowmeter Ambient temp. Sampling flowmeter Ambient pressure Ambient humidity B\&K vapor correction

Back-Gd gas leve! No. Bk-Gd samples Ambient pressure Instuments Used: B \& K Model $1302 \# 1765299$

Sierra Inc. Constant Flow Air Sampler

Solomat Zephyr SN 1295-1472

**Reading on black plastic ball float in flowmeter Notes: At this point, the duct is $11 \times 12$ inches The injection probe reaches 5 -in off center, so with the elevation adjusted accordingly, the injection is one-inch from both sides of a corner. Also, the center injection point is 5-in closer to the stack than the injection port.
Signature signifies compliance with Signature verifying data and calculations:

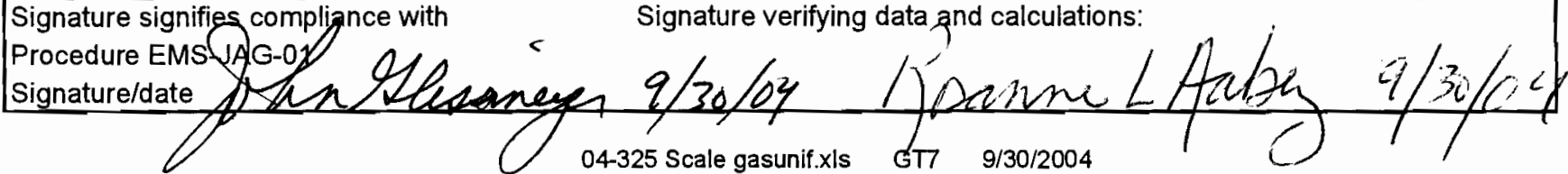


TRACER GAS TRAVERSE DATA FORM

Site 325 Model

Date .9/8/2004

Tester JAG/JMB

Stack Dia

18 in.

Stack X-Area 254.5 in. 2

Elevation N.A.

Distance to disturbance 120 inches

Measurement units ppm SF6
Run No. GT-8

Fan Configuration Far fan

Fan Setting $37.1 \mathrm{~Hz}$

Stack Temp

Center $2 / 3$ from $\quad 1.65$ to: 16.35

Points in Center $2 / 3 \longdiv { 2 }$ to: $\overline{7}$

Injection Point Center

\begin{tabular}{l}
\begin{tabular}{l} 
Traverse-- \\
\cline { 2 - 9 }
\end{tabular} \\
\begin{tabular}{|r|r|r|r|r|r|r|} 
Trial $\rightarrow$ \\
\hline Point
\end{tabular} \\
\hline 1
\end{tabular}

Avg. Conc. $\quad 3.382 \mathrm{ppm}$

Tracer tank pressure Sample Port Temp Centerline vel. Injection flowmeter Ambient temp. Sampling flowmeter Ambient pressure Ambient humidity B\&K vapor correction

Back-Gd gas level No. Bk-Gd samples Ambient pressure Instuments Used: B \& K Model $1302 \# 1765299$

Sierra inc. Constant Flow Air Sampler

Solomat Zephyr SN 1295-1472

${ }^{\star \star}$ Reading on black plastic ball float in flowmeter

Notes: At this point, the duct is $11 \times 12$ inches The injection probe reaches 5 -in off center, so with the elevation adjusted accordingly, the injection is one-inch from both sides of a corner. Also, the center injection point is 5 -in closer to the stack than the injection port.
Signature signifies compliance with

Procedure EMS-JAG-01

Signature/date

\begin{tabular}{|lc}
\hline All & ppm \\
Mean & 3.38 \\
Min Point & 3.24 \\
Max Point & 3.54 \\
\hline
\end{tabular}

\begin{tabular}{|c|c|c|}
\hline Start & Finish & \\
\hline 315 & 370 & psig \\
\hline 85 & 89 & $\mathrm{~F}$ \\
\hline 765 & 739.0 & $\mathrm{fpm}$ \\
\hline 3.5 & 3.5 & $\%$ bl. ba \\
\hline 79 & 80 & deg. $F$ \\
\hline 10 & 9.5 & ipm Sier \\
\hline 748.30 & 747.8 & $\mathrm{~mm} \mathrm{Hg}$ \\
\hline 35 & 32 & $\% R H$ \\
\hline$N$ & $\mathrm{~N}$ & $Y / N$ \\
\hline $38 / 37 / 36 / 35$ & $32 / 32 / 35 / 31 /$ & \\
\hline 35 & 31 & $\mathrm{ppb}$ \\
\hline 5 & 5 & n \\
\hline 1012.9 & 1012.2 & mbar \\
\hline
\end{tabular}

\begin{tabular}{r|l} 
Dev. from mean & Center 2/3 \\
$-4.1 \%$ & Std. Dean \\
$4.8 \%$ & COV as $\%$
\end{tabular}

Gas analyzer checked:

$9 / 1 / 2004$
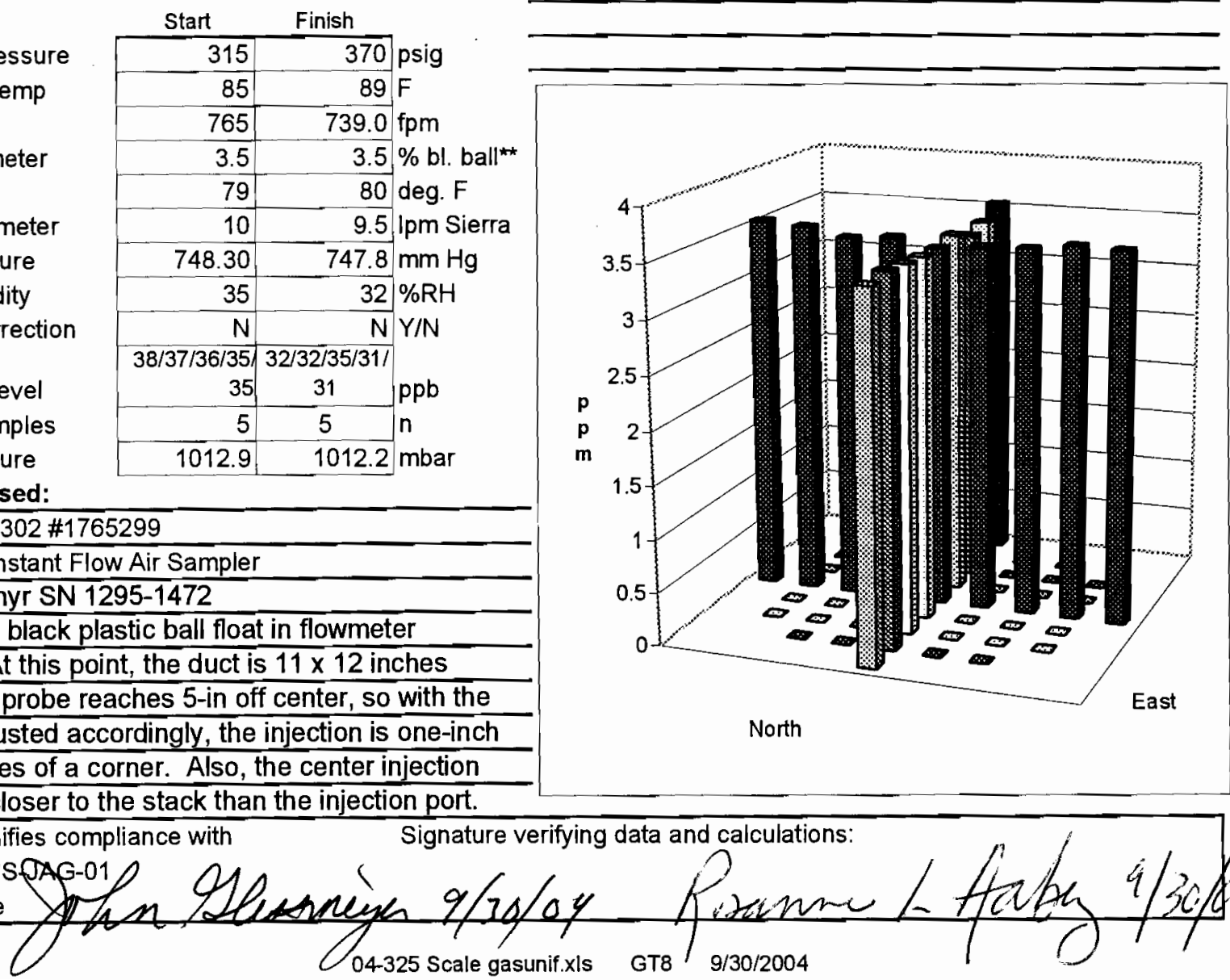

\begin{tabular}{lll|}
$\frac{\text { East }}{3.30}$ & $\frac{\text { North }}{3.43}$ & All \\
0.04 & 0.03 & 0.08 \\
1.20 & 0.89 & $\mathbf{2 . 2 8}$ \\
\hline
\end{tabular}

Signature verifying data and calculations: 
TRACER GAS TRAVERSE DATA FORM

Site 325 Model
Date $\frac{9 / 8 / 2004}{18 \text { in. }}$
Tester JMB
Stack Dia. $\frac{18 \text { in. }}{254.5}$
Stack X-Area
Elevation N.A.
Distance to disturbance
Measurement units ppm SF6

Run No. GT-9

Fan Configuration Far fan

Fan Setting $37.1 \mathrm{~Hz}$

Stack Temp 89.5 deg $F$

Start/End Time $1302 / 1354$

Center $2 / 3$ from 1.65 to: 16.35

Points in Center $2 / 3 \overline{2}$ to: $\overline{7}$ Injection Point Bottom North

\begin{tabular}{|c|c|c|c|c|c|c|c|c|c|}
\hline \multirow{2}{*}{$\begin{array}{l}\text { Traverse--> } \\
\text { Trial -_-> }\end{array}$} & & \multicolumn{4}{|c|}{ East } & \multicolumn{4}{|c|}{ North } \\
\hline & & 1 & 2 & 3 & Mean & 1 & 2 & 3 & Mean \\
\hline Point & Depth, in. & \multicolumn{4}{|c|}{$\mathrm{ppm}$} & \multicolumn{4}{|c|}{$\mathrm{ppm}$} \\
\hline 1 & 0.58 & 3.24 & 3.54 & 3.21 & 3.33 & 3.37 & 3.26 & 2.98 & 3.20 \\
\hline 2 & 1.89 & 3.49 & 3.33 & 3.50 & 3.44 & 3.25 & 3.36 & 3.20 & 3.27 \\
\hline 3 & 3.49 & 3.27 & 3.23 & 3.35 & 3.28 & 3.47 & 3.33 & 3.23 & 3.34 \\
\hline 4 & 5.81 & 3.58 & 3.35 & 3.40 & 3.44 & 3.42 & 3.09 & 3.18 & 3.23 \\
\hline Center & 9.00 & 3.28 & 3.26 & 3.46 & 3.33 & 3.61 & 3.41 & 3.33 & 3.45 \\
\hline 5 & 12.19 & 3.70 & 3.36 & 3.13 & 3.40 & 3.72 & 3.12 & 3.17 & 3.34 \\
\hline 6 & 14.51 & 3.46 & 3.38 & 3.34 & 3.39 & 3.76 & 3.34 & 3.32 & 3.47 \\
\hline 7 & 16.11 & 3.27 & 3.27 & 3.22 & 3.25 & 3.64 & 3.36 & 3.41 & 3.47 \\
\hline 8 & $17 . \overline{42}$ & 3.36 & 3.27 & 3.42 & 3.35 & 3.43 & 3.23 & 3.35 & 3.34 \\
\hline \multirow{5}{*}{\multicolumn{2}{|c|}{ Averages $\ldots$}} & 3.41 & 3.33 & 3.34 & 3.36 & 3.52 & 3.28 & 3.24 & 3.35 \\
\hline & & $A \|$ & $\mathrm{ppm}$ & Dev & from mean & Center 2/3 & East & North & All \\
\hline & & Mean & 3.35 & & & Mean & 3.36 & 3.37 & 3.37 \\
\hline & & Min Point & 3.20 & & $-4.4 \%$ & Std. Dev. & 0.07 & 0.10 & 0.08 \\
\hline & & Max Point & 3.47 & & $3.6 \%$ & COV as $\%$ & 2.23 & 2.93 & 2.50 \\
\hline
\end{tabular}

Avg. Conc. $\quad 3.347 \mathrm{ppm}$

Tracer tank pressure Sample Port Temp Centerline vel. Injection flowmeter Ambient temp. Sampling flowmeter Ambient pressure Ambient humidity $B \& K$ vapor correction

Back-Gd gas level No. Bk-Gd samples Ambient pressure Instuments Used: B \& K Model 1302 \#1765299

Sierra inc. Constant Flow Air Sampler

Solomat Zephyr SN 1295-1472

** Reading on black plastic ball float in flowmeter

Notes: At this point, the duct is $11 \times 12$ inches The injection probe reaches 5 -in off center, so with the elevation adjusted accordingly, the injection is one-inch from both sides of a corner. Also, the center injection point is 5-in closer to the stack than the injection port.

\section{Gas analyzer checked:} $9 / 1 / 2004$

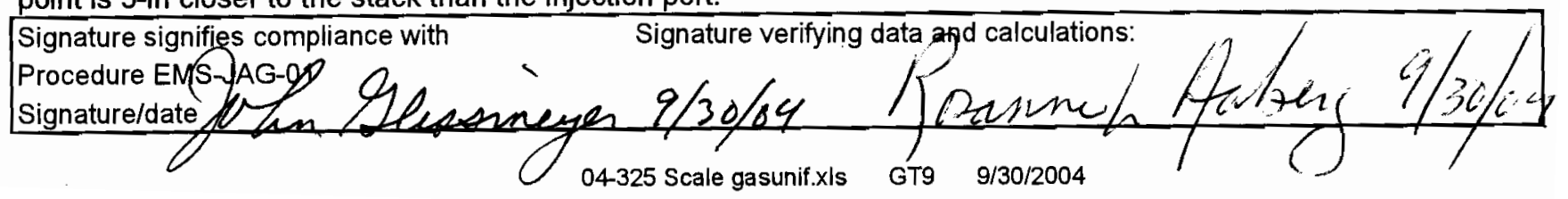


TRACER GAS TRAVERSE DATA FORM

Site 325 Model

Date 9/8/2004

Tester JMB/JAG

Stack Dia.

Stack X-Area

Elevation N.A

Distance to disturbance

Measurement units ppm SFG
Run No. GT-10

Fan Configuration Far fan

Fan Setting $37.1 \mathrm{~Hz}$

Stack Temp

Start/End Time $\overline{1354 / 1434}$

Center $2 / 3$ from 1.65 to: 16.35

Points in Center $2 / 3$

Injection Point Bottom South

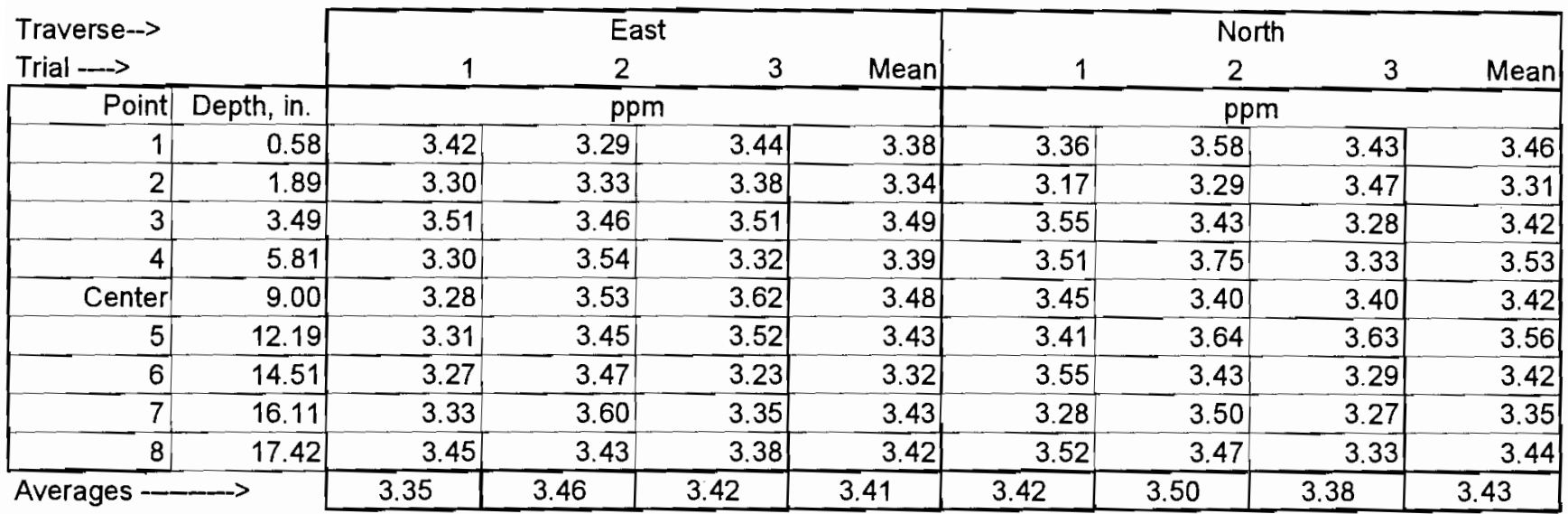

Avg. Conc. $\quad 3.418 \mathrm{ppm}$

\begin{tabular}{ll}
\hline All & $\mathrm{ppm}$ \\
Mean & 3.42 \\
Min Point & 3.31 \\
Max Point & 3.56 \\
\hline
\end{tabular}

\begin{tabular}{|c|c|c|c|}
\hline & Start & Finish & \\
\hline Tracer tank pressure & 390 & 390 & Jpsig \\
\hline Sample Port Temp & 92 & 92 & $F$ \\
\hline Centerline vel. & 764.0 & 763.0 & $\mathrm{fpm}$ \\
\hline injection flowmeter & 3.5 & 3.5 & $\%$ bi. ball* \\
\hline Ambient temp. & 83 & 85 & deg. F \\
\hline Sampling flowmeter & 10 & 10 & Ipm Sierra \\
\hline Ambient pressure & 747.4 & 747.1 & $\mathrm{~mm} \mathrm{Hg}$ \\
\hline Ambient humidity & 29 & 27 & $\% \mathrm{RH}$ \\
\hline B\&K vapor correction & $\mathrm{N}$ & $N$ & $Y / N$ \\
\hline & $38 / 33 / 31 / 32 /$ & $46 / 31 / 36 / 381$ & \\
\hline Back-Gd gas levei & 30 & 30 & $\mathrm{ppb}$ \\
\hline No. Bk-Gd samples & 5 & 5 & $n$ \\
\hline Ambient pressure & 1012 & 1012 & mbar \\
\hline
\end{tabular}

Instuments Used:

B \& K Model $1302 \# 1765299$

Sierra Inc. Constant Flow Air Sampler

Solomat Zephyr SN 1295-1472

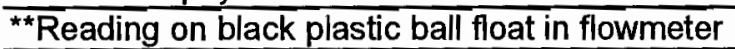

Notes: At this point, the duct is $11 \times 12$ inches

The injection probe reaches 5 -in off center, so with the

elevation adjusted accordingly, the injection is one-inch

from both sides of a corner. Also, the center injection

point is 5-in closer to the stack than the injection port.

\begin{tabular}{r|llll|}
\hline Dev. from mean & Center 2/3 & East & North & Ail \\
$-3.2 \%$ & Mean & 3.41 & 3.43 & 3.42 \\
$4.1 \%$ & Std. Dev. & 0.07 & 0.09 & 0.08 \\
\hline
\end{tabular}

Gas analyzer checked:

$9 / 1 / 2004$

Signature signifies compliance with Signature verifying data andfcalculations:

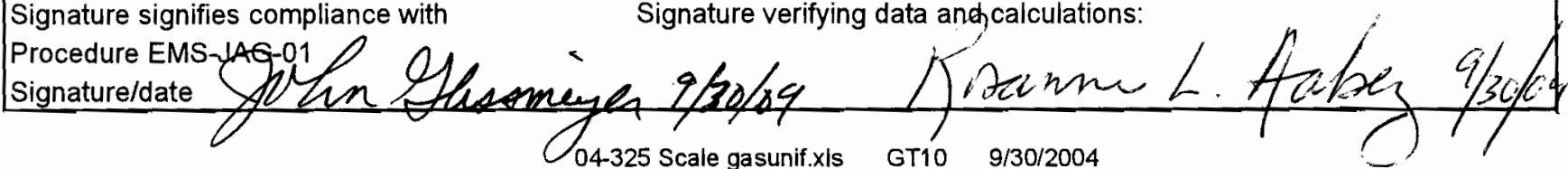


TRACER GAS TRAVERSE DATA FORM

\begin{tabular}{|c|c|}
\hline \multicolumn{2}{|c|}{ Site 325 Model } \\
\hline Date & $9 / 8 / 2004$ \\
\hline \multicolumn{2}{|c|}{ Tester JMB/JAG } \\
\hline Stack Dia. & $18 \mathrm{in}$. \\
\hline Stack X-Area & 254.5 in 2 \\
\hline \multicolumn{2}{|c|}{ Elevation N.A. } \\
\hline Distance to disturbance & 120 inches \\
\hline
\end{tabular}

Run No. GT-11

Fan Configuration Near fan

Fan Setting $37.1 \mathrm{~Hz}$

Stack Temp $90 \mathrm{deg} F$

Start/End Time $1505-1545$

Center $2 / 3$ from 1.65 to: 16.35

Points in Center $2 / 3 \longdiv { 2 }$ to: $\overline{7}$

Injection Point Top South

\begin{tabular}{|c|c|c|c|c|c|c|c|c|c|}
\hline \multirow{2}{*}{\multicolumn{2}{|c|}{$\begin{array}{l}\text { Traverse--> } \\
\text { Trial -.-> }\end{array}$}} & \multicolumn{4}{|c|}{ East } & \multicolumn{4}{|c|}{ North } \\
\hline & & 1 & 2 & 3 & Mean & 1 & 2 & 3 & Mean \\
\hline Point & Depth, in. & \multicolumn{4}{|c|}{$\mathrm{ppm}$} & \multicolumn{4}{|c|}{ ppm } \\
\hline 1 & 0.58 & 3.32 & 3.80 & 3.32 & 3.48 & 3.02 & 2.65 & 3.62 & 3.10 \\
\hline 2 & 1.89 & 4.00 & 3.45 & 3.44 & 3.63 & 3.03 & 3.37 & 3.58 & 3.33 \\
\hline 3 & 3.49 & 3.76 & 3.42 & 3.47 & 3.55 & $3 . \overline{21}$ & 3.31 & 3.49 & 3.34 \\
\hline 4 & 5.81 & 3.62 & 3.18 & 3.78 & 3.53 & $3 . \overline{47}$ & 3.50 & 3.35 & 3.44 \\
\hline Center & 9.00 & 3.28 & 3.45 & 3.11 & 3.28 & 3.38 & 3.42 & 3.65 & 3.48 \\
\hline 5 & 12.19 & 3.03 & 2.99 & 2.99 & 3.00 & 3.07 & 3.48 & 3.46 & 3.34 \\
\hline 6 & 14.51 & 3.45 & 3.08 & 3.61 & 3.38 & 3.31 & 3.50 & 3.08 & 3.30 \\
\hline 7 & 16.11 & 3.26 & 3.46 & 3.46 & 3.39 & 2.86 & 3.14 & 2.96 & 2.99 \\
\hline 8 & 17.42 & 3.14 & 3.42 & 3.22 & 3.26 & 2.77 & 3.04 & 3.30 & 3.04 \\
\hline Averages - & $\rightarrow$ & 3.43 & 3.36 & 3.38 & 3.39 & 3.12 & 3.27 & 3.39 & 3.26 \\
\hline
\end{tabular}

\begin{tabular}{|c|c|c|c|c|c|c|}
\hline$\overline{A l l}$ & ppm & Dev. from mean & Center 2/3 & East & North & All \\
\hline Mean & 3.32 & & Mean & 3.39 & 3.32 & $3 . \overline{36}$ \\
\hline Min Point & 2.99 & $-10.2 \%$ & Std. Dev. & 0.21 & 0.16 & 0.18 \\
\hline Max Point & 3.63 & $9.2 \%$ & COV as $\%$ & 6.17 & 4.82 & 5.48 \\
\hline
\end{tabular}

Avg. Conc. $\quad 3.318 \mathrm{ppm}$

Gas analyzer checked:

$9 / 1 / 2004$

Tracer tank pressure

Sample Port Temp

Centerline vel.

Injection flowmeter

Ambient temp.

Sampling flowmeter

Ambient pressure

Ambient humidity

B\&K vapor correction

Back-Gd gas level

No, Bk-Gd samples

Ambient pressure

Instuments Used:

\begin{tabular}{|c|c|c|}
\hline Start & Finish & \\
\hline 350 & 390 & psig \\
\hline 89 & 91 & $F$ \\
\hline 769.0 & 768.0 & $\mathrm{fpm}$ \\
\hline 3.5 & 3.5 & $\%$ bl ball $\|^{\star \star *}$ \\
\hline 85 & 89 & deg. F \\
\hline 10 & 10 & Ipm Sierra \\
\hline 747.1 & 746.4 & $\mathrm{~mm} \mathrm{Hg}$ \\
\hline 27 & 27 & $\% R H$ \\
\hline $\mathrm{N}$ & $\mathrm{N}$ & $Y / N$ \\
\hline $\begin{array}{c}46 / 31 / 36 / 381 \\
30\end{array}$ & $\begin{array}{c}36 / 34 / 36 / 30 / \\
30\end{array}$ & $\mathrm{ppb}$ \\
\hline 5 & 5 & $n$ \\
\hline 1012 & 1011 & mbar \\
\hline
\end{tabular}

B \& K Model 1302 \#1765299

Sierra Inc. Constant Flow Air Sampler

Solomat Zephyr SN 1295-1472

* Reading on black plastic ball float in flowmeter

Notes:
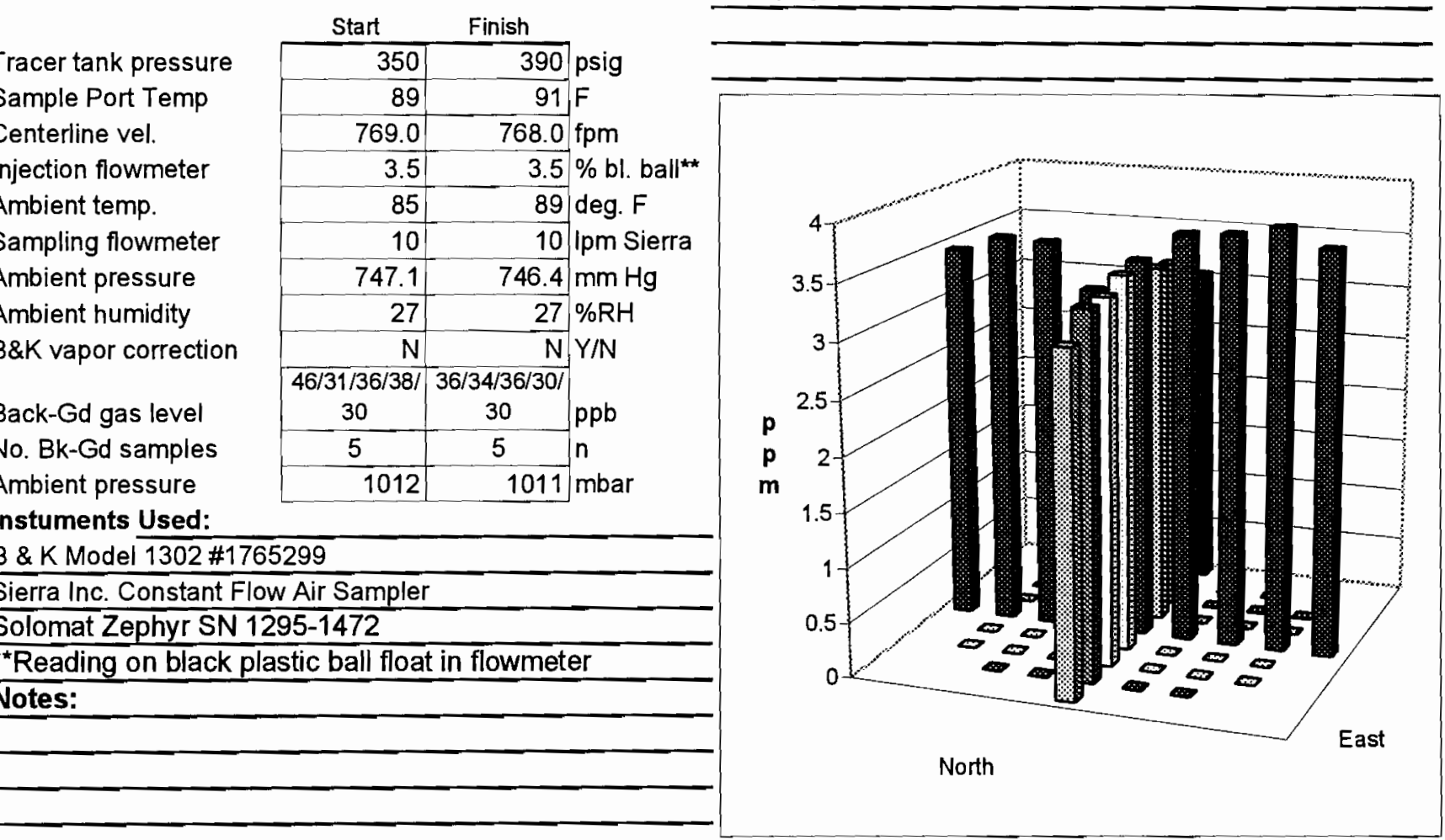

Signature signifies compliance with

Signature verifying data and calcylations:

Procedure EMS-\$AG-010

Signature/date

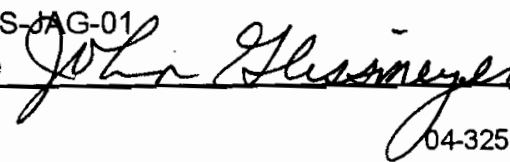

4-325 scale gasunif.xls

GT11

$9 / 30 / 2004$

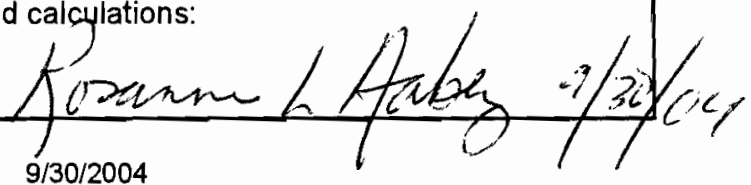




\begin{tabular}{|c|c|c|c|}
\hline \multicolumn{4}{|c|}{ Test Instruction } \\
\hline \multicolumn{2}{|c|}{$\begin{array}{l}\text { Project: } 325 \text { Stack Sampler } \\
\text { Qualification }\end{array}$} & & Work Package: F59676 \\
\hline \multicolumn{4}{|c|}{ Tests: Tracer Gas Uniformity in 325 Model Stack, 1 Electric Fan Configuration } \\
\hline \multicolumn{4}{|c|}{ Staff: John Glissmeyer, Dave Douglas, Marcel Ballinger, Matthew Barnett } \\
\hline \multicolumn{4}{|c|}{$\begin{array}{l}\text { Reference Procedures: } \\
\text { 1. Procedure EMS-JAG-01, Rev. 1, Test to Determine Uniformity of a Tracer Gas at a Sampler } \\
\text { Probe, May } 26,2000 \\
\text { 2. Operating Manual for Bruel and Kjaer Model } 1302 \text { Gas Analyzer }\end{array}$} \\
\hline \multicolumn{4}{|c|}{$\begin{array}{l}\text { Equipment: } \\
\text { 1. } 325 \text { Model Stack and inspected work platforms. Fans will be in positions EF1, EF4. } \\
\text { 2. Sulfur hexafluoride gas (pure and calibration gas), regulator, control valve, rotameter, injection } \\
\text { probe, and tubing. Injection occurs in ports along horizontal duct. The near wall injection points } \\
\text { are within } 3 \text { in. ( } 25 \% \text { of hydraulic diameter) for smaller duct section and } 4 \text { in. for } 18 \text { " x } 18 \text { " duct. } \\
\text { Size injection probe accordingly. } \\
\text { 3. Bruel and Kjaer Model } 1302 \text { Gas Analyzer, probe, vacuum pump, fittings }\end{array}$} \\
\hline \multicolumn{4}{|c|}{$\begin{array}{l}\text { Safety Considerations: } \\
\text { Observe the applicable Job Hazard Analysis for the project }\end{array}$} \\
\hline \multicolumn{4}{|c|}{$\begin{array}{l}\text { Instructions: } \\
\text { 1. Verify training on the procedure and verify that instrumentation is within calibration } \\
\text { 2. Weigh the tracer cylinder before shipment to jobsite } N \text {. no seale avalable } 9 / 1 \% 4 \\
\text { 3. Obtain climatic information from the Hanford Weather Service, phone } 373-2716 \text { or } \\
\text { http://etd.pnl.gov:2080/HMS/lastob.htm } \\
\text { 4. Mark the completion of each step on the field copy of the procedure. Mark-out those steps not } \\
\text { applicable to this stack. } \\
\text { 5. Install equipment as directed in the procedures } \\
\text { 6. Mark sampling probe for the measurement points shown on the data sheet } \\
\text { 7. With one fan operating, verify that stack flow is about } 1350-1450 \mathrm{cfm} . \\
\text { 8. Set the injection flowrate at about } 0.07 \text { lpm for a tracer concentration of } \sim 1 \mathrm{ppm} \text { (reads about } 0.14 \\
\text { lpm on an air rotameter when corrected for gas density, about } 7 \text { on the rotameter scale with the } \\
\text { glass ball). } \\
\text { 9. Set the sampler flowrate at approximately } 10 \text { lpm. } \\
\text { 10. Record each run's data on copies of the attached data sheet } \\
\text { 12. Diagram mounting fixtures and retain assembly for any subsequent re-tests } \\
\text { 12. Conduct one or more tracer mixing tests at the following sets of conditions: }\end{array}$} \\
\hline $\begin{array}{l}\text { Downstream } \\
\text { of Fan }\end{array}$ & Injection Pos & ions & Possible Runs \\
\hline EF4ON & Center, 1 inc & om each corner & $\mathrm{GT} 1-5$ \\
\hline $\mathrm{EF} 1 \mathrm{ON}$ & Center, 1 inc & & GT $6-10$ \\
\hline Worst case & Repeat of wc & st case & GT 11 \\
\hline \multicolumn{4}{|c|}{ Desired Completion Date: $08 / 27 / 04$} \\
\hline \multicolumn{3}{|c|}{ Approvals: $\frac{\text { Joh }}{\text { Jolh Glissmeyer, test director }}$} & $\frac{8725 / 04}{\text { Date }}$ \\
\hline \multicolumn{4}{|c|}{ Test completed by: Ylesomege $\quad$ Date: $9 / 8 / 04$} \\
\hline
\end{tabular}




\begin{tabular}{l|ll}
\hline PNNL Operating Procedure & $\begin{array}{l}\text { Rev. No. 1 } \\
\text { Org. Code: D9T99 }\end{array}$ & $\begin{array}{l}\text { Page 1 of 16 } \\
\text { Procedure No.: EMS-JAG-01 }\end{array}$ \\
\hline
\end{tabular}

Title: Test to Determine Uniformity of a Tracer Gas at a Sampler Probe

PNNL Operating Procedure

Title: Test to Determine

Uniformity of a Tracer

Gas at a Sampler Probe

\begin{tabular}{|c|c|c|c|}
\hline Gas al a waimpier i ruye & \multicolumn{3}{|c|}{ Rev. No.: $\quad 1$} \\
\hline Work Location: General & Effective Date: & \multicolumn{2}{|c|}{ May 26, 2000} \\
\hline Author: John A. Glissmeyer & Supersedes Date: & \multicolumn{2}{|c|}{ November 10, 1998} \\
\hline $\begin{array}{l}\text { Identified Hazards: } \\
\square \text { Radiological } \\
\square \text { Hazardous Materials } \\
\text { Physical Hazards } \\
\square \text { Hazardous Environment } \\
\square \text { Other: }\end{array}$ & \multicolumn{3}{|c|}{$\begin{array}{l}\text { Identified Use Category: } \\
\square \text { Mandatory Use } \\
\square \text { Continuous Use } \\
\text { Reference Use } \\
\square \text { Information Use }\end{array}$} \\
\hline \multicolumn{4}{|c|}{ Are One-Time Modifications Allowed? $\quad$ Yes $\square$ No } \\
\hline Person Signing & \multicolumn{2}{|c|}{ Signature } & Date \\
\hline \multicolumn{4}{|l|}{$\begin{array}{l}\text { Technical review: } \\
\text { James L. Huckaby }\end{array}$} \\
\hline \multicolumn{4}{|l|}{$\begin{array}{l}\text { Project Manager: } \\
\text { John Glissmeyer }\end{array}$} \\
\hline \multicolumn{4}{|l|}{$\begin{array}{l}\text { Line Manager: } \\
\text { James Droppo }\end{array}$} \\
\hline \multicolumn{4}{|l|}{ Concurrence: } \\
\hline $\begin{array}{l}\text { Quality Engineer: } \\
\text { Thomas G. Walker }\end{array}$ & & & \\
\hline
\end{tabular}




\begin{tabular}{|l|ll}
\hline PNNL Operating Procedure & $\begin{array}{l}\text { Rev. No. } 1 \\
\text { Org. Code: D9T99 }\end{array}$ & $\begin{array}{l}\text { Page } 2 \text { of 16 } \\
\text { Procedure No.: EMS-JAG-01 }\end{array}$ \\
\hline
\end{tabular}

Title: Test to Determine Uniformity of a Tracer Gas at a Sampler Probe

\section{$1.0 \quad$ Purpose}

The performance of new stack sampling systems must be shown to satisfy the requirements of 40 CFR 61, Subpart H, "National Emission standards for Emissions of Radionuclides Other than Radon from Department of Energy Facilities." This regulation governs portions of the design and implementation of effluent air sampling. The stack sampler performance is adequately characterized when potential contaminants in the effluent are of a uniform concentration at the sampling location (plane), and line losses are within acceptable limits. This procedure determines whether the concentration of gaseous contaminants is uniformly distributed in the area of the sampling probe. Other procedures address flow angle, uniformity of gas velocity, and uniformity of particulate contaminants. A contaminant concentration that is uniform at the sampling plane enables the extraction of samples that represent the true emission concentration.

The uniformity is expressed as the variability of the measurements about the mean. This is expressed using the relative coefficient of variance (COV), which is the standard deviation divided by the mean and expressed as a percentage. The lower the COV value, the more uniform the gas concentration. The acceptance criterion is that the COV of the measured gas concentrations be \# $20 \%$ across the center twothirds of the area of the stack. Furthermore, the average concentration measured at any point cannot differ from the mean of all points by more than $30 \%$.

\subsection{Applicability}

This procedure can be used in the field or on modeled stacks to determine whether air-sampling probes can collect representative samples under normal operations. The tests are applicable to effluent stacks or ducts within the following constraints:

- The tracer gas tests are generally limited to stacks with flowrates greater than 50 cubic feet per minute range. The upper bound of flowrate is determined by the sensitivity of the gas analyzer, the background reading for the tracer gas, and the availability of the tracer.

- Environmental constraints - the gas analyzer will require the use of a controlled temperature environment to maintain the equipment above 55 degrees Fahrenheit.

\subsection{Prerequisites and Conditions}

Conditions and concerns that must be satisfied before sampling are listed below:

- Safety glasses and hard toed or substantial shoes are required in the work areas.

- Properly constructed and inspected work platforms may be needed to access the test ports.

- Scaffold-user or fall protection training may be required in some instances to access the sampling ports of the stack.

- Alcohol may be used for equipment cleanup. A flammable equipment storage cabinet is required to flammable chemicals. 


\begin{tabular}{|l|lc|}
\hline PNNL Operating Procedure & $\begin{array}{l}\text { Rev. No. 1 } \\
\text { Org. Code: D9T99 }\end{array}$ & $\begin{array}{l}\text { Page 3 of } 16 \\
\text { Procedure No.: EMS-JAG-01 }\end{array}$ \\
\hline \multicolumn{2}{|c|}{ Title: Test to Determine Uniformity of a Tracer Gas at a Sampler Probe } \\
\hline
\end{tabular}

- Familiarity with the use and operation of gas delivery systems and the ability to detect concentration build-ups of the gas is essential to avoid exceeding ACGIH concentration for the tracer gas.

- Knowledge of the setup, use of, and operation of flowmeters, gas analyzers, and computers is essential.

- A job-hazards analysis may be required in certain cases.

\subsection{Precautions and Limitations}

Caution: The American Conference of Governmental Industrial Hygienists (ACGIH) 8-hour timeweighted average limit for human exposure to sulfur hexafluoride gas is $1000 \mathrm{ppm}\left(6,000 \mathrm{mg} / \mathrm{m}^{3}\right)$. It is colorless and odorless.

During tests of stacks with high flow rates, sulfur hexafluoride will be injected at a high rate into the base of the stack to overcome the large dilution factor needed to detect the tracer at the sampling ports above. If a leak occurs in the gas delivery system, the potential is present for a buildup of $\mathrm{SF}_{6}$ to occur that could approach the 1000-ppm level. The gas is five times as heavy as air, so it will accumulate in confined spaces and in low areas. Leak tests of the delivery system will be made at least daily to prevent such an occurrence.

Access to the test ports may require the use of scaffolding or manlifts, either of which will necessitate special training for sampling personnel and any observers. The training requirements will be indicated in the job hazard analysis. This will limit access to the sampling ports to trained personnel.

If the purpose of a given run is to investigate the sensitivity of the COV determination to the tracerinjection location, the test may be invalid if the ending ambient concentration is elevated above that at the start of the test. This would indicate poor dispersion away from the test site and recirculation of the tracer to the inlet of the fan if the stack exhaust point is in view of and is reasonably close to the fan inlet. This may result in a false indication of good mixing.

\subsection{Equipment Used for Stack Measurements}

Specific calibration check concentration levels, probe dimensions, measurement grids, flow rates, and other special requirements will be provided in the specific Test Instruction. Exhibit A provides a typical layout for the test setup. The following are essential items of equipment:

- Sulfur hexafluoride calibration check gas

- Sulfur hexafluoride bulk gas 


\begin{tabular}{|l|ll|}
\hline PNNL Operating Procedure & $\begin{array}{l}\text { Rev. No. 1 } \\
\text { Org. Code: D9T99 }\end{array}$ & $\begin{array}{l}\text { Page 4 of 16 } \\
\text { Procedure No.: EMS-JAG-01 }\end{array}$ \\
\hline Title: Test to Determine Uniformity of a Tracer Gas at a Sampler Probe \\
\hline
\end{tabular}

- Bruel and Kjaer Model 1302 Gas analyzer

- Gas regulators and flowmeters

- Gas sampling probe

- Gas injection probe

- Vacuum pump (Sierra)

- Air velocity meter

The absolute calibration of the Model 1302 Gas Analyzer is not as important as its general response because the concentration data are used in a relative manner in calculating the COV and in plotting the concentrations at the measurement points. Consequently, the analyzer is Category 2 MTE (user calibrated) and will be checked against a calibrated gas mixture before and after the series of tests, and the instrument's response may be checked on a daily basis. Agreement within $10 \%$ of the calibration gas is acceptable.

\subsection{Work Instructions for Setup, Measurements, and Data Reduction}

The steps taken to setup, configure, and operate the stack fans and test equipment are listed. Based on previous field measurements, the steps are ordered to achieve maximum efficiency in the testing. In addition to these steps, test instructions, which are developed for each test series, provide specific details and operating parameters.

\subsection{Preliminary Steps:}

6.1.1 Provide essential supplies at the sampling location. (gas cylinders and regulators, fittings and probe-port couplers, marking pens, data sheets, writing, and probe-supporting platforms).

6.1.2 Fill in test information on data form.

6.1.3 Obtain barometric, temperature, and relative-humidity information for the gas analyzer.

6.1.4 Set up the gas analyzer system at the stack sampling port according to the illustrations in Exhibits A and B. 


\begin{tabular}{|l|ll|}
\hline PNNL Operating Procedure & $\begin{array}{l}\text { Rev. No. 1 } \\
\text { Org. Code: D9T99 }\end{array}$ & $\begin{array}{l}\text { Page } 5 \text { of 16 } \\
\text { Procedure No.: EMS-JAG-01 }\end{array}$ \\
\hline
\end{tabular}

Title: Test to Determine Uniformity of a Tracer Gas at a Sampler Probe

Note: The sampling equipment consists of a stainless steel probe with enough length to reach across the inside diameter of the stack, allowing for fittings. The intake end should have a $90^{\circ}$ bend so that the open end of the tube faces downward or into the flow within the stack). The outlet end of the probe should terminate in a tee. One leg of the tee connects by flexible tubing to a rotameter and vacuum pump. This leg should draw from 1- to 10-lpm flow of air, depending on the volumetric flow in the stack. The other leg of the tee connects via flexible tubing to a coarse inline filter (47-mm-diameter glass fiber filter) and then to the Model 1302 gas analyzer inlet. To minimize tubing length to the analyzer, locate the gas analyzer near the test port on the stack.

\subsection{System Startup}

6.2.1 If not already running, start the stack fan, adjust the flow to the velocity called for in the test instruction, and record on the data sheet.

6.2.2 Verify the stack centerline air velocity in the sampling plane using a velocity flow meter, and record value on data sheet.

\subsubsection{Turn-on the gas analyzer.}

6.2.4 Program the analyzer for:

- 60-second samples,

- continuous operation,

- the current barometric pressure,

- moisture compensation if needed.

Note: Gas analyzer readings can be made with or without water-vapor correction. If the air is sufficiently dry ( $<$ than about $60 \%$ relative humidity) where the water vapor contribution is negligible ( $<$ than about $14.5 \mathrm{E}+03 \mathrm{ppm}$ ), the balance of the readings can be made with water vapor compensation but without water vapor measurement to reduce sample times.

6.2.5 Set the sample probe to the center position.

Note: Mark the sampling probe with a permanent marker so the inlet can be placed at each successive measurement point. The layout for the sample points is given in the test instruction. 


\begin{tabular}{l|ll}
\hline PNNL Operating Procedure & $\begin{array}{l}\text { Rev. No. 1 } \\
\text { Org. Code: D9T99 }\end{array}$ & $\begin{array}{l}\text { Page 6 of 16 } \\
\text { Procedure No.: EMS-JAG-01 }\end{array}$ \\
\hline
\end{tabular}

Title: Test to Determine Uniformity of a Tracer Gas at a Sampler Probe

Note: Sampling plane traverse points Use the grid of measurement points provided with the tests instruction and dataform. This is usually the same as used for the velocity uniformity test. A centerpoint, is included as a common reference and for graphical purposes. The layout design divides the area of the sampling plane so that each point represents approximately an equal-sized area.

\subsection{Daily Tracer Gas Background Concentration Measurement}

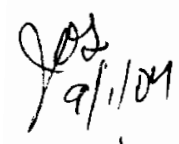

6.3.1 At the beginning of sampling each day and after the analyzer has stabilized (about 10 minutes), obtain at least six consecutive background readings. Do not proceed with the test if the background exceeds $5 \%$ of the anticipated average concentration in the stack.

6.3.2 Record these readings in the logbook designated for the tests.

\subsection{Gas Injection and Sample Collection}

The injection equipment consists of a pressurized cylinder of pure liquid sulfur hexafluoride that converts to gas when released. The setup is shown in the figure in Exhibit B and includes a gas regulator, valve, flowmeter (rotameter), flexible tubing, and a stainless steel injection probe with a $90 \mathrm{E}$ bend at the discharge end, which is secured at one of five positions. The connections and fittings should be checked to ensure that they are secure and leak free to prevent the loss of gas.

\section{Note: Location of Tracer Gas Injection Points}

Injection plane - The tests are repeated using five tracer gas injection points (at the centerpoint and at four orthogonally spaced points) within the injection plane.

These four points are located near the corners if the duct cross section is rectangular. The distance from these four points to the corner or wall is less than $25 \%$ of the duct's hydraulic diameter (HD), which is calculated by $\quad H D=\frac{2 H W}{H+W}$

where $\mathrm{H}$ and $\mathrm{W}$ are the height and width of a rectangular duct $(\mathrm{H}$ and $\mathrm{W}$ are the same in a round duct). More specific dimensions are given in the Test Instruction. 
Rev. No. 1

Org. Code: D9T99
Page 7 of 16

Procedure No.: EMS-JAG-01

Title: Test to Determine Uniformity of a Tracer Gas at a Sampler Probe

6.4.1 Position the injection probe, according to the test instruction

found as Attachment A.

6.4.2 Start injection of the tracer gas and adjust for flow rate specified in the test instruction and note the time.

\section{Note: Estimation of Sulfur Hexafluoride Injection Rate}

Estimate the $\mathrm{SF}_{6}$ injection rate so the average diluted concentration will be within the range of 10 to $100 \%$ of the concentration of the calibration check gas according to the following equation:

$$
\text { injection flowrate }=\text { stack flowrate } \times \frac{\text { target }}{10^{6}}
$$

The rotameter reading should be adjusted for the density of the $\mathrm{SF}_{6}$. The air equivalent reading is

$$
\text { rotameter reading }=k x \text { actual flowrate }
$$

where $k$ is 2.53 (the square-root of the density) for $\mathrm{SF}_{6}$.

6.4.3 On the data sheet, label the columns of data according to the directions of the traverses.

6.4.4 Verify that the directional orientations and the numbered sample positions are consistent.

6.4.5 Position the sample probe at each measurement point in succession, and record the reading on the dataform.

Note: Each test relies on one repetition for each measurement point in each traverse direction, repeated three times. The repeats are made as three separate runs and not as three consecutive measurements at each point. 


\begin{tabular}{|l|ll}
\hline PNNL Operating Procedure & $\begin{array}{l}\text { Rev. No. 1 } \\
\text { Org. Code: D9T99 }\end{array}$ & $\begin{array}{l}\text { Page } 8 \text { of 16 } \\
\text { Procedure No.: EMS-JAG-01 }\end{array}$ \\
\hline
\end{tabular}

Title: Test to Determine Uniformity of a Tracer Gas at a Sampler Probe

6.4.6 Perform two additional repetitions of Step 6.4.5. above

6.4.7 Switch the tests to the other direction and repeat Steps 6.4.5

and 6.4.6.

6.4.8 Check the data sheet for completeness.

6.4.9 Record the final

- Rotameter flow rate

- Time since the start of gas injection

- Pressure in the gas cylinder.

6.4.10 Shut down the delivery of tracer gas.

6.4.11 Continue operation of the gas analyzer for several minutes to purge any remaining gas through the analyzer.

6.4.12 Measure the background tracer gas concentration and record the levels on the data sheet.

6.4.13 Record any climatic conditions that have changed on the data sheet.

6.4.14 Enter the centerline stack velocity flow on the data sheet.

6.4.15 Record any deviations from the above procedure on the data sheet.

6.4.16 Repeat steps 6.4.1 - 6.4.15 for each run as indicated in the Test Instruction.

\subsection{Data Recording and Calculations}

Prepare the electronic data sheet on which to enter gas concentration readings and other information relevant to the test (see test instruction). 


\begin{tabular}{l|ll}
\hline PNNL Operating Procedure & $\begin{array}{l}\text { Rev. No. } 1 \\
\text { Org. Code: D9T99 }\end{array}$ & $\begin{array}{l}\text { Page } 9 \text { of } 16 \\
\text { Procedure No.: EMS-JAG-01 }\end{array}$ \\
\hline
\end{tabular}

Title: Test to Determine Uniformity of a Tracer Gas at a Sampler Probe

6.5.1 Review the raw data sheets for completeness.

6.5.2 Enter the data into the electronic data sheet.

6.5.3 Calculate the COV for the run.

Note: The EXCEL datasheet shown in Appendix C is set up to calculate the COV for each tracer gas concentration traverse using the average concentration data from all points in the inner two-thirds of the cross section area of the plane (including the center point).

6.5.4 Compare the observed COV for each run to the acceptance criterion.

Note: The test is acceptable if the COV is within $\# 20 \%$ for the inner two-thirds of the stack diameter and if no point differs from the mean by more than $30 \%$. This is determined by inspecting the average concentration at each measurement point. The COV is 100 times the standard deviation divided by the mean.

6.5.5 Sign and date the data sheet attesting to its validity.

Note: A separate datasheet will be provided and signed-off for each test run.

\subsection{Gas Analyzer Calibration Check Steps}

Check the gas analyzer calibration by subjecting the analyzer to sulfur hexafluoride calibration gas. Refer to the analyzer's manual, parts 2 and 4. 
6.6.1 Set up the system for gas analysis with the regulator, the valve, flexible tubing, and a tee with one leg exhausting excess gas through a flowmeter and the other leg attached to the inlet of the Model 1302. Program the units of measurement as in Part 4.2.3. Enter the barometric pressure in $\mathrm{mm} \mathrm{Hg}$ pressure, standard temperature (that used by the calibration gas vendor), and the sampling tube length into the environmental setup (Part 4.2.4). Record the information on the data sheet.

6.6.2 Set the Model 1302's clock. Program the analyzer for water compensation, but not water measurement, at 1 -minute continuous measurement mode (according to Part 4.4.2 in manual).

6.6.3 Program for a continuous monitoring task (4.2.5), and initiate monitoring (4.2.6).

6.6.4 Monitor room conditions, and record the data for several measurements by sampling zero air or room air for at least 5 minutes.

Note: If the test location has a buildup of the gas, a zero air cylinder or clean air supply will be needed. The $\mathrm{SF}_{6}$ concentration in the room should be several orders of magnitude below the calibration-gas. These settings optimize the low detection capabilities of the acoustically-based detection system.

6.6.5 Sample calibration gases (from lowest available concentrations to highest) for at least five readings each or until no observable trend is found. Record the identification of the calibration gas used. Record data and results in the Logbook.

Note: Set the calibration gas flow rate high enough to ensure that the glass ball in the rotameter does not drop to zero during any of the observed steps of a sample cycle. As the calibration check continues, gas levels exhausted during the check will be released into the room, and the $\mathrm{SF}_{6}$ background concentrations will increase as the analyzer is checked. The $\mathrm{SF}_{6}$ reading should be within $10 \%$ of the calibration-gas concentration, and the water content should be much lower than ambient. 


\begin{tabular}{|l|lc|}
\hline PNNL Operating Procedure & $\begin{array}{l}\text { Rev. No. 1 } \\
\text { Org. Code: D9T99 }\end{array}$ & $\begin{array}{l}\text { Page 11 of 16 } \\
\text { Procedure No.: EMS-JAG-01 }\end{array}$ \\
\hline
\end{tabular}

Woy 6.6.6 Obtain baseline tracer (calibration gas) readings at the end $9 / 1 / 01$ of the calibration check. Record results on the data sheet.

Motinted on sheet. all roodeng

Note: The reading will generally be recorded from the digital concentration display. It may be convenient to record the data on a printer or computer, which can be coupled to the analyzer. See the Manual Part 12 (especially Part 12.2.5) for connecting to a printer in data $\log$ mode. were 


\begin{tabular}{|l|ll}
\hline PNNL Operating Procedure & $\begin{array}{l}\text { Rev. No. 1 } \\
\text { Org. Code: D9T99 }\end{array}$ & $\begin{array}{l}\text { Page } 12 \text { of } 16 \\
\text { Procedure No.: EMS-JAG-01 }\end{array}$ \\
\hline
\end{tabular}

Title: Test to Determine Uniformity of a Tracer Gas at a Sampler Probe

\subsection{Exhibits/Attachments}

Exhibit A - Overview of Stack and Injection/Sampling Setups

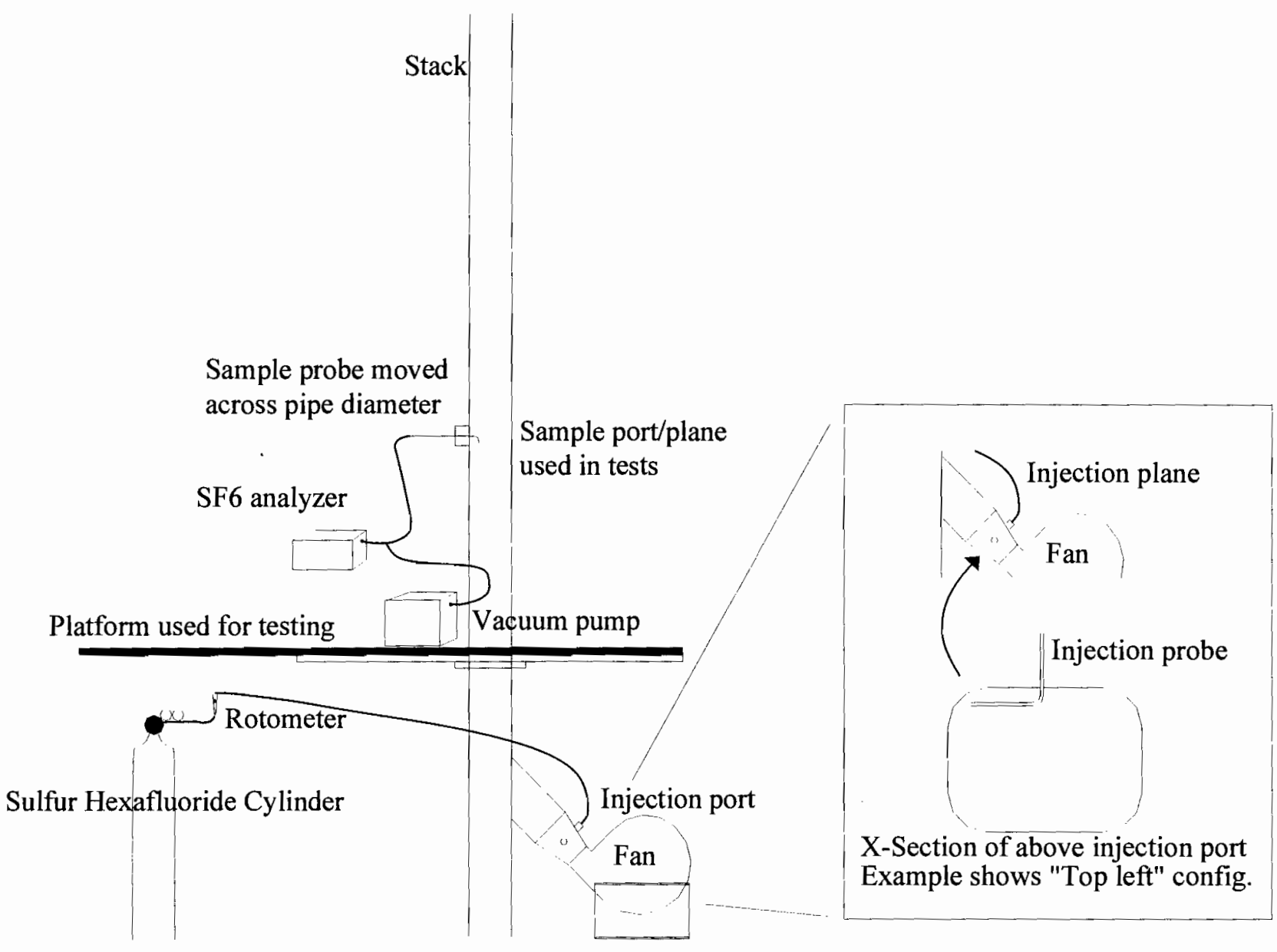




\begin{tabular}{|r|ll|}
\hline FNN Operating Procedure & $\begin{array}{l}\text { Rev. No. } 1 \\
\text { Org. Code: D9T99 }\end{array}$ & $\begin{array}{l}\text { Page 13 of 16 } \\
\text { Procedure No.: EMS-JAG-01 }\end{array}$ \\
\hline \multicolumn{2}{|c|}{ Title: Test to Determine Uniformity of a Tracer Gas at a Sampler Probe } \\
\hline
\end{tabular}

Exhibit B - Details for Stack Sampling Probe and Gas Analyzer Setup

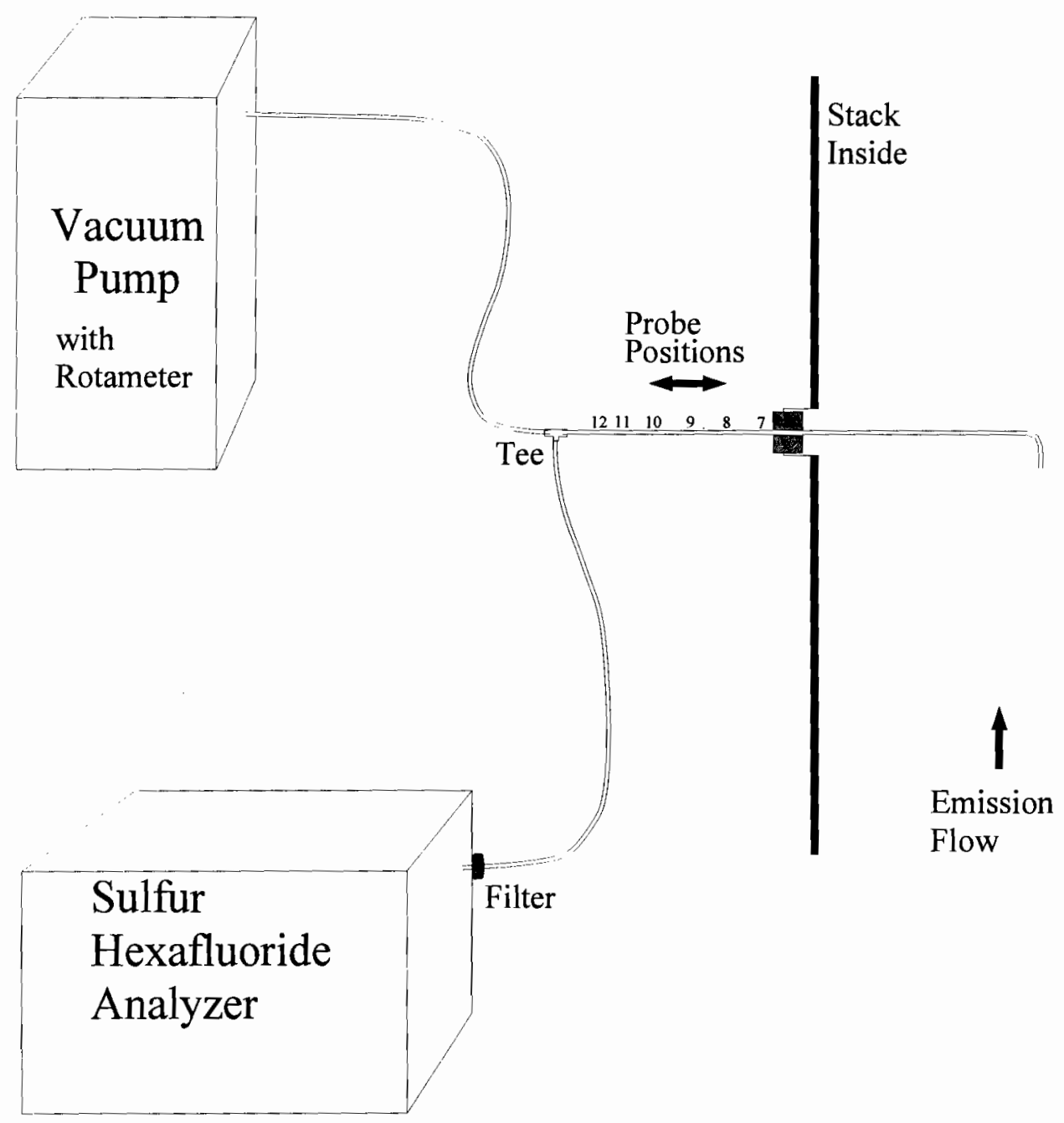




\begin{tabular}{l|ll}
\hline PNNL Operating Procedure & $\begin{array}{l}\text { Rev. No. 1 } \\
\text { Org. Code: D9T99 }\end{array}$ & $\begin{array}{l}\text { Page 14 of 16 } \\
\text { Procedure No.: EMS-JAG-01 }\end{array}$
\end{tabular}

\section{Title: Test to Determine Uniformity of a Tracer Gas at a Sampler Probe}

Exhibit C - Example EXCEL Data Sheet

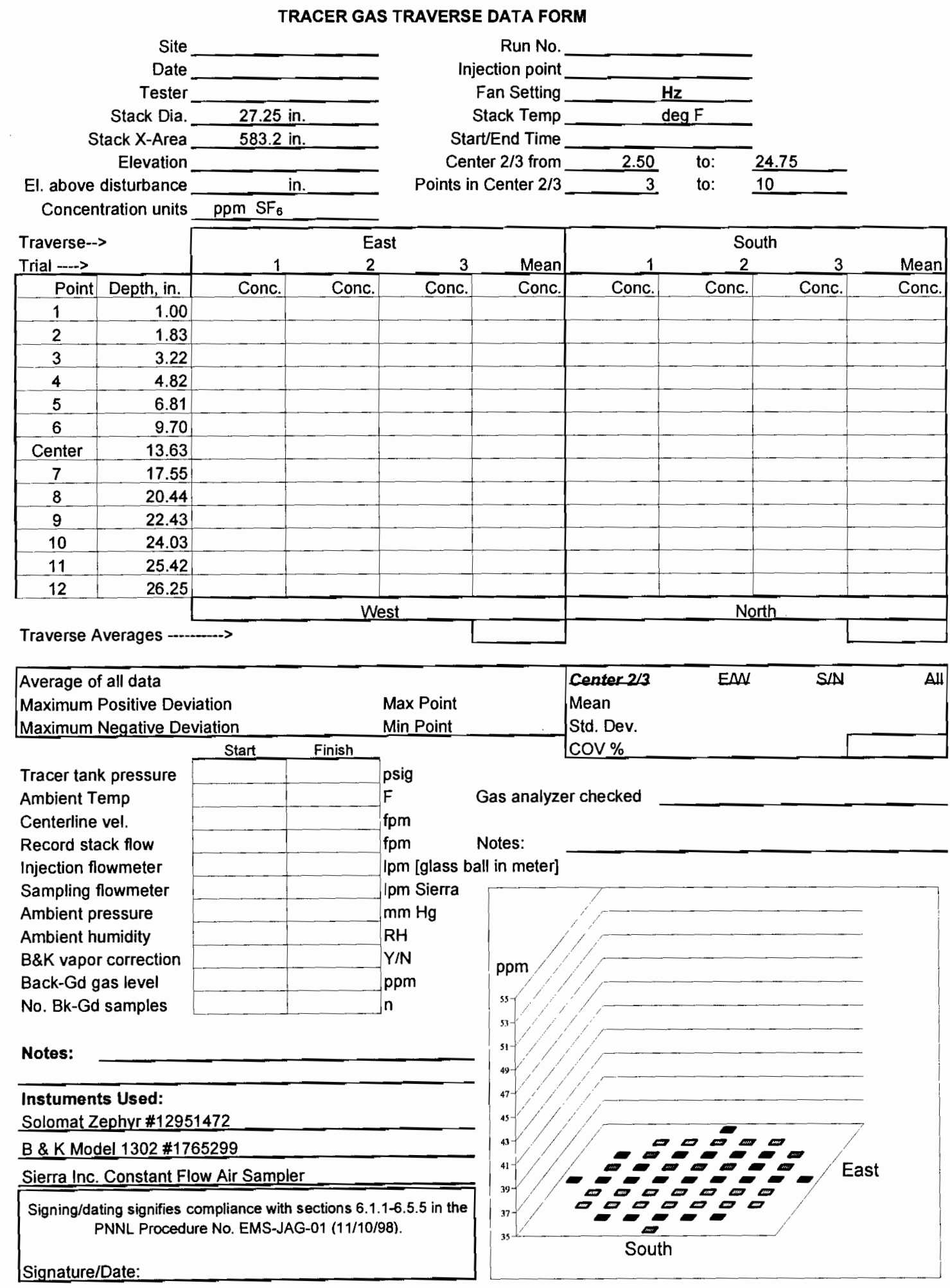




\begin{tabular}{|l|ll|}
\hline PNNL Operating Procedure & $\begin{array}{l}\text { Rev. No. 1 } \\
\text { Org. Code: D9T99 }\end{array}$ & $\begin{array}{l}\text { Page 15 of 16 } \\
\text { Procedure No.: EMS-JAG-01 }\end{array}$ \\
\hline
\end{tabular}

Attachment A - Illustrative Test Instruction. 
Title: Test to Determine Uniformity of a Tracer Gas at a Sampler Probe

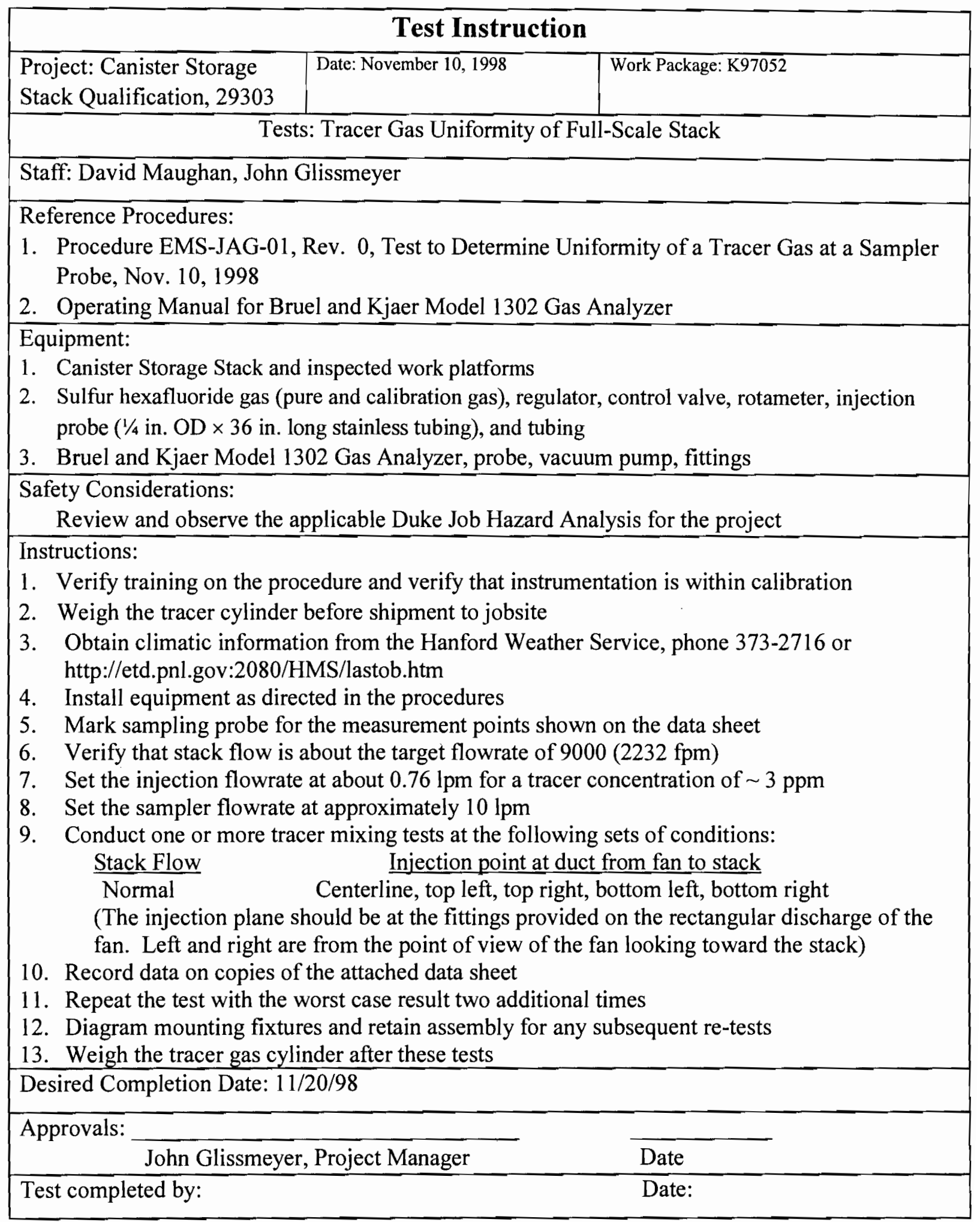


INDEPENDENT TECHNICAL REVIEW RECORD

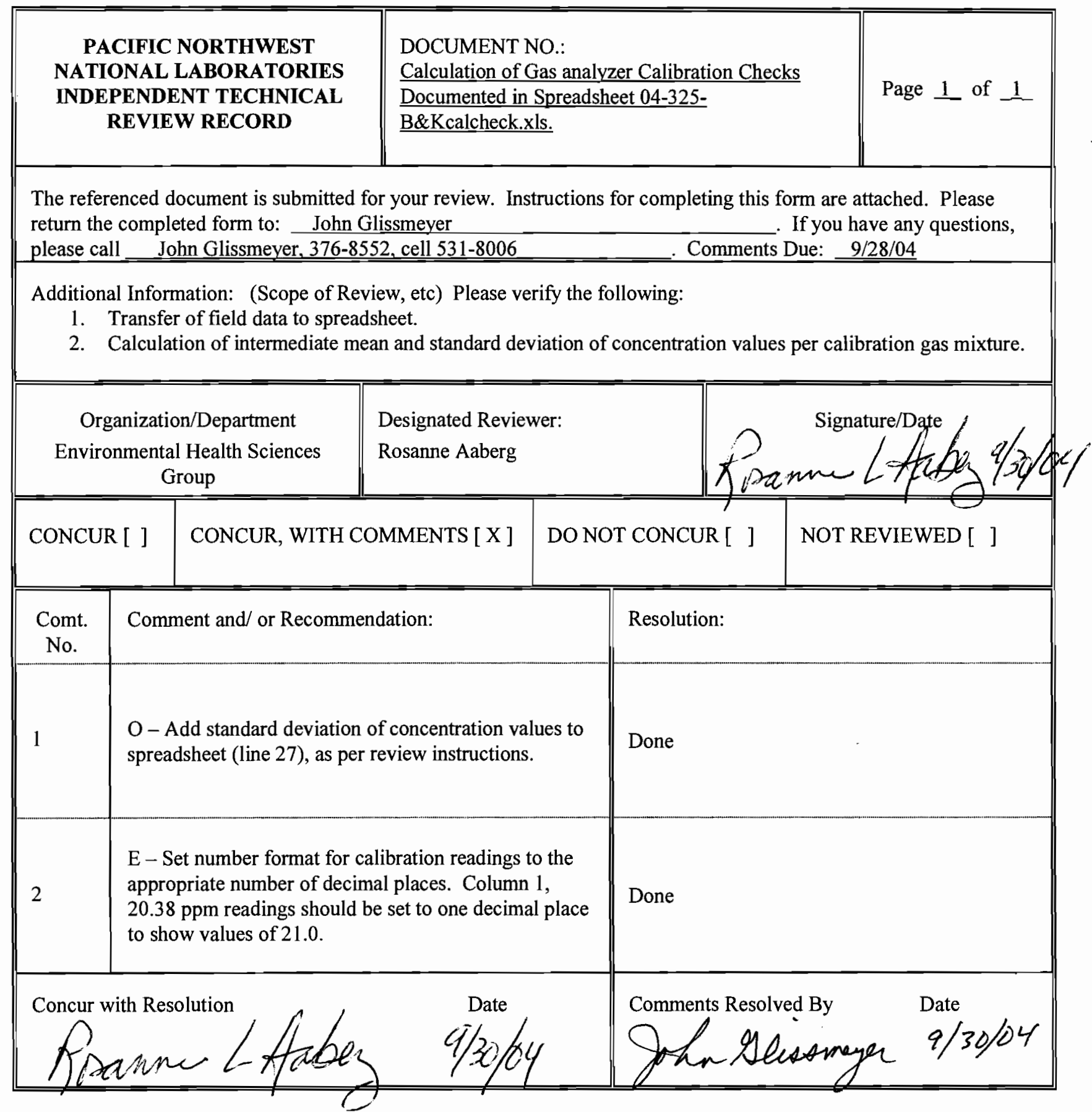




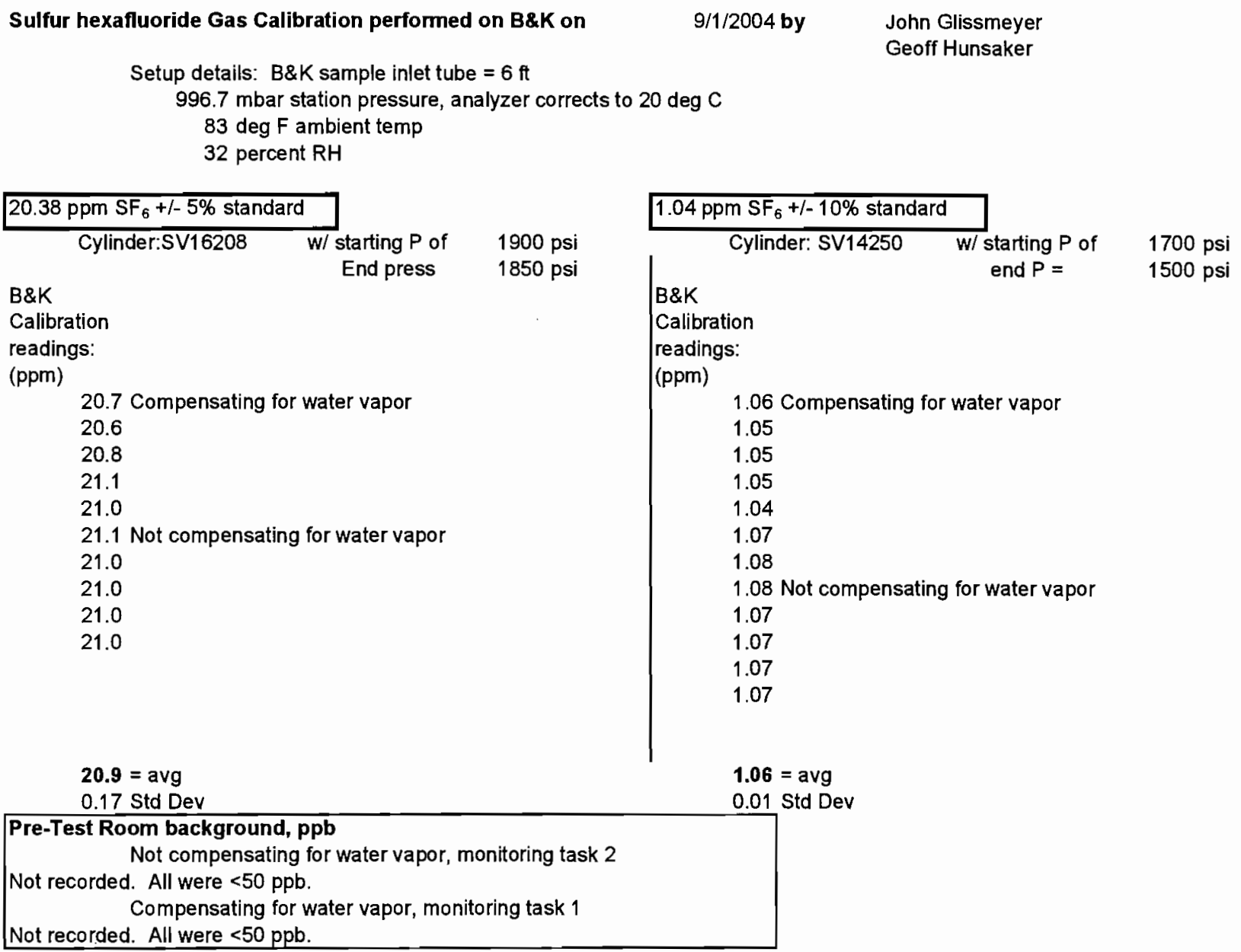

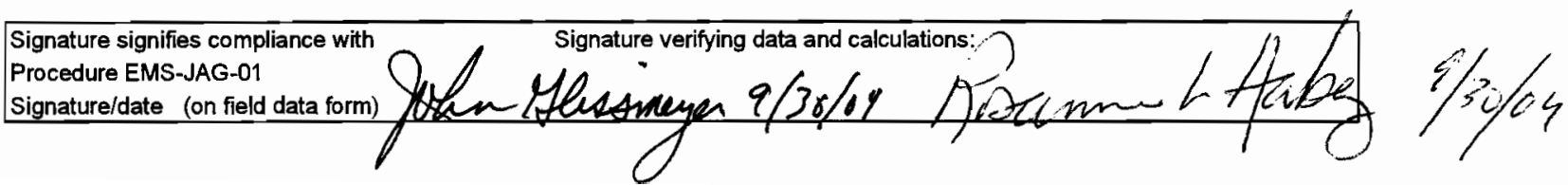





\section{Appendix F}

\section{Particle Tracer Gas Uniformity Procedure and Data}





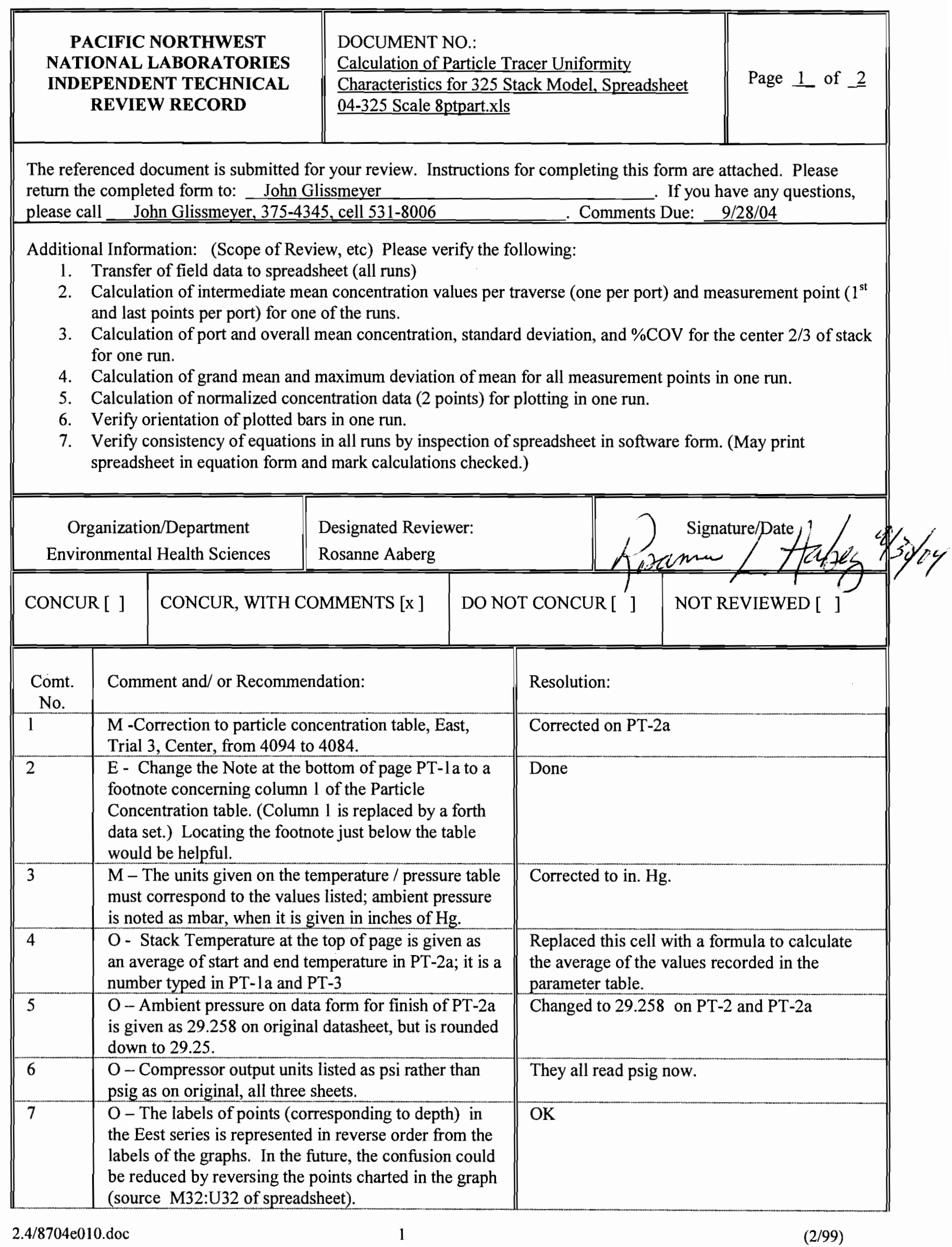




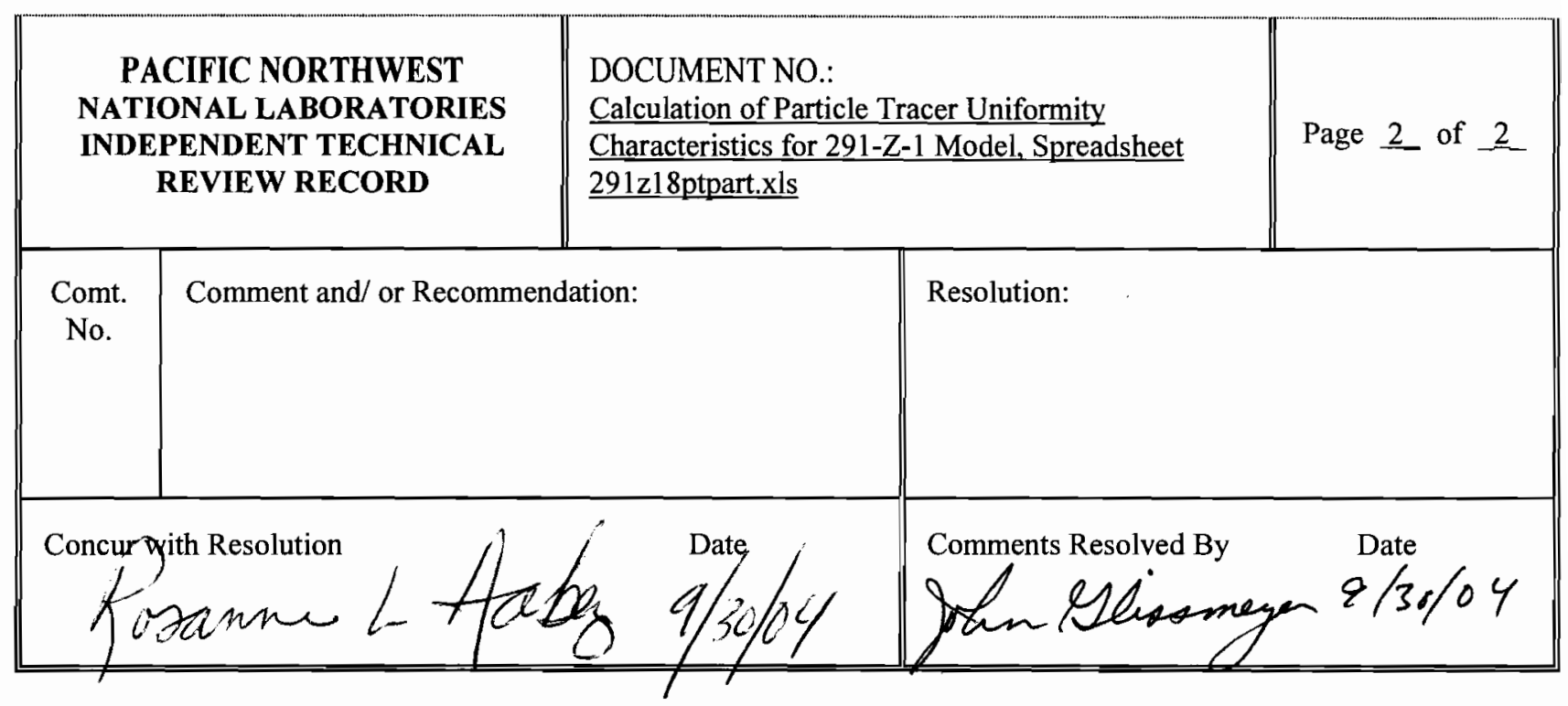

2.4/8704e010.doc

2

(2/99) 


\section{PARTICLE TRACER TRAVERSE DATA FORM}

Site 325 Model Stack

Date 9/16/2004

Tester JAG/MYB/JMB

Stack Dia.

Stack X-Area

Elevation N.A.

Distance to disturbance

Measurement units particles/ft3

Order -.--->

Traverse-->

Trial $\ldots-.>$
18 in.

254.5 in. 2

120 inches 2nd

Run No. PT-1

Fan configuration Far Fan

Fan Setting $37.1 \overline{\mathrm{Hz}}$

Stack Temp

$80 \operatorname{deg} F$

Start/End Time 2:18 PM/ 4:30 PM

Center $2 / 3$ from

Points in Center $2 / 3$ 1.65 to

Injection Point Dwnstrm of far fan, centerline

\begin{tabular}{|c|c|c|c|c|c|c|c|c|c|}
\hline \multirow{3}{*}{\multicolumn{2}{|c|}{$\begin{array}{l}\text { Order -----> } \\
\text { Traverse--> }\end{array}$}} & \multicolumn{4}{|c|}{ 2nd } & \multicolumn{4}{|c|}{$1 \mathrm{st}$} \\
\hline & & \multicolumn{4}{|c|}{ East } & \multicolumn{4}{|c|}{ North } \\
\hline & & 1 & 2 & 3 & Mean & 1 & 2 & 3 & Mean \\
\hline Point & Depth, in. & \multicolumn{4}{|c|}{ particles/ft3 } & \multicolumn{4}{|c|}{ particles/ft3 } \\
\hline 1 & 0.58 & 2579 & 2506 & 2538 & 2541.0 & 2524 & 2067 & 2059 & 2216.7 \\
\hline 2 & 1.89 & 2694 & 2438 & 2278 & 2470.0 & 2440 & 1953 & 1799 & 2064.0 \\
\hline 3 & 3.49 & 2616 & 2443 & 2133 & 2397.3 & 2402 & 2109 & 1931 & 2147.3 \\
\hline 4 & 5.81 & 2528 & 2539 & 2309 & 2458.7 & 2068 & 1904 & 1923 & 1965.0 \\
\hline Center & 9.00 & 2403 & 2568 & 2596 & 2522.3 & 2855 & 1928 & 2014 & 2265.7 \\
\hline 5 & 12.19 & 2272 & 2550 & 2427 & 2416.3 & 3089 & 2513 & 2022 & 2541.3 \\
\hline 6 & 14.51 & 2473 & 2421 & 2692 & 2528.7 & 3024 & 2620 & 2063 & 2569.0 \\
\hline 7 & 16.11 & 2265 & 2274 & 2298 & 2279.0 & 4035 & 2782 & 2126 & 2981.0 \\
\hline 8 & 17.42 & 2409 & 2464 & 2010 & 2294.3 & 4183 & 3298 & 3443 & 3641.3 \\
\hline Averages & & 2471.0 & 2467.0 & 2364.6 & 2434.2 & 2957.8 & 2352.7 & 2153.3 & 2487.9 \\
\hline
\end{tabular}
1st

\begin{tabular}{|c|c|c|c|c|c|c|c|}
\hline $4 / I$ & $\mathrm{pt} / \mathrm{ft} 3$ & Dev. from mean & Center 2/3 & East & North & All & Normlzd \\
\hline ean & 2461.1 & & Mean & 2438.9 & 2361.9 & $2400 . \overline{40}$ & 2534.19 \\
\hline in Point & 1965.0 & $-20.2 \%$ & Std. Dev. & 85.8 & 355.9 & 251.90 & 292.62 \\
\hline Max Point & 3641.3 & $48.0 \%$ & cov as $\%$ & 3.5 & 15.1 & 10.49 & 11.55 \\
\hline
\end{tabular}

\section{Avg Conc $2469 \mathrm{pt} / \mathrm{ft} 3$}

Generator Inlet Press

Stack Temp

Centerline vel.

Ambient pressure

Ambient humidity

Ambient temp

Back-Gd aerosol

No. Bk-Gd samples

Compressor output reg

Optical Particle Counters:

\begin{tabular}{lll}
\hline Met One A2408 & S/N 96258675 & Cal 8/5/04 \\
\hline
\end{tabular}

Pre-test stability readings: $2248,2160,2200,2210$,

2091, 1980, 1908, 2033, 2005

Oil Used: FisherBrand 19

Probe has a 4.5-in throw so is that much closer to the

stack than the port.

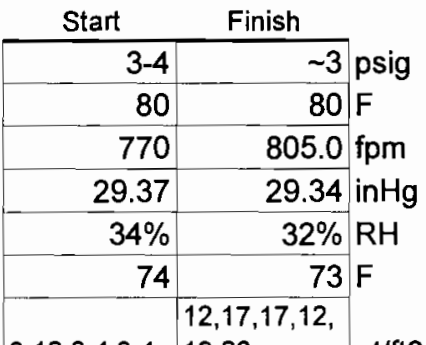

$3,12,3,4,3,4 \quad 19,20 \quad \mathrm{pt} / \mathrm{ft} 3$

6

115 psig
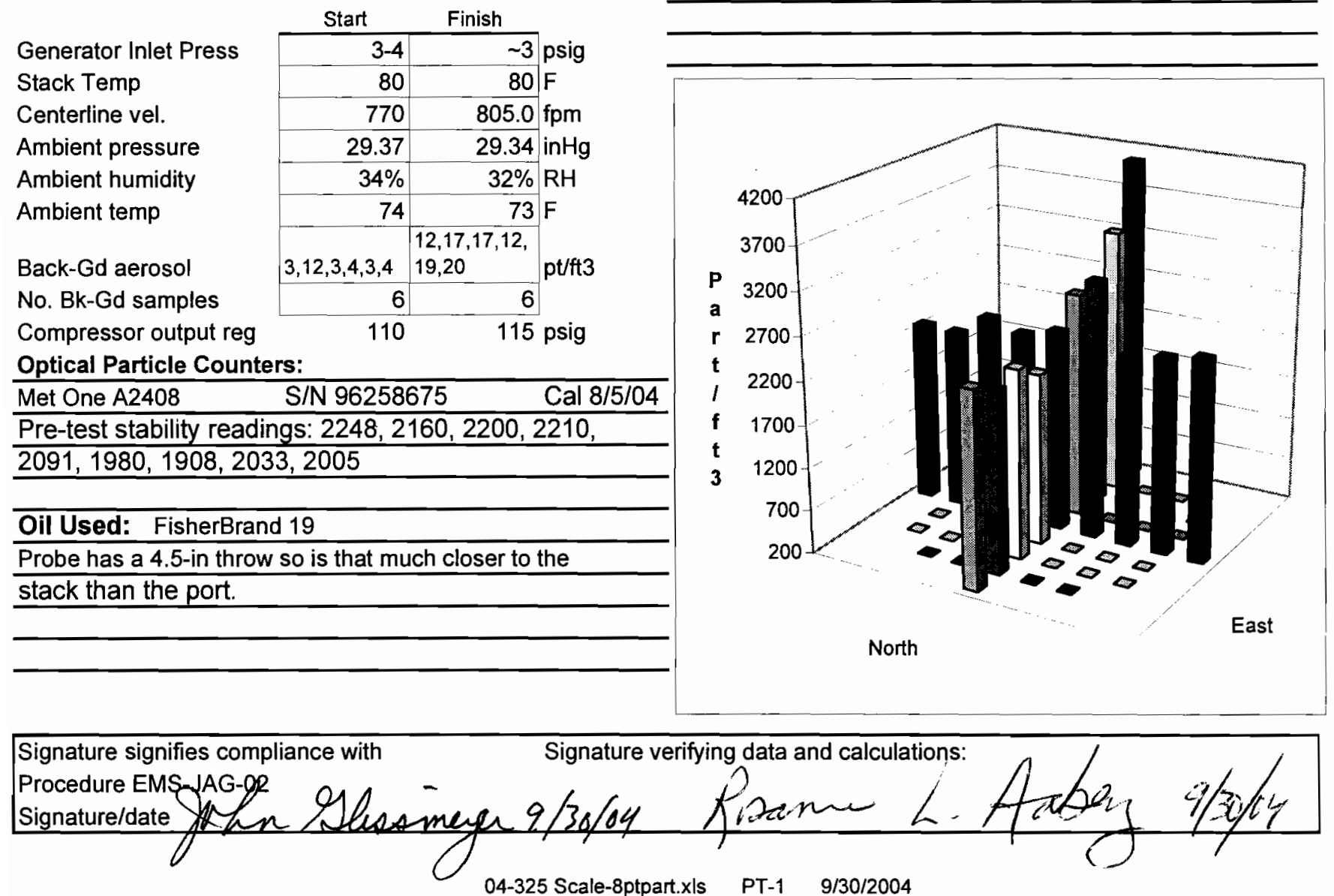
PARTICLE TRACER TRAVERSE DATA FORM

Site 325 Model Stack

Date 9/16/2004

Tester JAG/MYB/JMB

Stack Dia.

Stack X-Area 254.5 in.2

Elevation N.A.

Distance to disturbance

Measurement units particles/ft3

Order ---.->

Traverse-->

Trial $\rightarrow$
Run No. PT-1a

Fan configuration Far Fan

Fan Setting $37.1 \mathrm{~Hz}$

Stack Temp $80 \operatorname{deg} F$

Start/End Time 2:18 PM/ 4:30 PM

Center $2 / 3$ from

Points in Center $2 / 3$ Injection Point Dwnstrm of far fan, centerline

\begin{tabular}{|c|c|c|c|c|c|c|c|c|c|}
\hline Trial --->> & & 1 & 2 & 3 & Mean & 1 & 2 & 3 & Mean \\
\hline Point & Depth, in. & \multicolumn{4}{|c|}{ particles/ft3 } & \multicolumn{4}{|c|}{ particles/ft3 } \\
\hline 1 & 0.58 & 2579 & 2506 & 2538 & 2541.0 & 1834 & 2067 & 2059 & 1986.7 \\
\hline 2 & 1.89 & 2694 & 2438 & 2278 & 2470.0 & 1877 & 1953 & 1799 & 1876.3 \\
\hline 3 & 3.49 & 2616 & 2443 & 2133 & 2397.3 & 2069 & 2109 & 1931 & 2036.3 \\
\hline 4 & 5.81 & 2528 & 2539 & 2309 & 2458.7 & 2048 & 1904 & 1923 & 1958.3 \\
\hline Center & 9.00 & 2403 & 2568 & 2596 & 2522.3 & 2352 & 1928 & 2014 & 2098.0 \\
\hline 5 & 12.19 & 2272 & 2550 & 2427 & 2416.3 & $21 \overline{96}$ & 2513 & 2022 & 2243.7 \\
\hline 6 & 14.51 & 2473 & 2421 & 2692 & 2528.7 & 2184 & 2620 & 2063 & 2289.0 \\
\hline 7 & 16.11 & 2265 & 2274 & 2298 & 2279.0 & 3462 & 2782 & 2126 & 2790.0 \\
\hline 8 & 17.42 & 2409 & 2464 & 2010 & 2294.3 & 2969 & 3298 & 3443 & 3236.7 \\
\hline Averages - & $->>$ & 2471.0 & 2467.0 & 2364.6 & 2434.2 & 2332.3 & 2352.7 & 2153.3 & 2279.4 \\
\hline
\end{tabular}

NOTE: Column 1 North replaced with fourth data set which was taken after noticing a steady decline in concentrati with respective measurements starting with North 1,2 , then 3 .

Concern that more time was needed to stabilize after starting up particle generator.

\begin{tabular}{|lrr|lrrrr|r|}
\hline All & pt/ft3 & Dev. from mean & Center 2/3 & East & North & \multicolumn{2}{c|}{ All } & Normlzd \\
Mean & 2356.8 & & Mean & 2438.9 & 2184.5 & 2311.71 & $\frac{2532.63}{253}$ \\
Min Point & 1876.3 & $-20.4 \%$ & Std. Dev. & 85.8 & 304.6 & 252.26 & 273.40 \\
Max Point & 3236.7 & $37.3 \%$ & CoV as \% & 3.5 & 13.9 & $\mathbf{1 0 . 9 1}$ & $\mathbf{1 0 . 7 9}$ \\
\hline
\end{tabular}

Avg Conc $2363 \mathrm{pt} / \mathrm{ft} 3$

Generator Inlet Press

Stack Temp

Centerline vel.

Ambient pressure

Ambient humidity

Ambient temp

Back-Gd aerosol

No. Bk-Gd samples

Compressor output reg

Optical Particle Counters:

Met One A2408

S/N 96258675

Pre-test stability readings: $2248,2160,2200,2210$

2091, 1980, 1908, 2033, 2005

Oil Used: FisherBrand 19

Probe has a 4.5-in throw so is that much closer to the stack than the port.

\begin{tabular}{|c|c|c|}
\hline Start & Finish & \\
\hline 3-4 & $\sim 3$ & psig \\
\hline 80 & 80 & $F$ \\
\hline 770 & 805.0 & $\mathrm{fpm}$ \\
\hline 29.37 & 29.34 & inHg \\
\hline $34 \%$ & $32 \%$ & $\mathrm{RH}$ \\
\hline 74 & $\begin{array}{r}73 \\
\end{array}$ & $F$ \\
\hline $3,12,3,4,3,4$ & $\begin{array}{l}12,17,17,12 \\
19,20\end{array}$ & \\
\hline 6 & 6 & \\
\hline 110 & 115 & psi \\
\hline
\end{tabular}

\section{Instuments Used:}

TSI Velocity Calc Plus S/N 209060

Calib 8/25/04

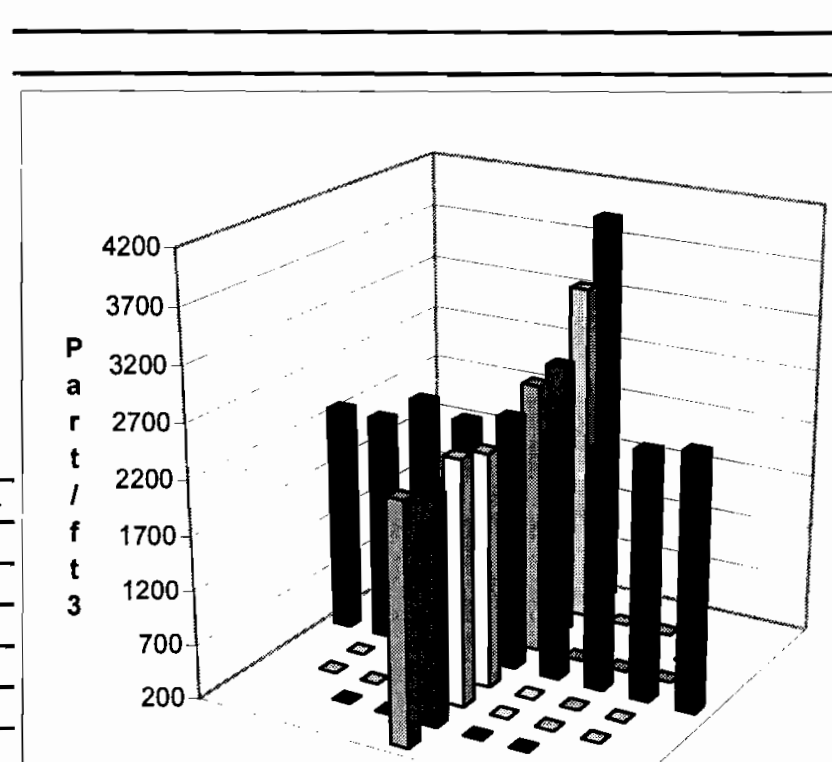

North

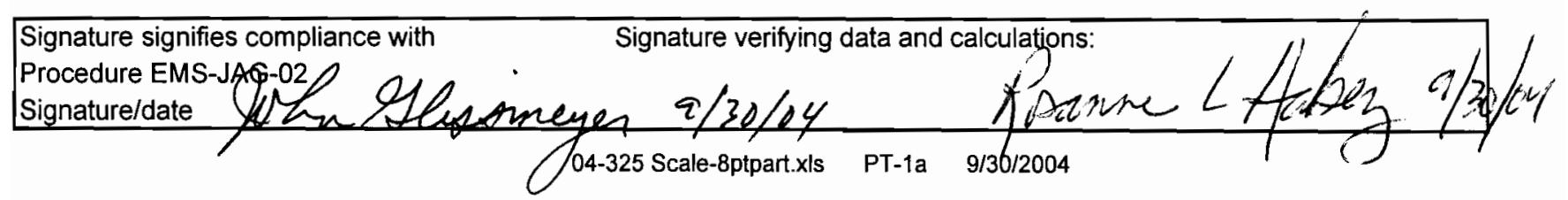


PARTICLE TRACER TRAVERSE DATA FORM

Site 325 Model Stack

Date $9 / 17 / 2004$

Tester JAG/MYB/JMB

Stack Dia.

18 in.

Stack X-Area $254.5 \mathrm{in.2}$

Elevation N.A.

Distance to disturbance

Measurement units particles/ft3

Order ---.->

Traverse-->

Trial --.->
Run No. PT-2

Fan corifiguration Near Fan

Fan Setting $37.1 \mathrm{~Hz}$

Stack Temp

$66 \operatorname{deg} \mathrm{F}$

Start/End Time 10:50/13:20

Center $2 / 3$ from

1.65

Points in Center 2/3

2 to: $\frac{16}{7}$

Injection Point Near Fan, Centerline downstream 2nd

\begin{tabular}{|c|c|c|c|c|c|c|c|c|c|}
\hline \multicolumn{2}{|c|}{ | rial -..-> } & \multirow{2}{*}{\multicolumn{4}{|c|}{$\begin{array}{ccc}2 & 3 & \text { Mean } \\
\text { particles/ft3 } & \end{array}$}} & \multirow{2}{*}{\multicolumn{4}{|c|}{$\frac{2}{\text { particles/ft3 }}$}} \\
\hline Point & Depth, in. & & & & & & & & \\
\hline 1 & 0.58 & 4987 & 2769 & 2930 & 3562.0 & 2629 & 2880 & 3068 & 2859.0 \\
\hline 2 & 1.89 & 4980 & 2677 & 3068 & 3575.0 & 2772 & 3186 & 2879 & 2945.7 \\
\hline 3 & $3 . \overline{49}$ & 3190 & 3812 & 2676 & 3226.0 & 2758 & 3355 & 3548 & 3220.3 \\
\hline 4 & 5.81 & 3499 & 4058 & 2584 & 3380.3 & 2787 & 3185 & 4027 & 3333.0 \\
\hline Center & 9.00 & 4049 & 4810 & 2839 & 3899.3 & 2560 & 2986 & 4222 & 3256.0 \\
\hline 5 & 12.19 & 4318 & 3638 & 2564 & 3506.7 & 2658 & 2651 & 3828 & 3045.7 \\
\hline 6 & 14.51 & 3785 & 4313 & 3182 & 3760.0 & 3913 & 2753 & 3528 & 3398.0 \\
\hline 7 & 16.11 & 3673 & 4102 & 3688 & 3821.0 & 3755 & 2513 & 4860 & 3709.3 \\
\hline 8 & 17.42 & 3103 & 3172 & 2725 & 3000.0 & 2951 & 2999 & 5331 & 3760.3 \\
\hline Averages -... & $-\ldots>$ & 3953.8 & 3705.7 & 2917.3 & 3525.6 & 2975.9 & 2945.3 & 3921.2 & 3280.8 \\
\hline
\end{tabular}

Avg Conc $3381 \mathrm{pt} / \mathrm{ft} 3$

\begin{tabular}{|lr}
\hline All & $\mathrm{pt/ft3}$ \\
Mean & 3403.2 \\
Min Point & 2859.0 \\
Max Point & 3899.3 \\
\hline \multicolumn{2}{|c|}{$3381 \mathrm{pt}^{\mathrm{ftt} 3}$}
\end{tabular}

Generator Inlet Press

Stack Temp

Centerline vel.

Ambient pressure

Ambient humidity

Ambient temp

Back-Gd aerosol

No. Bk-Gd samples

Compressor output reg

\begin{tabular}{|c|c|c|}
\hline Start & Finish & \\
\hline 4 & 4 & psig \\
\hline 61 & 71 & $F$ \\
\hline 855 & 795.0 & $\mathrm{fpm}$ \\
\hline 29.3 & 29.258 & $\mathrm{inHg}$ \\
\hline $74 \%$ & $53 \%$ & $\mathrm{RH}$ \\
\hline 58 & 66 & $F$ \\
\hline $3,3,2,3,4,5$ & $1,3,4,3,0,3$ & $\mathrm{pt} / \mathrm{ft} 3$ \\
\hline 6 & 6 & \\
\hline 110 & 115 & psig \\
\hline
\end{tabular}

Optical Particle Counters:

\begin{tabular}{lll}
\hline Met One A2408 & S/N 96258675 & Cal 8/5/04 \\
\hline Wind 12 mph steady & \\
\hline Oil Used: FisherBrand 19 &
\end{tabular}

Oil Used: FisherBrand 19

Probe has a 4.5-in throw so is that much closer to the

stack than the port.

It appears that there was a decline and then a recovery in

concentration durring the test. See column averages.

Consistency checks: at East 1 after the East measuremements:

4430/4487/4661/4399/4338/4602

At North2: $3699 / 3692 / 3805 / 3727 / 3715 / 3804 / 3918 / 3999$

At North7: 5065/4957/5263/5052

Signature signifies compliance with

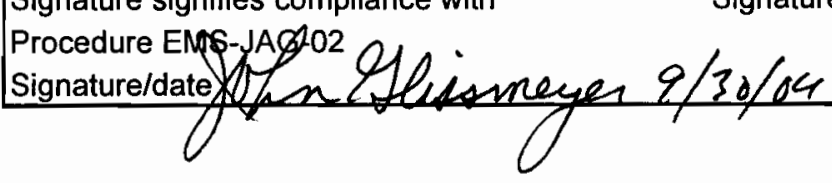

04-325 Scale-8ptpart.xls

PT-2

\begin{tabular}{|rrrr|} 
East & North & $\underline{\text { All }}$ & Normlzd \\
3595.5 & 3272.6 & 3434.02 & 3757.33 \\
245.4 & 248.8 & 290.60 & 311.44 \\
6.8 & 7.6 & $\mathbf{8 . 4 6}$ & $\mathbf{8 . 2 9}$ \\
\hline
\end{tabular}

Instuments Used:

TSI Velocity Calc Plus S/N 209060

Calib 8/25/04

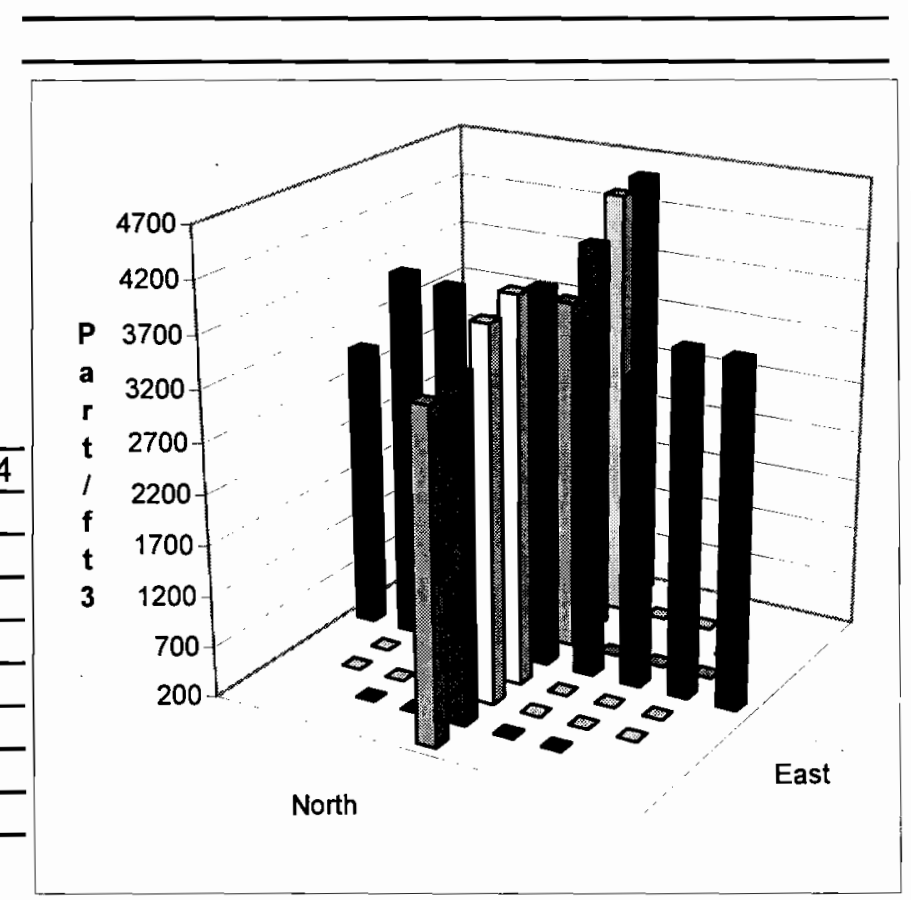


PARTICLE TRACER TRAVERSE DATA FORM

Site 325 Model Stack

Date $9 / 17 / 2004$

Tester JAG/MYB/JMB

Stack Dia.

Stack X-Area

18 in.

Elevation N.A.

Distance to disturbance

Measurement units particles/ft3

Order -...->

Traverse-->

Trial --.->
120 inches

1 st
254.5 in. 2
Run No. PT-2a

Fan configuration Near Fan

Fan Setting $37.1 \mathrm{~Hz}$

Stack Temp $66 \operatorname{deg} \mathrm{F}$

Start/End Time 10:50/13:20

Center $2 / 3$ from 1.65

Points in Center $2 / 3$ to:

to: 7

Injection Point Near Fan, Centerline downstream

\begin{tabular}{|c|c|c|c|c|c|c|c|c|c|}
\hline Trial ----> & & 1 & 2 & 3 & Mean & 1 & 2 & 3 & Mean \\
\hline Point & Depth, in. & \multicolumn{4}{|c|}{ particles/ft3 } & \multicolumn{4}{|c|}{ particles/ft3 } \\
\hline 1 & 0.58 & 4987 & 2769 & 4527 & 4094.3 & 2629 & 2880 & 3068 & 2859.0 \\
\hline 2 & 1.89 & 4980 & 2677 & 3534 & 3730.3 & 2772 & 3186 & 2879 & 2945.7 \\
\hline 3 & 3.49 & 3190 & 3812 & 4235 & 3745.7 & 2758 & 3355 & 3548 & 3220.3 \\
\hline 4 & 5.81 & 3499 & 4058 & 4313 & 3956.7 & 2787 & 3185 & 4027 & 3333.0 \\
\hline Center & 9.00 & 4049 & 4810 & 4084 & 4314.3 & 2560 & 2986 & 4222 & 3256.0 \\
\hline 5 & 12.19 & 4318 & 3638 & 3973 & 3976.3 & 2658 & 2651 & 3828 & 3045.7 \\
\hline 6 & 14.51 & 3785 & 4313 & 3856 & 3984.7 & 3913 & 2753 & 3528 & 3398.0 \\
\hline 7 & 16.11 & 3673 & 4102 & 3532 & 3769.0 & 3755 & 2513 & 4860 & 3709.3 \\
\hline 8 & 17.42 & 3103 & 3172 & 2589 & 2954.7 & 2951 & 2999 & 5331 & 3760.3 \\
\hline Averages & $>$ & 3953.8 & 3705.7 & 3849.2 & 3836.2 & 2975.9 & 2945.3 & 3921.2 & 3280.8 \\
\hline
\end{tabular}

Avg Conc $3530 \mathrm{pt} / \mathrm{ft} 3$

Generator Inlet Press

Stack Temp

Centerline vel.

Ambient pressure

Ambient humidity

Ambient temp

Back-Gd aerosol

No. Bk-Gd samples

Compressor output reg

Optical Particle Counters:

Met One A2408

S/N 96258675

\begin{tabular}{|c|c|c|}
\hline Start & Finish & \\
\hline 4 & & 4 psig \\
\hline 61 & 71 & $1 / F$ \\
\hline 855 & 795.0 & $\mathrm{fpm}$ \\
\hline 29.3 & 29.258 & $\mathrm{inHg}$ \\
\hline $74 \%$ & $53 \%$ & $\mathrm{RH}$ \\
\hline 58 & 66 & \\
\hline $3,3,2,3,4,5$ & $1,3,4,3,0,3$ & $\mathrm{pt} / \mathrm{ft}$ \\
\hline 6 & 6 & \\
\hline 110 & 115 & $5 \mathrm{psig}$ \\
\hline
\end{tabular}

$\mathrm{pt} / \mathrm{ft} 3$

3558.5

2859.0

4314.3

Wind 12 mph steady

Oil Used: FisherBrand 19

Probe has a 4.5-in throw so is that much closer to the

stack than the port.

\begin{tabular}{r|lrrrr|}
\hline Dev. from mean & Center 2/3 & East & North & All & Normlzd \\
\cline { 2 - 6 } & Mean & 3925.3 & 3272.6 & 3598.93 & 4130.79 \\
$-19.7 \%$ & Std. Dev. & 205.4 & 248.8 & 403.43 & 339.31 \\
$21.2 \%$ & COV as \% & 5.2 & 7.6 & $\mathbf{1 1 . 2 1}$ & $\mathbf{8 . 2 1}$ \\
\hline
\end{tabular}

Instuments Used:

TSI Velocity Calc Plus S/N 209060

Calib 8/25/04

(2)

(2)

Cal 8/5/04

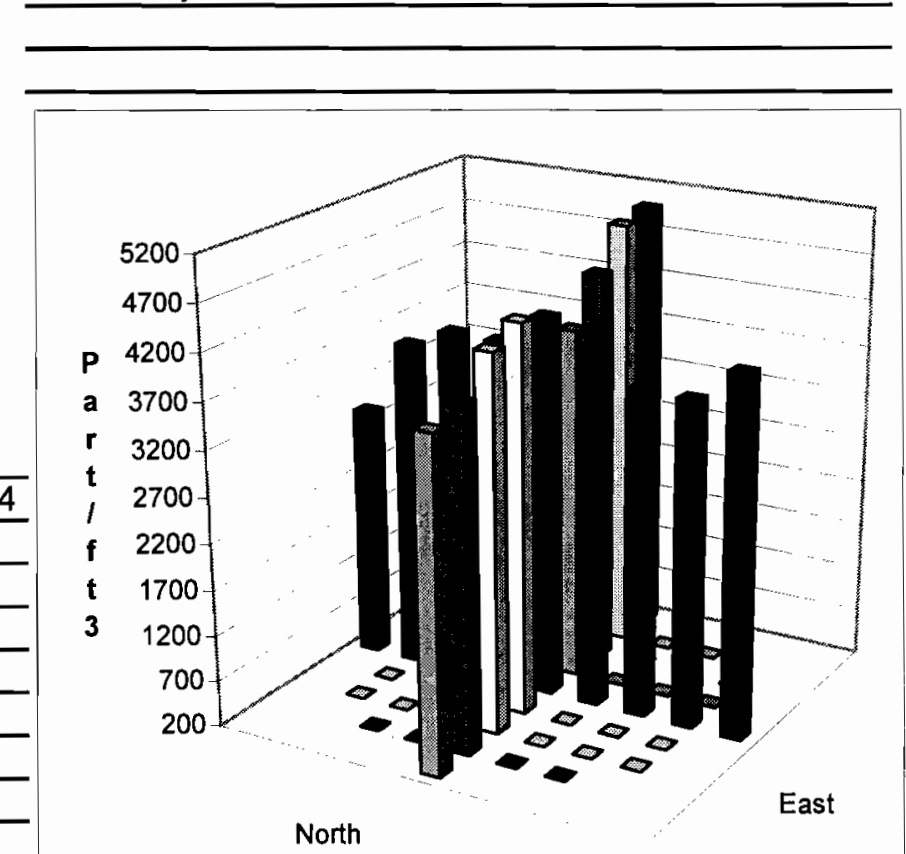

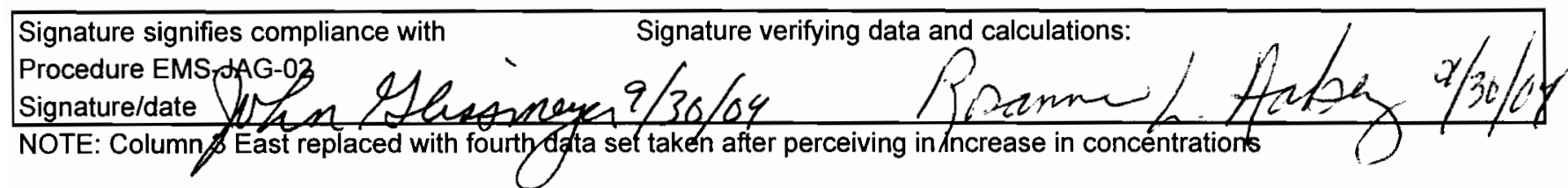

04-325 Scale-8ptpart.xls PT-2a 9/30/2004 
PARTICLE TRACER TRAVERSE DATA FORM

Site 325 Model Stack

Date $9 / 17 / 2004$

Tester JAG/MYB/JMB

Stack Dia.

18 in.

Stack X-Area $254.5 \mathrm{in.2}$

Elevation N.A.

Distance to disturbance

Measurement units particles/ft3

Order -...->

Traverse-->

Trial -...>
120 inches

2nd

Averages ----.-.-.->

Run No. PT-3

Fan configuration Far Fan

Fan Setting $\mathbf{3 7 . 1 ~ H z}$

Stack Temp

$70.5 \operatorname{deg} F$

Start/End Time 1415/1620

Center 2/3 from

Points in Center 2/3

Injection Point Dwnstrm of far fan, centerline

\begin{tabular}{|c|c|c|c|c|c|c|c|c|c|}
\hline Trial ---.> & & 1 & 2 & 3 & Mean & 1 & 2 & 3 & Mean \\
\hline Point & Depth, in. & \multicolumn{4}{|c|}{ particles/ft3 } & \multicolumn{4}{|c|}{ particles/ft3 } \\
\hline 1 & 0.58 & 765 & 651 & 648 & 688.0 & 665 & 519 & 486 & 556.7 \\
\hline 2 & 1.89 & 715 & 646 & 688 & 683.0 & 625 & 542 & 657 & 608.0 \\
\hline 3 & 3.49 & 642 & 572 & 556 & 590.0 & 542 & 712 & 844 & 699.3 \\
\hline 4 & 5.81 & 533 & 571 & 510 & 538.0 & 772 & 477 & 812 & 687.0 \\
\hline Center & 9.00 & 472 & 563 & 657 & 564.0 & 767 & 521 & 836 & 708.0 \\
\hline 5 & 12.19 & 651 & 533 & 548 & 577.3 & 757 & 557 & 808 & 707.3 \\
\hline 6 & 14.51 & 632 & 600 & 500 & 577.3 & 769 & 559 & 778 & 702.0 \\
\hline 7 & 16.11 & 549 & 617 & 491 & 552.3 & 739 & 556 & 762 & 685.7 \\
\hline 8 & 17.42 & 518 & 541 & 375 & 478.0 & 818 & 766 & 778 & 787.3 \\
\hline Averages & $\ldots$ & 608.6 & \begin{tabular}{|l|}
588.2 \\
\end{tabular} & 552.6 & 583.1 & 717.1 & 578.8 & 751.2 & 682.4 \\
\hline
\end{tabular}

\begin{tabular}{|llr|lrrrr|r|}
\hline All & pt/ft3 & Dev. from mean & Center 2/3 & East & North & All & Normlzd \\
Mean & 632.7 & & Mean & 583.1 & 685.3 & 634.24 & 708.68 \\
Min Point & 478.0 & $-24.5 \%$ & Std. Dev. & 47.3 & 35.2 & 66.47 & 52.82 \\
Max Point & 787.3 & $24.4 \%$ & COV as \% & 8.1 & 5.1 & $\mathbf{1 0 . 4 8}$ & $\mathbf{7 . 4 5}$ \\
\hline
\end{tabular}

Avg Conc $632 \mathrm{pt} / \mathrm{ft} 3$

Generator Inlet Press

Stack Temp

Centerline vel.

Ambient pressure

Ambient humidity

Ambient temp

Back-Gd aerosol

No. Bk-Gd samples

Compressor output reg

Optical Particle Counters:

\begin{tabular}{lll}
\hline Met One A2408 & S/N 96258675 & Cal 8/5/04 \\
\hline
\end{tabular}

SW wind $10 \mathrm{mph}$ at start

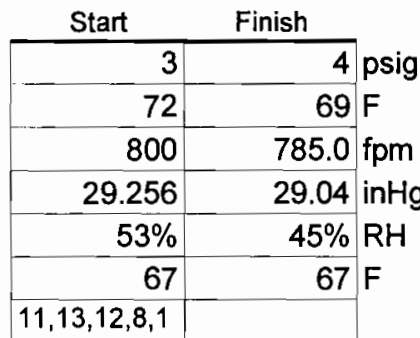

Instuments Used:

TSI Velocity Calc Plus S/N 209060

Calib $8 / 25 / 04$

SE wind $12 \mathrm{mph}$ at end

Oil Used: FisherBrand 19

Probe has a 4.5-in throw so is that much closer to the

stack than the port.

Pretest consistency checked at N4 point:

$718,672,642,672,739$
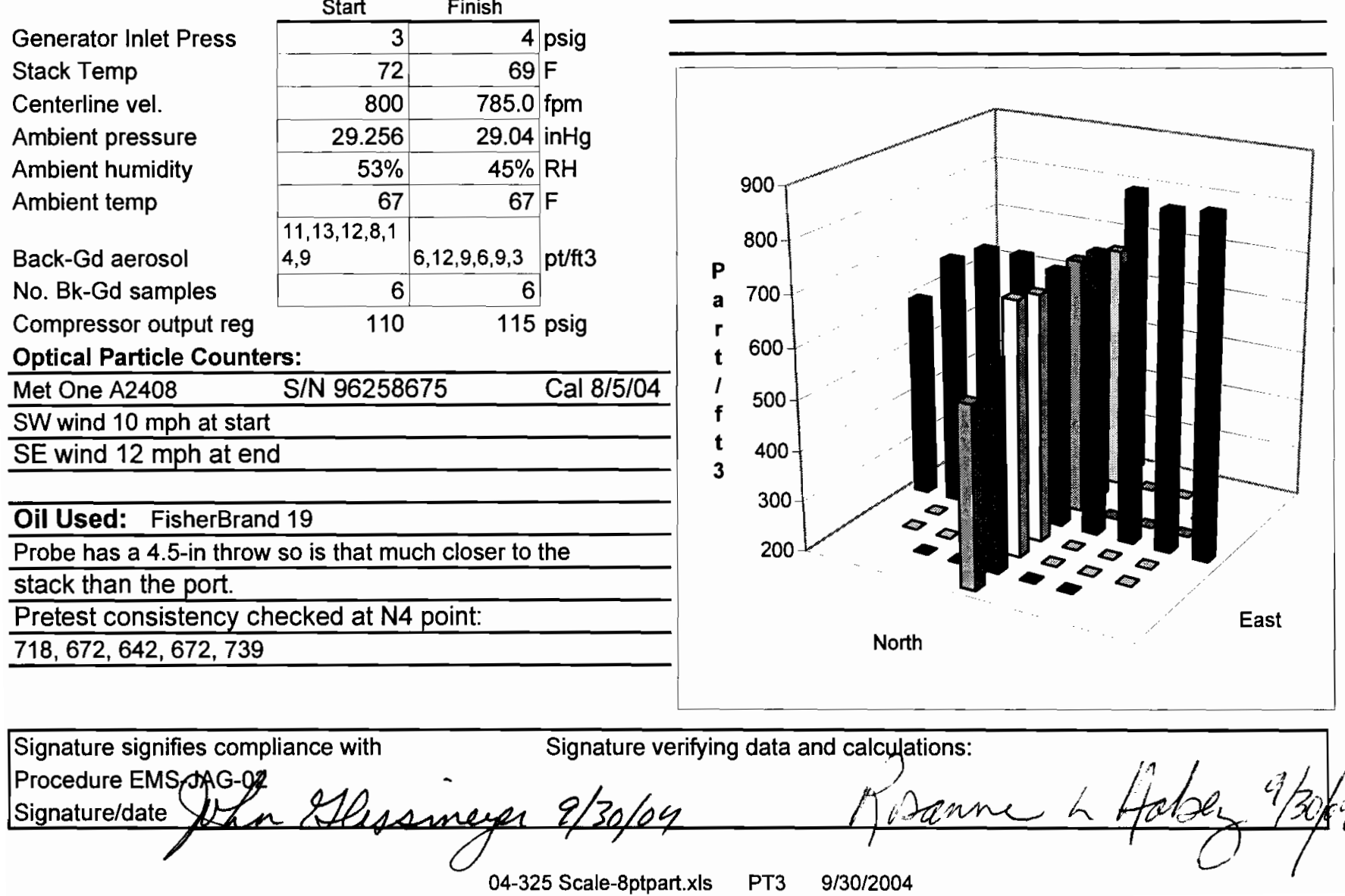

PT3

9/30/2004

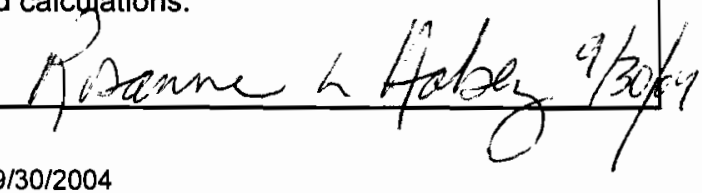




\section{Test Instruction}

\begin{tabular}{l|l|l}
\hline $\begin{array}{l}\text { Project: 325 Stack Sampler } \\
\text { Qualification }\end{array}$ & Date: August 16, 2004 & Work Package: F59676 \\
\hline
\end{tabular}

Tests: Tracer Particle Uniformity in 325 Model Stack, 1 Electric Fan Configuration

Staff: John Glissmeyer, Dave Douglas, Marcel Ballinger, Matthew Barnett

\section{Reference Procedures:}

1. Procedure EMS-JAG-02, Rev. 1, Test to Determine Uniformity of a Particulate Aerosol at a Sampler, May 24, 2000

2. Operating Manual for Met-One Optical Particle Counter (OPC), Model A2408

Equipment:

1. 325 Model Stack and inspected work platforms. Fans will be in positions EF1, EF4.

2. Vacuum pump oil, oil mist generator, air lines, regulator, precision pressure gauge compressed air source

3. Oil mist injection probe, OPC sample probes, probe/stack couplers, tape measure, marking pen

4. OPC with computer (optional) and link

5. Velocity measurement device (optional) for verifying stack flow

Safety Considerations:

Observe the applicable Job Hazard Analysis for the project

Instructions:

1. Verify training on the procedure and that instrumentation is within calibration

2. Obtain climatic information from the Hanford Weather Service, phone 373-2716 or http://etd.pnl.gov:2080/HMS/lastob.htm

3. Mark the completion of each step on the field copy of the procedure. Mark-out those steps not applicable to this stack.

4. Install equipment as directed in the procedures. Only the mobile OPC will be used. The aerosol delivery line should be as vertical as possible to avoid oil accumulation.

5. Use a sliding platform for the OPC and clamp the probe so both the OPC and probe move together.

6. Mark sampling probe for the measurement points shown on the data sheet.

7. Verify that stack flow is about $1350-1450 \mathrm{cfm}$.

8. Initially set the injection system input psi at 5 and vary to obtain particle counts at the sampling ports that are about 10 times background for 10-micron particles.

9. Monitor the flowrate on the OPC. Record data on copies of the attached the data sheet. Diagram mounting fixtures and retain assembly for any subsequent re-tests

10. Conduct one or more tracer mixing tests at the following sets of conditions:

\begin{tabular}{|l|l|l|}
\hline Downstream of Fan & Injection Positions & Possible Runs \\
\hline EF1 & Center & PT 1 \\
\hline EF4 & Center & PT 2 \\
\hline $\begin{array}{l}\text { Worst case fan position from either this } \\
\text { or gas tracer tests }\end{array}$ & Center & PT 3 \\
\hline
\end{tabular}

\section{Desired Completion Date: 08/27/04}

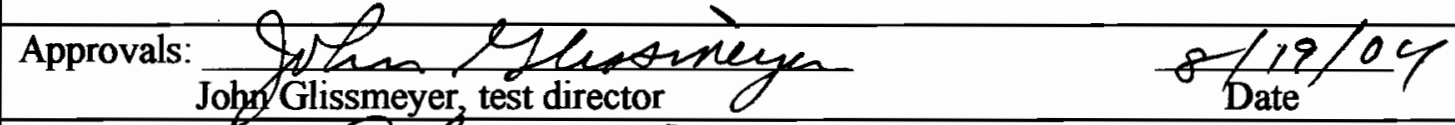

Test Completed by: Sate:

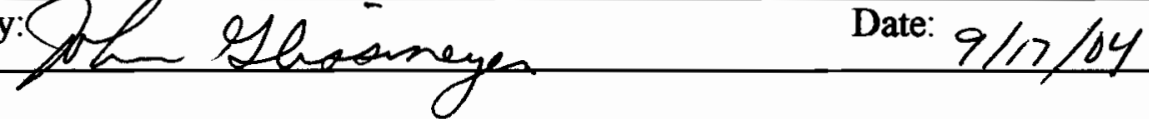


Title: Test to Determine Uniformity of a Tracer Aerosol at a Sampler Probe

\begin{tabular}{|c|c|c|c|}
\hline \multicolumn{4}{|l|}{ PNNL Operating Procedure } \\
\hline $\begin{array}{l}\text { Title: Test to Determine } \\
\text { Uniformity of a Tracer } \\
\text { Aerosol at a Sampler } \\
\text { Probe }\end{array}$ & $\begin{array}{l}\text { Org. Code: } \\
\text { Procedure No.: } \\
\text { Rev. No.: }\end{array}$ & $\begin{array}{l}\text { D9T99 } \\
\text { EMS-JAG-02 } \\
1\end{array}$ & \\
\hline Work Location: General & Effective Date: & May 24, 2000 & \\
\hline Author: John A. Glissmeyer & Supersedes Date: & November 10,199 & \\
\hline $\begin{array}{l}\text { Identified Hazards: } \\
\square \text { Radiological } \\
\square \text { Hazardous Materials } \\
\text { Physical Hazards } \\
\square \text { Hazardous Environment } \\
\square \text { Other: }\end{array}$ & $\begin{array}{l}\text { Identified Use Cat } \\
\square \text { Mandatory Use } \\
\square \text { Continuous Use } \\
\text { Reference Use } \\
\square \text { Information Use }\end{array}$ & gory: & \\
\hline \multicolumn{4}{|c|}{ Are One-Time Modifications Allowed? $\quad$ Yes $\square$ No } \\
\hline Person Signing & Sigr & ature & Date \\
\hline \multicolumn{4}{|l|}{$\begin{array}{l}\text { Technical review: } \\
\text { James L. Huckaby }\end{array}$} \\
\hline \multicolumn{4}{|l|}{$\begin{array}{l}\text { Project Manager: } \\
\text { John Glissmeyer }\end{array}$} \\
\hline \multicolumn{4}{|l|}{$\begin{array}{l}\text { Line Manager: } \\
\text { James Droppo }\end{array}$} \\
\hline \multicolumn{4}{|l|}{ Concurrence: } \\
\hline $\begin{array}{l}\text { Quality Engineer: } \\
\text { Thomas G. Walker }\end{array}$ & & & \\
\hline
\end{tabular}




\begin{tabular}{l|ll}
\hline PNNL Operating Procedure & $\begin{array}{l}\text { Rev. No. 1 } \\
\text { Org. Code: D9T99 }\end{array}$ & $\begin{array}{l}\text { Page 2 of 15 } \\
\text { Procedure No.: EMS-JAG-02 }\end{array}$
\end{tabular}

Title: Test to Determine Uniformity of a Tracer Aerosol at a Sampler Probe

\subsection{Purpose}

The performance of new stack sampling systems must be shown to satisfy the requirements of 40 CFR 61, Subpart H, "National Emission standards for Emissions of Radionuclides Other than Radon from Department of Energy Facilities." This regulation governs portions of the design and implementation of effluent air sampling. The stack sampler performance is adequately characterized when potential contaminants in the effluent are of a uniform concentration at the sampling location (plane), and line losses are within acceptable limits. This procedure determines whether the concentration of aerosol particulate contaminants is uniformly distributed in the area of the sampling probe. Other procedures address flow angle, uniformity of gas velocity, and uniformity of gas contaminants. A contaminant concentration that is uniform at the sampling plane enables the extraction of samples that represent the true emission concentration.

The uniformity is expressed as the variability of the measurements about the mean. This is expressed using the relative coefficient of variance (COV), which is the standard deviation divided by the mean and expressed as a percentage. The lower the COV value, the more uniform the particle concentration. The acceptance criterion is that the COV of the measured particle concentrations be $\# 20 \%$ across the center two-thirds of the area of the stack.

\subsection{Applicability}

This procedure can be used in the field or on modeled stacks to determine whether air-sampling probes can collect representative samples under normal operations. The tests are applicable to effluent stacks or ducts within the following constraints:

- The aerosol particulate tests are generally limited to stacks with flowrates greater than 50 cubic feet per minute range. The upper bound of flowrate is determined by the output capacity of the aerosol generator, the background reading for particulate aerosols, and the operational detection range of the optical particle counters.

- Environmental constraints - optical particle counters will require the use of a controlled temperature environment to maintain the equipment above 55 degrees Fahrenheit.

\subsection{Prerequisites and Conditions}

Conditions and concerns that must be satisfied before sampling are listed below:

- Safety glasses and hard toed or substantial shoes are required in work areas.

- Test ports for tracer injection and sampling.

- Properly constructed and inspected work platforms may be needed to access the test ports.

- Scaffold-user or fall protection training may be required to access the sampling ports of the stack.

- Alcohol may be used for equipment cleanup. A flammable equipment storage cabinet is required to hold chemicals. Material Safety Data Sheets must be provided. 


\begin{tabular}{l|ll}
\hline PNNL Operating Procedure & $\begin{array}{l}\text { Rev. No. 1 } \\
\text { Org. Code: D9T99 }\end{array}$ & Page 3 of 15 \\
& Procedure No.: EMS-JAG-02
\end{tabular}

Title: Test to Determine Uniformity of a Tracer Aerosol at a Sampler Probe

- Air pressure (up to about $75 \mathrm{psi}$ ) is used to aerosolize oil into fine particles. Knowledge of the use and operation of pressurized air-lines, and the careful observations of any buildup of oil mist outside of the generator is essential to prevent exceeding American Conference of Governmental Industrial Hygienists (ACGIH) levels listed below.

- Knowledge of the setup, use of, and operation of flowmeters, particle counters, and computers is essential.

- A job-hazards analysis may be required in certain cases.

\subsection{Precautions and Limitations}

\section{Caution: The ACGIH 8-hour time-weighted average limit for human exposure to mineral oil mist is 5 $\mathrm{mg} / \mathrm{m}^{3}$. It is odorless.}

During tests of stacks with high flowrates, oil droplets will be injected into the base of the stack to overcome the large dilution factor needed to detect selected particles at the sampling ports above. The potential is present for a buildup of oil mist to occur outside of the aerosol generator that could approach the $5 \mathrm{mg} / \mathrm{m}^{3}$ caution level. The undiluted mist is heavier than air, so it may accumulate in confined spaces and in low areas if allowed to escape. Visual inspections of the delivery system will be made at least daily to prevent such an occurrence.

Access to the test ports may require the use of scaffolding or manlifts, either of which will necessitate special training for sampling personnel and any observers. The training requirements will be indicated in the job hazard analysis.

The test may be invalid if the ending ambient concentration of mist is elevated above that observed at the start of the test. This would indicate poor dispersion away from the test site caused by recirculation of the tracer to the inlet of the fan and will only occur if the stack exhaust point is in view of and is reasonably close to the fan inlet. This may result in a false indication of good mixing.

\subsection{Equipment Used for Stack Measurements}

Specific calibration check concentration levels, probe dimensions, measurement grids, flowrates, and other special requirements will be provided in the specific Test Instruction. Exhibit A provides a typical layout for the test setup. The following are essential items of equipment:

- Vacuum pump oil

- Oil mist generator

- Compressed air, compressed air hoses, and precision air regulators

- Oil mist injection probe

- Aerosol sampling probes

- Mechanism for accurate placement of sampling probe 


\begin{tabular}{|l|ll}
\hline PNNL Operating Procedure & $\begin{array}{l}\text { Rev. No. l } \\
\text { Org. Code: D9T99 }\end{array}$ & $\begin{array}{l}\text { Page 4 of 15 } \\
\text { Procedure No.: EMS-JAG-02 }\end{array}$ \\
\hline
\end{tabular}

Title: Test to Determine Uniformity of a Tracer Aerosol at a Sampler Probe

- Optical particle counters

- Computers linked to particle counters

- Velocity flow measurement meter.

Two optical particle counters (OPCs) may be used simultaneously to count particles that are approximately in the 10-micron size range. A mobile OPC is designated to make point-by-point measurements in the orthogonal traverses. An optional reference OPC may be used to note trends in aerosol generator output over time and to validate the mobile sampler results. The operation of the reference OPC, at some fixed position in the stack, may be contingent on whether a suitable port is available on the test stack.

The counters, rechecked annually for calibration by the manufacturer, are synchronized for time, sample mode, flow, and count range to monitor their field performance. The absolute calibration of the OPCs is not as important as the general response because the concentration data are used in a relative manner in calculating the COV and in plotting the concentrations at the measurement points.

The aerosol generator siphons oil from a reservoir and forces the air/oil mixture through a spray nozzle to produce polydisperse particles. Non-hazardous oil with a low vapor pressure (such as Fisherbrand 19 vacuum pump oil) should be used in the reservoir. The quantity of aerosol generated is controlled by the amount of compressed air pressure, which should be filtered and controlled by a precision regulator. The nozzle is mounted in a large diameter, clear-plastic pipe (4-inches diameter or larger) so the output level can be observed. The aerosol generator output should connect to an injection tube with an inside diameter of at least 0.5 inches to minimize collisions with the inner wall of the tubing. Optimal operation depends on uniformly "wetting" the inner surfaces of the generator and transfer tubes; thus, a warm up period of up to $1 / 2$ hour is needed for a constant aerosol output.

\subsection{Work instructions for Setup, Measurements, and Data Reduction}

The steps taken to set up, configure, and operate the stack fans and test equipment are listed. Based on previous field measurements, the steps are ordered to achieve maximum efficiency in the testing. In addition to these steps, the test instruction illustrated in Attachment A will provide specific details and operating parameters. 
Title: Test to Determine Uniformity of a Tracer Aerosol at a Sampler Probe

\subsection{Preliminary Steps:}

6.1.1 Provide essential supplies at the sampling location (particulate generation equipment, supply air and regulators, fittings and probe-port couplers, marking pens, data sheets, writing and probe-supporting platforms).

\footnotetext{
quylibloy
}

6.1.2 Fill in test information on dataform.

myb

6.1.3 Observe the current flow setting for the test stack and record $9 / 16 / 04$ on the data sheet.

6.1.4 Obtain barometric, temperature, and relative humidity 9/16/04 information for the particle counter location.

MMYB

6.1.5 Measure the stack centerline air velocity in the sampling $9 / 16 / 04 \quad$ plane usir

6.1.6 Mark the sampling probe with a permanent marker so the inlet can be placed at each successive measurement point.

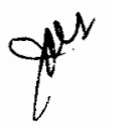

Note: Sampling plane traverse points. Use the grid of measurement points provided with the test's instruction and dataform. This is usually the same as used for the velocity uniformity test. A center point is included as a common reference and for graphical purposes. The layout design divides the area of the sampling plane so that each point represents approximately an equal-sized area

6.1.7 Couple the OPCs and probes to the stack sampling ports according to the illustration in Exhibit A. 


\begin{tabular}{l|ll} 
PNNL Operating Procedure & $\begin{array}{l}\text { Rev. No. 1 } \\
\text { Org. Code: D9T99 }\end{array}$ & $\begin{array}{l}\text { Page } 6 \text { of 15 } \\
\text { Procedure No.: EMS-JAG-02 }\end{array}$ \\
\hline
\end{tabular}

Title: Test to Determine Uniformity of a Tracer Aerosol at a Sampler Probe

Note: The sampling equipment consists of stainless steel probes with $3 / 4$ outside diameter and thinwall tubing with sufficient length to reach across the inside diameter of the stack while allowing for fittings. The sampling probe should have gradual $90^{\circ}$ bends to minimize the inertial impact of particles with inner walls at bends, and the open end of the tube should face downward or into the flow in the stack. The outlet end of the probe should terminate at the OPC inlet. Minimize tubing length to minimize particle losses.

The sampling probes for both OPCs should be similar and of a simple design. The elevation of the intake nozzle of the traversing unit should be approximately in the same as the sampling plane. The intake nozzle for the reference unit may be located anywhere within the stack at an elevation near that of the sampling plane; however, the two probes should not interfere with each other, either physically or by causing flow disturbances for each other. The intake nozzles may be of sub-isokinetic or of shrouded design to optimize the collection of 10-micron particles.

The aerodynamic characteristics of the probes for both OPCs should be the same so that they have similar line-loss (penetration) values. For optimal particle collection, the probes should be of a fixed and rigid configuration. The mobile $\mathrm{OPC}$ with its attached probe should be mounted together on a sliding platform to move as a unit along the axis of the sampling port.

\title{
6.1.8 Turn-on the mobile $\ln y \mathcal{B}$
} Mngs
$9 / 16 / 04$ optical particle counters.

Note: Ensure that internal air circulation fans in the OPCs are on and that the sample probes are tightly connected to and are directly above or apart from the OPC sample inlet openings. Also ensure that the sliding platform supporting the mobile sampler is aligned for easy, free movement at the correct height for its stack port.

\author{
6.1.9 Program and synchronize the OPCs for \\ myb \\ $9 / 16 / 04$ \\ - 60-second samples \\ - 9- to 11-micron particle counting \\ - the current time \\ - cumulative counting mode.
}


\begin{tabular}{l|ll} 
PNNL Operating Procedure & Rev. No. 1 & Page 7 of 15
\end{tabular}

Org. Code: D9T99

Procedure No.: EMS-JAG-02

Title: Test to Determine Uniformity of a Tracer Aerosol at a Sampler Probe

\subsection{Daily Particulate Background Concentration Measurement}

6.2.1 At the beginning of each sampling day before starting the

$y_{9 / 6 / 6} / 04$ aerosol generator, obtain at least six consecutive

background readings for both mobile and reference OPCs.

6.2.2 Record these readings on the data sheet and in logbols $9 / 15 / 04$

(4) 4 B $/ 6 / 04$ designated for the tests.

6.2.3 Start and run the aerosol generator for approximately

919.8 30 minutes to stabilize its output.

\subsection{Particle Injection and Sample Collection}

The injection equipment includes an air regulator, a precision air pressure gauge, and other components described in Section 5. The 3/4-inch (OD) (or larger) injection probe with a 90E bend (with an approximately 3 -inch radius of turn) will inject aerosol particles in the direction of emission flow. The connections and fittings should be checked to ensure that they are secure and leak free.

Note: Location of the Injection Point

Injection plane -- The tests are repeated using the centerpoint as the aerosol release point.

6.3.1 Position the injection probe, according to the test

$\min _{9 / 6} / 0.1$ instruction.

6.3.2 Start injection of the aerosol and adjust the flowrate to the m $4 B$ input capabilities of the OPCs.

$9 / 16 / 04$

Note: Aerosol injection is not precisely controlled. At air pressure readings above about 10 psi for the specific PNNL generator used, a dense oil mist is created in the generator and is available for injection. However, if the back-pressure, caused by a high rate of airflow past the port in the stack, at the injection port is high, carrier air may be required to inject the aerosol into the base of the stack. Under these conditions, the overall aerosol output will be low (less than perhaps 200 particles measured at the counter).

In contrast, if there is little back-pressure, most of the generated aerosol, minus that lost from interactions with internal generator system and line walls, becomes available for injection. Here the output will be high (hundreds to thousands of particles injected per minute). 
Title: Test to Determine Uniformity of a Tracer Aerosol at a Sampler Probe

Note: The OPC draws air from the stack, via the sample probe, at a fixed rate (one cubic foot per minute). Within the OPC, the air stream with particles passes through a laser beam where the particles are counted and placed in six size categories. In the less than 0.5-micron category, several hundred thousand differential counts are typical; but in the 9- to 11-micron category, oil mists greater than about $3,000 \mathrm{cpm}$ cause a sensor overload condition. Thus, at the OPC, the flow rate is fixed, and a ceiling exists on the measurement of particles. Essentially, there is no adjustment of particle counting capability at the OPC, and the aerosol generator becomes the controlling factor for particulate output.

\subsubsection{Record the initial}

- injection system dispersion pressure in psi

- flowrate for the mobile OPC my

- centerline flow velocity for the test stack.

6.3.4 On the data sheet, label the columns of data according to the $\ln _{9 / 16 / 04}$ directions of the traverses. m $43_{9}^{6.3 .5} / 6 / 04$

6.3.5 Verify that the directional orientations and the numbered

6.3.6 Position the OPC and sample probe at each measurement $m y^{B} / / b / d y$ point in succession, and record the reading on the data form.

Note: In each test, the measurement at each point is the average of three readings. The repeats are made as three separate runs and not as three consecutive measurements at each point. 


\begin{tabular}{|l|ll}
\hline PWWT Operating Procedure & $\begin{array}{l}\text { Rev. No. 1 } \\
\text { Org. Code: D9T99 }\end{array}$ & $\begin{array}{l}\text { Page 9 of 15 } \\
\text { Procedure No.: EMS-JAG-02 }\end{array}$ \\
\hline
\end{tabular}

Title: Test to Determine Uniformity of a Tracer Aerosol at a Sampler Probe

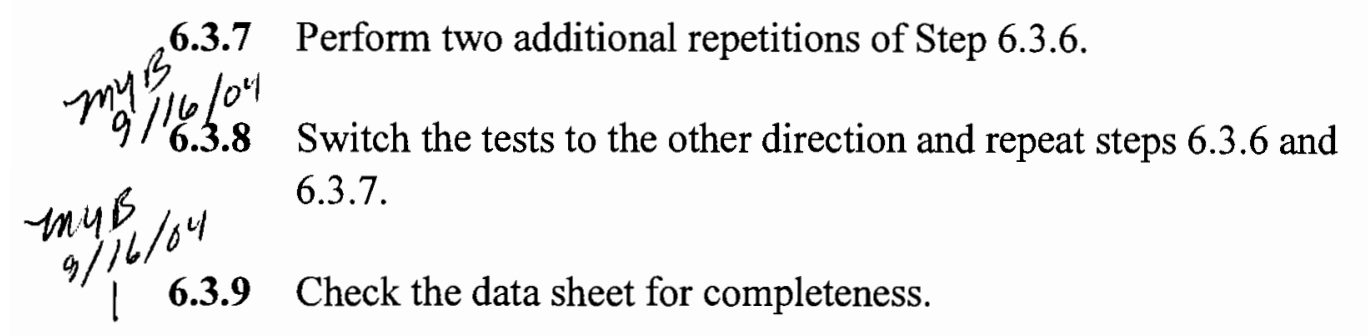

6.3.10 Record the final

$-1 \times 3$
$9 / 16 / 04$

- injection system dispersion pressure in psi
- flowrate for the mobile $M y B$

$\operatorname{mp}_{9 / 6 / 04} 6.3 .11$ Shut off the air pressure to the aerosol generator.

$\begin{array}{ll}\text { 6.3.12 } & \text { Continue operation of the OPCs for several minutes to purge any } \\ 9 / 16 / 04 & \text { remaining test aerosol from the stack. }\end{array}$

6.3.13 Measure the centerline background particulate

$\begin{array}{ll}M y^{B} 3 / 16 / 04 & \text { concentrations at the mobile monitor and record the levels } \\ \text { on the data sheet. }\end{array}$

$\begin{array}{ll}\text { m } & 6.3 .14 \\ 9 / 16 / 04 & \text { Record any climatic conditions that have changed on the }\end{array}$

$\begin{array}{ll}6.3 .15 & \text { Measure the final centerline stack velocity flow on the data } \\ 9 / 16 / 64 & \text { sheet. }\end{array}$
Imy 6.3 .16 Record any deviations from the above procedure on the data $9 / 16 / 04$ sheet.

6.3.17 Repeat steps 6.3.1 to 6.3.16 for each run as indicated in the $\operatorname{lop}_{q / 17 / 64}^{1}$

\subsection{Data Recording and Calculations}

Prepare the electronic data sheet on which to enter particle-count readings and other information relevant to the test (see test instruction). 


\section{Title: Test to Determine Uniformity of a Tracer Aerosol at a Sampler Probe}

$\Phi_{q}^{8}+\left.17\right|^{4}$

6.4.1 Review the raw data sheets for completeness.

6.4.2 Enter the data into the electronic data sheet.

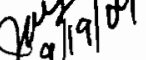

6.4.3 Calculate the COV for the run.

Note: The EXCEL datasheet shown as Attachment $C$ is set up to calculate the $\mathrm{COV}$ for each particulate concentration traverse using the average concentration data from all points in the inner two-thirds of the cross section area of the plane (including the center point).

6.4.4 Compare the observed COV for each run to the acceptance criterion.

Note: The test is acceptable if the COV is $\# 20 \%$ for the inner two-thirds of the stack diameter, and if no point differs from the mean by more than $30 \%$. This is determined by inspecting the average concentration at each measurement point. The $\mathrm{COV}$ is 100 times the standard deviation divided by the mean. attesting to its validity.

Note: A separate datasheet will be provided and signed-off for each test. 


\begin{tabular}{|l|ll|}
\hline PNNL Operating Procedure & $\begin{array}{l}\text { Rev. No. 1 } \\
\text { Org. Code: D9T99 }\end{array}$ & $\begin{array}{l}\text { Page 11 of 15 } \\
\text { Procedure No.: EMS-JAG-02 }\end{array}$ \\
\hline
\end{tabular}

Title: Test to Determine Uniformity of a Tracer Aerosol at a Sampler Probe

\section{Exhibits/Attachments}

Exhibit A - Overview of Stack and Injection Setup and Particle Counters

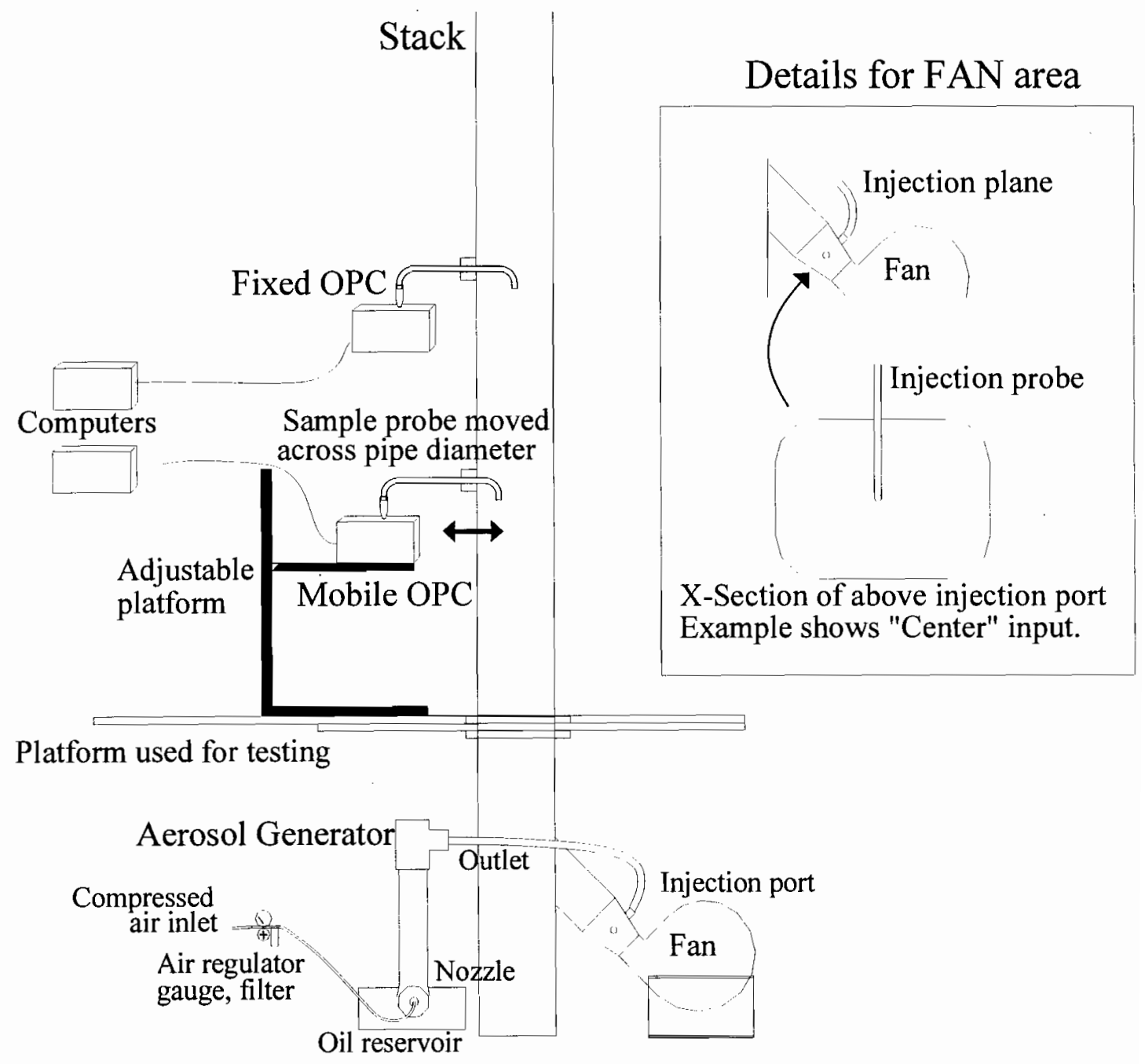




\begin{tabular}{|l|ll|}
\hline PNNL Operating Procedure & $\begin{array}{l}\text { Rev. No. 1 } \\
\text { Org. Code: D9T99 }\end{array}$ & $\begin{array}{l}\text { Page 12 of 15 } \\
\text { Procedure No.: EMS-JAG-02 }\end{array}$ \\
\hline \multicolumn{2}{|c|}{ Title: Test to Determine Uniformity of a Tracer Aerosol at a Sampler Probe } \\
\hline
\end{tabular}

Exhibit B - Illustrative Data Collection Sheet

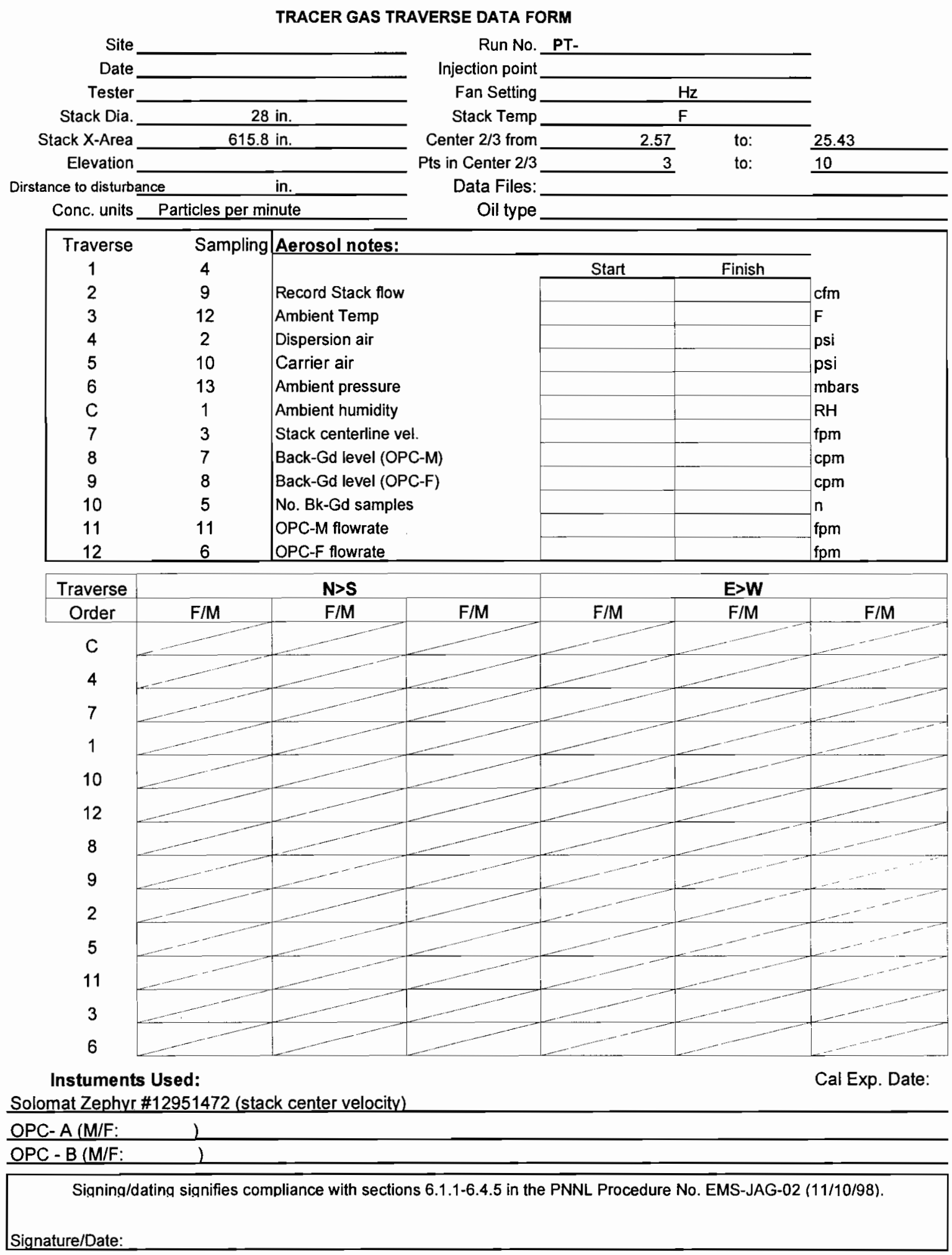




\begin{tabular}{l|ll} 
PNNL Operating Procedure & $\begin{array}{l}\text { Rev. No. 1 } \\
\text { Org. Code: D9T99 }\end{array}$ & $\begin{array}{l}\text { Page 13 of 15 } \\
\text { Procedure No.: EMS-JAG-02 }\end{array}$
\end{tabular}

\section{Title: Test to Determine Uniformity of a Tracer Aerosol at a Sampler Probe}

Exhibit C - Illus trative Data Reporting Form

PARTICULATE TRAVERSE DATA REPORT FORM

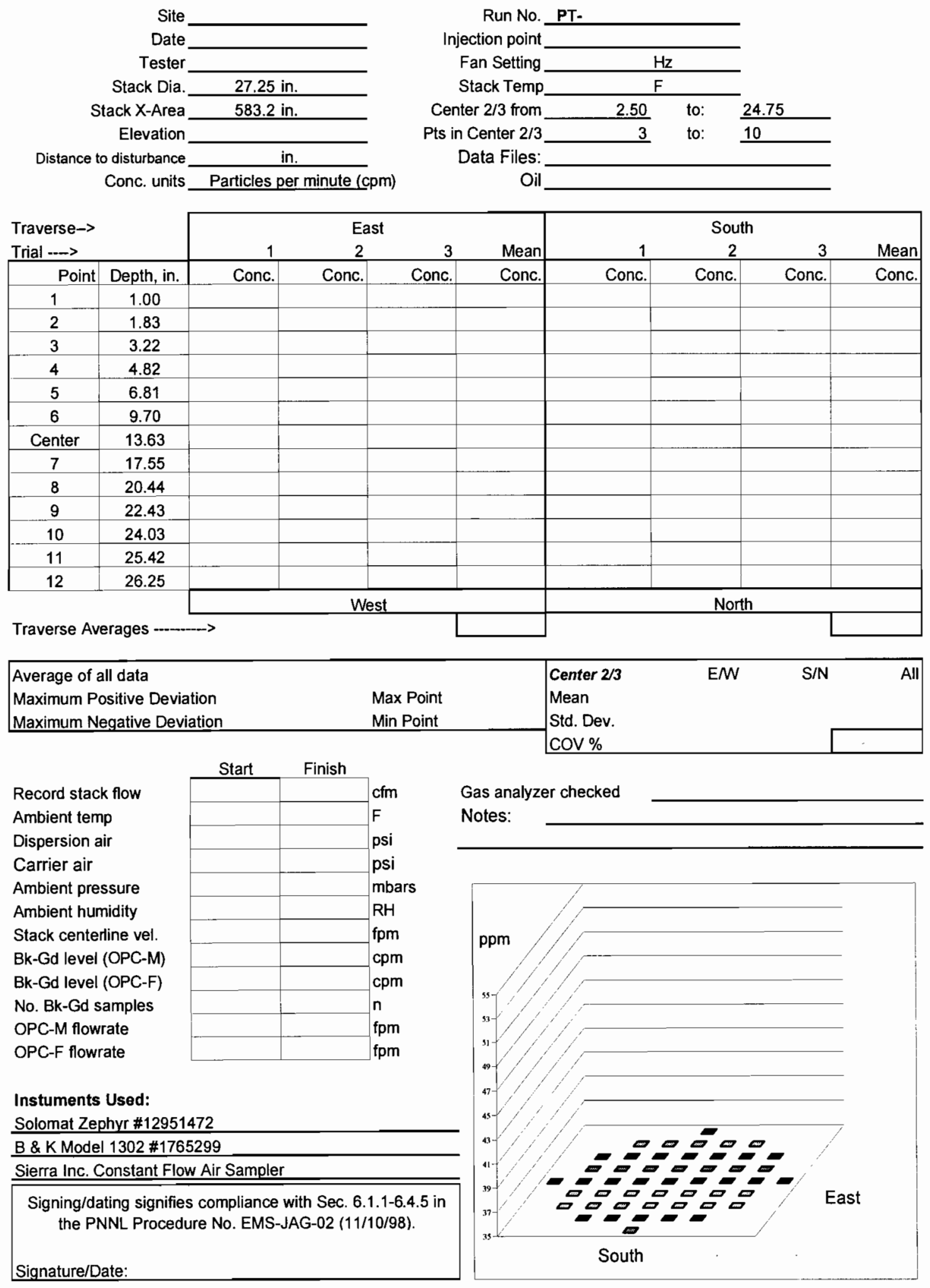


Title: Test to Determine Uniformity of a Tracer Aerosol at a Sampler Probe

\begin{tabular}{|l|l|l|}
\hline \multicolumn{3}{|c}{ Test Instruction } \\
\hline $\begin{array}{l}\text { Project: Canister Storage } \\
\text { Stack Qualification, 29303 }\end{array}$ & Date: November 10, 1998 & Work Package: K97052 \\
\hline
\end{tabular}

Tests: Tracer Gas Uniformity of Full-Scale Stack

Staff: David Maughan, John Glissmeyer

Reference Procedures:

1. Procedure EMS-JAG-02, Rev. 0, Test to Determine Uniformity of a Particulate Aerosol at a Sampler, Nov. 10, 1998

2. Operating Manual for Met-One Optical Particle Counter (OPC), Model A2408 Equipment:

1. Canister Storage Stack and inspected work platforms

2. Vacuum pump oil, oil mist generator, air lines, regulator, precision pressure gauge

3. Oil mist injection probe, OPC sample probes, probe/stack couplers

4. OPCs with computers and links

5. Velocity measurement device

Safety Considerations:

Review and observe the applicable Duke Job Hazard Analysis for the project

Instructions:

1. Verify training on the procedure and that instrumentation is within calibration

2. Obtain Fisherbrand 19 Mechanical Pump Fluid

3. Obtain climatic information from the Hanford Weather Service, phone 373-2716 or http://etd.pnl.gov:2080/HMS/lastob.htm

4. Install equipment as directed in the procedures

5. Mark sampling probe for the measurement points shown on the data sheet

6. Verify that stack flow is about the target flowrate $9000(2232 \mathrm{fpm})$

7. Initially set the injection system input psi at 5 and vary to obtain particle counts at the sampling ports that are about 10 times background for 10 -micron particles.

8. Set the sampler flowrate at approximately $10 \mathrm{lpm}$

9. Conduct one or more tracer mixing tests at the following sets of conditions:

$\frac{\text { Stack Flow }}{\text { Normal }} \quad \frac{\text { Injection point at duct from fan to stack }}{\text { Centerline }}$

(The injection plane should be at the fittings provided in the rectangular discharge of the fan)

10. Record data on copies of the attached the data sheet

11. Repeat the test

12. Diagram mounting fixtures and retain assembly for any subsequent re-tests Desired Completion Date: 11/30/98

Approvals:

John Glissmeyer, Project Manager

Date

Test completed by: Date:



Appendix G

Calculations for Scale Model Criteria 



\section{Calculations for Scale Model Criteria}

Reynolds Number Calculations

$\mathrm{Re}=\mathrm{rho}^{*} \mathrm{~V} * \mathrm{D} / \mathrm{u}$

Where Re $=$ Reynolds Number

$\mathrm{D}=$ hydraulic diameter $=$ diameter for cylinders

rho = air density $=1.1769 \mathrm{~kg} / \mathrm{m} 3$

$\mathrm{u}=$ air viscosity $=1.85 \mathrm{E}-05 \mathrm{~Pa} \mathrm{~s}$

Density and viscosity of air at 300K from Fundamentals of Momentum, Heat, and Mass Transfer, Welty, Wicks, and Wilson 1976

$\mathrm{V}=$ velocity $=\mathrm{Q} / \mathrm{A}$ in $\mathrm{m} / \mathrm{s}$

$\mathrm{Q}=$ stack flow rate $\mathrm{m} 3 / \mathrm{s}$

$A=$ stack area $=\pi^{*} r^{2}$ in $m$

\begin{tabular}{|c|c|c|c|c|c|c|c|c|c|}
\hline Configuration & $\begin{array}{c}\text { Stack Flow } \\
\text { cfm }\end{array}$ & $\begin{array}{c}\text { Stack Flow } \\
\mathrm{m3} / \mathrm{s}\end{array}$ & $\begin{array}{c}\text { Diameter } \\
\text { in }\end{array}$ & $\begin{array}{c}\text { Hydraulic } \\
\text { Diameter } \\
\mathrm{ft}\end{array}$ & $\begin{array}{c}\text { Hydraulic } \\
\text { Diameter } \\
\mathrm{m}\end{array}$ & $\begin{array}{c}\text { Area } \\
\mathrm{m} 2\end{array}$ & $\begin{array}{c}\mathrm{V} \\
\mathrm{ft} / \mathrm{min}\end{array}$ & $\begin{array}{c}\mathrm{V} \\
\mathrm{m} / \mathrm{s}\end{array}$ & $\mathrm{Re}$ \\
\hline High Flow (Actual) & 145,400 & 68.34 & 96 & 8.00 & 2.44 & 4.667 & 2894 & 14.6 & $2,275,629$ \\
\hline Low Flow (Actual) & 133,000 & 62.51 & 96 & 8.00 & 2.44 & 4.667 & 2647 & 13.4 & $2,081,559$ \\
\hline High - 1 fan (Proposed) & 48,467 & 22.78 & 96 & 8.00 & 2.44 & 4.667 & 965 & 4.9 & 758,543 \\
\hline Low - 1 fan (Proposed) & 44,333 & 20.84 & 96 & 8.00 & 2.44 & 4.667 & 882 & 4.5 & 693,853 \\
\hline Scale Model - 3 fan & 5,823 & 2.74 & 18 & 1.50 & 0.46 & 0.164 & 3297 & 16.7 & 486,052 \\
\hline Scale Model - 3 fan & 4,391 & 2.06 & 18 & 1.50 & 0.46 & 0.164 & 2486 & 12.6 & 366,521 \\
\hline Scale Model - 1 fan & 1,370 & 0.64 & 18 & 1.50 & 0.46 & 0.164 & 776 & 3.9 & 114,355 \\
\hline Scale Model - 1 fan & 1,413 & 0.66 & 18 & 1.50 & 0.46 & 0.164 & 800 & 4.0 & 117,945 \\
\hline Scale Model - 1 fan & 1,100 & 0.52 & 18 & 1.50 & 0.46 & 0.164 & 623 & 3.2 & 91,818 \\
\hline
\end{tabular}

Flow range using scale model:

\begin{tabular}{|c|c|c|c|c|c|c|c|c|c|c|c|}
\hline \multirow[t]{2}{*}{ Test } & \multirow[t]{2}{*}{$\mathrm{Hz}$} & \multirow{2}{*}{$\begin{array}{l}\text { Average } \\
\text { Velocity, fpm }\end{array}$} & \multirow{2}{*}{$\begin{array}{l}\text { hydraulic } \\
\text { diameter, ft }\end{array}$} & \multirow{2}{*}{$\begin{array}{l}\text { Velocity } x \text { diam } \\
\mathrm{ft} 2 / \mathrm{min}\end{array}$} & \multirow[t]{2}{*}{ Six Times } & \multirow[t]{2}{*}{ One-Sixth } & \multirow{2}{*}{$\begin{array}{l}\text { stack diam } \\
\mathrm{ft}\end{array}$} & \multicolumn{2}{|c|}{ Stack Vel fpm } & \multicolumn{2}{|c|}{ Stack flow cfm } \\
\hline & & & & & & & & High & Low & High & Low \\
\hline VT-LOW1 & 30 & 622 & 1.5 & 933 & 5598 & 155.5 & 8 & 699.8 & 19.4 & 35155 & 977 \\
\hline VT-1 & 37.1 & 786 & 1.5 & 1179 & 7074 & 196.5 & 8 & 884.3 & 24.6 & 44425 & 1234 \\
\hline VT-3 & 37.1 & 804 & 1.5 & 1206 & 7236 & 201 & 8 & 904.5 & 25.1 & 45442 & 1262 \\
\hline VT-2 & 37.1 & 755 & 1.5 & 1132.5 & 6795 & 188.75 & 8 & 849.4 & 23.6 & 42673 & 1185 \\
\hline VT-4 & 37.1 & 756 & 1.5 & 1134 & 6804 & 189 & 8 & 850.5 & 23.6 & 42729 & 1187 \\
\hline & & & & & & area $(\mathrm{ft} 2)=$ & 50.24 & & & & \\
\hline
\end{tabular}





\section{Appendix $\mathrm{H}$}

Computational Fluid Dynamics Model Details 



\section{Computational Fluid Dynamics Model Details}

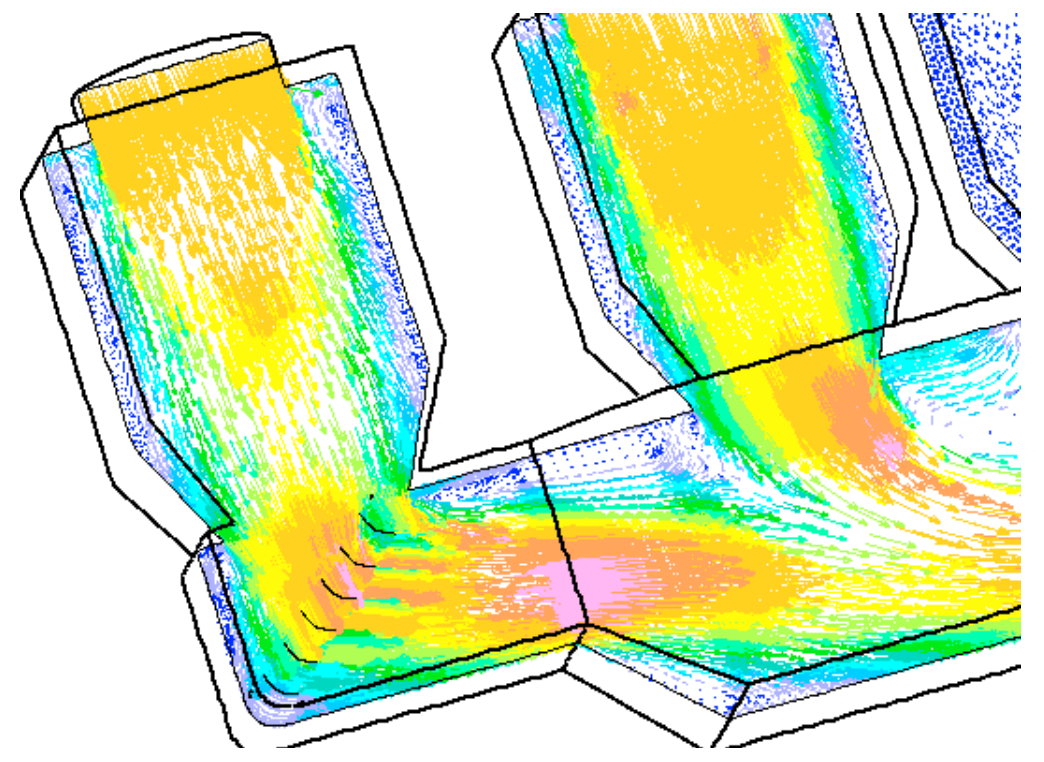

Figure H.1. Detail of flow vectors entering the main duct via fans \#1 and \#2 (for configuration of fans 1 $\& 2$ farthest from the stack operational).

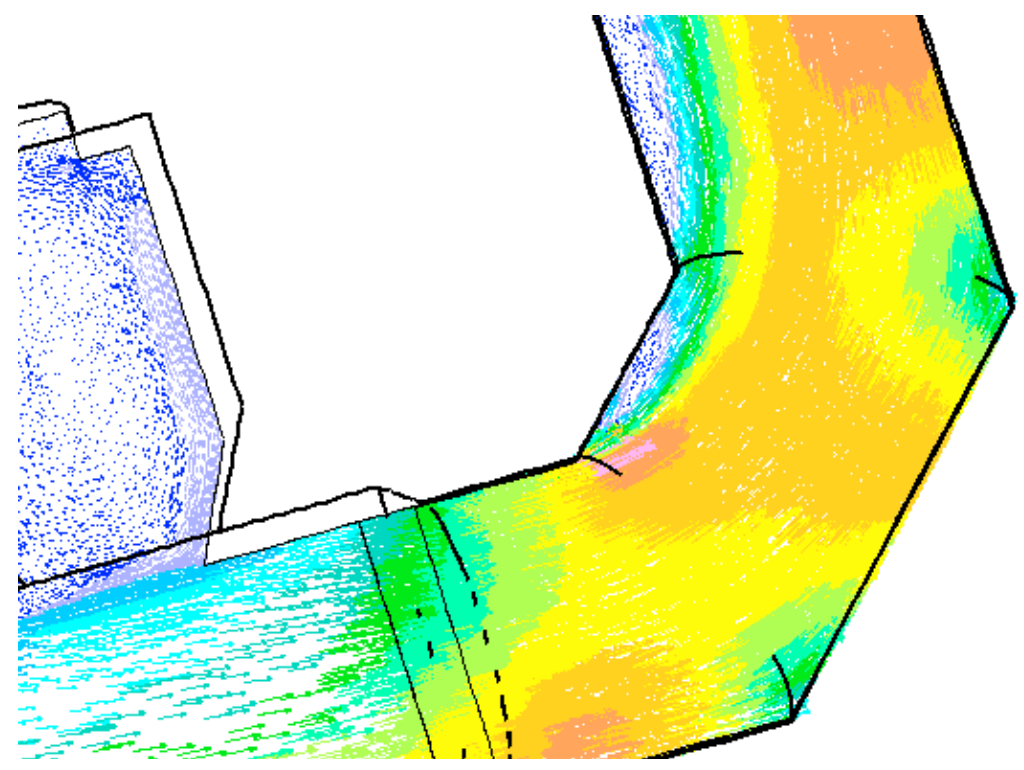

Figure H.2. Detail of flow vectors in the $90^{\circ}$ bend in the horizontal duct (for configuration of fans $1 \& 2$ farthest from the stack operational). 


\section{Results: Operation of Fans 1 and 2}

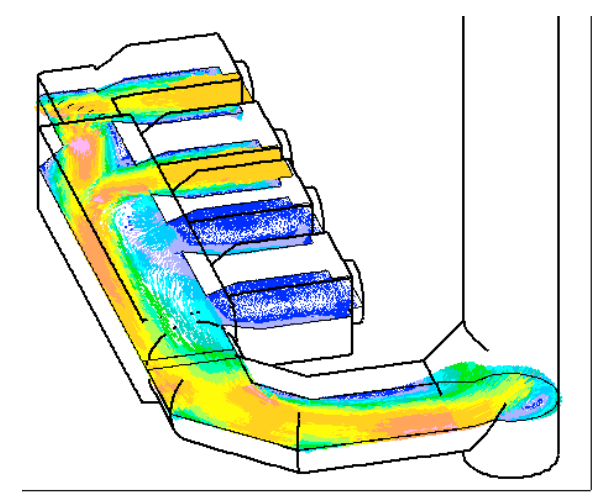

a)

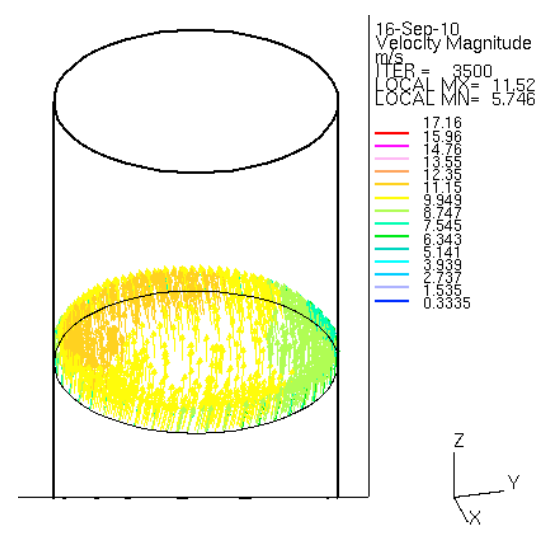

c)

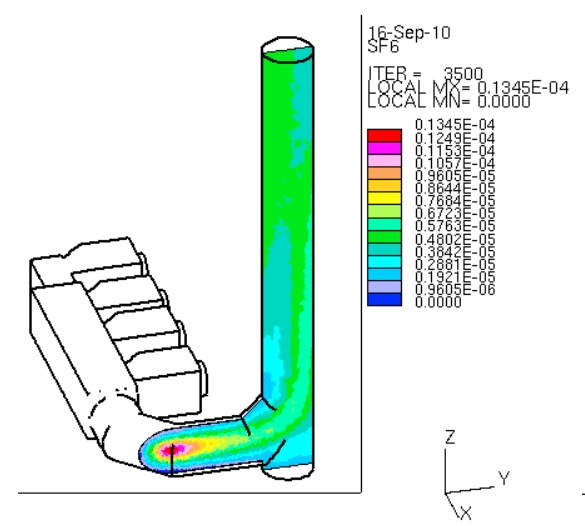

e)

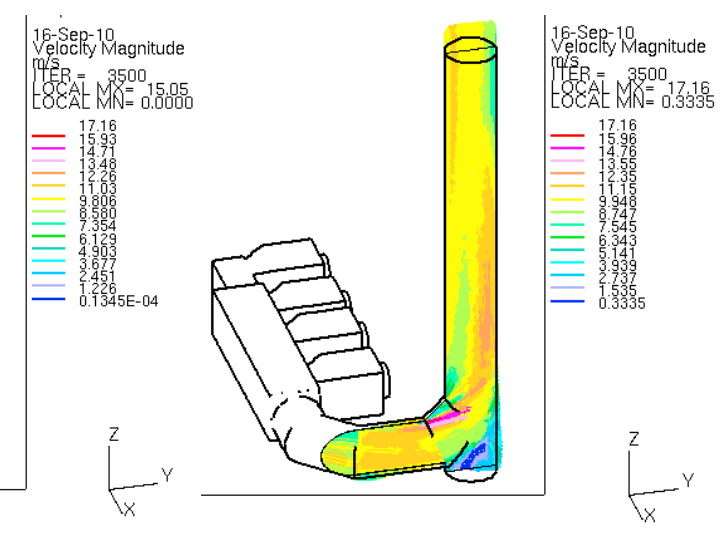

b)

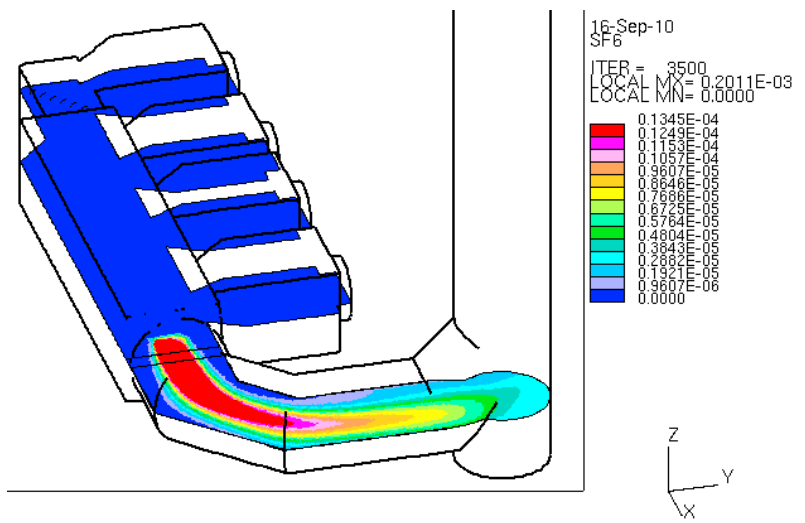

d)

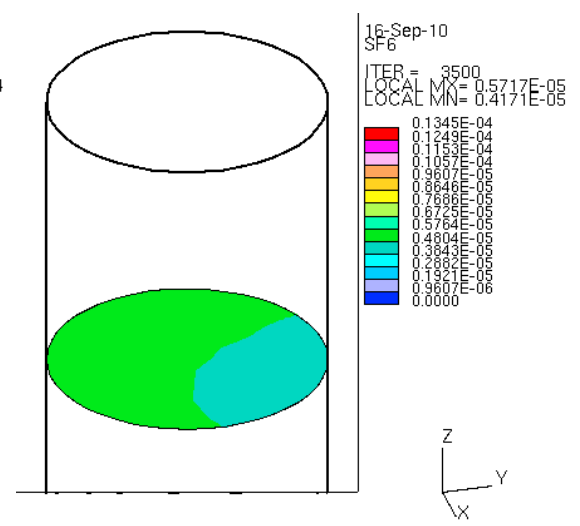

f)

Figure H.3. Simulated flow velocity vectors and tracer gas concentrations for the case with fans 1 and 2 in operation: a) flow velocity in fans and lower ductwork, b) flow velocity in stack, c) velocity distribution in the stack at the elevation of the sampling system, d) tracer gas concentrations in the lower ductwork; tracer gas injected downstream of the fan nearest the stack, e) tracer gas concentrations in the stack, f) tracer gas distribution in the stack at the elevation of the sampling system. 


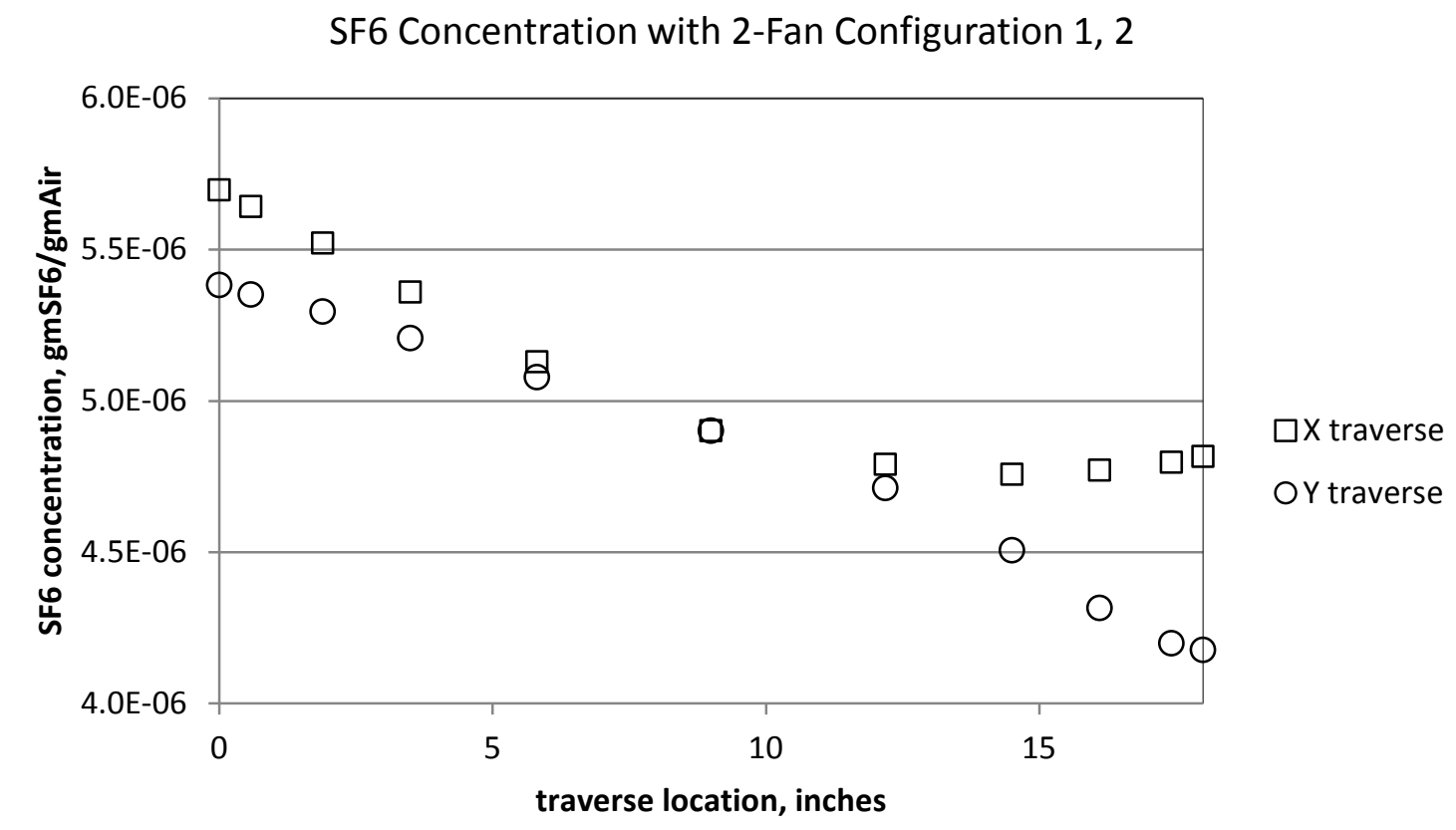

a)

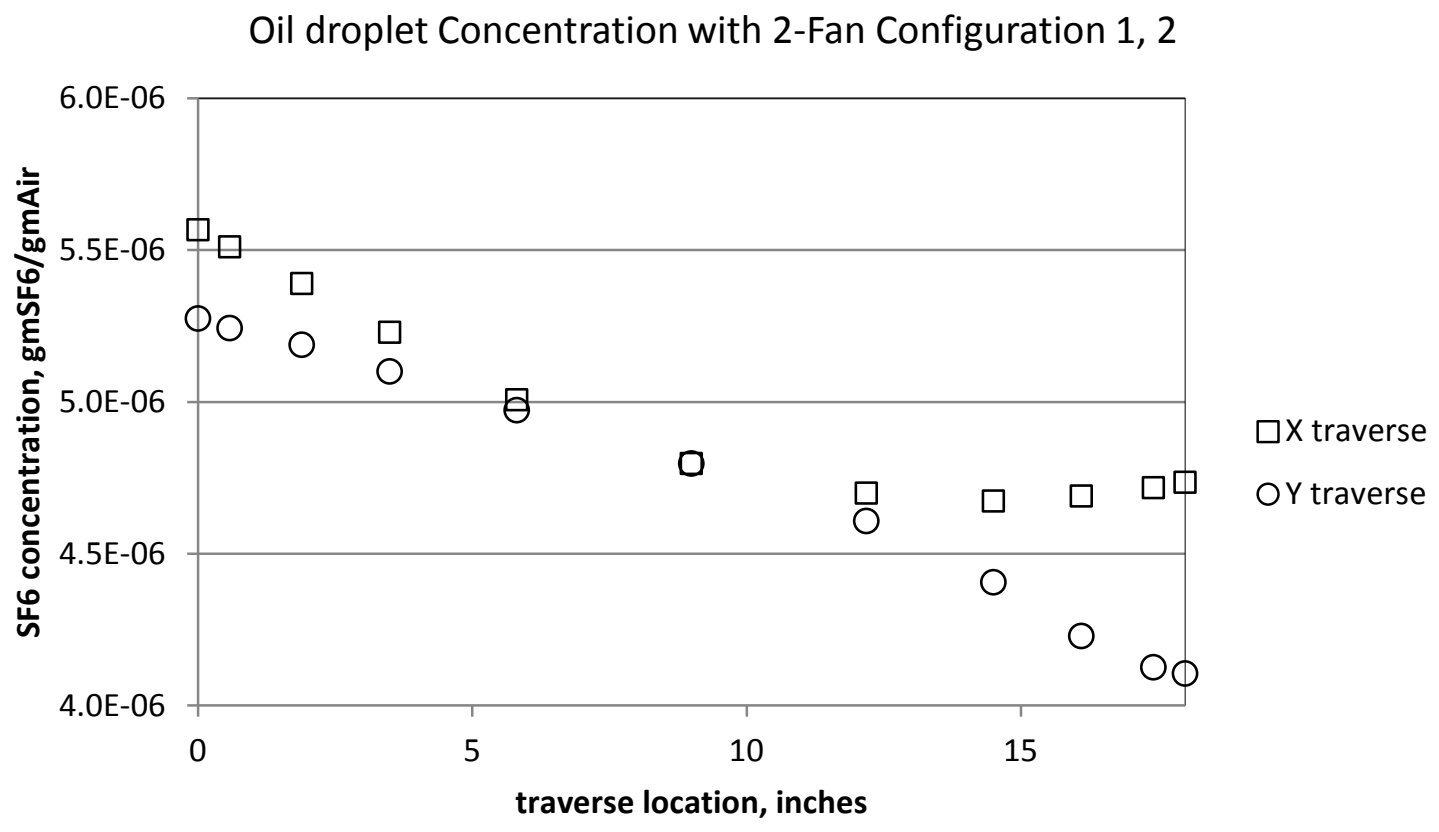

b)

Figure H.4. Simulated results for the 2-fan configuration 1,2: Concentrations of a) SF6 tracer gas, and b) oil droplets at the elevation of the sampling system. 
Flow Velocity with 2-Fan Configuration 1, 2

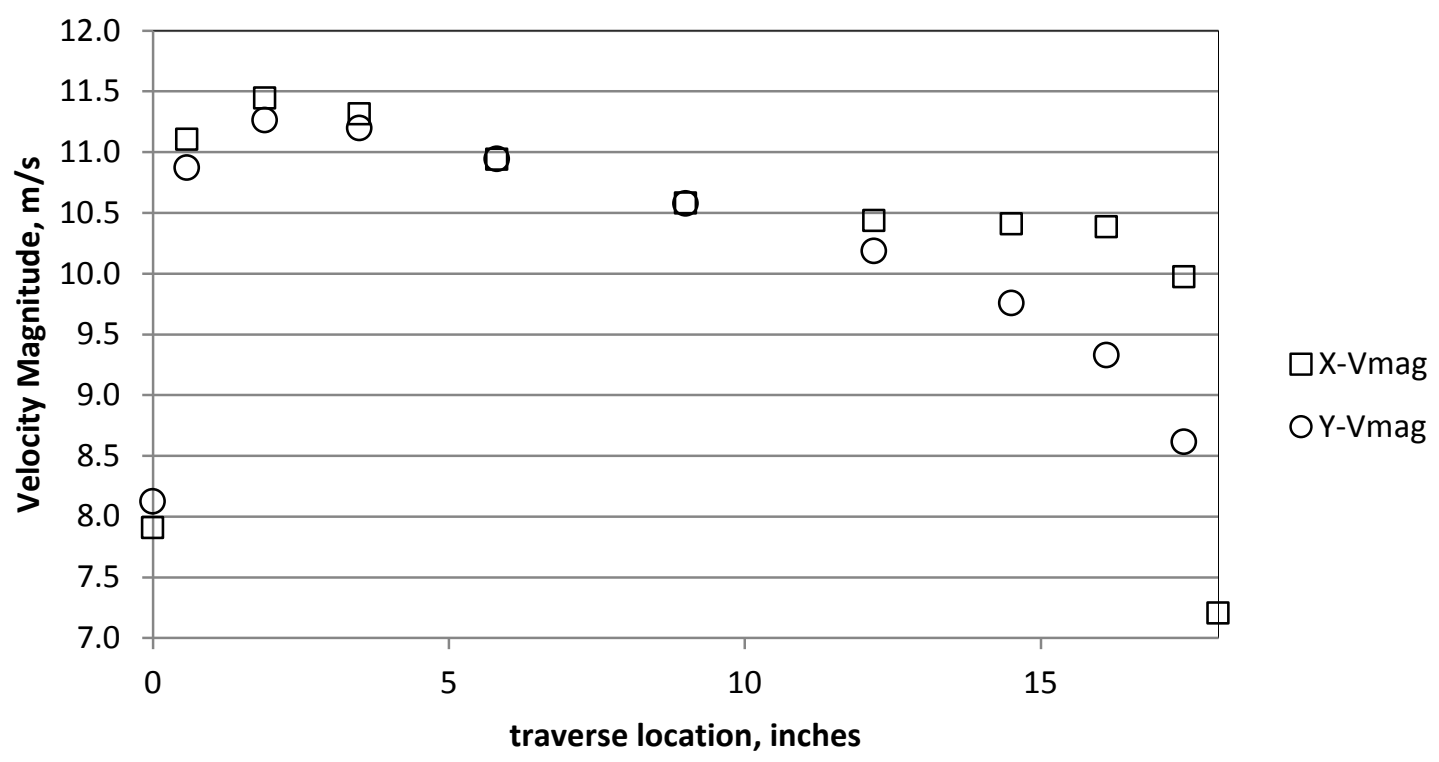

Figure H.5. Simulated results for the 2-fan configuration 1, 2: Flow velocity distribution at the elevation of the sampling system.

Flow Angle with 2-Fan Configuration 1, 2

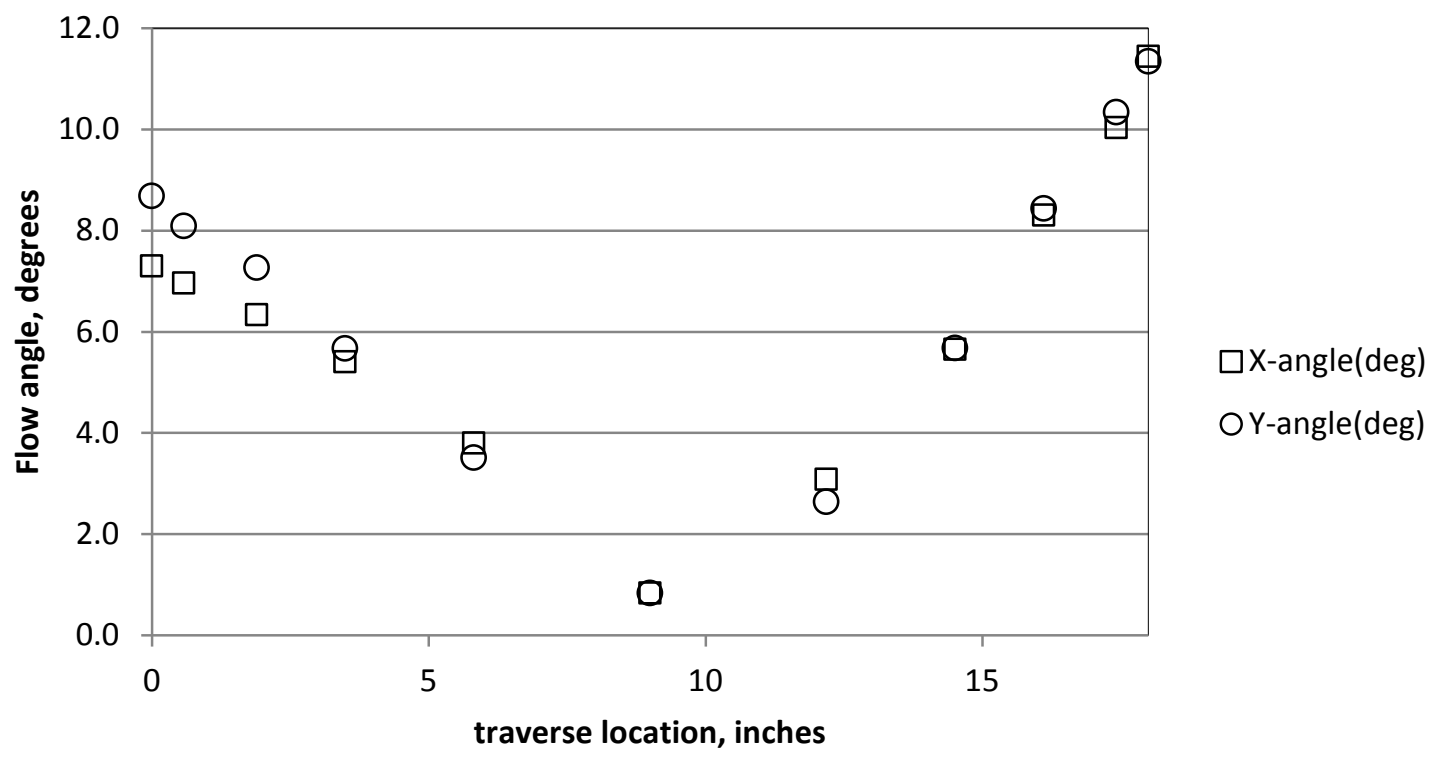

Figure H.6. Simulated results for the 2-fan configuration 1, 2: Cyclonic flow angle at the elevation of the sampling system. 


\section{Results: Operation of Fans 1 and 3}

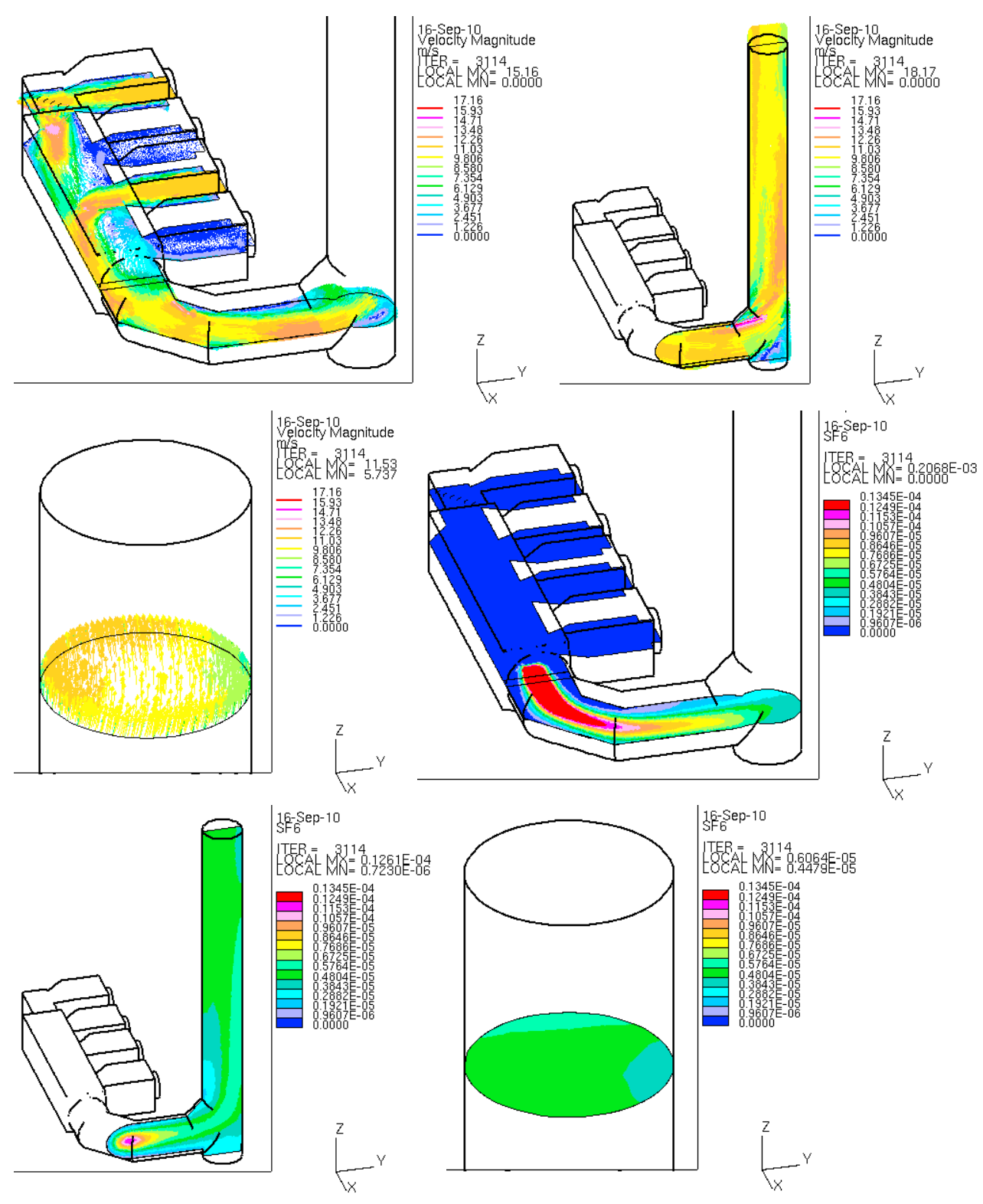

Figure H.7. Simulated flow velocity vectors and tracer gas concentrations for the case with fans 1 and 3 in operation: a) flow velocity in fans and lower ductwork, b) flow velocity in stack, c) velocity distribution in the stack at the elevation of the sampling system, d) tracer gas concentrations in the lower ductwork; tracer gas injected downstream of the fan nearest the stack, e) tracer gas concentrations in the stack, f) tracer gas distribution in the stack at the elevation of the sampling system. 


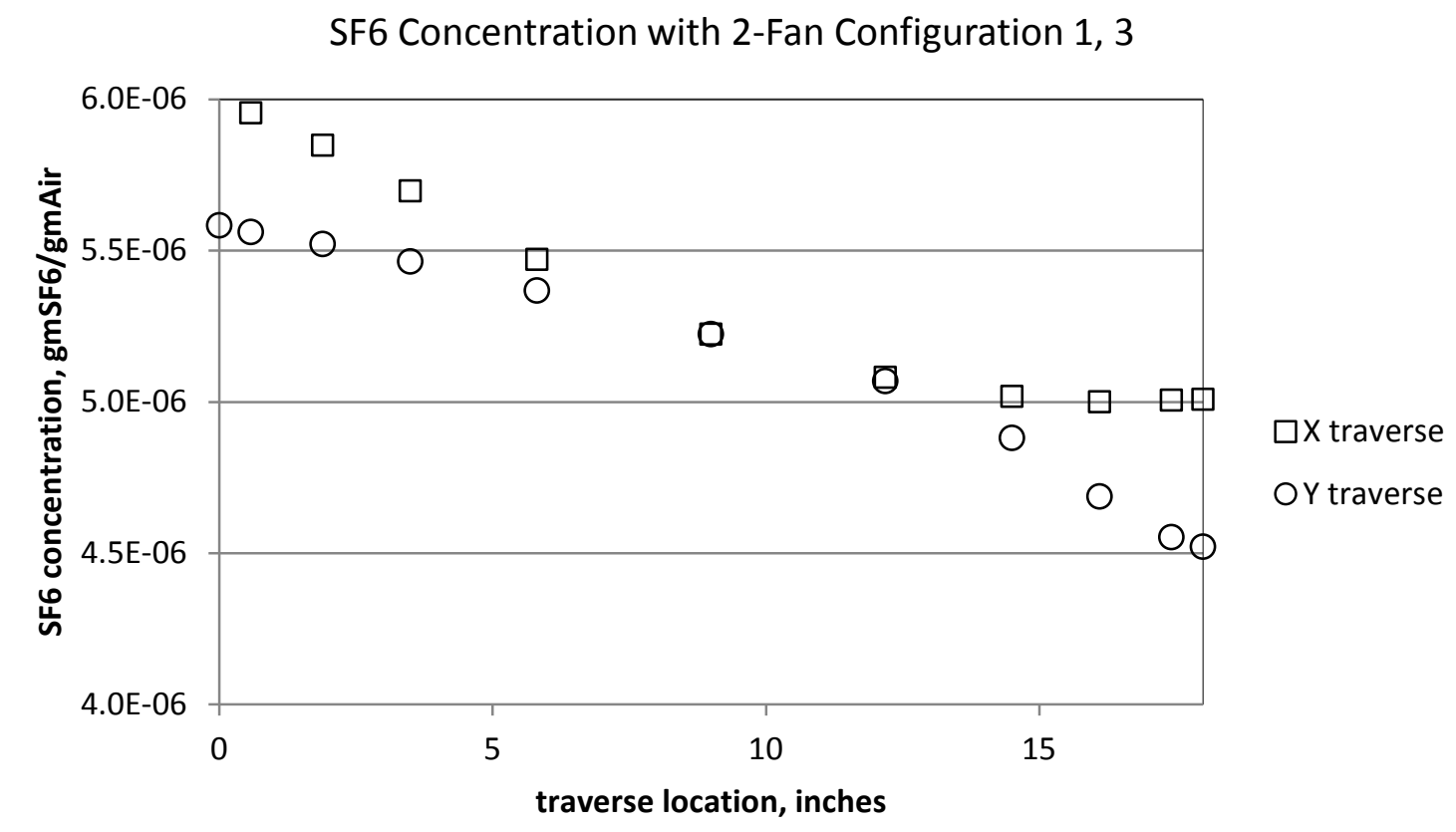

a)

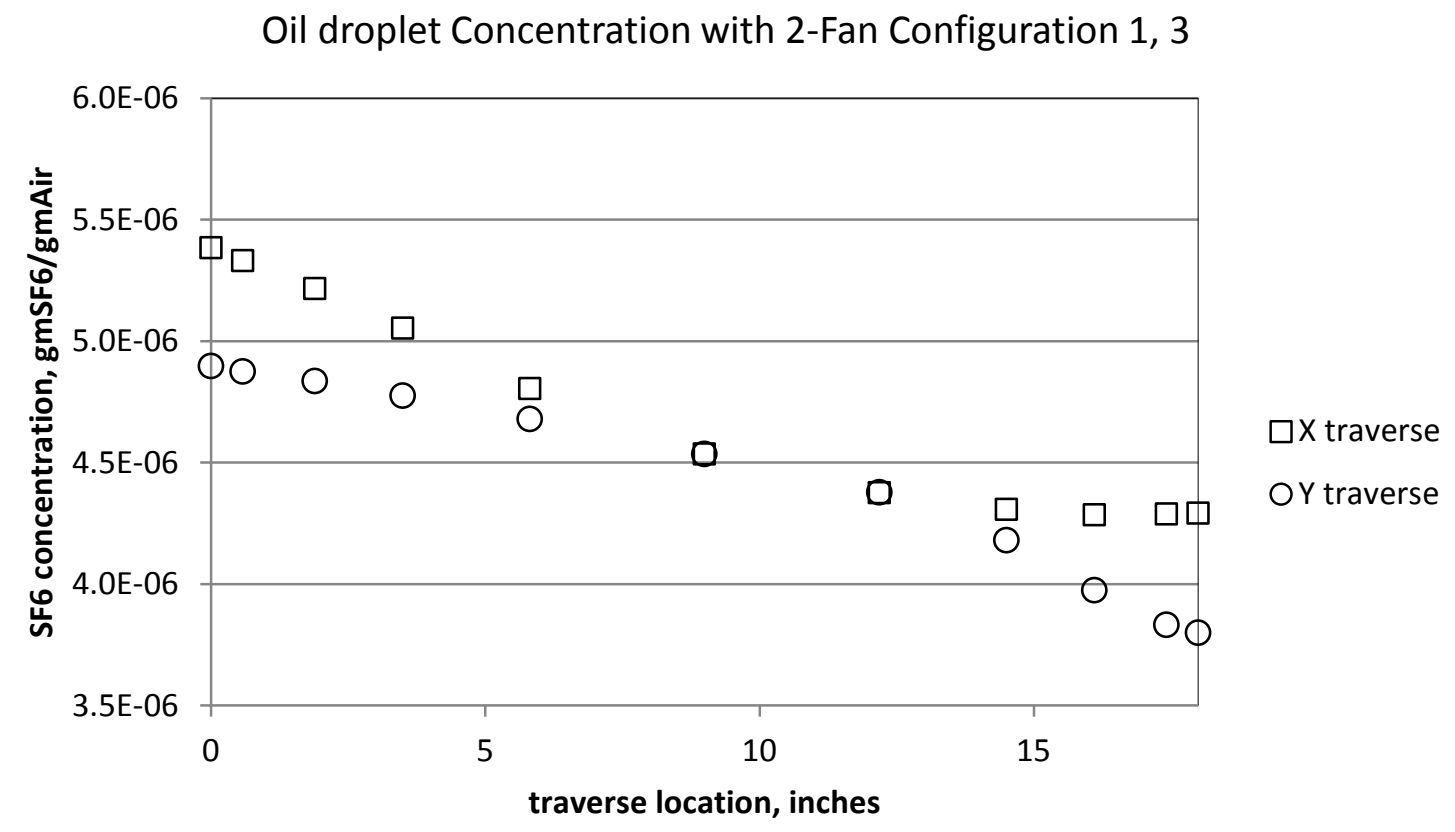

b)

Figure H.8. Simulated results for the 2-fan configuration 1, 3: Concentrations of a) SF6 tracer gas, and b) oil droplets at the elevation of the sampling system. 


\section{Flow Velocity with 2-Fan Configuration 1, 3}

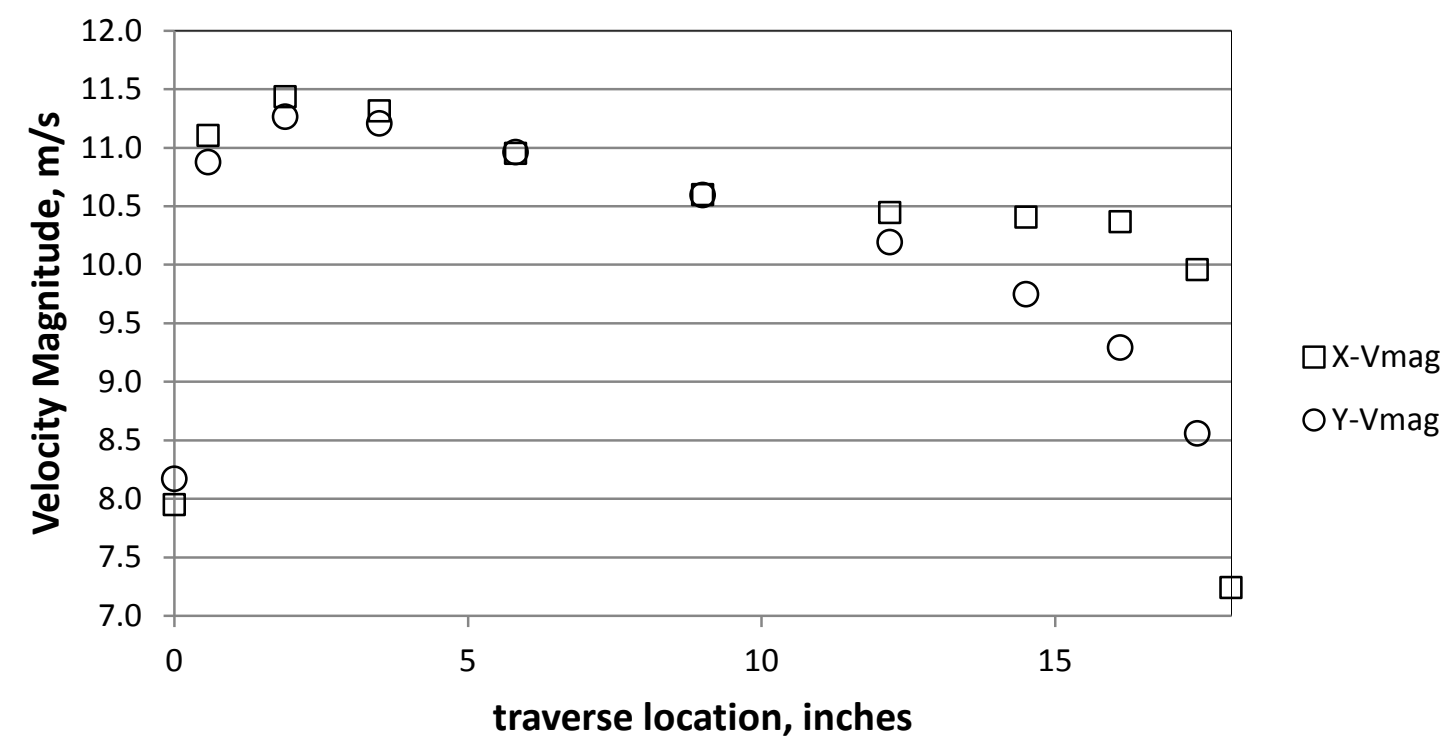

Figure H.9. Simulated results for the 2-fan configuration 1, 3: Flow velocity distribution at the elevation of the sampling system.

Flow Angle with 2-Fan Configuration 1, 3

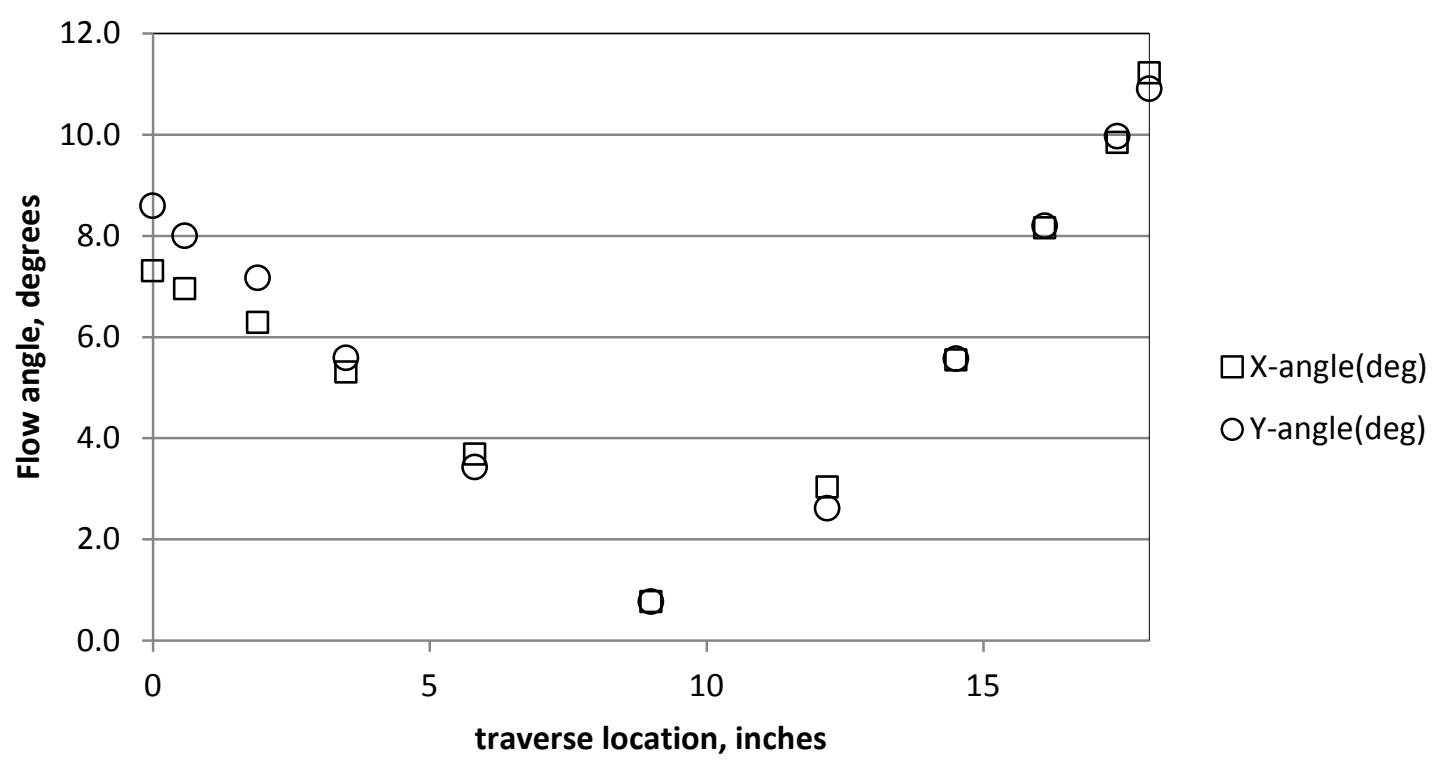

Figure H.10. Simulated results for the 2-fan configuration 1, 3: Cyclonic flow angle at the elevation of the sampling system. 


\section{Results: Operation of Fans 1 and 4}

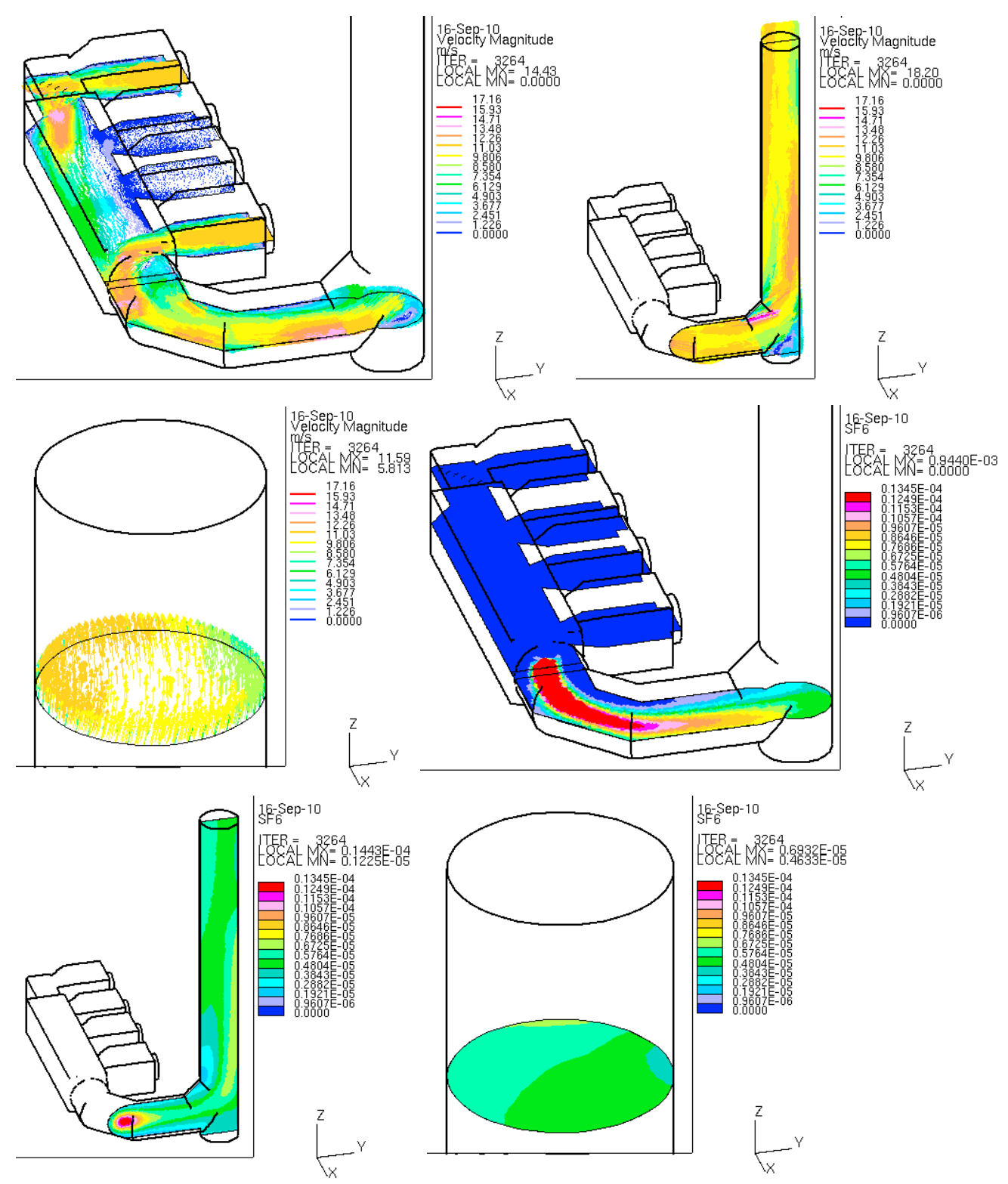

Figure H.11. Simulated flow velocity vectors and tracer gas concentrations for the case with fans 1 and 4 in operation: a) flow velocity in fans and lower ductwork, b) flow velocity in stack, c) velocity distribution in the stack at the elevation of the sampling system, d) tracer gas concentrations in the lower ductwork; tracer gas injected downstream of the fan nearest the stack, e) tracer gas concentrations in the stack, f) tracer gas distribution in the stack at the elevation of the sampling system. 


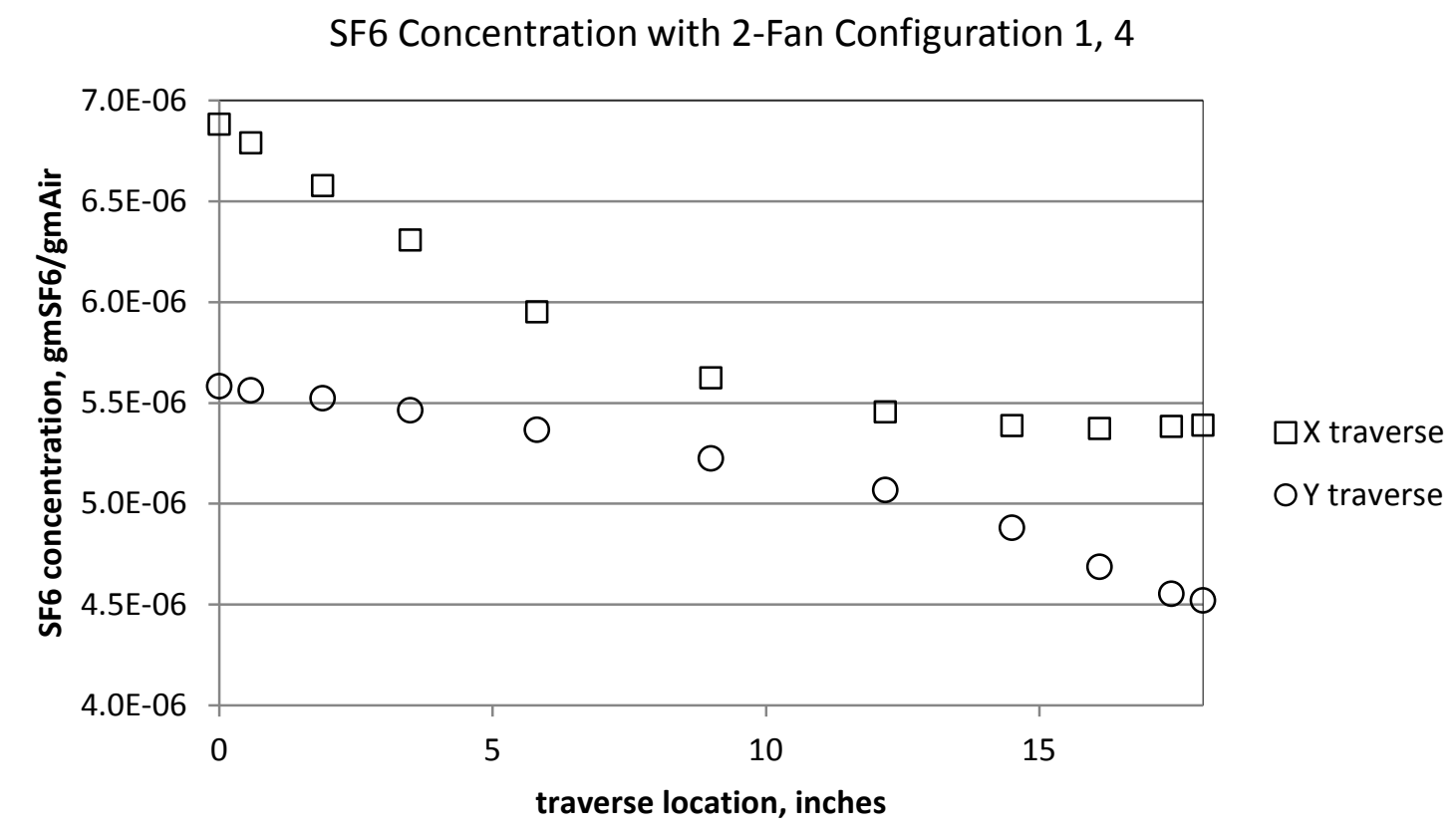

a)

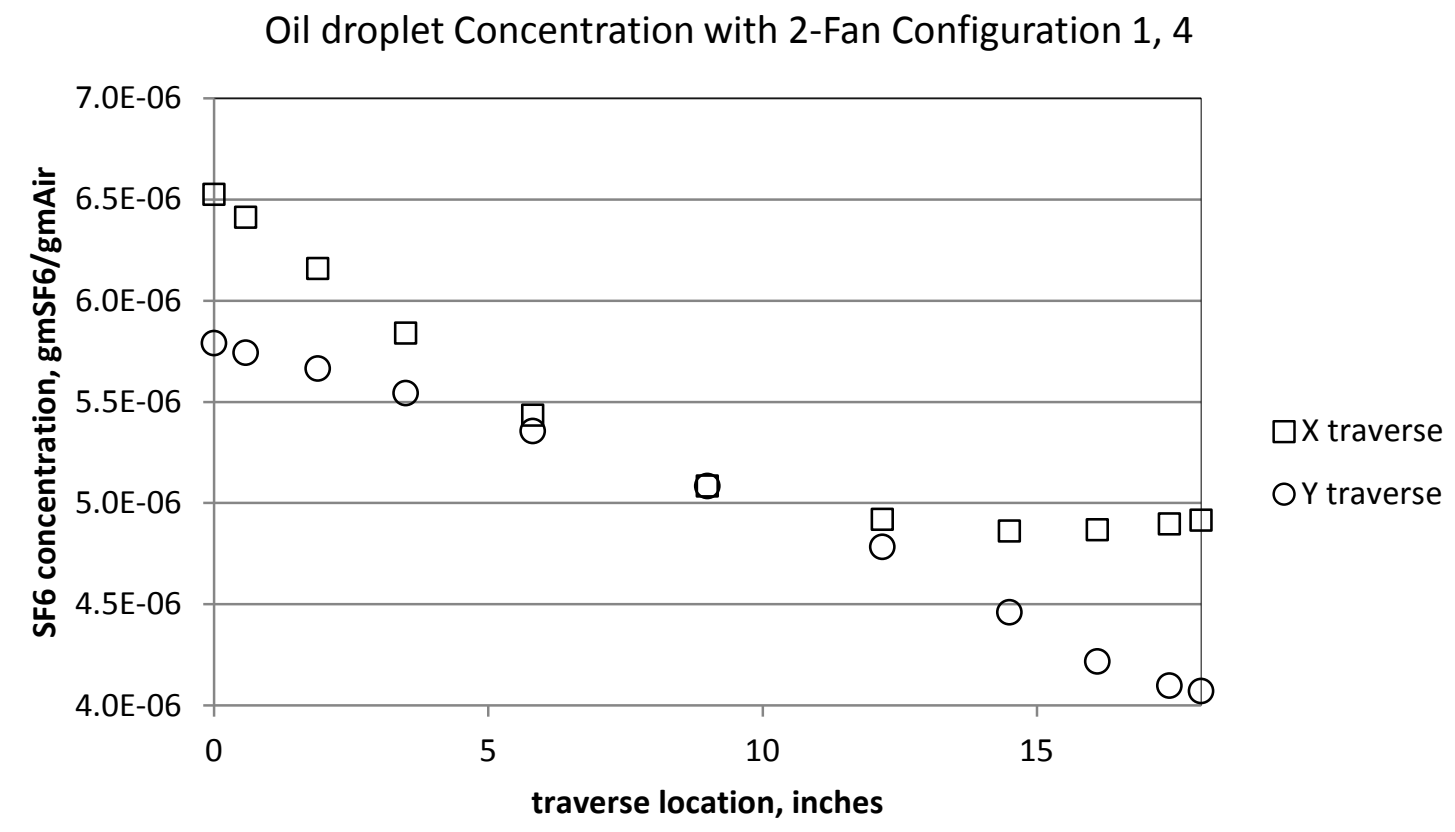

b)

Figure H.12. Simulated results for the 2-fan configuration 1, 4: Concentrations of a) SF6 tracer gas, and b) oil droplets at the elevation of the sampling system. 
Flow Velocity with 2-Fan Configuration 1, 4

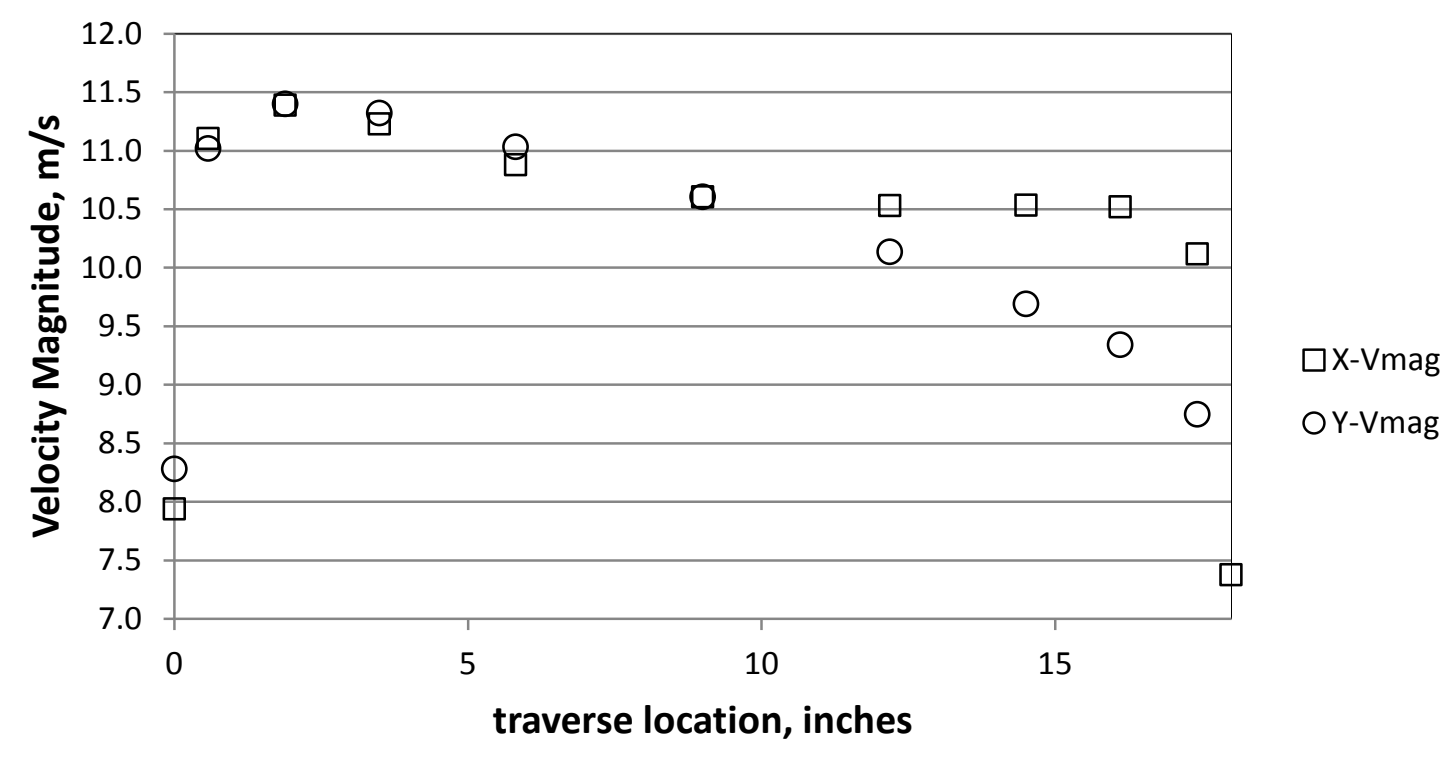

Figure H.13. Simulated results for the 2-fan configuration 1, 4: Flow velocity distribution at the elevation of the sampling system.

Flow Angle with 2-Fan Configuration 1, 4

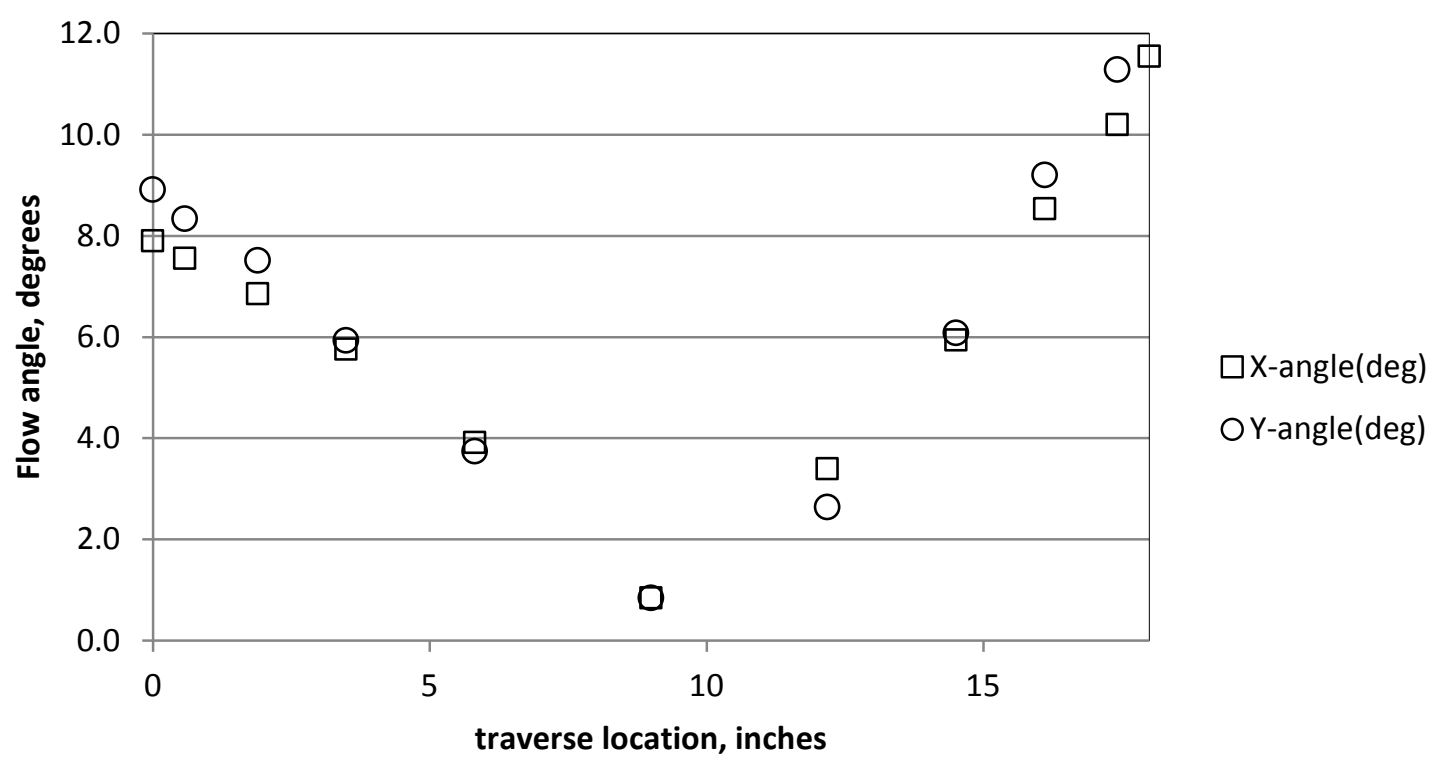

Figure H.14. Simulated results for the 2-fan configuration 1, 4: Cyclonic flow angle at the elevation of the sampling system. 


\section{Results: Operation of Fans 2 and 3}
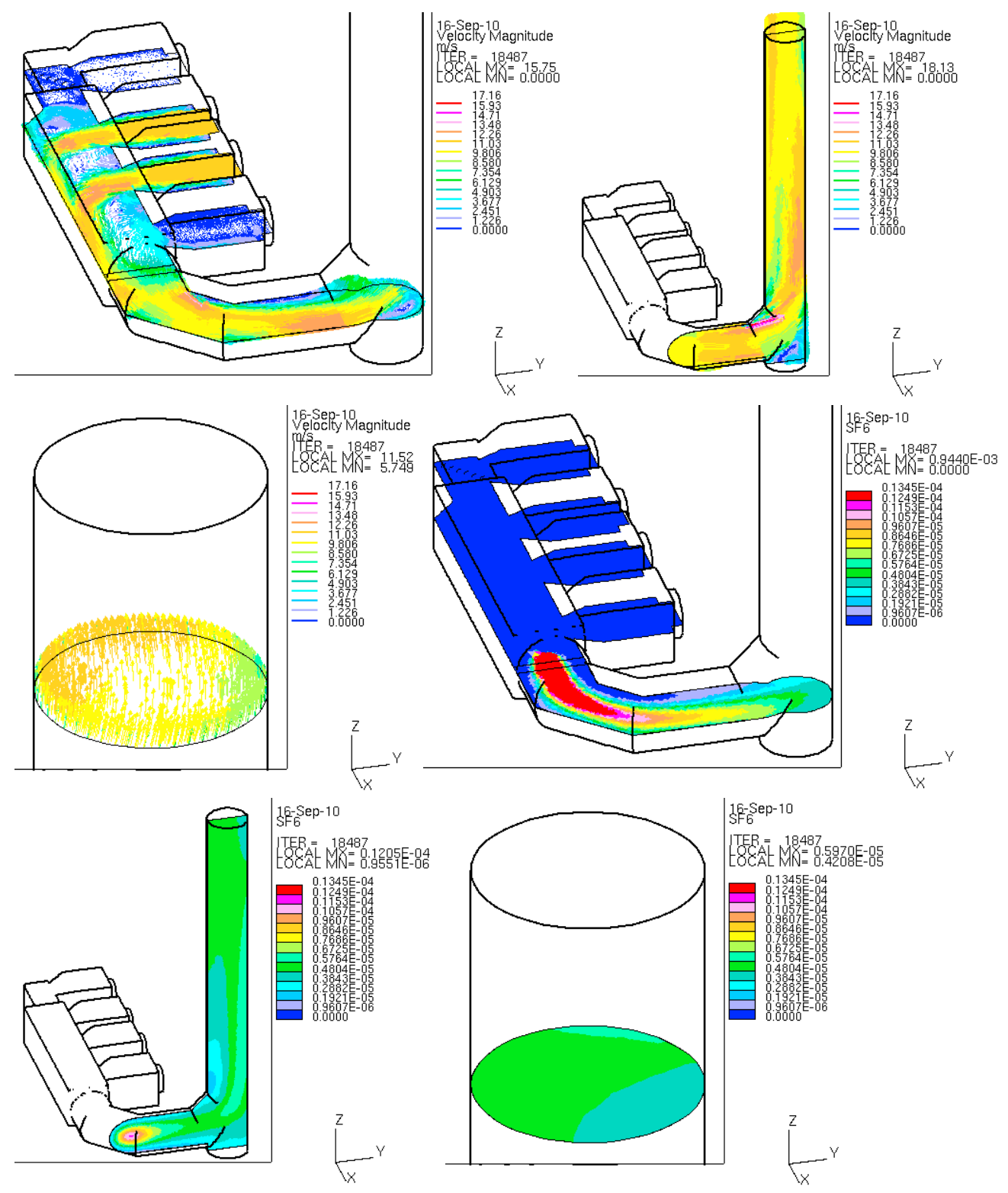

Figure H.15. Simulated flow velocity vectors and tracer gas concentrations for the case with fans 2 and 3 in operation: a) flow velocity in fans and lower ductwork, b) flow velocity in stack, c) velocity distribution in the stack at the elevation of the sampling system, d) tracer gas concentrations in the lower ductwork; tracer gas injected downstream of the fan nearest the stack, e) tracer gas concentrations in the stack, f) tracer gas distribution in the stack at the elevation of the sampling system. 


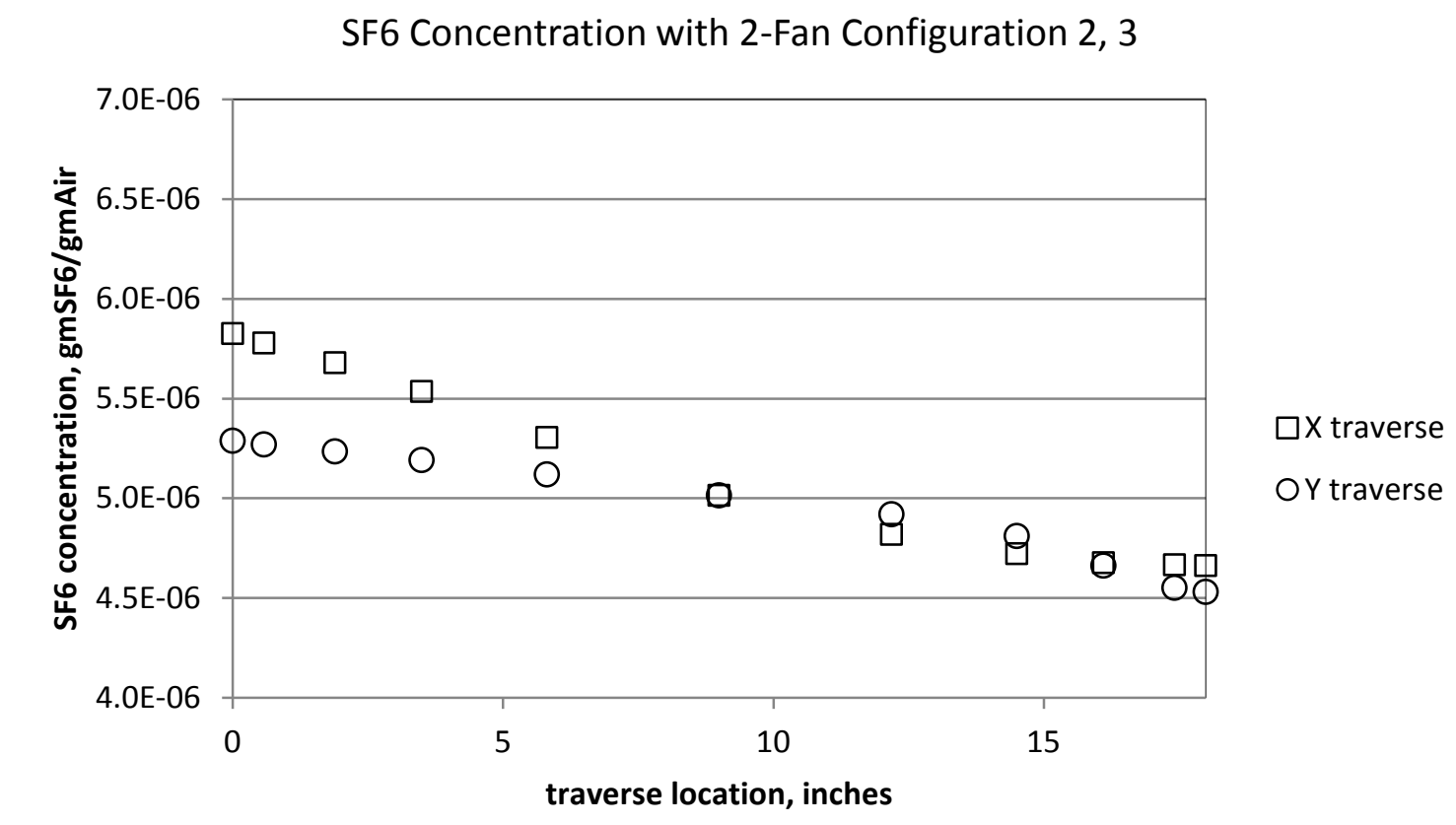

a)

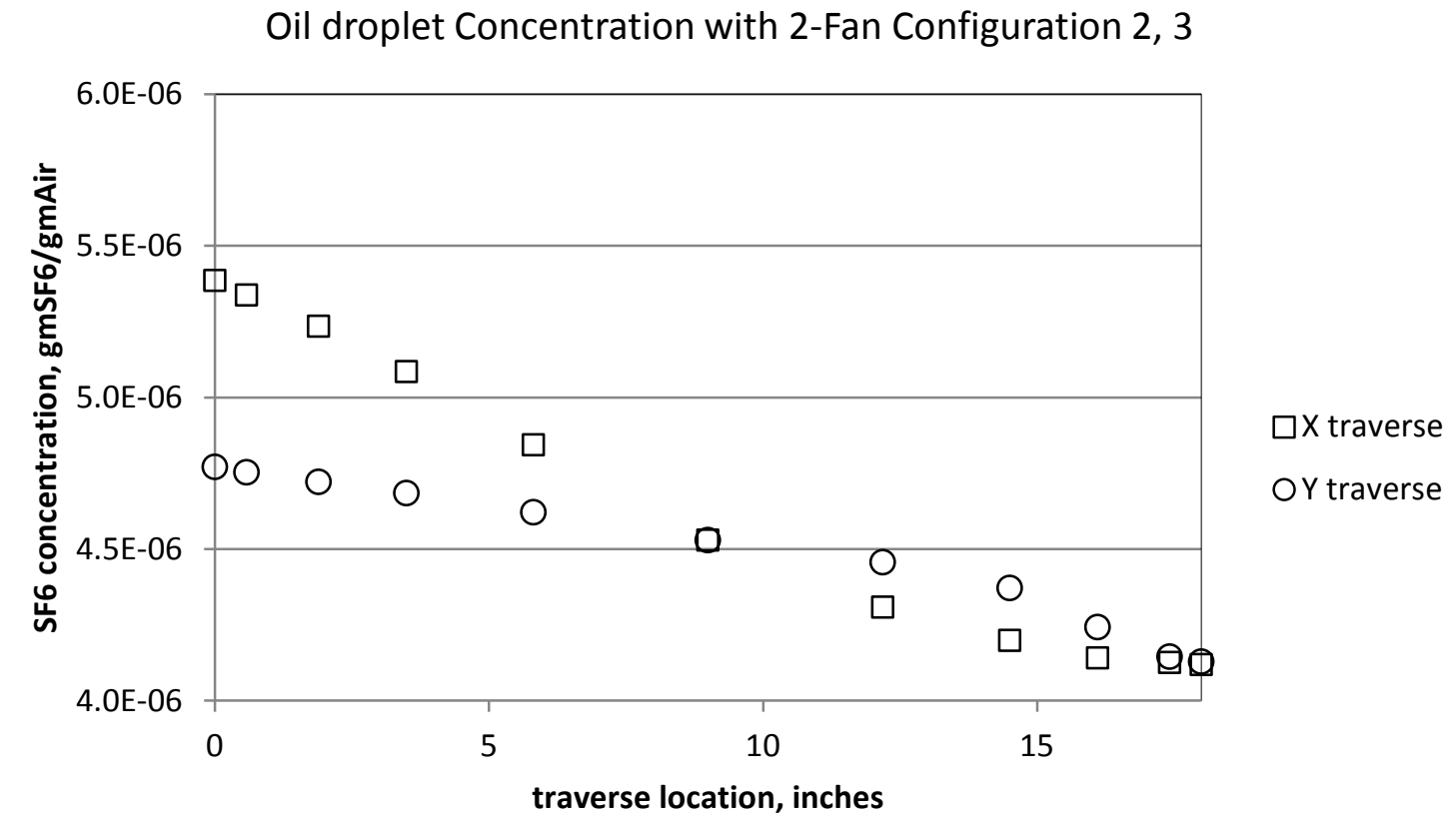

b)

Figure H.16. Simulated results for the 2-fan configuration 2, 3: Concentrations of a) SF6 tracer gas, and b) oil droplets at the elevation of the sampling system. 


\section{Flow Velocity with 2-Fan Configuration 2, 3}

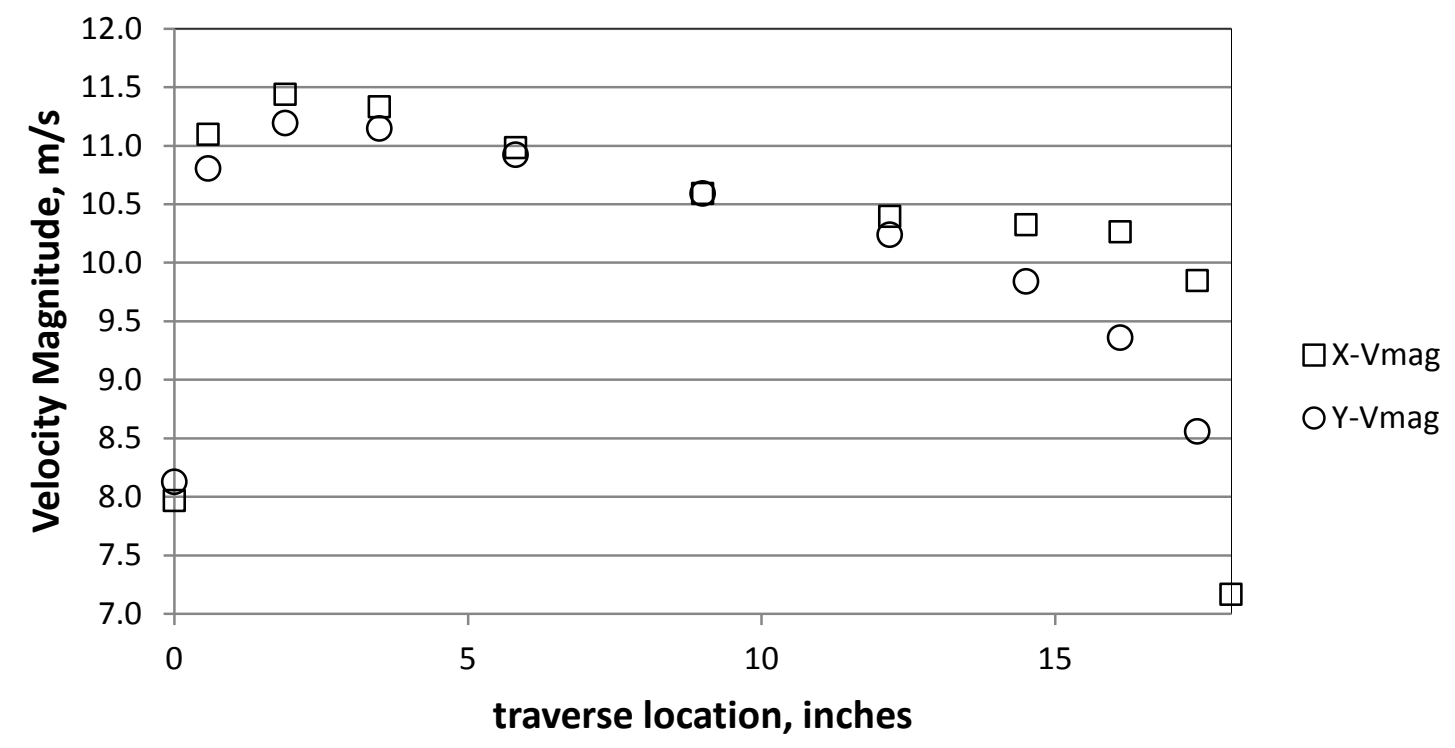

Figure H.17. Simulated results for the 2-fan configuration 2, 3: Flow velocity distribution at the elevation of the sampling system.

Flow Angle with 2-Fan Configuration 2, 3

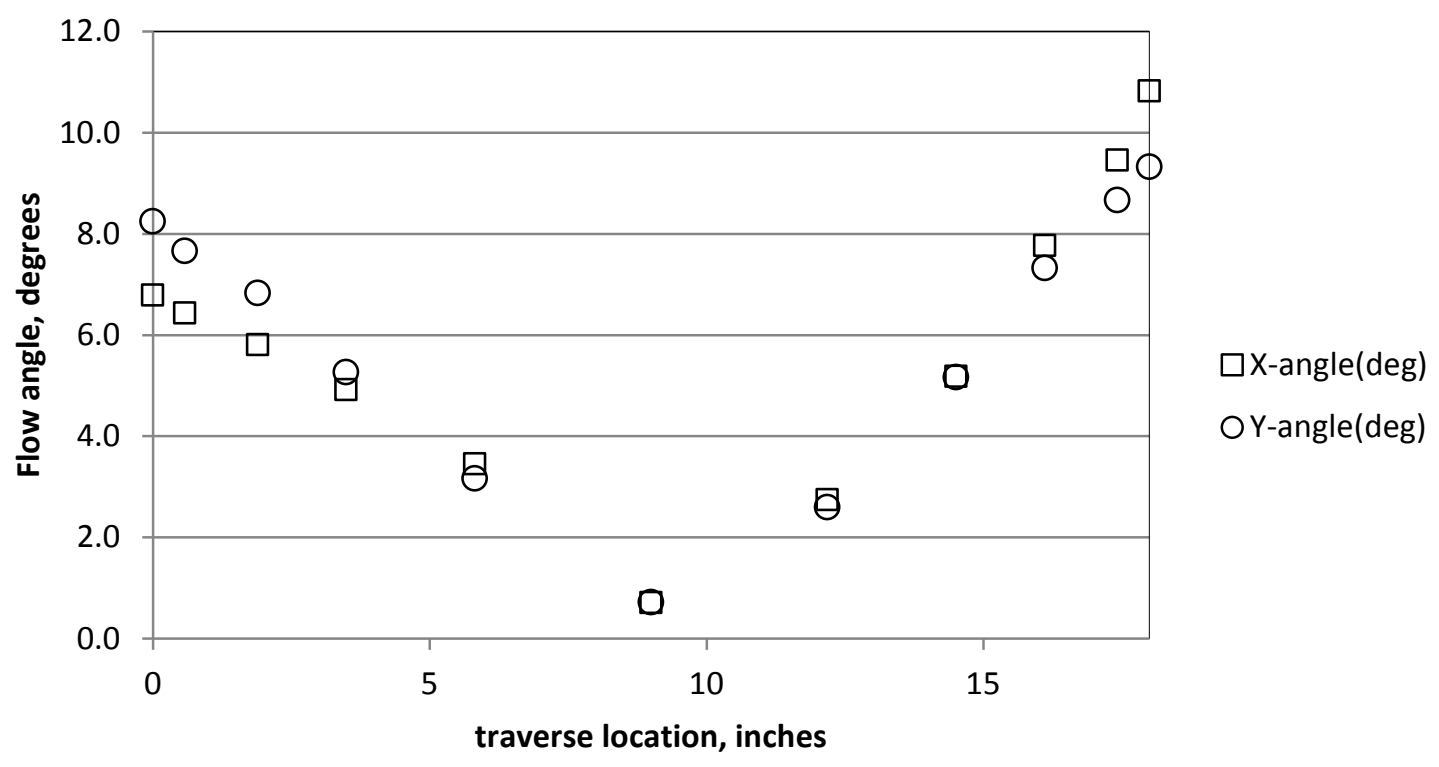

Figure H.18. Simulated results for the 2-fan configuration 2, 3: Cyclonic flow angle at the elevation of the sampling system. 


\section{Results: Operation of Fans 2 and 4}
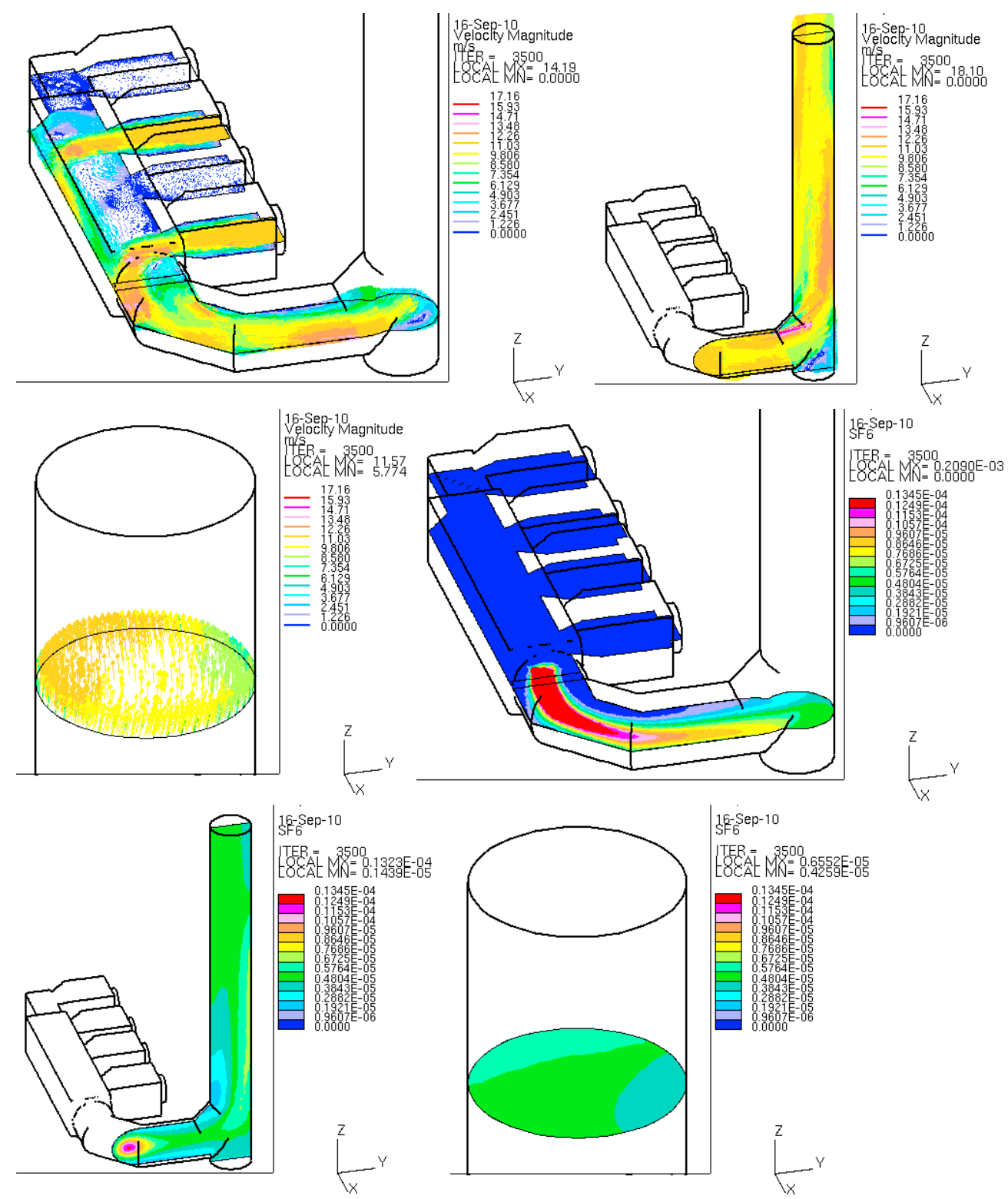

Figure H.19. Simulated flow velocity vectors and tracer gas concentrations for the case with fans 2 and 4 in operation: a) flow velocity in fans and lower ductwork, b) flow velocity in stack, c) velocity distribution in the stack at the elevation of the sampling system, d) tracer gas concentrations in the lower ductwork; tracer gas injected downstream of the fan nearest the stack, e) tracer gas concentrations in the stack, f) tracer gas distribution in the stack at the elevation of the sampling system. 


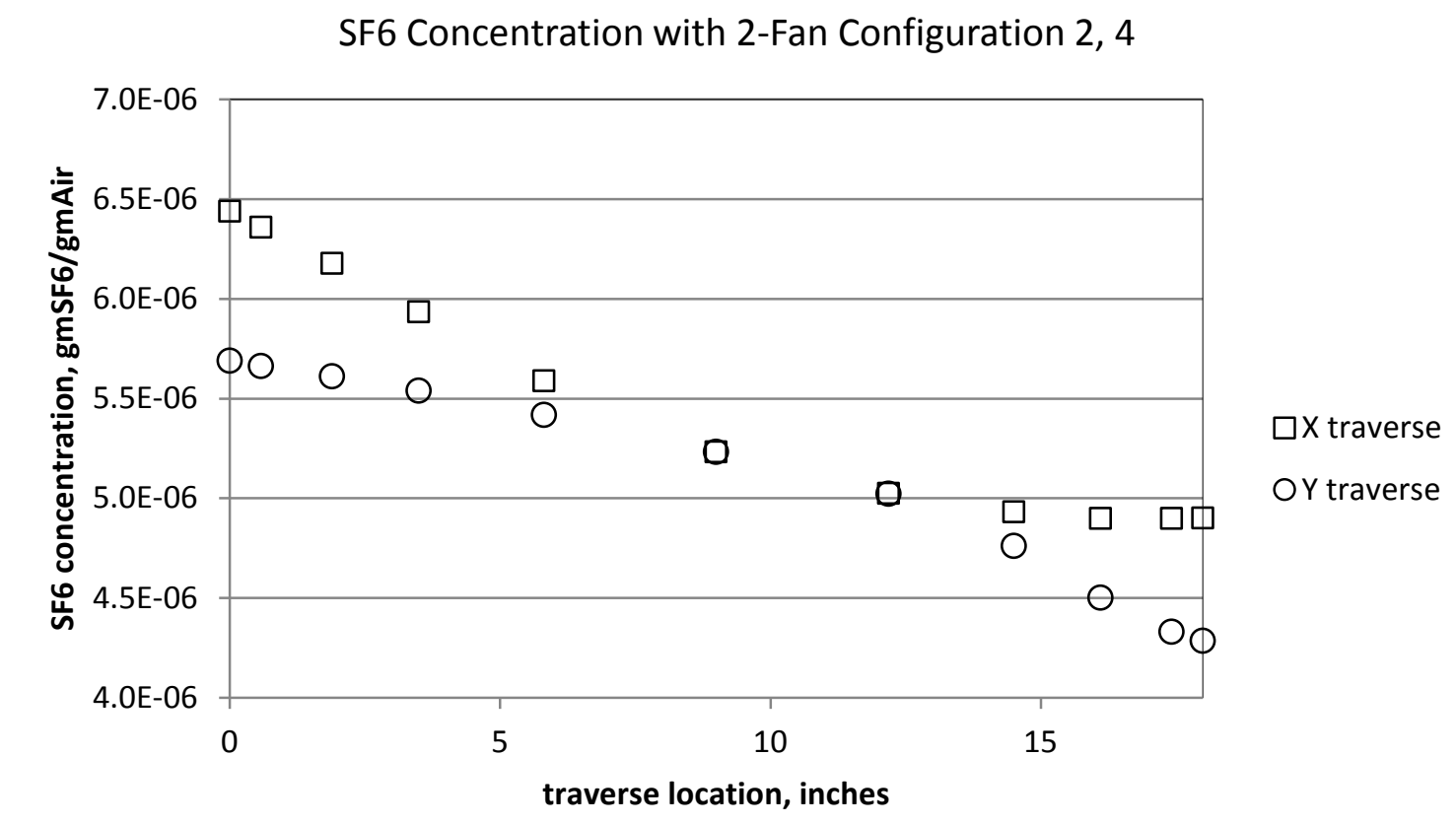

a)

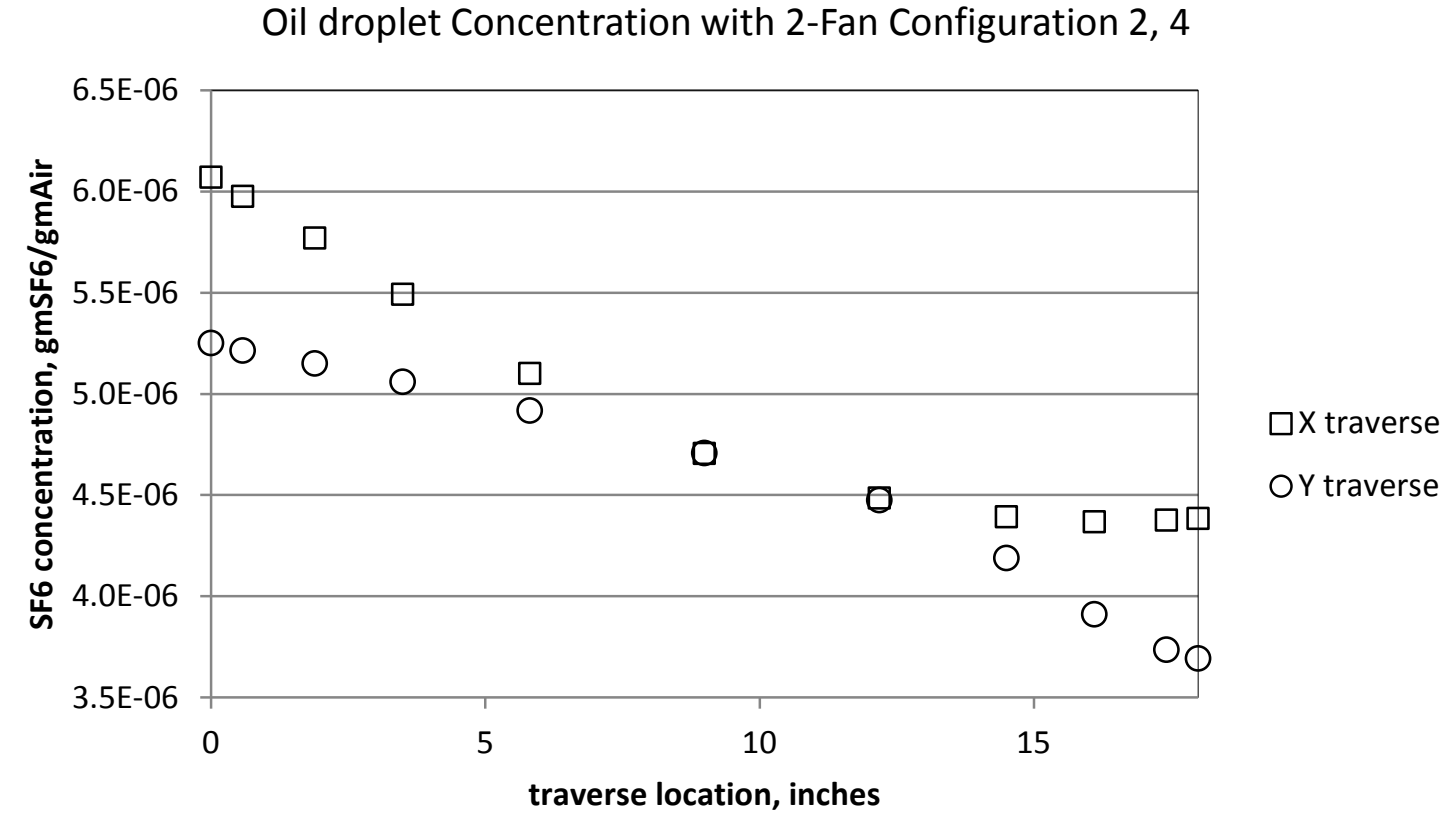

b)

Figure H.20. Simulated results for the 2-fan configuration 2, 4: Concentrations of a) SF6 tracer gas, and b) oil droplets at the elevation of the sampling system. 
Flow Velocity with 2-Fan Configuration 2, 4

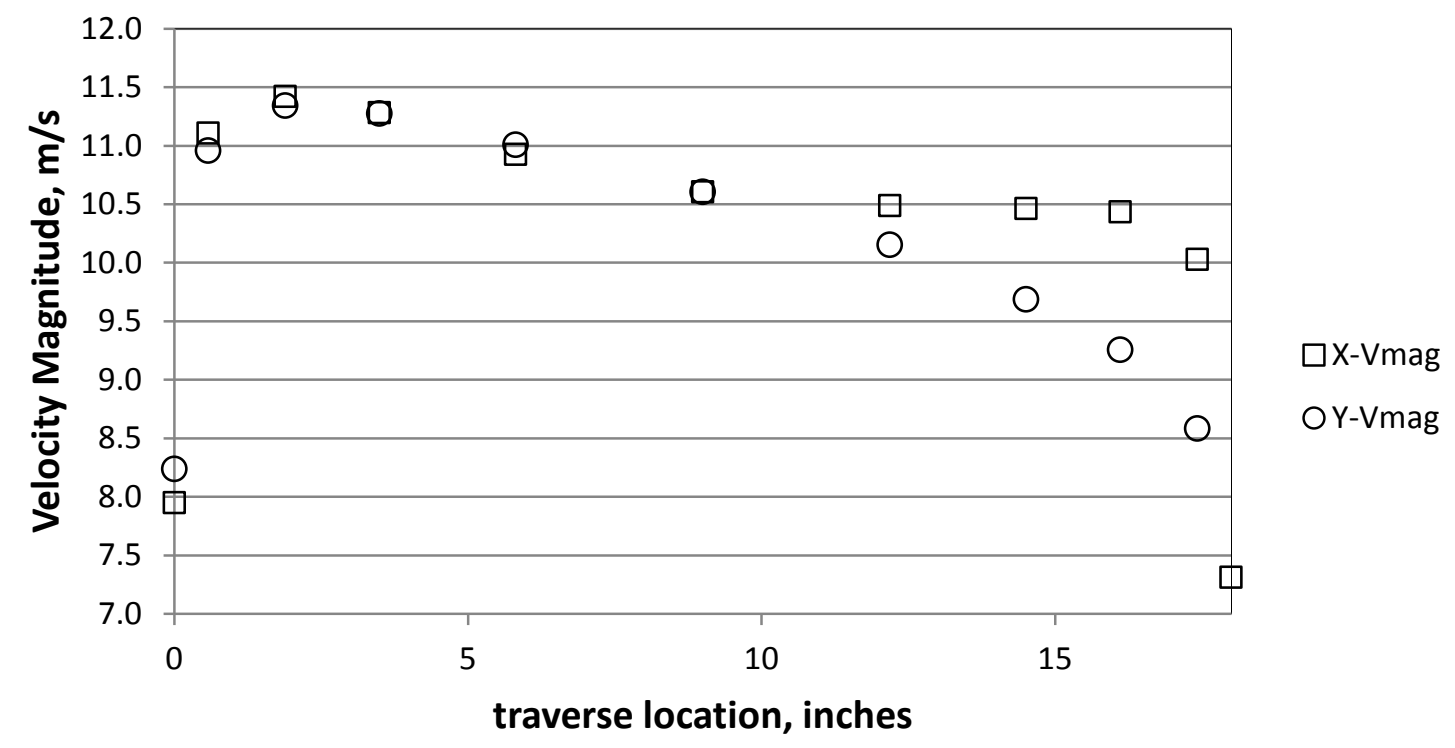

Figure H.21. Simulated results for the 2-fan configuration 2, 4: Flow velocity distribution at the elevation of the sampling system.

Flow Angle with 2-Fan Configuration 2, 4

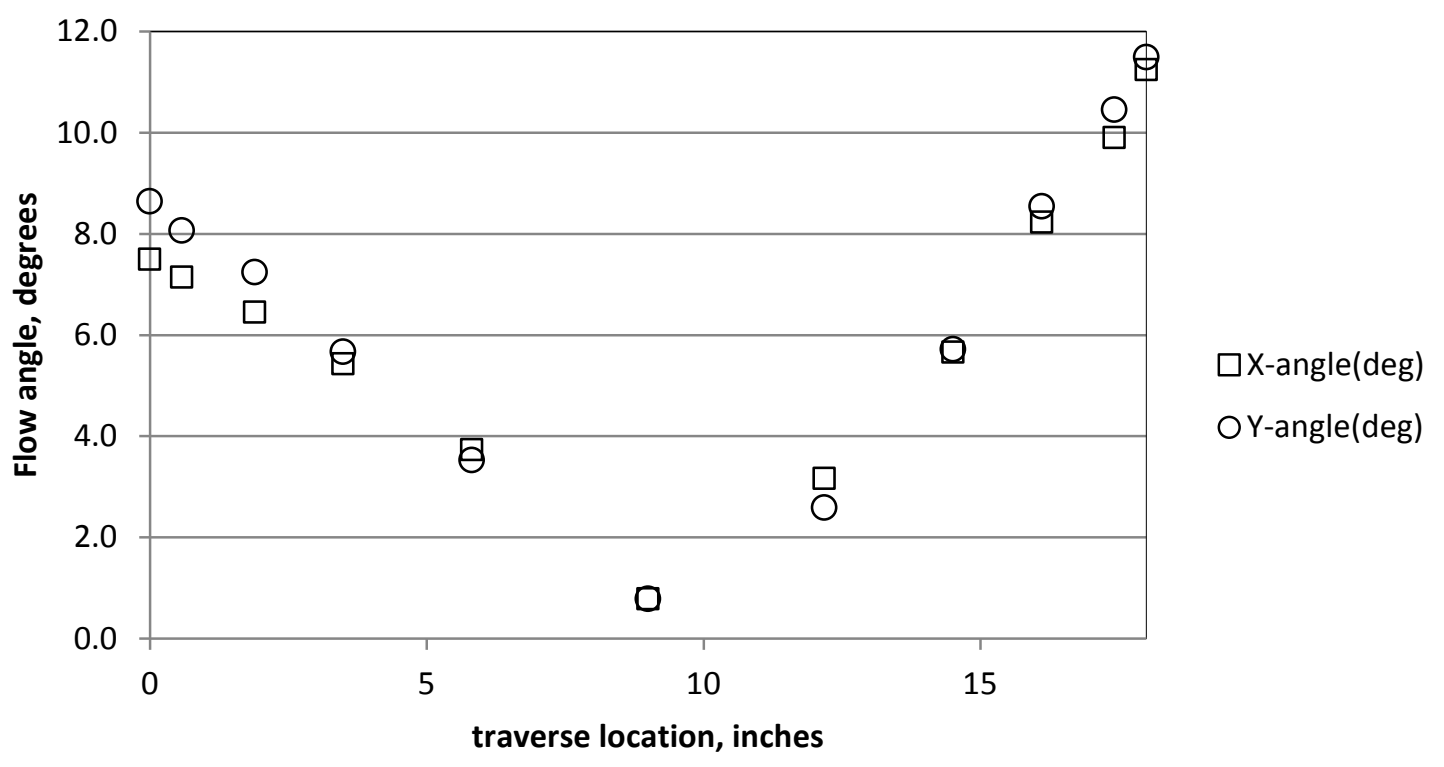

Figure H.22. Simulated results for the 2-fan configuration 2, 4: Cyclonic flow angle at the elevation of the sampling system. 


\section{Results: Operation of Fans 3 and 4}
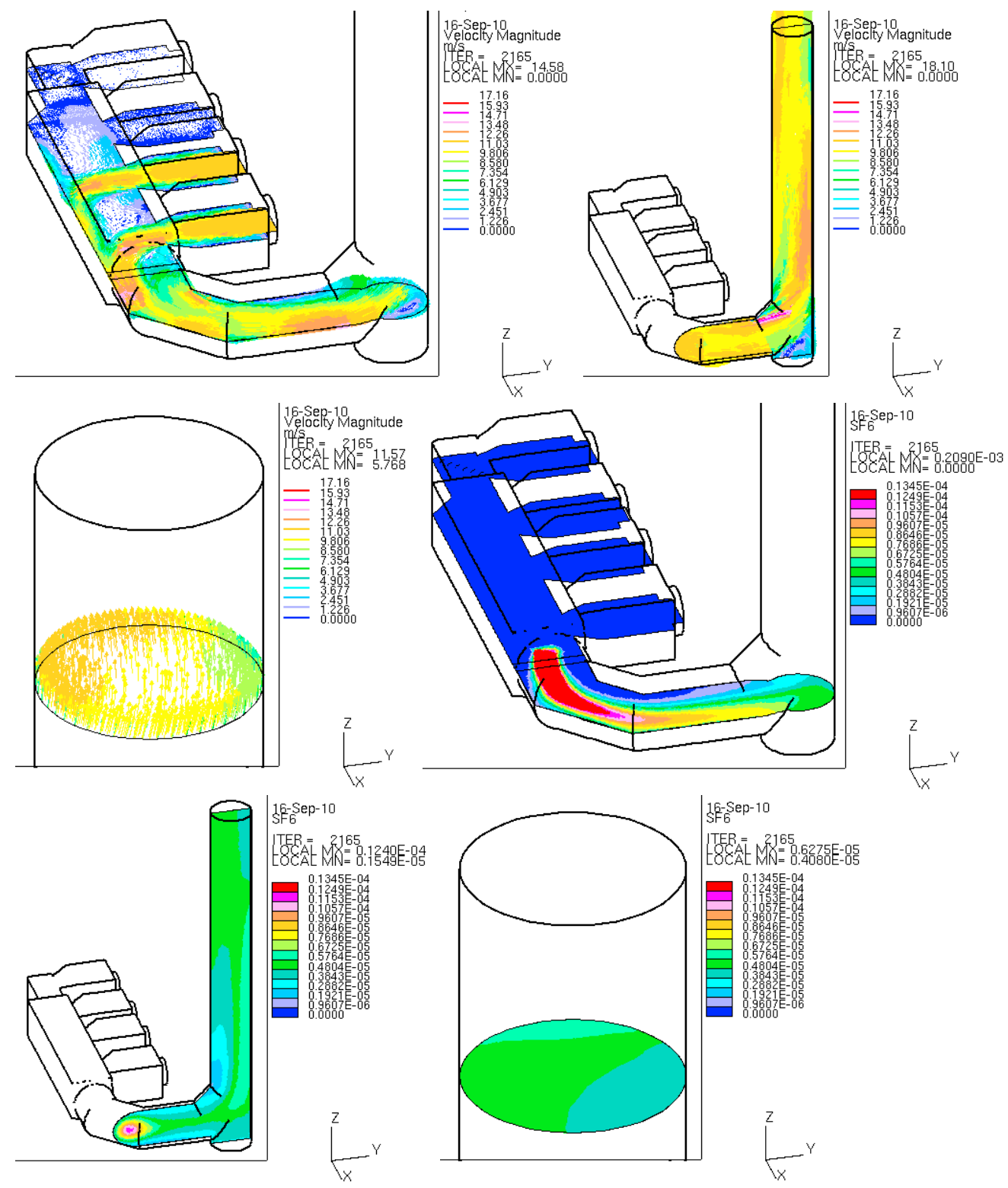

Figure H.23. Simulated flow velocity vectors and tracer gas concentrations for the case with fans 3 and 4 in operation: a) flow velocity in fans and lower ductwork, b) flow velocity in stack, c) velocity distribution in the stack at the elevation of the sampling system, d) tracer gas concentrations in the lower ductwork; tracer gas injected downstream of the fan nearest the stack, e) tracer gas concentrations in the stack, f) tracer gas distribution in the stack at the elevation of the sampling system. 


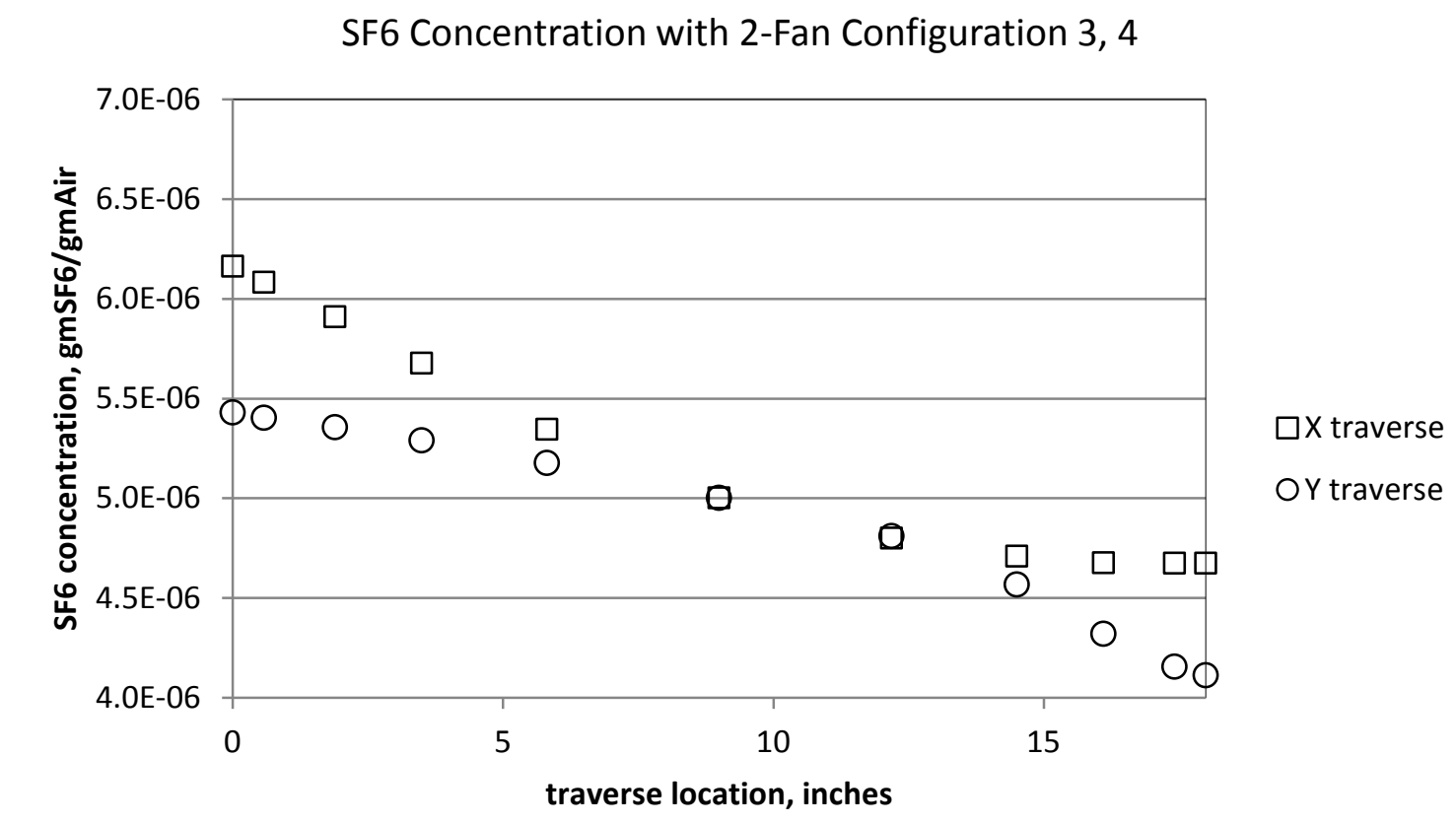

a)

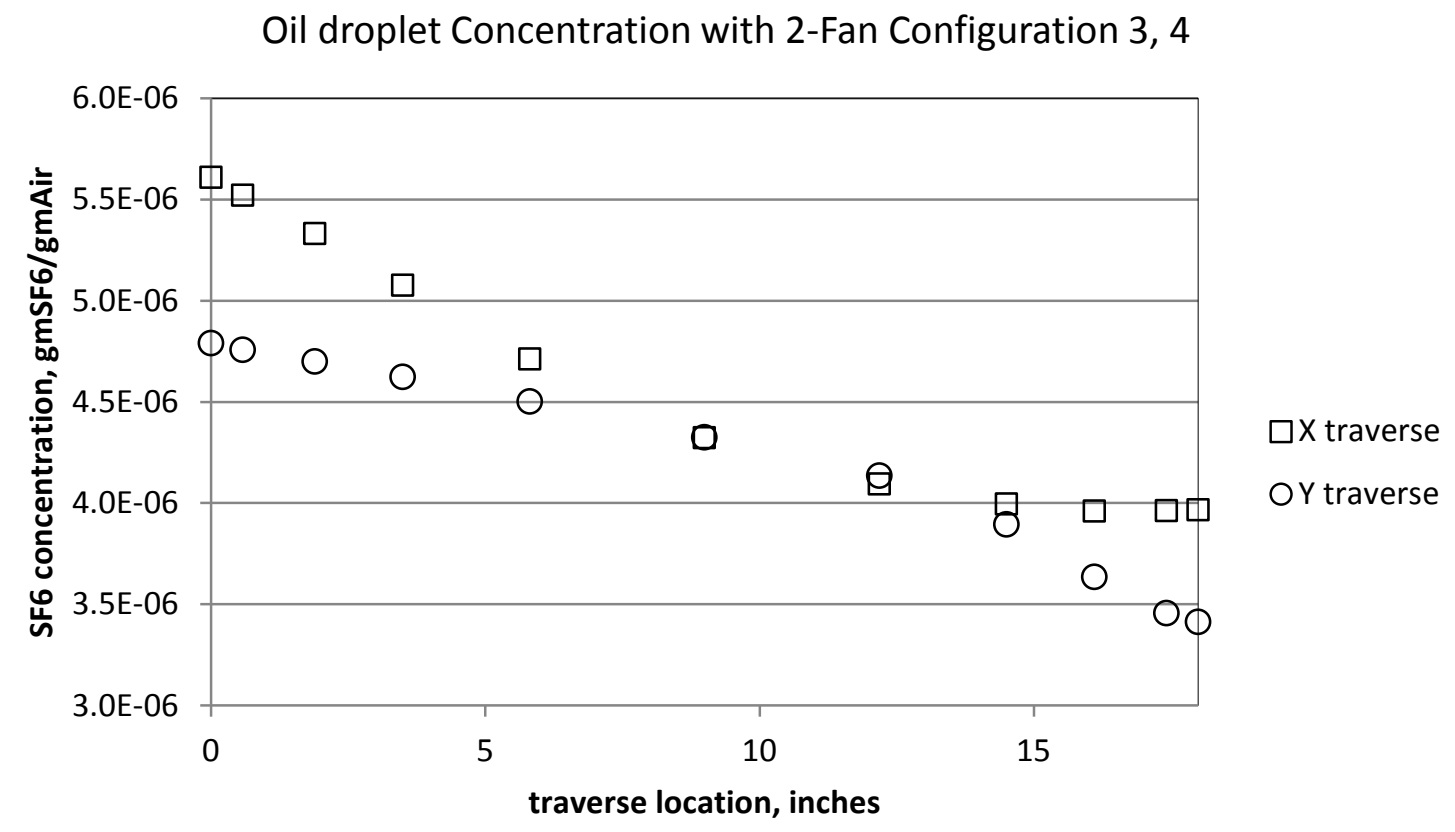

b)

Figure H.24. Simulated results for the 2-fan configuration 3, 4: Concentrations of a) SF6 tracer gas, and b) oil droplets at the elevation of the sampling system. 
Flow Velocity with 2-Fan Configuration 3, 4

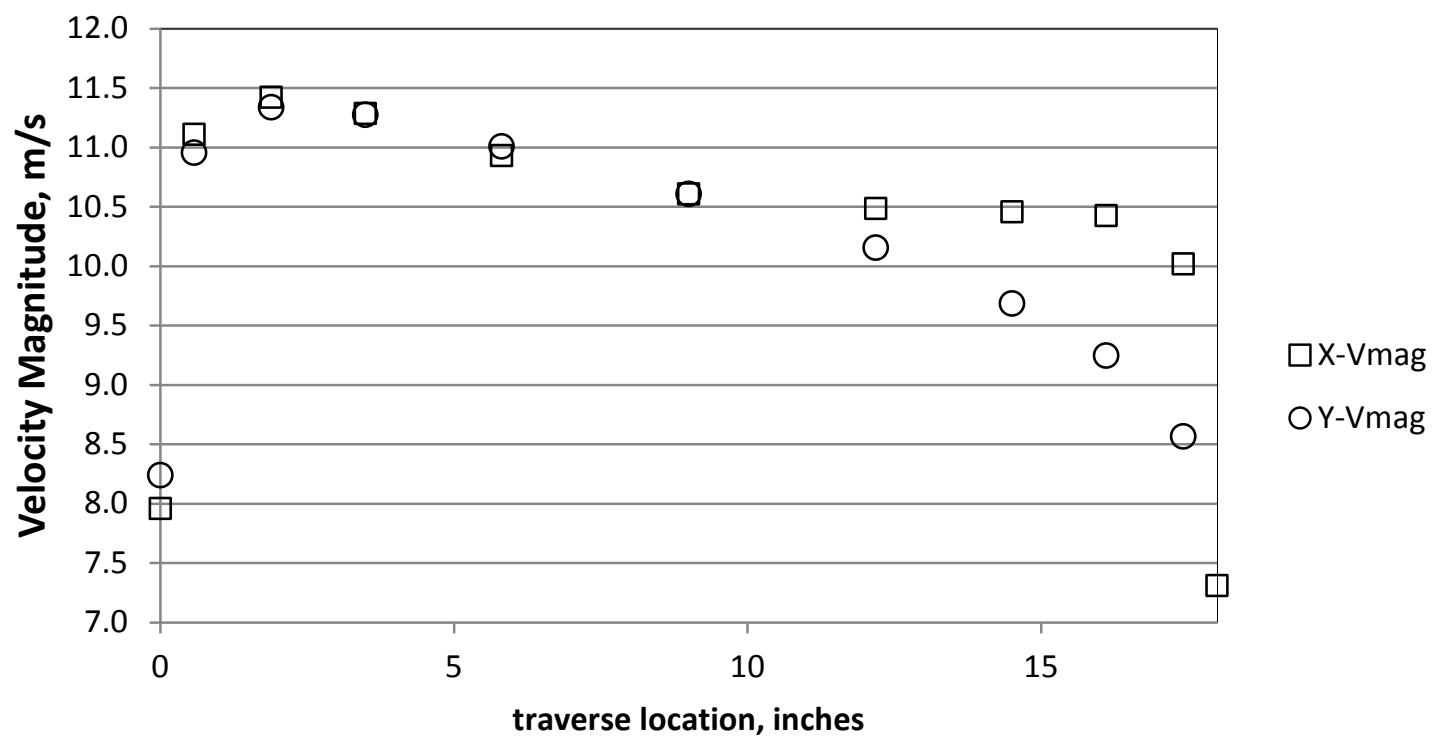

Figure H.25. Simulated results for the 2-fan configuration 3, 4: Flow velocity distribution at the elevation of the sampling system.

Flow Angle with 2-Fan Configuration 3, 4

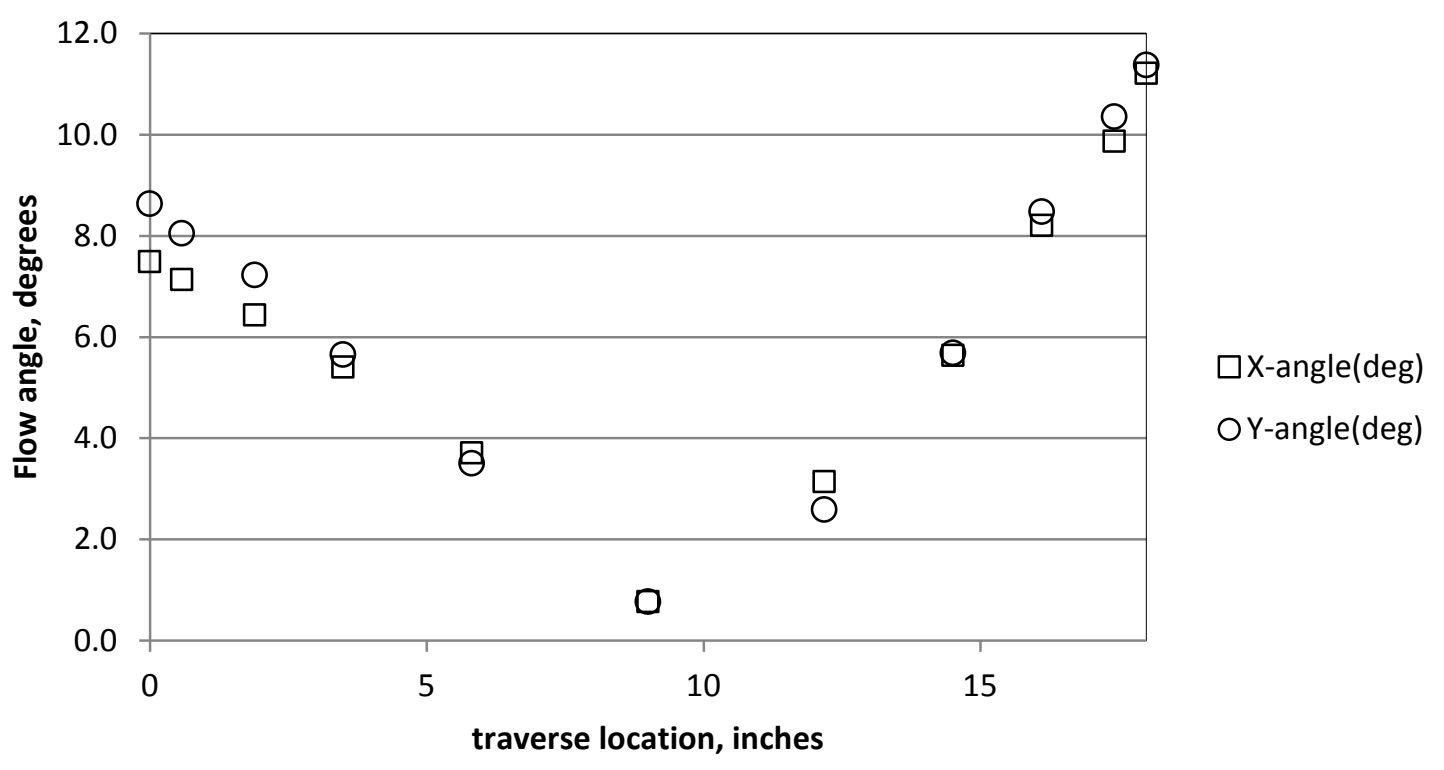

Figure H.26. Simulated results for the 2-fan configuration 3, 4: Cyclonic flow angle at the elevation of the sampling system. 



\section{Distribution}

No. of

Copies

(one [1] unless specified)

\begin{tabular}{|c|c|}
\hline \multicolumn{2}{|l|}{ Local Distribution } \\
\hline \multicolumn{2}{|c|}{ Pacific Northwest National Laboratory } \\
\hline MY Ballinger & BSRC \\
\hline JM Barnett & $\mathrm{J} 2-25$ \\
\hline SA Colby & P7-28 \\
\hline CJ Duchsherer & $\mathrm{J} 2-25$ \\
\hline JA Glissmeyer & K3-54 \\
\hline CA Kooiker & P7-28 \\
\hline KP Recknagle & K7-15 \\
\hline MJ Stephenson & $\mathrm{J} 2-25$ \\
\hline DG Wandler & P7-28 \\
\hline ST Yokuda & K7-15 \\
\hline EM File Plan T07.3.1.1.1 & $\mathrm{J} 2-25$ \\
\hline HTL & P8-5 \\
\hline
\end{tabular}

Distr.1 




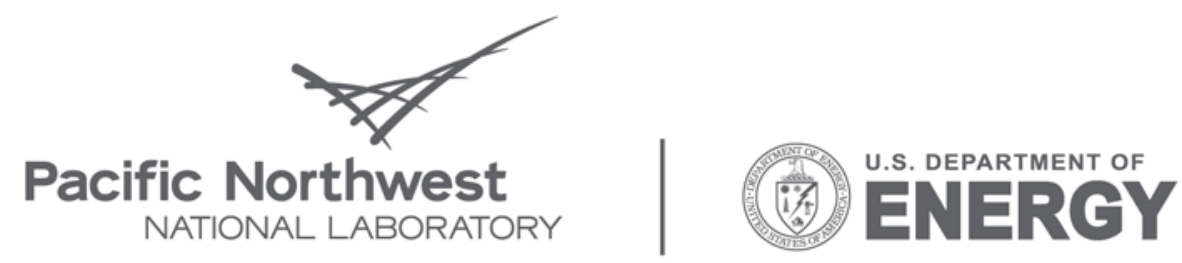

Proudly Operated by Battelle Since 1965

902 Battelle Boulevard

P.O. Box 999

Richland, WA 99352

1-888-375-PNNL (7665)

www.pnl.gov 